\title{
CZM-based Finite-Volume Homogenization and Optimization of Periodic Composites
}

\author{
A Dissertation \\ Presented to \\ the Faculty of the School of Engineering and Applied Science \\ University of Virginia
}

In Partial Fulfillment of the Requirements for the Degree of

Doctoral of Philosophy

(Civil Engineering/Applied Mechanics)

By

Wenqiong $\mathrm{Tu}$

August 2016 


\section{Approval Sheet}

This dissertation is submitted in partial fulfillment of the requirements for the degree of

Doctor of Philosophy (Civil Engineering/Applied Mechanics)

Wenqiong Tu

Author

This dissertation has been read and approved by the Examining Committee:

Marek-Jerzy Pindera (Advisor)

Thomas T. Baber (Chairman)

Houston G. Wood

Devin K. Harris

Osman E. Ozbulut

Richard W. Kent 
献给我亲爱的父母和弟弟:

涂端阳; 汪骄荣; 涂文琪

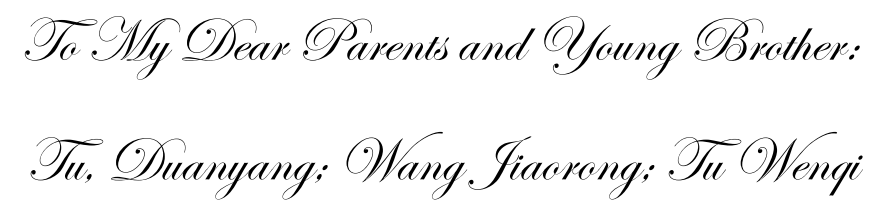




\section{Acknowledgements}

I would like to sincerely thank Professor Marek-Jerzy Pindera for his insightful guidance and unlimited support during my entire Ph.D. study. His kindness, patient and passion are deeply appreciated. The comments and suggestions from committee members: Professor Thomas T. Baber, Houston G. Wood, Devin K. Harris, Osman E. Ozbulut, Richard W. Kent, James G. Simmonds and Dr. Linfeng Chen during my Ph.D. qualify exam, proposal and final exam are gratefully appreciated. Thanks also go to my current colleague and former group members, Dr. Guannan Wang, Dr. Yabin Yang, Dr. Linfeng Chen, Dr. Marcio Cavalcante and Dr. Hamed Khatam.

I also want to thank Mollie, Professor Pindera's wife. Her kindness and consideration are greatly appreciated.

The author is very thankful for the funding support from the NSF Nano and BioMechanics Program Grant Award \#CMMI-1030673 and from the Department of Civil and Environmental Engineering through Graduate Teaching Assistantships and summer fellowships.

Special thanks go to the author's parents, Duanyang Tu, Jiaorong Wang and the Young brother, Wenqi Tu. Without their constant support and encouragement, the author would not be here.

Thank you! 


\section{Abstract}

A novel finite-volume based homogenization theory for damage evolution in periodic materials is proposed, verified and utilized to uncover previously undocumented phenomena at micro- and meso-levels. Displacement discontinuity functions are introduced into the formulation which facilitate separation of the system of equations that controls the response of a periodic composite undergoing damage evolution into primary and auxiliary systems, with the primary system solved just once. The formulation eliminates the necessity to re-assemble the global system of equations during damage evolution, determined by iteratively solving the auxiliary system of equations which governs damage progression. This formulation facilitates the implementation of any traction-displacement separation law to model damage, including traction-free boundary conditions to simulate crack growth and concomitant energy release rates, and a cohesive zone model (CZM) incorporated into the framework.

The proposed CZM-based finite-volume direct averaging micromechanics (FVDAM) theory is verified upon comparison with exact elasticity solutions in the elastic stage of damage evolution, and experimental data and finite-element simulations at both the micro-level and macro-level in the nonlinear stage. The comparison with finite-element simulations of interfacial debonding in a $\mathrm{SiC} / \mathrm{Titanium}$ composite reveals the beauty of the newly developed approach wherein once the stress normal to a failing interface become compressive, the corresponding governing equations are simply eliminated in the auxiliary system of equations to avoid interfacial interpenetration. This contrasts with the finite-element damage simulation approaches based on carefully chosen interfacial stiffness to resist the interpenetration. Following the extensive verification of CZM-based FVDAM, the newly developed approach is incorporated into a global optimization method, Particle Swarm Optimization (PSO) algorithm, and the marriage produces a powerful design tool for identifying optimal material architectures as well as parameters that are not easily measured experimentally, such as the CZM parameters or the elastic moduli of graphite fibers.

With the newly developed computation tool in modeling damage evolution in composite materials, three practical and important engineering problems are investigated and important fundamental findings are uncovered and documented. The first one is the study of interfacial debonding in $\mathrm{SiC} / \mathrm{Ti}$ unidirectional composites under transverse loading. The simulation 
produces good correlation with test data and correctly captures the evolution of fiber/matrix interfacial debonding and subsequent arrest due to the development of compressive stress normal to the interface. The importance of residual stresses is revealed, demonstrating that correct simulation of fiber/matrix debonding is not possible without these stresses.

The second application involves the simulation of evolving damage on the fly in polymeric matrix cross-ply laminates, caused by progressive cracking of the inner $90^{\circ}$ plies leading to subsequent delamination of adjacent plies. The effect of evolving damage on the homogenized axial stress-strain and transverse Poisson's responses, as well as crack density, are compared with available experimental results, taking account of residual stresses, interfacial resin-rich region and variable strength of the $90^{\circ}$ plies. The comparison demonstrates the theory's ability to capture the dramatic effect of transverse cracking on the homogenized transverse Poisson's ratio that increases with increasing $90^{\circ}$ ply thickness, and the damage mode bifurcation from transverse cracking to interfacial delamination, both for the first time. Moreover, the finite-volume simulations indicate that many features observed in the transverse and through-thickness Poisson's response of graphite/epoxy cross-ply laminates may be related to the underpinning damage modes more readily than in the axial response.

The last application involves simulation of damage in graphite/polyimide unidirectional off-axis specimens based on the hypothesis of shear-dominated fiber/matrix interfacial degradation as the primary cause of the observed nonlinearity. For the first time, this study reveals that the off-axis dependent nonlinearity in this material system comprised of elastic fibers and brittle, linearly elastic matrix may be accurately captured using a damage evolution model rather than plasticity, viscoelasticity or viscoplasticity approaches typically employed for the matrix phase.

The results presented in this dissertation demonstrate that the CZM-based FVDAM and the FVDAM-driven PSO algorithm are efficient and robust tools to model damage evolution in heterogeneous materials characterized by complex microstructures, optimize material performance through microstructural identification and calibrate micro-level material properties. 


\section{List of Figures}

Figure 1.1 Schematic figure of damage in a laminated plate under thermal shock (reproduced from the image on the webpage of Center for Nondestructive Evaluation, ISU).

Figure 1.2 Closure of an extended crack tip.

Figure 1.3 Eight-node: (a) parent element in $\eta-\xi$ plane, (b) quarter-point element in the $\mathrm{x}-\mathrm{y}$ plane and (c) Quarter-point collapsed triangular element.

Figure 1.4 Example of line J-integral path.

Figure 1.5 Discretization of unit cell and initial crack distribution with small crack.

Figure 1.6 Illustration of crack extension with different length.

Figure 1.7 Energy release rate for different crack lengths.

Figure 1.8 Control volumes employed in cell centered and vertex based finite-volume analyses of homogeneous material and structural problems in solid mechanics.

Figure 2.1 A reference square subvolume in the $\eta-\xi$ plane (left) mapped onto a quadrilateral subvolume in the $y_{2}-y_{3}$ plane (right) of the actual microstructure.

Figure 2.2 Interfacial discontinuity between two adjacent subvolumes.

Figure 2.3 Imposition of periodic boundary conditions for a square unit cell.

Figure 2.4 Traction-interfacial separation relations for the cohesive zone model in normal (left column) and tangential (right column) directions to the interface: graphical representations of (a) coupled and (b) uncoupled relations.

Figure 2.5 Unit cell geometry containing 0.05 fiber volume fraction (left) and a detailed close-up (right) used for comparison with the Eshelby solution for an inclusion with a linear interface in an infinite matrix.

Figure 2.6 Comparison of radial and tangential displacement discontinuities around the fiber/matrix interface obtained from dilute FVDAM and Eshelby solutions.

Figure 2.7 Comparison of normal and tangential stress fields in the region occupied by the unit cell obtained from dilute FVDAM and Eshelby solutions.

Figure 2.8 Comparison of normal and tangential stress fields in the region occupied by the unit cell obtained from dilute FVDAM and Eshelby solutions with the interfacial properties set to simulate the Kirsch solution. 
Figure 2.9 Comparison of displacement discontinuity and traction around the fiber/matrix interface obtained from dilute FVDAM and Eshelby solutions.

Figure 2.10 Comparison of out-of-plane shear stress fields in the region occupied by the unit cell obtained from dilute FVDAM and Eshelby solutions.

Figure 2.11 Comparison of out-of-plane shear stress fields in the region occupied by the unit cell obtained from dilute FVDAM and Eshelby solutions with extremely small interfacial properties.

Figure 3.1 Unit cell geometry and discretization containing 0.325 fiber volume fraction used in FVDAM (left) and Abaqus (right).

Figure 3.2 Schematics of 2D cohesive elements.

Figure 3.3 Node ordering and face numbering of cohesive element COH2D4 and positions of integration points and element centroid.

Figure 3.4 Linear damage evolution.

Figure 3.5 Schematic figure of square unit cell with fiber in center under plane strain condition.

Figure 3.6 Schematic figure of interfacial stiffness and compression factor, $f$.

Figure 3.7 Selected homogenized responses with different compression factors in $\mathrm{x}_{2}, \mathrm{x}_{3}$ directions under biaxial strain loading.

Figure 3.8 Interfacial displacement discontinuity (Left) and traction distributions (Right) around fiber/matrix interface at loading step 20.

Figure 3.9 Full-field stress distributions with different compression factors at loading step20.

Figure 3.10 Selected interfaces for the purpose of tracking interfacial separation process.

Figure 3.11 Homogenized response $\sigma_{22}$ and indication of loading steps at which the 1 st and 11 th interfaces separate completely.

Figure 3.12 Effective traction (a, d), normal traction (b, e), tangential traction (c, f) VS effective displacement discontinuity for 1st interface (left) and 11th interface (right) under biaxial strain loading.

Figure 3.13 Homogenized response $\sigma_{23}$ and indication of loading steps at which the 1 st and 11th interfaces separate completely. 
Figure 3.14 Effective traction (a, d), normal traction (b, e), tangential traction (c, f) VS effective displacement discontinuity for 1st interface (left) and 11th interface (right) under transverse shear loading.

Figure 3.15 Selected homogenized responses in FVDAM and Abaqus under biaxial strain loading.

Figure 3.16 Interfacial traction and displacement discontinuity distributions in FVDAM and Abaqus at step 1.

Figure 3.17 Interfacial traction and displacement discontinuity distributions in FVDAM and Abaqus at step 10.

Figure 3.18 Interfacial traction and displacement discontinuity distributions in FVDAM and Abaqus at step 30 .

Figure $3.19 \sigma_{22}$ distributions in FVDAM and Abaqus at difference steps.

Figure 3.20 $\sigma_{33}$ distributions in FVDAM and Abaqus at difference steps.

Figure 3.21 $\sigma_{23}$ distributions in FVDAM and Abaqus at difference steps.

Figure 3.22 Selected homogenized responses with FVDAM and Abaqus under transverse shear.

Figure 3.23 Interfacial traction and displacement discontinuity distributions in FVDAM and Abaqus at step 1 .

Figure 3.24 Interfacial traction and displacement discontinuity distributions in FVDAM and Abaqus at step 20.

Figure 3.25 Interfacial traction and displacement discontinuity distributions in FVDAM and Abaqus at step 50.

Figure $3.26 \sigma_{22}$ distributions in FVDAM and Abaqus at difference steps.

Figure 3.27 $\sigma_{33}$ distributions in FVDAM and Abaqus at difference steps.

Figure $3.28 \sigma_{23}$ distributions in FVDAM and Abaqus at difference steps.

Figure 3.29 Homogenized response predicted by FVDAM and Abaqus when compression factor of 100 is used in Abaqus simulations.

Figure 3.30 Homogenized response of FVDAM and Abaqus when compression factor of 10 is used in Abaqus simulations.

Figure 4.1 Unit cell geometry and discretization containing 0.325 fiber volume fraction. 
Figure 4.2 Initial transverse response of the unidirectional SiC/Ti composite with different interfacial strengths, illustrating the effect of fabrication-induced residual stresses.

Figure 4.3 Comparison of the applied (vertical axis) and calculated (horizontal axis) homogenized strains based on Eq. (2.33), demonstrating consistency and accuracy of the implemented solution technique for the nonlinear response of the unit cell based on the implemented CZM, and importance of the contributions of the interfacial displacement discontinuities toward total homogenized strains.

Figure 4.4 Initial transverse response of the unidirectional SiC/Ti composite immediately after fabrication cooldown, illustrating the effect of (a) uncoupled and (b) coupled interfacial separation laws.

Figure 4.5 Interfacial displacement discontinuity and traction distributions around the fiber/matrix interface with progressively greater applied load after fabrication cooldown.

Figure 4.6 Full-field stress distributions at progressively greater applied load after fabrication cooldown.

Figure 4.7 Transverse response of the representative unit cell of unidirectional SiC/Ti composite with different interfacial debonding lengths after initial preloading.

Figure 4.8 Interfacial displacement discontinuity and traction distributions around the fiber/matrix interface with progressively greater applied load after initial preloading.

Figure 4.9 Full-field stress distributions at progressively greater applied load after fiber/matrix degradation by initial preloading.

Figure 4.10 Unit cell geometry and discretization containing 0.325 fiber volume fraction.

Figure 4.11 Initial transverse response generated via square and hexagonal unit cells for unidirectional $\mathrm{SiC} / \mathrm{Ti}$ composite immediately after fabrication cooldown with uncoupled separation laws.

Figure 4.12 Interfacial displacement discontinuity and traction distributions around the fiber/matrix interface under $-555^{\circ} \mathrm{C}$ cool-down.

Figure 4.13 Interfacial displacement discontinuity and traction distributions around the fiber/matrix interface at step 5. 
Figure 4.14 Interfacial displacement discontinuity and traction distributions around the fiber/matrix interface at step 13.

Figure 4.15 Interfacial displacement discontinuity and traction distributions around the fiber/matrix interface at step 25 .

Figure 4.16 Full-field stress distributions after $555^{\circ} \mathrm{C}$ fabrication cool-down.

Figure 4.17 Full-field stress distributions at step 13.

Figure 4.18 Initial transverse and through-thickness response generated via square and hexagonal unit cells for unidirectional $\mathrm{SiC} / \mathrm{Ti}$ composite immediately after fabrication cooldown with uncoupled separation laws.

Figure 4.19 Interfacial displacement discontinuity and traction distributions around the fiber/matrix interface at step 5 under transverse and through-thickness loadings.

Figure 4.20 Interfacial displacement discontinuity and traction distributions around the fiber/matrix interface at step 13 under transverse and through-thickness loading.

Figure 4.21 Full-field stress distributions at step 5 along transverse loading (left) and along through-thickness loading (right).

Figure 4.22 Full-field stress distributions at step 13 along transverse loading (left) and along through-thickness loading (right).

Figure 5.1 Flow chart showing the marriage of FVDAM and PSO algorithm.

Figure 5.2 Longitudinal stress-strain response of porcine mitral valve marginal, basal and strut chordae (reproduced by digitization from the experimental data of Liao and Vesely, 2003b).

Figure 5.3 Geometry of the simplified model of the microstructure of porcine mitral valve strut chordae employed in the FVDAM simulations based on the reported mean values of amplitude and crimp period (Liao and Vesely, 2003a). The top representative unit cell is the reference cell with thickest stiff layer which is further subdividing in a manner that retains its volume fraction (middle and bottom unit cells). Detail of the unit cell discretization is shown in the bottom unit cell (note: axes scale is in microns).

Figure 5.4 The error convergence with iteration number of the PSO algorithm in simulating the homogenized response of porcine mitral valve marginal, basal and strut chordae. 
Figure $5.5 \quad$ (a) Initial and final particle distributions. (b) Best initial and final simulated homogenized responses of the marginal chordae tendineae.

Figure 5.6 (a) Initial and final particle distributions. (b) Best initial and final simulated homogenized responses of the basal chordae tendineae.

Figure 5.7 (a) Initial and final particle distributions. (b) Best initial and final simulated homogenized responses of the strut chordae tendineae.

Figure 5.8 (a) Optimization convergence curve; (b) Comparison between experimental data and optimized homogenized response.

Figure 5.9 (a) Optimization convergence curve; (b) Initial particle distribution and final particle distribution.

Figure 5.10 Comparison between experimental data and optimized homogenized response.

Figure 5.11 Active fiber/matrix interface distribution in a hexagonal unit cell: (a) 100\% active interface; (b) $75 \%$ active interface; (c) $50 \%$ active interface.

Figure 5.12 Convergence responses for three types of interface distributions as shown in Figure 5.11.

Figure 5.13 Particles' distributions for case (a), (b), (c).

Figure 5.14 Optimized homogenized responses for three types of interface distributions.

Figure 5.15 Full-field stress distributions at step 10 for case (a), (b) and (c).

Figure 5.16 Full-field stress distributions at step 20 for cases (a), (b) and (c).

Figure 6.1 (a) Hexagonal unit cell of unidirectional AS4/3501 graphite/epoxy with fiber volume fraction of 0.63 used to generate the missing thermo-elastic moduli for laminate analysis; (b) unit cell of a $\left[0 \frac{0}{2} / 90_{2}^{0}\right]_{\mathrm{S}}$ laminate.

Figure 6.2 Stress distributions in $90^{\circ}$ and $0^{\circ}$ plies of a graphite/epoxy $\left[02 / 90_{2}^{0}\right]_{\mathrm{s}}$ laminate in the vicinity of an isolated vertical crack (crack density of 0.19 cracks $/ \mathrm{mm}$ ) at different elevations from the midplane. Comparison with the variational solution of Vasilev et al. (1970).

Figure 6.3 Stress distributions in $90^{\circ}$ and $0^{\circ}$ plies of a graphite/epoxy $\left[00_{2}^{0} / 90_{2}^{0}\right]_{\mathrm{s}}$ laminate between interacting vertical cracks (crack density of 0.95 cracks $/ \mathrm{mm}$ ) at different elevations from the midplane. Comparison with the variational solution of Vasilev et al. (1970). 
Figure 6.4 Effect of resin-rich layer and crack density on stress distributions between adjacent vertical cracks in $90^{\circ}$ plies of a $\left[0 \frac{2}{9} / 90_{2}^{0}\right]_{\mathrm{s}}$ laminate: (a) throughthickness $\sigma_{x x}$ stress distributions halfway between vertical cracks; (b-c) $\sigma_{x z}$ and $\sigma_{z z}$ stress distributions along the $0^{\circ} / 90^{\circ}$ interface.

Figure 6.5 Effect of interfacial debonding between the resin-rich layer at the $0^{\circ} / 90^{\circ}$ interface and the $0^{\circ}$ ply on stress distributions between adjacent vertical cracks in $90^{\circ}$ plies of a $\left[0 \frac{0}{2} / 90_{2}^{0}\right]_{\mathrm{s}}$ laminate: (a) through-thickness $\sigma_{x x}$ stress distributions halfway between vertical cracks; (b-c) $\sigma_{x z}$ and $\sigma_{z z}$ stress distributions along the $0^{\circ} / 90^{\circ}$ interface.

Figure 6.6 Effect of fabrication cooldown on the microlevel $\sigma_{\mathrm{xx}}$ stress fields in stand-alone (left) and in-situ (right) $90^{\circ}$ plies at failure.

Figure 6.7 Homogenized axial, transverse and through-thickness Poisson's responses of a $\left[0_{2}^{0} / 90_{2}^{0}\right]_{\mathrm{s}}$ laminate under uniaxial loading, and crack evolution with applied stress, demonstrating the effects of resin-rich interfacial layer and transverse interface strength variability of the $90^{\circ}$ plies.

Figure 6.8 Through-thickness axial stress distributions along vertical interfaces that have not failed up to Step 51, illustrating gradual transverse crack progression with increasing load that does not produce a discontinuity in the transverse Poisson's response (see Figure 6.7a).

Figure 6.9 (a) Initiation and progression of delamination along the $0 \% / 90^{\circ}$ interface of a $\left[0_{2}^{0} / 90_{2}^{0}\right]_{\mathrm{s}}$ laminate in the vicinity of a vertical crack in the $90^{\circ}$ plies; (b) corresponding stress fields.

Figure 6.10 Homogenized transverse Poisson's response of a $\left[0 \frac{0}{2} / 90_{2}^{0}\right]_{\mathrm{s}}$ laminate under uniaxial loading, and crack evolution with applied stress, demonstrating the effects of horizontal interface strength variability of the $90^{\circ}$ plies.

Figure 6.11 Crack density evolution as a function of applied axial stress in cross-ply laninates with different thickness of the inner $90^{\circ}$ plies tested by Wang et al. (1985) under uniaxial loading.

Figure 6.12 Predicted homogenized transverse Poisson's responses of cross-ply laminates with different thickness of the inner $90^{\circ}$ plies tested by Wang et al. (1985) uniaxial loading. 
Figure 7.1 Pure tensile response along the fiber direction (a) and perpendicular to the fiber direction (b), and shear response in the principal material coordinate system of a $15^{0}$ off-axis specimen subjected to uniaxial loading in the laminate coordinate system (c).

Figure 7.2 An off-axis specimen uniaxially loaded in the global coordinate and the corresponding transformed combined in-plane stresses in the principal material coordinate system.

Figure 7.3 Hexagonal unit cell with volume fraction of $61 \%$, which is the average value of the recorded volume fraction.

Figure 7.4 Convergence curve.

Figure 7.5 Interface distributions inside a hexagonal unit cell.

Figure 7.6 Homogenized responses with interfacial strength 61MPa and different final separation distance.

Figure 7.7 Stress distributions at damage initiation step and final loading step.

Figure 7.8 Homogenized response under transverse loading with and without consideration of temperature cool-down.

Figure 7.9 Full-field stress distributions after temperature cool-down of $302^{\circ} \mathrm{C}$.

Figure 7.10 Full-field stress distributions at step 24 with and without temperature cool-down.

Figure 7.11 Full-field stress distributions at step 34 with and without temperature cool-down.

Figure 7.12 Interface distributions inside a hexagonal unit cell.

Figure 7.13 Homogenized shear response of $15^{\circ} \mathrm{C}$ off-axis specimen for different interface distributions.

Figure 7.14 Stress distributions for two types of interface configuration at step 10.

Figure 7.15 Stress distributions for two types of interface configuration at step 14.

Figure 7.16 Stress distributions for two types of interface configuration at step 18.

Figure 7.17 Homogenized shear responses under $10^{\circ}$ (Left) and $45^{\circ}$ (Right) off-axis loading.

Figure 7.18 Shear responses under different off-axis loading angles and FVDAM's prediction.

Figure 7.19 Comparison between experimental data and FVDAM's predictions for laminate response under different off-axis loading axis. 


\section{List of Tables}

Table 2.1 Elastic parameters for the modified Eshelby problem.

Table 3.1 Elastic properties of the SiC fibers and Ti matrix.

Table 3.2 Interfacial strength parameters for the SiC/Ti composite. Note: $\mathrm{k}_{\mathrm{n}}^{0}=\sigma_{\max } / \Delta_{\mathrm{n}}^{\mathrm{o}}$.

Table 4.1 Thermoelastic properties of the SiC fibers and Ti matrix.

Table 4.2 Interfacial strength parameters for the SiC/Ti composite. Note: $\mathrm{k}_{\mathrm{n}}^{0}=\sigma_{\max } / \Delta_{\mathrm{n}}^{\mathrm{o}}$.

Table 5.1 Parameters used in the Particle Swarm Optimization algorithm.

Table 5.2 Ranges of the amplitude and wavelength of the crimped microstructure of the porcine mitral valve marginal, basal and strut chordae tendineae and the Young's modulus of the limiting linear response (Liao and Vesely, 2003a) employed in the construction of unit cell models and the extraction of hyperelastic constitutive model parameters.

Table 5.3 Elastic parameters of the generalized Mooney-Rivlin model extracted from the experimental data on the porcine mitral valve marginal, basal and strut chordae tendineae (Liao and Vesely, 2003a) employed in the simplified unit cell models for the response of chordae tendineae constituents. The marginal, basal and strut layers are the stiff layers in the simplified model and the matrix is the soft layer.

Table 5.4 Optimized design variables for three types of interface distributions.

Table 6.1 Thermoelastic properties of the AS4 graphite fiber and 3501 epoxy matrix. Note that the AS4 fiber is assumed to be transversely isotropic with $\mathrm{E}_{22}=\mathrm{E}_{33}, v_{12}=$ $v_{13}$ and $\mathrm{G}_{23}=\mathrm{E}_{22} / 2\left(1+v_{23}\right)$ and the epoxy matrix is isotropic (Soden et al. 1998).

Table 6.2 Thermoelastic properties of the unidirectional AS4/3501 graphite/epoxy laminate with the fiber volume fraction of 0.63 , measured experimentally in the case of the moduli $E_{11}, E_{22}$ and $G_{12}$ and $v_{12}$ and in the case of $G_{23}, \alpha_{11}$ and $\alpha_{22}$ calculated using the homogenization FVDAM theory.

Table 6.3 Thermoelastic properties of the unidirectional graphite/epoxy laminate tested by Wang et al. (1985).

Table 6.4 Cohesive zone model parameters for the graphite-epoxy $\left[0_{2}^{0} / 90_{2}^{0}\right]_{\mathrm{s}}$ laminate tested by (Pindera, 1986). Note: $\mathrm{k}_{\mathrm{n}}^{0}=\sigma_{\max } / \Delta_{\mathrm{n}}^{0}$. The parameters for the $0^{0} / 90^{0}$ 
interface are based on the strain energy release rates of $G_{I C}=298 \mathrm{~J} / \mathrm{m}^{2}$ and $\mathrm{G}_{\mathrm{IIC}}=142 \mathrm{~J} / \mathrm{m}^{2}$, (Wilkins et al. 1982).

Table 7.1 Ply properties of Celion 6000/PMR-15 (Pindera, 1981).

Table 7.2 PMR-15 matrix properties (Pindera, 1981) and the thermal expansion coefficients (Papadopoulos and Bowles, 1990).

Table 7.3 T300 fiber properties (Pindera 1981) and thermal expansion coefficients (Papadopoulos and Bowles, 1990).

Table 7.4 Design variables range for Celion 6000 fiber properties (20\%).

Table 7.5 Optimized design variables.

Table 7.6 Test data and homogenized properties based on optimized material properties.

Table 7.7 Interfacial parameters used for transverse loading.

Table 7.8 Interfacial parameters for out-of-plane shear response. 


\section{Contents}

1 Introduction....................................................................................................................................................... 1

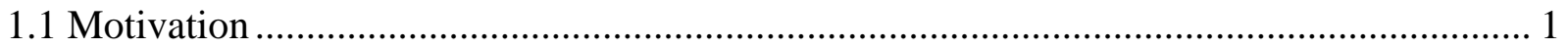

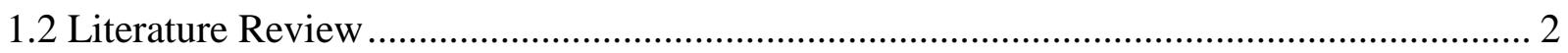

1.2.1 Stress Intensity Factor and Energy Release Rate Calculations .................................... 2

1.2.1.1 Analytical Approach ............................................................................................. 4

1.2.1.2 Numerical Approach................................................................................... 7

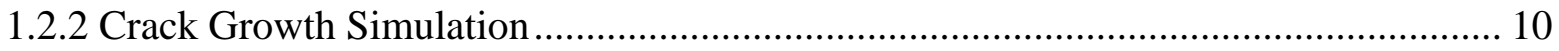

1.2.3 Crack Initiation and Propagation Simulation ........................................................... 14

1.3 Finite-Volume Theories in Solid Mechanics Applications ............................................... 15

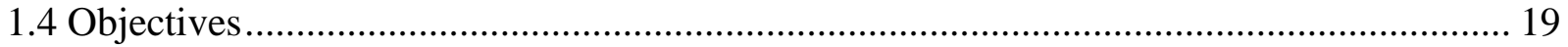

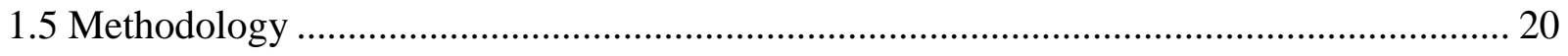

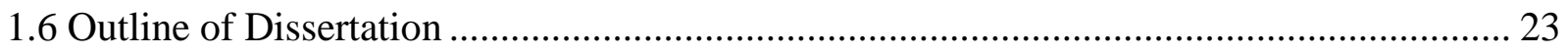

2 Cohesive Zone Model-Based Finite-Volume Homogenization Theory .................................. 24

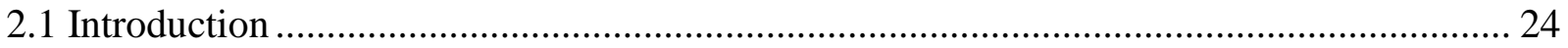

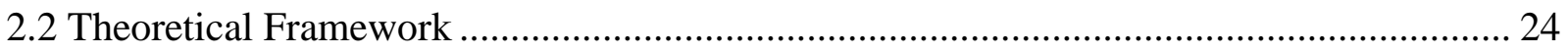

2.3. Traction and Displacement Continuity at Subvolume Interfaces...................................... 29

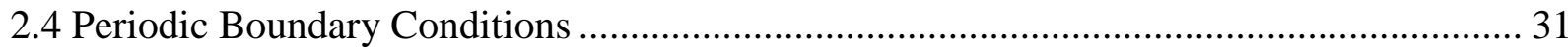

2.5 Primary and Auxiliary Global Systems of Equations …………………........................... 32

2.6 Cohesive Zone Model Implementation ........................................................................... 33

2.7 Homogenization ........................................................................................................ 37

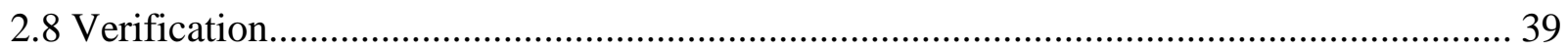

2.8.1 In-Plane Far-Field Loading ..................................................................................... 39

2.8.2 Out-of-Plane Far-Field Loading ………………..................................................... 44

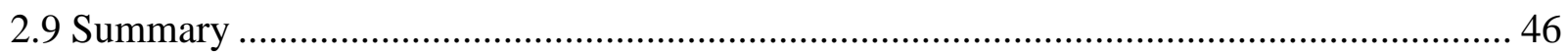

3 Assessment of CZM-FVDAM and Abaqus Predictive Capabilities .......................................... 48

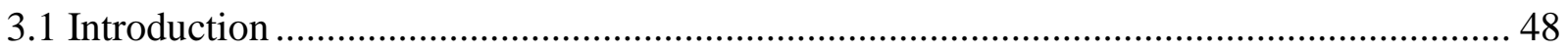

3.2 Model Set-Up in CZM-FVDAM and Abaqus.................................................................. 49

3.2.1 Unit Cell Geometry and Discretization ......................................................................... 49

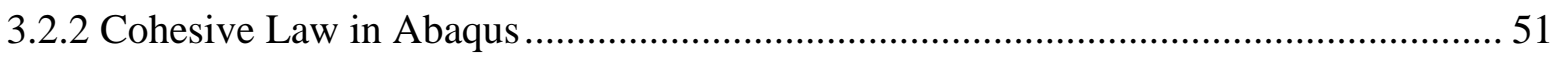


3.2.3 Imposition of Periodic Boundary Conditions in Abaqus........................................ 52

3.3 Effect of Interfacial Stiffness under Compressive Normal Stress.................................. 53

3.4 Damage Evolution along Specific Interfaces ........................................................ 57

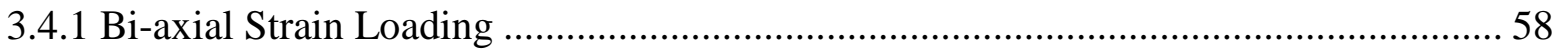

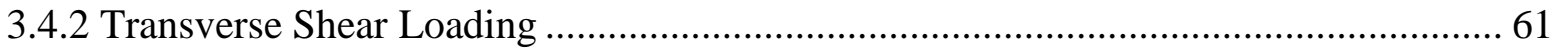

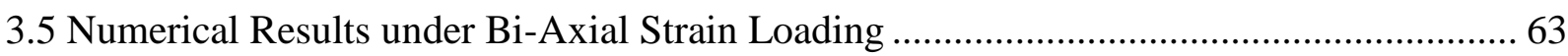

3.6 Numerical Results under Transverse Shear .............................................................. 70

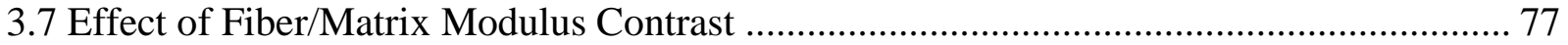

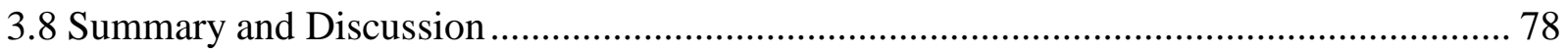

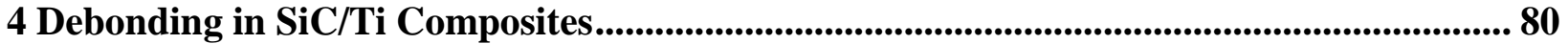

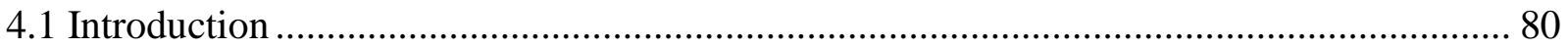

4.2 Progressive Interfacial Degradation after Fabrication Cooldown.................................. 82

4.3 Progressive Interfacial Degradation after Initial Preload Cycles .................................. 88

4.4 Effect of Unit Cell Architecture on Interfacial Damage ................................................ 92

4.5 Effect of Loading Direction for Hexagonal Unit Cell.............................................. 100

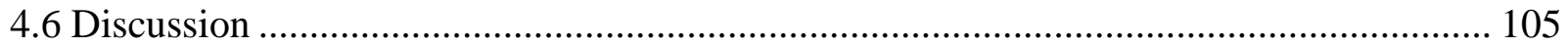

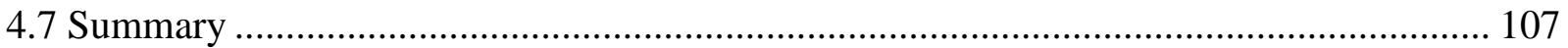

5 Finite Volume Micromechanics-Driven Particle Swarm Optimization............................ 108

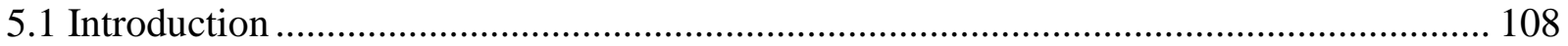

5.2 FVDAM-Driven Particle Swarm Optimization ....................................................... 109

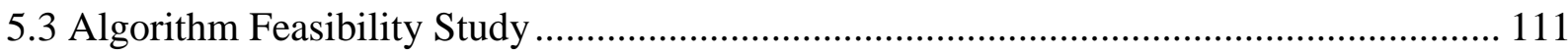

5.3.1 Model of the Mitral Valve Chordae Tendineae ................................................... 111

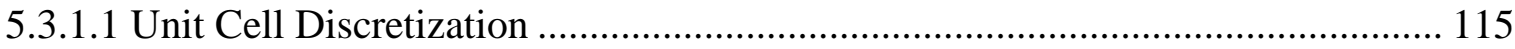

5.3.1.2 Phase Constitutive Response ................................................................. 115

5.3.2 Targeting the Response Using Particle Swarm Optimization ................................ 117

5.4 Optimizing CZM Parameters for Interfaces Undergoing Damage ................................ 120

5.4.1 Interfacial Parameter Optimization based on Square Unit Cell ............................. 120

5.4.2 Effect of Randomness of Active Interfaces on Optimization................................. 123

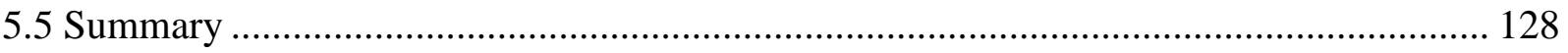

6 Damage Evolution in Cross-Ply Laminates Revisited ......................................................... 130

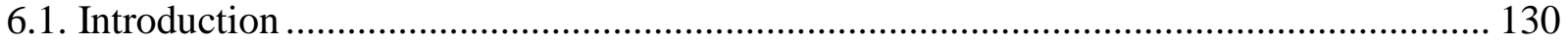




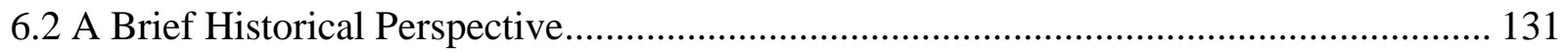

6.3 Material Systems Used in Numerical-Experimental Correlation ..................................... 133

6.4 Damage Modes and Enabling Stress Fields ................................................................ 135

6.5 Homogenized Axial and Transverse Response: Theory vs Experiment ........................... 142

6.5.1 The Effect of Residual Stresses ............................................................................ 143

6.5.2 Features of the Homogenized Response and Underpinning Mechanisms ................. 145

6.5.3 Damage Mode Bifurcation ..................................................................................... 149

6.6 Crack Density Evolution - Theory vs Experiment ………………………………….... 152

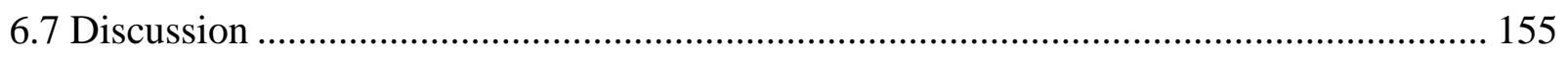

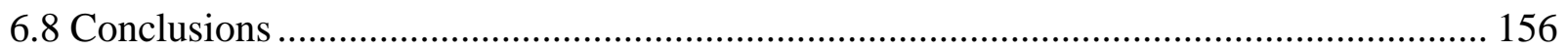

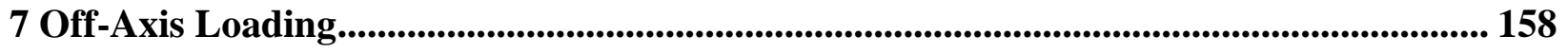

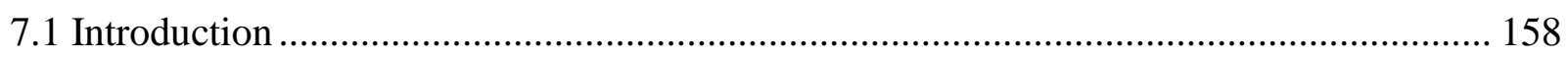

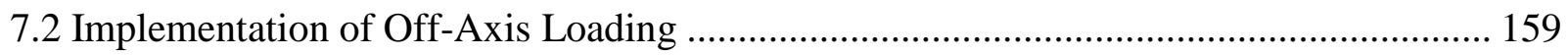

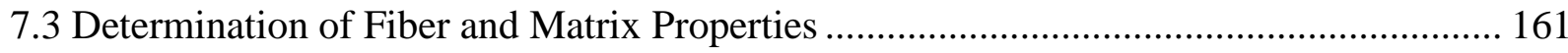

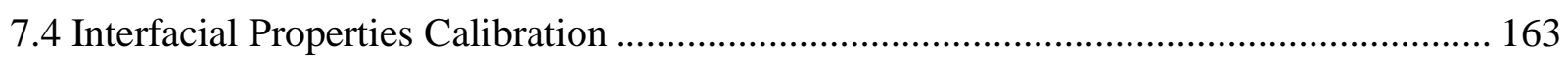

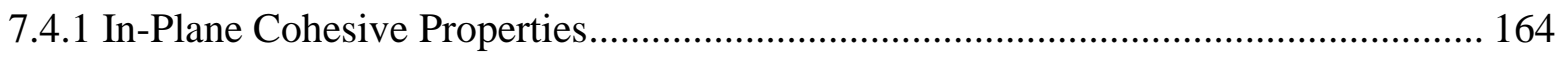

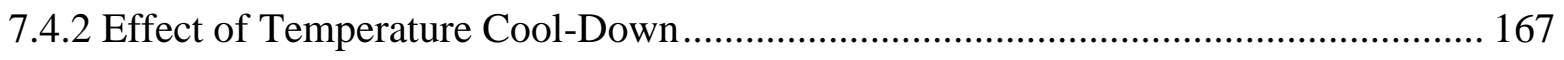

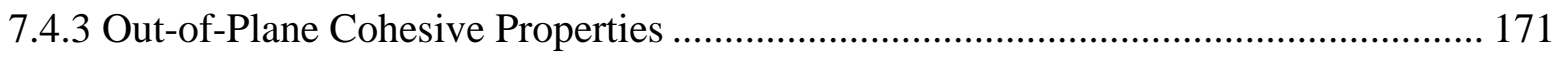

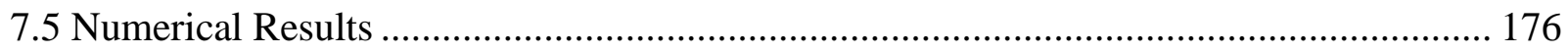

7.5.1 Monotonic Response in Principal Material Coordinates ............................................... 176

7.5.2 Monotonic Response in the Laminate Coordinate System ......................................... 177

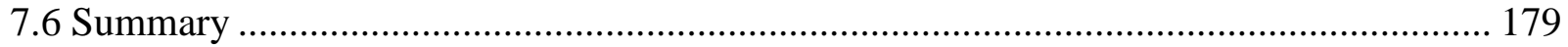

Accomplishments, Summary and Conclusions ................................................................................. 180

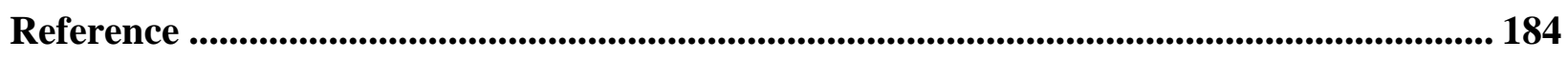

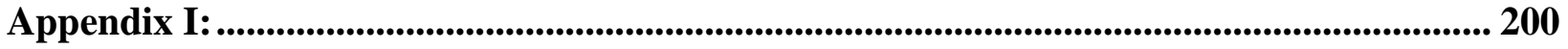




\section{Chapter1}

\section{Introduction}

\subsection{Motivation}

Fiber-reinforced composites are used with increasing frequency in aerospace, civil, marine, automotive and many other industries due to their high performance. To make good use of these materials, it is critically important to have a good understanding and predictive capability of their failure behavior. Simulation of damage evolution in fiber-reinforced materials remains a challenging problem due to the myriad of failure mechanisms and modes, and their complex nature that may be activated at different scales. As illustrate in Figure 1.1, in composite laminates comprised of differently-oriented unidirectional plies, for example, these modes, which are hierarchical and may interact with each other, include microcracks, fiber/matrix debonding, ply cracking and interfacial delaminations, (Hassan and Batra 2008, Njuhovic et al. 2015). For instance, accumulation of microcracks in the matrix phase can cause ply cracking, and ply cracking may induce delamination. Although the different failure modes have distinctive observable behavior, they are governed by the same fundamental failure process, namely damage initiation and propagation. A damage simulation approach, therefore, will be extremely valuable in improving understanding of, and predicting, material failure behavior, and in reducing experimental testing and hence accelerating material design, if it can predict damage initiation and propagation in different material phases and at different material length scales, as well as capture interaction and transition between different failure modes.

Damage involves surface separation, which can be characterized by crack initiation and growth. Various approaches have been developed to treat crack problems. In general, these approaches can be categorized into three categories according to their ability to predict crack initiation and growth in the presence or absence of pre-exiting cracks. The first category involves calculation of stress intensity factors or energy release rates analytically or numerically for a stationary crack. The second one involves modeling crack growth when crack exists in the 
analysis domain. And the third one involves predicting crack initiation and propagation with or without pre-existing cracks. These approaches and the related literature are described below.

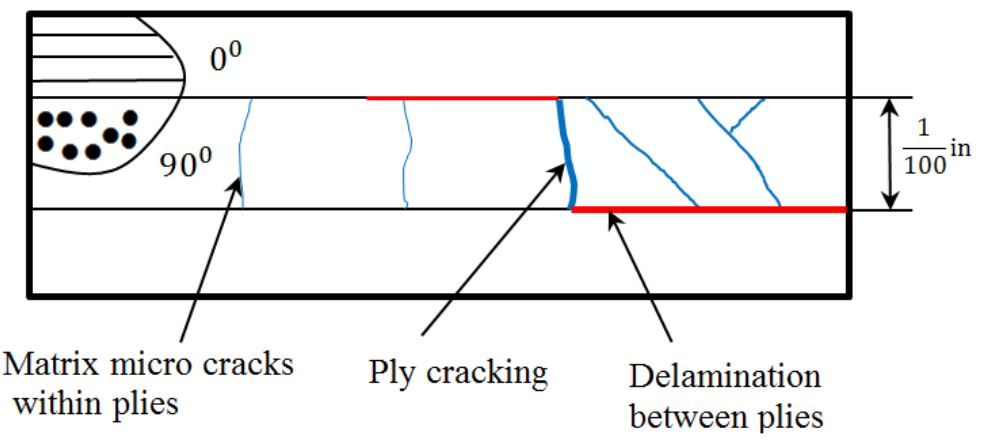

Figure 1.1 Schematic figure of damage in a laminated plate under thermal shock (reproduced from the image on the webpage of Center for Nondestructive Evaluation, ISU).

\subsection{Literature Review}

\subsubsection{Stress Intensity Factor and Energy Release Rate Calculations}

Cracks produce singular stress fields at the crack tips whose strength is characterized by stress intensity factors. Crack-face separation is typically described in terms of three modes. Mode I, the opening mode, is due to a tensile stress normal to the plane of the crack. Mode II, the sliding mode, is induced by shear stress acting parallel to the plane of the crack and perpendicular to the crack front. Mode III, the tearing mode, is caused by a shear stress acting parallel to the plane of the crack and parallel to the crack front. Linear elasticity theory can be used to determine the stress distribution around crack tips in polar coordinate, which typically takes the form:

$$
\sigma_{i j}(r, \theta)=\frac{K}{\sqrt{2 \pi r}} f_{i j}(\theta)+\text { higher order terms }
$$

where $K$ is the stress intensity factor and $f_{i j}$ is a dimensionless quantity that varies with the load and geometry. Once the actual stress distribution is obtained for a given problem, the corresponding stress intensity factors are determined by taking the limits below,

$$
\begin{gathered}
K_{I}=\lim _{r \rightarrow 0} \sqrt{2 \pi r} \sigma_{y y}(r, 0), \quad K_{I I}=\lim _{r \rightarrow 0} \sqrt{2 \pi r} \sigma_{y x}(r, 0), \\
K_{I I I}=\lim _{r \rightarrow 0} \sqrt{2 \pi r} \sigma_{y z}(r, 0)
\end{gathered}
$$

As an example, for an infinite plate under uniform far-field tension perpendicular to the crack face, the crack-tip stress distributions are obtained in closed form 


$$
\begin{gathered}
\sigma_{x}=\sigma \sqrt{\frac{a}{2 r}} \cos \frac{\theta}{2}\left(1-\sin \frac{\theta}{2} \sin \frac{3 \theta}{2}\right)(-\sigma) \\
\sigma_{y}=\sigma \sqrt{\frac{a}{2 r}} \cos \frac{\theta}{2}\left(1+\sin \frac{\theta}{2} \sin \frac{3 \theta}{2}\right) \\
\tau_{x y}=\sigma \sqrt{\frac{a}{2 r}} \sin \frac{\theta}{2} \cos \frac{\theta}{2} \cos \frac{3 \theta}{2} .
\end{gathered}
$$

from which the Mode I stress intensity factor $K_{I}$ is obtained as $\sigma \sqrt{\pi a}$. Similar results are obtained for the two remaining crack-opening modes. As discussed in the sequel, calculation of stress intensity factors using the elasticity approach is limited by the available analytical solutions. The propensity of a crack to propagate is defined by the critical stress intensity factor associated with a given crack-opening mode which is determined experimentally.

A related measure of a crack's propensity to propagate is the energy release rate which is the energy dissipated during fracture per unit of newly created fracture surface area. It is typically calculated by assuming a self-similar crack growth in the direction of the crack plane. Crack closure technique can be used to determine energy release rate if the release energy is only dissipated by newly created surfaces and not dissipated in other forms, such as sound, heat etc. The idea behind the crack closure technique is that if a crack extends by a small amount $\delta$, the energy released in the process is equal to the work required to bring the crack to its original length. After taking the limit of $\delta$, the released energy converges to corresponding energy release rate. When stress fields at the crack tip are available in closed form, energy release rates can be determined analytically. Figure 1.2 shows the closure of crack tip in an infinite plane under Mode I loading and associated crack extension in the crack's plane. For this loading the energy release rate may be calculated using the expression

$$
G_{I}=\lim _{\delta \rightarrow 0} \frac{2}{\delta} \int_{0}^{\delta} \frac{\sigma_{y} v}{2} d r
$$

where $\sigma_{y}$ is normal stress determined with original crack length and $v$ is vertical displacement determined in the configuration by assuming $\delta$ extension. Both of these have the functional form

$$
v=\frac{2 K_{I}}{E \sqrt{\pi}} \sqrt{2(\delta-r)}, \quad \sigma_{y}=\frac{K_{I}}{\sqrt{2 \pi r}}
$$



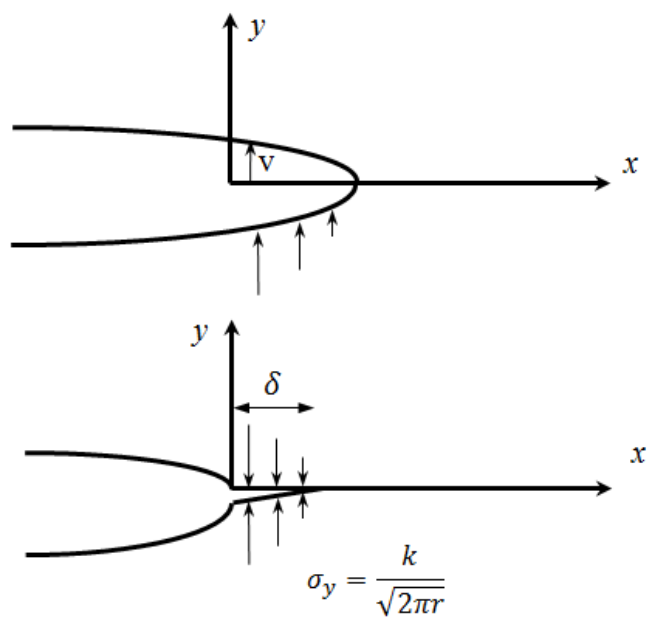

Figure 1.2 Closure of an extended crack tip.

$$
v=\frac{2 K_{I}}{E \sqrt{\pi}} \sqrt{2(\delta-r)}, \quad \sigma_{y}=\frac{K_{I}}{\sqrt{2 \pi r}}
$$

Employing the above expressions in the energy release rate equation and taking the limit of the crack extension going to zero, the following relation is obtained between the Mode I stress intensity factor and the energy release rate,

$$
G_{I}=\frac{K_{I}^{2}}{H}
$$

where $H=E /\left(1-v^{2}\right)$ for plane stress and $H=E$ for plane strain. Similar results are obtained under Mode II and Mode III loading.

The significance of the above result is that the energy release rate may be calculated from the knowledge of the stress singularity strength for a stationary crack once the crack-tip stress field is known.

\subsubsection{Analytical Approach}

A great deal of research work had been conducted on analytical solutions to crack problems since Griffith formulated the theory of brittle fracture using elastic strain energy concepts in 1920's, Griffith $(1921,1924)$. Generally speaking, the various analytical approaches are based on either the complex potential approach of Muskhelishvili's (1953a, b) or Fourier transform and series techniques employed by Sneddon (1951, 1961). In particular, complex potential approach is amenable to the treatment of this class of problem when a periodic array of cracks is along a single horizontal row or a single vertical column in an infinite homogenous 
medium, which can be effectively reduced to a single crack problem, Westergaard (1939), Koiter (1959), Sneddon and Srivastav (1965), Lowengrub (1966), as discussed by Sneddon and Lowengrub (1969). This approach was extended by Erdogan in 1962 to analyze two collinear interacting cracks, while Rice and Sih (1965) considered cracks along an interface separating two dissimilar half planes. Moreover, Delameter et al. (1975) analyzed doubly-periodic crack arrays by using the singular integral equation and Green's function approach within a unit cell framework based on periodic boundary conditions using complex potentials.

To conduct the analysis, a pre-existing crack is required and self-similar crack growth is usually assumed which allows calculation of the strain-energy release rate using the same solution form. As stated in the foregoing, self-similar crack growth means that the crack extends along the same crack front, producing similar stress and displacement fields in the original and extended crack configuration. However, these assumptions clearly cannot be used to predict nonself-similar crack growth. In those instances where self-similar crack growth is applicable, at least initially, such as delamination in multilayered media, the Fourier transform technique is an important technique that may be used to solve this type of problem. Erdogan and Gupta (1971) and Erdogan (1971) proposed a general procedure to analyze a single crack in a multilayered medium, which facilitated reduction of the problem to a system of singular integral equations governing the crack opening displacement components. This approach was extended by Chatterjee et al. (1982) and Chatterjee (1987) to deal with arbitrarily layered media with isotropic, orthotropic and monoclinic plies containing multiple cracks along different interfaces using the local/global stiffness matrix approach, Bufler (1971). Since considerable computational difficulties exist in treating interacting cracks, numerical results were limited to single delaminations due to limited computational resources in the 1970's and 1980's. Pindera (1991) applied this approach to analyze interlaminar stress distributions in a bi-material beam with two symmetrical positioned non-interacting disbonds under three-point bending and results were compared with experimental and finite-element results. Additional results were provided by Choi and Thangjitham (1994), who followed the work of Chatterjee et al. (1982), Chatterjee (1987), Pindera (1992).

In contrast, limited analytical work has been conducted in analyzing multiple crack interactions in homogenous or layered media with finite dimensions. Isida (1971) analyzed width and length effects of rectangular plates on the stress intensity factors of centrally positioned 
cracks by using Laurent series representation of complex potentials together with a boundary collocation procedure. By making use of quarter-range Fourier series representation of the displacement field in each layer and the local/global stiffness matrix approach, Chatterjee (1979) investigated two symmetric cracks in finite multilayered isotropic or orthotropic media pinned vertically at right and left ends under three point bending. Chen and Pindera (2007a, b) developed exact elasticity solutions for finite multilayered domains which can admit arbitrarily distributed interacting or non-interacting cracks parallel to the horizontal bounding surfaces at specified elevations or interfaces. In this work, Fourier series representation of the displacement field was employed that satisfied the Navier's equations for isotropic, orthotropic and monoclinic layers. Using this representation, a local stiffness matrix for each layer was constructed that related the harmonic coefficients of the surface displacements to the corresponding traction coefficients. This construction facilitated efficient satisfaction of traction and displacement continuity conditions between adjacent layers. To account for interfacial cracks, displacement discontinuity functions were introduced in terms of integrals of distributions of unknown functions. Satisfaction of traction and displacement continuity conditions produced the global system of equations, Eq. (1.8) that included the harmonics of the common displacements, $\bar{U}^{\mathrm{m}}$ and the corresponding displacement discontinuities $\overline{\mathrm{U}}^{\mathrm{m} *}$ across cracked interfaces.

$$
\mathbf{K}^{\mathrm{m}} \overline{\mathbf{U}}^{\mathrm{m}}=\overline{\mathbf{T}}^{\mathrm{m}}-\mathbf{L}^{\mathrm{m}} \overline{\mathbf{U}}^{\mathrm{m} *}
$$

where $\overline{\mathrm{U}}_{\alpha}^{m *(p)}=\frac{2}{m \pi} \int_{c_{\alpha}^{(p)}}^{d_{\alpha}^{(p)}} \hat{\xi}^{m} \Theta_{\alpha}^{(p)}\left(x^{\prime}\right) d x^{\prime}, \hat{\xi}^{m}$ is the matrix containing trigonometric functions and $\Theta_{\alpha}^{(p)}\left(x^{\prime}\right)$ is the displacement discontinuity density vector.

The problem can be reduced to a system of coupled singular integral equations with Cauchy-type kernels, Eq. (1.9), which describe the crack singularity exactly

$$
\begin{gathered}
\mathbf{T}_{\alpha}^{+}\left(x, \frac{h_{\alpha}}{2}\right)=\overline{\mathbf{A}}_{\alpha}^{*} \boldsymbol{\Theta}_{\alpha}^{(p)}(x)+\frac{1}{\pi} \int_{c_{\alpha}^{(p)}}^{d_{\alpha}^{(p)}} \overline{\mathbf{B}}_{\alpha}^{*} \frac{\mathbf{\Theta}_{\alpha}^{(p)}\left(x^{\prime}\right)}{x^{\prime}-x} d x^{\prime} \\
+\frac{1}{\pi} \sum_{\beta=2}^{n} \sum_{q=1}^{Q^{(\beta)}} \int_{c_{\beta}^{(q)}}^{d_{\beta}^{(q)}} \overline{\mathbf{K}}_{\alpha \beta}\left(x, x^{\prime}\right) \mathbf{\Theta}_{\beta}^{(q)}\left(x^{\prime}\right) d x^{\prime}+\mathbf{F}_{\alpha}(x)
\end{gathered}
$$

Where $\mathrm{n}$ in the summation limit is the total number of layers, $Q^{(\beta)}$ is numbers of cracks on the $\beta$ th interface, $\mathbf{T}_{\alpha}^{+}(x)$ is the traction vector specified on the bottom face of the $p$ th crack on the cracked $\alpha$ th interface, $\boldsymbol{\Theta}_{\alpha}^{(p)}(x)$ is the unknown displacement discontinuity density vector, 
$\overline{\mathbf{K}}_{\alpha \beta}\left(x, x^{\prime}\right)$ are regular Fredholm kernels, $\mathbf{F}_{\alpha}(x)$ is the specified external load vector, and $\overline{\mathbf{A}}_{\alpha}^{*}, \overline{\mathbf{B}}_{\alpha}^{*}$ are constant square matrices whose elements depend only on the material properties of adjacent layers.

To determine the unknown displacement discontinuity distribution functions, tractionfree condition on the crack faces was then employed, enabling determination of the displacement field in each layer in the presence of interfacial cracks.

The strain energy release rate caused by an infinitesimal crack extension of the $p$ th crack along the $\alpha$ th interface under self-similar crack growth is calculated by evaluating the integral given below

$$
\frac{\partial U_{\alpha}^{(p)}}{\partial a}=\lim _{\delta \rightarrow 0} \frac{1}{\delta} \frac{1}{2} \int_{ \pm 1}^{ \pm 1 \pm \delta^{\prime}} \frac{\left(d_{\alpha}^{(p)}-c_{\alpha}^{(p)}\right)}{2} \mathrm{~T}_{\alpha}^{+}\left(t_{\alpha}^{(p)}\right){ }^{\circ} \mathrm{U}_{\alpha}^{*}\left(t_{\alpha}^{\prime(p)}\right) d t_{\alpha}^{(p)}
$$

where the symbol ${ }^{\circ}$ denotes dot product operation, and the sign \pm refers to the right or left crack tip. The corresponding stress intensity factors at the right and left crack tips are given by the following formula

$$
K_{\alpha}^{p}=\lim _{t \rightarrow \pm 1}\left(-1 \pm t_{\alpha}^{(p)}\right)^{\frac{1}{2}} T_{\alpha}^{+}\left(t_{\alpha}^{(p)}\right)
$$

where $t_{\alpha}^{(p)}$ is the normalized coordinate measured from the $p$ th crack's centered.

The strength of the analytical approach is that stress intensity factor and energy release rate can be determined analytically by taking the limit as the crack extension tends towards zero. Quite often, however, it's very difficult to find analytical solutions for crack problems involving non-similar crack growth, crack interaction, complex geometries, boundary conditions and nonlinear material properties, which are common in actual applications.

\subsubsection{Numerical Approach}

With the emergence of numerical approaches, especially the finite-element approach, the stress and displacement fields in the analysis domain evolving complex geometries, material properties, boundary conditions usually can be accurately determined except for the area around the crack tip where large gradients due to singular stress fields occur. Hence in order to determine stress intensity factor and energy release rate, additional treatments are required. Two major approaches can be used to determine stress intensity factors and concomitant energy 
release rates: one is first to accurately determine the stress field using singular elements with built-in crack singularity and then extract stress intensity factors and energy release rates with different techniques, which will be discussed in this section. Another approach is directly based on the standard finite-element approach and makes use of the crack closure method proposed by Irwin (1958). In contrast to the analytical expression, Eq. (1.4), of calculating energy release rate, Eq. (1.12) is the numerical implementation and various techniques have been developed to implement the crack closure method, which is can be easily adapted to simulate crack growth and will be discussed later in section of 1.2.2.

$$
E R R=\lim _{\Delta_{a} \rightarrow 0} \frac{1}{2 \Delta_{a}} \int_{a}^{a+\Delta_{a}} T_{i} \Delta u_{i} d s
$$

where $T_{i}$ are stresses extracted from the elements ahead of the crack tip in the range of [a $a+$ $\Delta_{a}$ ] by keeping the original crack length and $\Delta u_{i}$ are displacements determined in the range of [ $a$ $a+\Delta_{a}$ ] by assuming $\Delta_{a}$ extension towards the original crack.

To calculate the stress intensity factor, the first step is to generate accurate singular stress field and after that proper techniques need to be used to determine its value. It is known that the standard finite-element approach will only produce finite stress at the crack tip and this makes it difficult to extract the crack-tip singular behavior. To overcome this disadvantage, researchers developed singular crack-tip elements, (Byskov 1970, Walsh 1971, Tracey 1971), which incorporate singular stress behavior into the shape functions. The disadvantage of this approach, however, is the extra work involved in embedding singular elements into regular finite-element meshes.

Subsequently, Barsoum (1974) and Henshell and Shaw (1975) proposed quarter-point singular elements based on isoparametric elements, Figure 1.3, to overcome the disadvantage. The pioneering work of Barsoum (1974) and Henshell and Shaw (1975) stimulated extensive subsequent work as described in two comprehensive reviews by Banks-Sills (1991, 2010), and now the capability is also available in commercial finite-element packages, such as Abaqus and ANSYS. After obtaining accurate singular stress and corresponding displacement fields, the next step is to extract the stress intensity factor, which can be obtained via four approaches: displacement extrapolation, the stiffness derivative, J-integrals and M-integrals. 


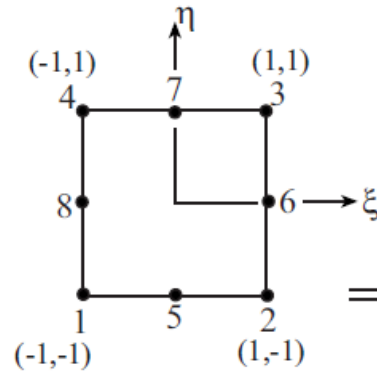

(a)

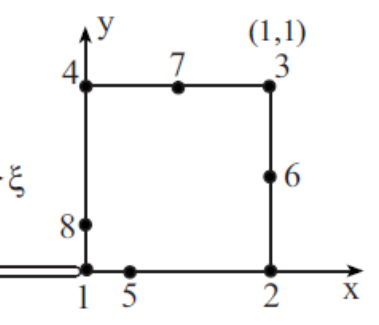

(b)

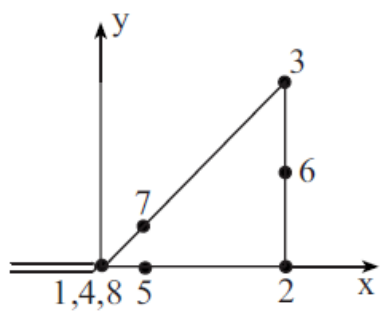

(c)

Figure 1.3 Eight-node: (a) parent element in $\eta-\xi$ plane, (b) quarter-point element in the $x-y$ plane and (c) Quarter-point collapsed triangular element.

The first approach is known as a direct method and the other three are indirect methods based on energy quantities. With the displacement extrapolation method, (Chan et al. 1970, Banks-Sills and Einav 1987), stress intensity factor is found by extrapolating the displacement along the crack face and linear regression may be employed to determine the value. The extrapolation operation does not guarantee results' accuracy, and therefore this method is considered less accurate than other three energy-based indirect approaches, Banks-Sills (1991). On the other hand, this approach is easy to implement and can be used to calculate stress intensity factors for mixed-mode problems as well, (Banks-Sills et al. 2005, Freed and BanksSills 2005). Regarding the energy approaches, virtual crack extension is required to assist with the generation of some derivative terms, which is a very small length on the order of $10^{-6}$ of a characteristic element length (Banks-Sills 1991) and the singular element with virtual extension retains the character of quarter-point (Banks-Sills 1989).

Towards the calculation of pure mode I stress intensity factor via indirect approach, Jintegral energy needs to be determined, which was originally presented by Rice (1968) for 2D geometries as

$$
J=\int_{\Gamma} W n_{1}-T_{i}\left(\partial u_{i} / \partial x_{1}\right) d s
$$

where $\Gamma$ is any path beginning at the lower crack face, encircling the crack tip and ending at the upper crack face as shown in Figure 1.4. And two approaches can be used to determine J-integral energy: one approach is the stiffness derivative technique proposed by Parks (1974) and another one is the technique suggested by $\mathrm{Li}$ et al. (1985), in which J-integral is expressed via a set of basis functions along the crack front. For linear elastic materials or materials that experience 
small-scale yielding at crack tip, J-integral is equal to strain energy release rate and they can be related to mode I stress intensity factor via Eq. (1.7).

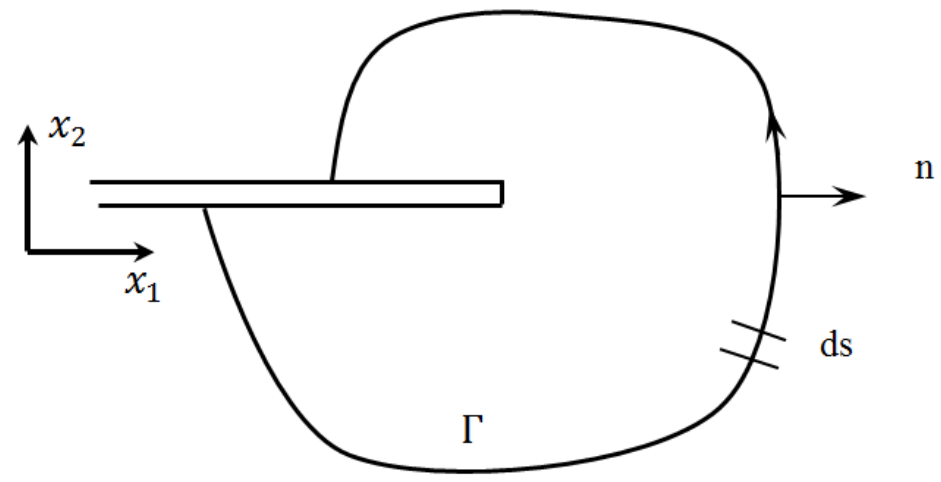

Figure 1.4 Example of line J-integral path.

From the above discussion we can see that the J-integral, which is a single value, can only be directly related to one type of stress intensity factor instead of three different stress intensity factors, $K_{I}, K_{I I}, K_{I I I}$, which exist when the crack is under mixed-mode deformation. Mixed mode stress intensity factors can be separated via the M-integral, which was first derived by Chen and Shield (1977) based on the J-integral and implemented by Yau et al. (1980), see a comprehensive review provided on this topic by Banks-Sills (2010).

\subsubsection{Crack Growth Simulation}

One of the most common failure modes for composites structures is delamination, (Garg 1988, Bolotin 1996, Pagano and Schoeppner 2000). Over the past two decades, fracture mechanics approaches have been extensively used to characterize the onset and growth of delamination, (O’Brien 1982, 1998, Martin 1998). In particular, Rybicki and Kanninen (1977) proposed the Virtual Crack Closure Technique (VCCT), which is widely used for computing energy release rate and predicting crack propagation based on 2D or 3D finite element results due to its simplicity in methodology and ability in handling mixed-mode failure, (Raju 1987 and Buchholz et al. 1988). It is necessary to keep in mind, however, that this approach requires pre-existing cracks in the analysis domain.

In general, numerical implementation of the crack closure method may be divided into three categories. The first one is to take the limit numerically for the released energy normalized by different crack extensions, in which the multiple normalized release energy rates are 
calculated based on different crack extension lengths. Subsequently, the sought energy release rate is obtained by extrapolating the normalized released energy rates to the point $\Delta_{a}=0$. This approach was employed using FVDAM to determine the energy release rates of a crack propagating along the fiber/matrix interface, Tu et al. (2012). Figure 1.5 shows the unit cell discretization and the initial crack configuration. To determine the energy release rate at each crack length around the interface, released energy was calculated for different crack extensions, Figure 1.6, and the asymptotic limit was obtained by extrapolation to the point of $\Delta_{a}=0$. Figure 1.7 shows the distribution of energy release rates for different crack lengths and the distribution indicates that the crack is likely to get arrested around $\pm 65^{\circ}$. This observation is consistent with the results obtained by Tu and Pindera (2014) based on the CZM-based simulations described in Chapter 4.

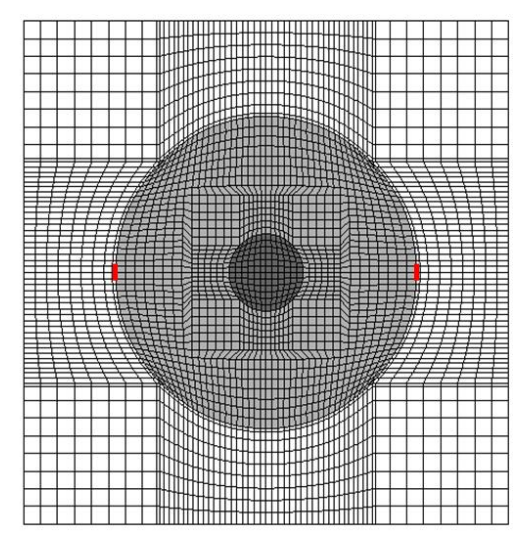

Figure 1.5 Discretization of unit cell and initial crack distribution with small crack.

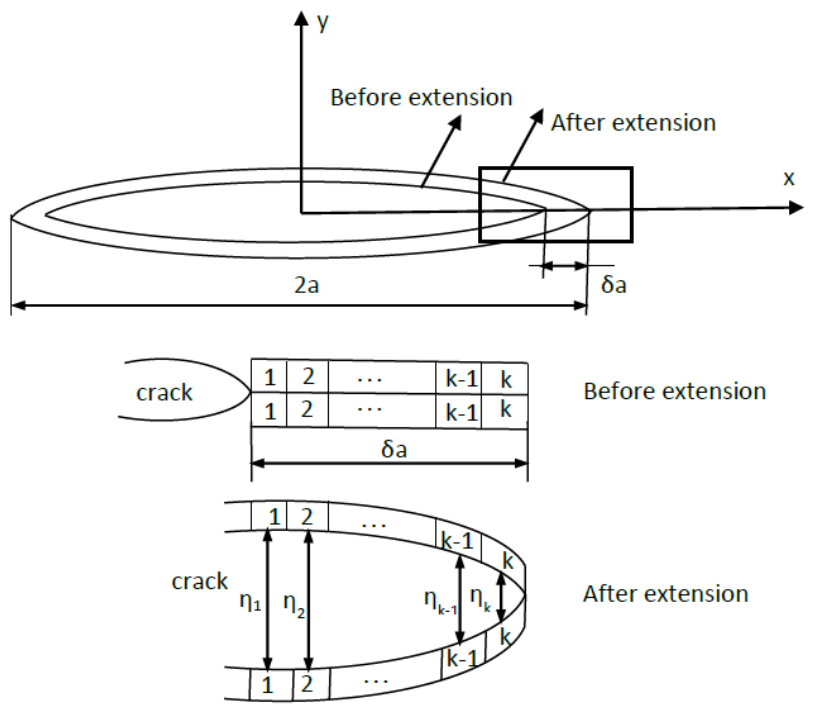

Figure 1.6 Illustration of crack extension with different length. 


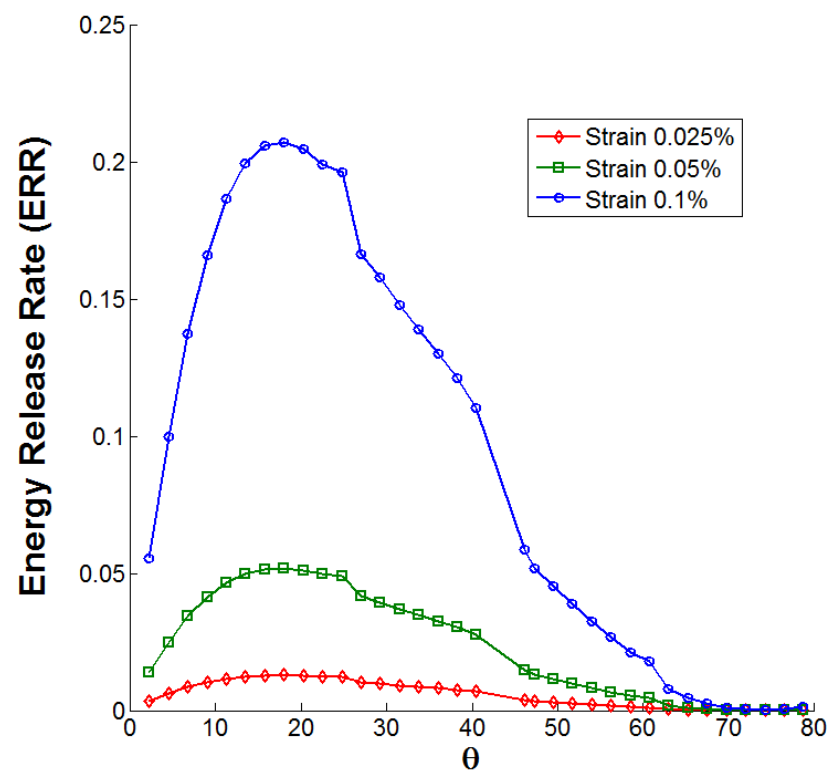

Figure 1.7 Energy release rate for different crack lengths.

The second one is to use two analysis steps (Broek 1991 and Krueger 2002) as discussed by Kruger (2002), in which the released energy $\Delta E$ is equal to the energy required to close the crack extension $\Delta a$ from the original crack length a to crack length $a+\Delta a$ and energy release rate is equal to the released energy $\Delta E$ divided by crack extension $\Delta a$. In this approach, one assumption is implied that $\Delta a$ is small comparing with crack length. And the third approach, VCCT, is modified based on the second approach and only one analysis step is required, in which the released energy is calculated based on reaction forces at the crack tip and nodal displacements just ahead of the crack tip. Also this approach implies crack extension is small and it does not cause changes of stress and displacement fields around the crack tip. A sensitive study conducted by Rybicki and Kanninen (1977) shows that for the problem of a finite plate with a central crack, accuracy of the VCCT's results is within $6 \%$ of the reference solution when the crack extension $\Delta a$ is up to $20 \%$ of the crack length.

VCCT does not employ assumptions on the functional form of stresses and displacements and the regular finite-element approach is used. However, with the availability of singular quarter-point elements which capture the crack-tip stress field singularity, VCCT also has been incorporated into the framework of quarter-point elements to determine the energy release rate, 
first proposed by Raju (1987), Ramamurthy et al. (1986) and later followed by Narayana et al. (1990), Narayana and Dattaguru (1996), Sethuraman and Maiti (1988). Because quarter-point elements are not readily available in many finite-element codes and the quarter-point elements cannot be easily deployed to simulate crack growth because of changing crack tip position during the crack growth process, the major developments and applications of VCCT are limited to the regular finite-element method. The discussion in this dissertation will also be confined to the regular finite-element approach. VCCT has been extensively implemented into 2D and 3D frameworks and became available in commercial finite-element software such as Abaqus, ANSYS and Marc.

Regarding the method's usage in real practice, Krueger (2002) provided the insights summarized below: 1) For geometric nonlinear analysis, both tractions and displacements used in calculating the energy release rates need to be transformed to a local coordinate system. 2) Original paper written by Rybicki and Kanninen (1977) imposed the assumption that element lengths for the elements in front and behind the crack tip are identical, which restricts the application of the approach in practice since in complex models equal element size cannot be easily obtained, especially when modeling crack propagation. To overcome this disadvantage, correction factors are used in the formula for energy release rate calculation by taking into account the element lengths in front and behind the crack tips, with similar approach in treating 3D crack fronts. 3) Extra care needs to be taken in obtaining the energy release rate using VCCT when the crack is situated along a bi-material interface, (Raju et al. 1988, Hwu and Hu 1992, Sun and Jih 1987, Sun and Manoharan 1989) because of stress oscillation at the crack tip. To overcome this problem, one way is to insert a resin rich layer between the adjacent plies, (Raju et al. 1988 and Dattaguru et al. 1994). However, this requires significant refinement in the thin resin layer, which increases the model size dramatically. Another more realistic way is to control the element size in from and behind the crack front, which should be small enough to assure a converged finite-element solution but large enough to avoid oscillatory behavior, (Krueger 1994, Sun and Qian 1997).

From the above review of the crack closure technique implemented within the finiteelement framework, we can see that this approach is powerful and versatile in calculating energy release rates and treating crack propagation problems. However, this approach has some intrinsic shortcomings. First, the approach cannot predict crack nucleation and thus the analysis domain 
has to have pre-existing cracks, which is often not true in reality. Another problem is that the length of pre-existing cracks is important, and hence cannot be arbitrary, since the majority of numerical implementations are based on the assumption that the crack extension does not alter the crack-tip stress fields. Moreover, the mesh in front the crack tip cannot be arbitrary, requiring extra attention.

\subsubsection{Crack Initiation and Propagation Simulation}

To naturally track the evolution of localized failure modes, the cohesive zone model proposed by Barenblatt $(1959,1962)$ for fracture of brittle materials, and subsequently extended by Dugdale (1960) for perfectly plastic materials, has been adopted by numerous researchers during the past 30 years. The model is based on a traction-interfacial separation relation which describes the interfacial degradation process between two adjacent phases or subdomains. As the interface separates, traction first increases until the interfacial strength is reached, and then decreases to zero, where complete separation occurs. The contributions of Needleman and coworkers (1987, 1994), and Ortiz and coworkers (1993, 1996), have spurred extensive use of this model in simulating fracture phenomena in a wide range of materials due to its ability to mimic spontaneous crack nucleation, crack branching and fragmentation, as well as crack propagation without an external fracture criterion, and in the absence of self-similar crack growth. Reviews of the different aspects and applications of the cohesive zone model have been provided by Elices et al. (2002), Banea et al. (2009), and Park and Paulino (2011).

In order to overcome the mesh dependence in finding non-smooth solutions involving discontinuities, singularities, high gradients etc., enrichment functions have been introduced into the classic finite-element approaches, (Belytschko et al. 2000, Zienkiewicz and Taylor 2000), which were customized to capture jumps, singularities, etc. This approach has two different names: one is known as the generalized finite-element method (GFEM) which was adopted by the Texas school in 1995-1996, (Duarte and Oden 1996, Melenk and Babuska 1996, Melenk 1995) and another name is the extended finite-element method (XFEM), which was coined by the Northwestern school in 1999, (Moes et al. 1999, Belytschko and Black 1999). GFEM/XFEM may be used to analyze stationary cracks and model crack initiation and propagation. Different forms of the asymptotic crack-tip functions were discussed by Sukumar et al. (2004), Sukumar and Prevost (2003), and Elguedj et al. (2006). However, accurate modeling of the crack-tip 
singularity can be inconvenient using this approach since the degree of crack singularity depends on the crack location in a non-isotropic material, which may change during the crack propagation process, (Abaqus 6.14 Manual). One alternative approach within the GFEM/XFEM framework to avoid modeling the crack-tip stress singularity is to use the traction-separation behavior which describes crack initiation and propagation. Different from the cohesive zone model in the classic finite-element approach, which requires the cohesive surface to align with element boundaries and the crack to propagate along pre-defined paths, the GFEM/XFEM-based cohesive segments method allows cracks to initiate and propagate along an arbitrary, solution-dependent path in the bulk materials since crack propagation is not tied to the element boundaries in a mesh. Song (2006) and Remmers (2008) have used this approach to simulate crack initiation and propagation of multiple cracks in solids, which provides an effective and attractive engineering method and now is available in the commercial finite-element package, Abaqus. Currently, the development of XFEM/GFEM is still in its early stage and many promising applications remains, (Belytschko et al. 2009).

\subsection{Finite-Volume Theories in Solid Mechanics Applications}

From the above literature review, we see that the cohesive zone model has demonstrated its unique capability in simulating complex fracture phenomena either with or without preexisting cracks, which intrinsically exist at different length scales and ultimately lead to failure of composite materials. While the majority of cohesive zone model applications are based on variational techniques, it is only relatively recently that the cohesive zone model has been incorporated into finite-volume based techniques to simulate damage evolution.

The finite-volume method has proved an attractive alternative to the established finiteelement analysis of boundary-value problems in solid mechanics, (Berezovski et al. 2008) following its origins in fluid mechanics, (Leveque 2002, Versteeg and Malalasekera 2007). In contrast to variational techniques, local equilibrium is satisfied in integral sense at the discretized subdomain level in the finite-volume method, offering solution stability and other advantages, Cavalcante et al. (2012). There are three variants of finite-volume methods that can be identified in the analysis of solid mechanics problems: the cell-centered finite-volume technique, the cellvertex finite-volume techniques and the surface averaging finite-volume techniques. These 
variants of the finite-volume method differ in the manner of analysis domain discretization, subdomain field variable approximation and solution technique as described below.

Similar to the finite-volume method employed in fluid mechanics, the cell-centered finite-volume method employs control volumes which are centered around grid points at which field variables are defined, Figure 1.8a. In the early development stage, in order to satisfy equilibrium equations at the discretized volume level in an integral sense, Taylor series expansion was utilized to approximate surface variables in terms of the control volume-center variables, (Demirdzic et al. 1988, Demirdzic and Martinovic 1993, Demirdzic and Muzaferija 1994). Linear displacement field approximation along control volume faces was implemented by Wheel $(1996,1999)$ in solving axisymmetric linear and incompressible elasticity problems. Recently, Falla (2006, 2008) introduced shape functions into the cell centered finite-volume framework in conjunction with parametric mapping in the solution of plane problems base on the Mindlin-Reissner plate theory. This practice provided a better approximation of the variation of unknown variables across control volume faces, which facilitate calculation of interfacial stress resultants.

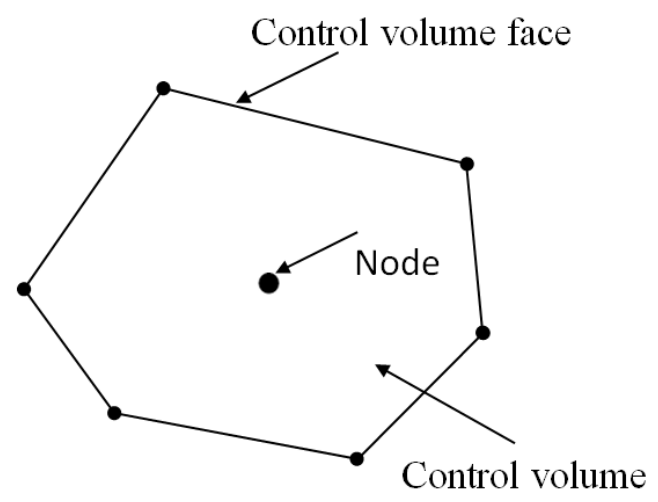

(a) Cell centered control volume

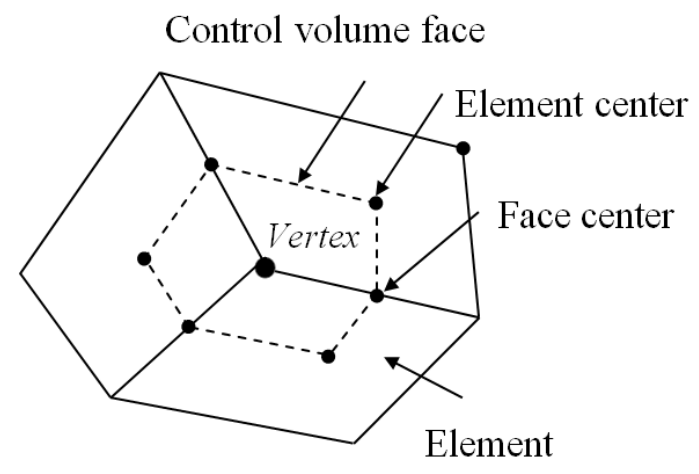

(b) Vertex based control volume

Figure 1.8 Control volumes employed in cell centered and vertex based finite-volume analyses of homogeneous material and structural problems in solid mechanics.

The cell-vertex finite-volume approach leverages elements of the finite-element method in domain discretization and displacement field approximation. The analysis domain is first discretized into elements, and the field variables are defined at the common vertices of adjacent elements using shape functions borrowed from the finite-element approach. Control volumes center around the vertices are constructed by using element and face centers as control volume corners, Figure 1.8b. Thus the control volume geometry and displacement field approximation 
are directly linked to element discretization and shape functions and the local equilibrium equations can be satisfied over all control volumes. This approach was first proposed by Fryer et al. (1991) for the analysis of 2D elasticity problems and subsequently extended to 3D problems by Bailey and Cross (1995), and then applied by Taylor et al. (1995, 2003), Fallah et al. (2000), Wenke and Wheel (2003), Wheel (2008) and Pan et al. (2010).

The research based on cell-centered and vertex-based finite-volume methods has demonstrated that the finite-volume method is a very viable alternative to the finite-element approach in the solution of structural and solid mechanics boundary-value problems involving at least homogeneous materials. Following the success of CZM in finite-element applications, CZM has recently been implemented into the cell-centered finite-volume framework, (Stylianou and Ivankovic 2002) and applied to study crack propagation along a bi-material interface (Carolan et al. 2013).

The third variant of the finite-volume method has been developed independently of the above two approaches to model materials with heterogeneous microstructures, (Suquet 1987, Charalambakis and Murat 2006, Buryachenko 2007, Birman and Byrd 2007, Chatzigeorgiou et al. 2008, and Paulino et al. 2008). It is rooted in so-called Higher-Order Theory for Functionally Graded Materials (HOTFGM), developed in a sequence of papers in the 1990's and summarized in Aboudi et al. (1999). The homogenized counterpart was constructed by Aboudi et al. (2001), which was subsequently renamed as the High-Fidelity Generalized Method of Cells (HFGMC) by Aboudi et al. (2002) although predictions of HFGMC generally do not reduce to those of the Generalized Method of Cells (GMC), (Paley and Aboudi 1992), as expected of theories related to each other through similar names. By simplifying the discretization of analysis domain which, in turn, facilitated implementation of the efficient local/global stiffness matrix approach (Bufler 1971, Pindera 1991), the structural and homogenized versions of these so-called higher-order theories were subsequently re-constructed in a sequence of papers by Bansal and Pindera (2003, 2005) and Zhong et al. (2004). This significant re-construction revealed the above higher-order approaches to be in fact finite-volume theories. To faithfully reflect the fundamental character of these re-constructed theories, the version of the finite-volume theory developed for periodic materials within the homogenization framework was subsequently named finite-volume direct averaging micromechanics (FVDAM) theory, Bansal and Pindera (2006). The re-constructed finite-volume theories are similar to the cell-centered techniques that evolved in parallel for 
homogeneous materials and structures during roughly the same period. However, in contrast with the cell-centered techniques, the re-constructed methods utilize explicit displacement field approximation within individual subvolumes and apply both interfacial displacement and traction continuity conditions at the same time in a surface-averaged sense, following the original idea proposed by Achenbach (1975). This leads to the satisfaction of equilibrium equations in a surface-averaged sense and the explicit construction of local stiffness matrix for the individual subvolumes following the elasticity approach.

To enable efficient modeling of complex microstructures, parametric mapping was incorporated into these re-constructed theories. This mapping capability was first introduced by Cavalcante (2006) and Cavalcante et al. (2007a, b) into the structural version of the finitevolume theory originally developed by Bansal and Pindera (2003) and Zhong et al. (2004). Subsequently, following the work of Cavalcante et al. (2007a, b), Gattu et al. (2008) and Khatam and Pindera (2009a, b) introduced parametric mapping into the rectangular subvolume-based version of the FVDAM theory originally developed by Bansal and Pindera (2005, 2006). Both the structural and homogenized versions of the parametric finite-volume theory have proved to be attractive alternatives to the finite-element analysis of heterogeneous materials and the predictive capability of the reconstructed theory has been verified both analytically, numerically and experimentally. In particular, the accuracy of homogenized and local responses has been shown to be comparable to the finite-element method with the added advantage that explicit relations between surface-averaged tractions and displacements are available through a local stiffness matrix that governs the equilibrated response of a subdomain. This circumvents the problem of accurate stress extrapolation to the subdomain surfaces encountered in other finitevolume as well as finite-element approaches. The analytical relations between interfacial tractions and interfacial displacements provide a natural way to incorporate cohesive zone model into the FVDAM framework absent in other approaches. This and other features of FVDAM set it apart from the other two variants of the finite-volume method, Pindera et al. (2009). Herein, we refer to this version of the parametric FVDAM theory as standard parametric FVDAM theory.

However, interfacial interpenetration and discontinuity of the non-traction stress components are shortcomings which remained to be addressed in the standard parametric FVDAM theory, Cavalcante et al. (2012a). Nonetheless, the subvolume average values of both traction and non-traction stress components are reliable (Cavalcante et al., 2008, 2011), as 
reported by Katham and Pindera in their papers. To overcome the above shortcomings of the standard parametric FVDAM theory, a generalized finite-volume theory has been developed by Cavalcante and Pindera (2012a, b) based on rectangular analysis subdomain for elasticity analysis that employs a higher-order displacement field representation within individual subvolumes of a discretized analysis domain, in contrast with the second-order expansion employed in the 0th order theory. The predictive capability of the generalized theory has been further demonstrated by analyzing diverse problems involving finite deformation, viscoelastic and elastic-plastic analyses, (Cavalcante and Pindera 2014a, b, Cavacante and Marques 2014, Cavalcante and Pindera 2016). Most recently Chen et al. (2016) extended the rectangular version of FVDMA theory developed by Bansal and Pindera $(2005,2006)$ to three dimensions based on cubic subdomains and the developed theories also incorporated the Bodner-Partom (1975) constitutive model, which is a state-variable-based viscoplastic model.

\subsection{Objectives}

The overall objective of the proposed work is to produce a unique and novel finite-volume based homogenization theory for the response of unidirectionally-reinforced periodic materials with evolving damage and optimization capabilities. This theory will take the form of generalized Hooke's law that simulates damage initiation and growth under combined three-dimensional loading in the macroscopic strain or stress spaces. Relatively little work has been done in constructing a homogenization theory leading to the homogenized Hooke's law valid under combined three-dimensional loading which takes into account the various damage modes, their evolution and transition. In contrast with commercial finite-element codes such as Abaqus or Ansys, the three-dimensional loading capability will be intrinsic to the theory, requiring only specification of the loading path in terms of macroscopically applied strains or stresses. In commercial finite-element codes, such capability is accomplished by imposing appropriate boundary conditions on the unit cell representative of the simulated material's mirostructure on a case-by-case basis. The boundary conditions in the proposed theory will be an integral part of the theoretical framework and hence transparent to the user.

The proposed theoretical framework is semi-analytical, with a straightforward input data construction. This enables automated manipulation of the input data in parametric or optimization studies. To leverage this capability in a number of applications, ranging from 
material architecture designs that meet targeted performance to material parameter identification that may otherwise be difficult to determine experimentally, the proposed homogenization theory will be incorporated into a robust optimization algorithm and demonstrated through relevant examples.

Finally, upon validation, the homogenization theory will be employed to revisit classical and emerging problems to demonstrate its capabilities and to address important and unanswered questions. This includes damage model bifurcation in symmetric cross-ply laminates and the relationship between homogenized response features at the macroscale and the underpinning damage mechanisms.

\subsection{Methodology}

The construction of the generalized Hooke's law with evolving damage capability is based on the standard parametric FVDAM theory of Khatam and Pindera (2009 a, b). This version was the most updated one when the author initiated his work whilst the development of the generalized version of FVDAM was taking place in parallel. Although interfacial interpenetration and discontinuity of non-traction stress components occur in the standard parametric FVDAM theory, (Cavalcante et al. 2012), the surface-averaged values of both traction and non-traction stress components are reliable, Cavalcante et al., (2008, 2011), especially if the heterogeneous materials are not subjected to large deformation, Khatam and Pindera (2012), which is the scenario of interest herein.

The incorporation of damage capability into the parametric FVDAM theory is carried out using a unified approach that allows simulation of both progressive phase separation based on the cohesive zone model as well as crack presence and growth within the same framework. To accomplish this, displacement discontinuity functions $\widehat{\mathbf{U}}_{*}^{\prime}$ previously used in the solution of interfacial crack problems in multilayered materials, (Chen and Pindera 2007a, b), are first introduced into the FVDAM framework. As in the exact elasticity framework of Chen and Pindera (2007a, b), the introduction of these functions modifies the global system of equations for the unknown surface-averaged interfacial displacements, called the primary system of equations, which is obtained through the satisfaction of interfacial displacement and traction continuity conditions,

$$
\mathbb{k} \widehat{\mathbf{U}}_{o}^{\prime}=\Delta \mathbf{C} \bar{\varepsilon}+\Delta \Gamma \Delta T-\mathbb{L} \widehat{\mathbf{U}}_{*}^{\prime}
$$


In the above, $\widehat{\mathbf{U}}_{o}^{\prime}$ and $\widehat{\mathbf{U}}_{*}^{\prime}$ are common surface-averaged fluctuating displacements and displacement discontinuities, respectively, $\mathbb{k}$ is the global stiffness matrix containing information on subvolume geometry and material assignment, the matrix $\Delta \mathbf{C}$ is comprised of differences in material stiffness matrices of adjacent subvolumes, $\mathbb{L}$ is the matrix that represents contributions from damaged or cracked interfaces, and $\bar{\varepsilon}$ is the applied (specified) homogenized strain that represents loading.

The discontinuity functions are obtained upon solution of auxiliary equations that represent either traction-free crack face conditions along interfaces with existing cracks, or interfacial degradation between phases governed by nonlinear traction-interfacial separation laws along interfaces undergoing damage

$$
\mathbf{T}=\mathbf{K}_{*} \widehat{\mathbf{U}}_{*}^{\prime}+\mathbf{K}_{o} \widehat{\mathbf{U}}_{o}^{\prime}+\mathbf{C} \overline{\boldsymbol{\varepsilon}}+\boldsymbol{\Gamma} \Delta T
$$

In contrast with the exact elasticity approach of Chen and Pindera (2007 a, b) which describes the crack tip singularity exactly by reducing the related system of equations to a system of coupled Cauchy-type singular integral equations, in the present approach the above system of equations is solved directly for the unknown displacement discontinuity functions as described below.

For stationary cracks, the left-hand side of the equations is set to zero which enables direct solution of $\widehat{\mathbf{U}}_{*}^{\prime}$ in conjunction with Eq. (1.14). The penalty is extensive crack-tip mesh refinement needed to capture the stress singularity accurately enough for an accurate energy release rate calculation. This is offset by much greater flexibility than the elasticity approach since the proposed approach does not have limitations on crack distributions, model geometry and boundary conditions.

Alternatively, for interfaces undergoing damage evolution and ultimately separation, the cohesive zone model is introduced which provides traction-displacement separation relation to describe crack initiation and growth. The traction vector on the right-hand side of Equation (1.15) is replaced by the nonlinear separation law represented by the cohesive zone model. As observed above, the introduction of displacement discontinuity functions directly facilitates the implementation of cohesive laws since the interfacial tractions are available in terms of explicit functions of the displacement discontinuity. The two-level global system of equations includes the primary global system of equations, which only needs to be solved once during the analysis process and the global auxiliary system of equations which only contains the degrees of freedom 
associated with the cohesive interfaces. This system is solved iteratively and incrementally during the process of modeling interfacial non-linear behaviors.

In contrast with the manner of incorporating CZM into the finite-element framework where nodal displacements are the basic unknowns, within the FVDAM framework the surfaceaveraged displacements at subvolume interfaces undergoing evolving damage are expressed as sums of continuous displacements and displacement discontinuities. The explicit relations between interfacial tractions and displacements available through the local stiffness matrix formulation enable us to construct the auxiliary system of equations directly. This manner of reformulating the governing system of equations for the unit cell response provides two very attractive advantages. As already mentioned, the incorporation of CZM is straightforward due to the explicit traction-interfacial separation relations. Moreover, in order to obtain converged solutions for the displacement discontinuities, only the auxiliary system of equation needs to be solved iteratively, unlike the finite-element framework where the entire global system is solved at each load increment. The number of auxiliary equations is equal to the number of interfaces undergoing damage multiplied by the associated degrees of freedom. When this number is small compared to the total number of interfaces within the discretized unit cell, as in the case of interfacial debonding in fiber-reinforced materials, the auxiliary system of equations is also small facilitating very efficient solution.

The robust optimization algorithm chosen for use in conjunction with the extended FVDAM is the Particle Swarm Optimization (PSO) algorithm. This is a non-gradient based technique that is well-suited for optimization problems involving objective functions that vary non-smoothly with design variable changes, thereby providing robustness in a wide class of optimization problems. The utility of the developed FVDAM-PSO algorithm is illustrated by identification of optimal CZM interface parameters, which are difficult to measure experimentally, in the simulation of interfacial fiber/matrix debonding in a unidirectional metalmatrix composite. This capability can be very attractive for material design, especially nowadays with the rapid advancement in 3D printing techniques.

The extended FVDAM theory is verified using modified Eshelby problems under inplane tensile and out-of-plane shear loading when interfaces are within the linearly elastic range. Its capability in simulating crack initiation and propagation is critically evaluated upon comparison with the results generated using the commercial finite-element code Abaqus. 
Experimental validation is provided through comparison of the FVDAM simulations with experimental response of unidirectional $\mathrm{SiC} / \mathrm{Ti}$ composites undergoing fiber/matrk debonding as well as damage evolution in polymeric matrix cross-ply laminates.

\subsection{Outline of Dissertation}

The dissertation is organized as follows. In Chapter 2, the 0th-order parametric FVDAM theory is further extended in order to model the evolution of damage in periodic heterogeneous materials using the displacement discontinuity function techniques. The cohesive zone model $(\mathrm{CZM})$ is then implemented to simulate progressive separation of adjacent phases or subdomains. The new capability is verified in the linear region upon comparison with an exact elasticity solution for an inclusion surrounded by a linear interface of zero thickness in an infinite matrix that obeys the same law as CZM before the onset of degradation. In Chapter 3, critical evaluation is conducted regarding the interfacial debonding simulation of fiber-reinforced composite materials with FVDAM and Abaqus. In Chapter 4 the extended theory's utility is demonstrated by revisiting the classical fiber/matrix debonding phenomenon observed in $\mathrm{SiC} / \mathrm{Ti}$ composites and its ability to accurately capture the mechanics of progressive interfacial degradation is illustrated. In Chapter 5, the extended theory is incorporated into Particle Swarm Optimization and the optimal interfacial properties are identified effectively. In Chapter 6, the classical phenomenon of progressive cracking of $90^{\circ}$ plies in polymeric matrix cross-ply laminates, and potential or subsequent delamination along the $0^{\circ} / 90^{\circ}$ ply interface, is critically revisited using a finite-volume homogenization theory with damage evolution capability. In Chapter 7, damage evolution in off-axis laminates is investigated. Summary and conclusion, and a discussion of possible future work are presented in Chapter 8. 


\section{Chapter 2}

\section{Cohesive Zone Model-Based Finite-Volume Homogenization Theory}

\subsection{Introduction}

In this chapter, we extend the parametric FVDAM theory using a unified approach that allows simulation of both progressive phase separation based on CZM and crack growth within the same framework. To accomplish this, displacement discontinuity functions previously used in the solution of interfacial crack problems in multilayered materials, (Chen and Pindera 2007a), are introduced into the FVDAM framework. The discontinuity functions are obtained upon solution of auxiliary equations that represent either traction-free crack face conditions, or interfacial degradation between phases governed by nonlinear traction-interfacial separation laws. The approach is verified in the linear range upon comparison with an exact elasticity solution for the problem of an interface-clad cylindrical inclusion embedded in an infinite matrix under far-field unidirectional loading and out-of-plane shear loading.

\subsection{Theoretical Framework}

The unit cell's microstructure is discretized into quadrilateral subvolumes designated by the index $(q)$ whose location is specified by the subvolume vertices $\left(y_{2}^{(p, q)}, y_{3}^{(p, q)}\right)$. The $q$ th quadrilateral subvolume is generated by mapping the reference subvolume in the $\eta-\xi$ plane bounded by $-1 \leq \eta \leq 1$ and $-1 \leq \xi \leq 1$ onto its actual location in the unit cell, Figure 2.1, using the transformation

$$
y_{i}^{(q)}(\eta, \xi)=\sum_{p=1}^{4} N_{p}(\eta, \xi) y_{i}^{(p, q)}, \quad i=2,3
$$

where $N_{1}(\eta, \xi)=\frac{1}{4}(1-\eta)(1-\xi), N_{2}(\eta, \xi)=\frac{1}{4}(1+\eta)(1-\xi), N_{3}(\eta, \xi)=\frac{1}{4}(1+\eta)(1+\xi)$ and $N_{4}(\eta, \xi)=\frac{1}{4}(1-\eta)(1+\xi)$. Following the convention of Cavalcante et. al. (2006), the 
subvolume's vertices are numbered in counterclockwise manner starting from lower left $\operatorname{corner}\left(y_{2}^{(1, q)}, y_{3}^{(1, q)}\right)$. Accordingly, the faces are numbered counterclockwise with the face $F_{p}$ defined by the endpoints $\left(y_{2}^{(p, q)}, y_{3}^{(p, q)}\right)$ and $\left(y_{2}^{(p+1, q)}, y_{3}^{(p+1, q)}\right)$ for $p=1,2,3,4$ such that $p+1 \rightarrow p$ when $p=4$. The orientation of the face is defined by the unit normal $n^{(p, q)}=$ $\left[n_{2}^{(p, q)}, n_{3}^{(p, q)}\right]$ whose elements are defined by the vertex coordinates and the associated lengths.
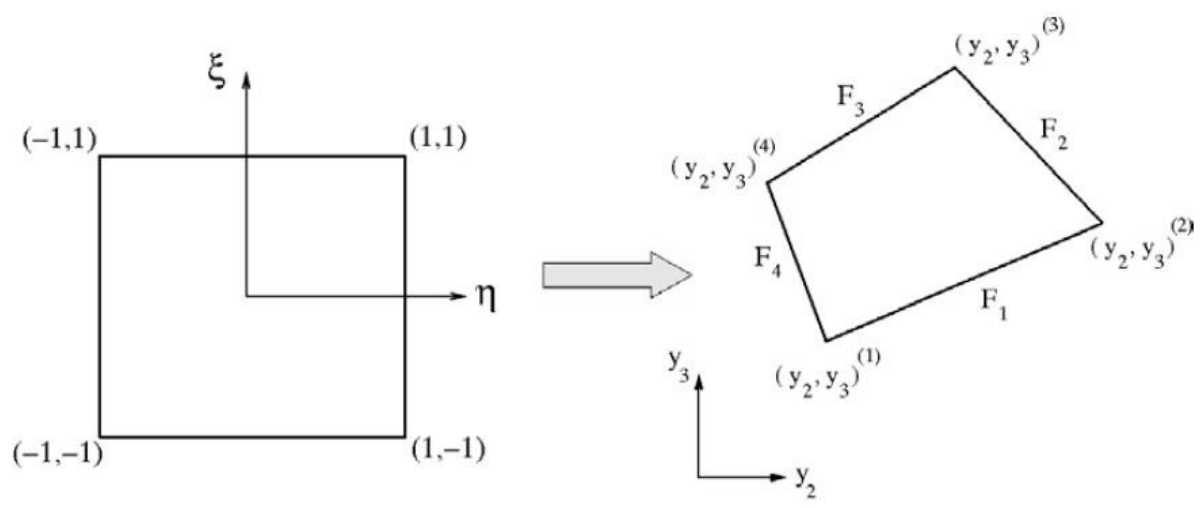

Figure 2.1 A reference square subvolume in the $\eta-\xi$ plane (left) mapped onto a quadrilateral subvolume in the $y_{2}-y_{3}$ plane (right) of the actual microstructure.

Following the 0th-order homogenization theory, (Bensoussan et al. 1978, Suquet 1987, Charalambakis 2010), the displacement field in the $q$ th subvolume is represented by the twoscale expansion involving macroscopic and fluctuating components

$$
u_{i}^{q}(\boldsymbol{x}, \boldsymbol{y}(\eta, \xi))=\bar{\varepsilon}_{i j} x_{j}+u_{i}^{\prime(q)}(\eta, \xi)
$$

The fluctuating displacements $u_{i}^{\prime(q)}(i=1,2,3)$ are approximated by the 2 nd order, Legendretype polynomial expansion in the reference coordinates $(\eta, \xi)$ consistent with the generalized plane strain constraint which ensures that $\bar{\varepsilon}_{11}=\bar{\varepsilon}_{11}^{(q)}$ for all subvolumes under all loading conditions,

$$
\begin{gathered}
u_{i}^{\prime(q)}=W_{i(00)}^{(q)}+\eta W_{i(10)}^{(q)}+\xi W_{i(01)}^{(q)}+\frac{1}{2}\left(3 \eta^{2}-1\right) W_{i(20)}^{(q)}+ \\
\frac{1}{2}\left(3 \xi^{2}-1\right) W_{i(02)}^{(q)}
\end{gathered}
$$

The unknown coefficients $W_{i(m n)}^{(q)}$ are ultimately expressed in terms of the surface-averaged displacements.

The local strains are then obtained in terms of the macroscopic and fluctuating strain components upon use of the strain-displacement relations 


$$
\varepsilon_{i j}^{(q)}=\bar{\varepsilon}_{i j}+\varepsilon_{i j}^{\prime(q)}=\bar{\varepsilon}_{i j}+\frac{1}{2}\left(\frac{\partial u_{i}^{\prime(q)}}{\partial y_{j}}+\frac{\partial u_{j}^{\prime(q)}}{\partial y_{i}}\right)
$$

Subsequently, a local stiffness matrix for the $q$ th subvolume is constructed by relating the surface-averaged fluctuating displacements to the surface-averaged tractions on each face of the subvolume. Towards this end, we express the 15 unknown coefficients $W_{i(m n)}^{(q)}$ in the displacement field representation in terms of the surface-averaged displacements. The definitions of the surface-averaged displacements provide 12 relations between the surface-averaged displacements and the unknown coefficients. These can be compactly expressed in terms of equations relating the 1 st and 2 nd order coefficients, $W_{i(10)}^{(q)}, W_{i(01)}^{(q)}$ and $W_{i(20)}^{(q)}, W_{i(02)}^{(q)}$ to the surface-averaged displacements and the 0 th order coefficients $W_{i(00)}^{(q)}$. The remaining three relations are obtained from the three equilibrium equations satisfied in the large (volumeaveraged sense or surface-averaged sense through the Gauss theorem) for each subvolume. The integration of interfacial displacements is done in the $\eta-\xi$ plane, while interfacial tractions are integrated along quadrilateral subvolume faces in the actual microstructure using the coordinate transformation described in the sequel. Following Achenbach (1975), the surface-averaged displacements on the $p$ th face of the $q$ th subvolume are defined by

$$
\hat{u}_{i}^{\prime(1,3)}=\frac{1}{2} \int_{-1}^{+1} u_{i}^{\prime}(\eta, \mp 1) d \eta, \quad \hat{u}_{i}^{\prime(2,4)}=\frac{1}{2} \int_{-1}^{+1} u_{i}^{\prime}( \pm 1, \xi) d \xi
$$

which are expressed in terms of the unknown 0 th, 1 st and 2 nd order $W_{i(m n)}^{(q)}$ coefficients upon integration. The superscripts $p=1,2,3,4$ indicate subvolume face number, Figure 2.1, omitting for clarity the superscripts $(q)$ which identify a given subvolume.

Similarly, the surface-averaged tractions are defined as follows

$$
\hat{t}_{i}^{\prime(1,3)}=\frac{1}{2} \int_{-1}^{+1} t_{i}(\eta, \mp 1) d \eta, \quad \hat{t}_{i}^{\prime(2,4)}=\frac{1}{2} \int_{-1}^{+1} t_{i}( \pm 1, \xi) d \xi
$$

where $t_{i}^{(p)}=\sigma_{j i}^{(p)} n_{j}^{(p)}$ from Cauchy's relations. The stresses associated with each surface are expressed in terms of the corresponding strains through Hooke's law for the $q$ th subvolume,

$$
\sigma_{i j}^{(q)}=C_{i j k l}^{(q)}\left(\varepsilon_{k l}^{(q)}-\varepsilon_{k l}^{t h(q)}\right)
$$

where $\varepsilon_{k l}^{t h(q)}=\alpha_{k l}^{(q)} \Delta T$ are the thermal strains and $\alpha_{k l}^{(q)}$ are the components of the thermal expansion tensor. The elastic subvolumes may be orthotropic or (transversely) isotropic. 
The surface-averaged strains on the faces of quadrilateral subvolumes in the actual microstructure needed in the expressions for the surface-averaged tractions are generated using the following relations between surface-averaged partial derivatives of the displacement field in the two coordinate systems

$$
\left[\begin{array}{l}
\frac{\partial \hat{u}_{l}^{\prime}}{\partial y_{2}} \\
\frac{\partial \bar{u}_{l}^{\prime}}{\partial y_{3}}
\end{array}\right]^{(p)}=\hat{\mathbf{J}}\left[\begin{array}{l}
\frac{\partial \hat{u}_{\imath}^{\prime}}{\partial \eta} \\
\frac{\partial \hat{u}_{l}^{\prime}}{\partial \xi}
\end{array}\right]^{(\hat{p})} \quad \text { where } \hat{\mathbf{J}}^{-1}=\overline{\mathbf{J}}=\frac{1}{4} \int_{-1}^{+1} \int_{-1}^{+1} J d \eta d \xi
$$

where $\mathbf{J}$ is the Jacobian of the transformation. The superscripts $p$ and $\hat{p}$ denote the faces of quadrilateral and reference subvolumes, respectively, with the following correspondence $\hat{p}=1,3 \rightarrow \xi=\mp 1$ and $\hat{p}=2,4 \rightarrow \eta= \pm 1$. The local stiffness matrix construction is simplified by approximating the relation between surface-averaged displacement gradients on the $p$ th face of the $q$ th subvolume in the reference and actual coordinate systems using volumeaveraged Jacobian $\overline{\mathbf{J}}$ in the above equation.

The surface-averaged strains are obtained in terms of the unknown coefficients in the subvolume displacement representation which are then expressed explicitly in terms of the surface-averaged fluctuating displacement components upon use of the definitions given in Eq. (2.5) and the application of equilibrium equations in the large (which provide the remaining three equations)

$$
\int_{S_{q}} \sigma \cdot n d S=\int_{S_{q}} t d S=\sum_{p=1}^{4} \int_{l_{p}} t^{(p)} d l_{p}=\sum_{p=1}^{4} l_{p} \hat{t}^{(p)}=0
$$

This leads to the relationship between the surface-averaged tractions and surface-averaged fluctuating displacements given in terms of the local stiffness matrix $\mathbf{K}^{(q)}$ for the $q$ th subvolume

$$
\hat{\mathbf{t}}^{(q)}=\mathbf{K}^{(q)} \widehat{\mathbf{u}}^{(q)}+\mathbf{N}^{(q)} \mathbf{C}^{(q)}\left(\overline{\boldsymbol{\varepsilon}}-\hat{\boldsymbol{\varepsilon}}^{t h(q)}\right)
$$

where $\hat{\mathbf{t}}=\left[\hat{\mathbf{t}}^{(1)} \hat{\mathbf{t}}^{(2)} \hat{\mathbf{t}}^{(3)} \hat{\mathbf{t}}^{(4)}\right]^{\mathrm{T}}$, and $\mathrm{N}=\left[\mathbf{n}^{(1)} \mathbf{n}^{(2)} \mathbf{n}^{(3)} \mathbf{n}^{(4)}\right]^{\mathrm{T}}$ contains unit vectors that define the orientation of each of the four subvolume faces. For subvolumes containing orthotropic, transversely isotropic or isotropic materials, the stress-stain relations are separated into out-ofplane shear and in-plane normal and shear contributions, and consequently the corresponding relations between surface-averaged tractions and surface-averaged fluctuating displacements are separated. The local stiffness matrices $\mathbf{K}^{(q)}$ for in-plane and out-of-plane loading share the same 
form of general expression, which is the product of the matrices $\overline{\mathbf{A}}$ and $\overline{\mathbf{B}}$ shown in the following with the omission of superscript $q$,

$$
\mathbf{K}=\overline{\mathbf{A}} \overline{\mathbf{B}} \text {, with } \overline{\mathbf{A}}=\mathbf{D C E B A}, \overline{\mathbf{B}}=\overline{\mathbf{P}}-\mathbf{N} \boldsymbol{\Phi}^{-\mathbf{1}} \mathbf{\Theta M}
$$

where $\mathbf{D}$ is an assembly of normal vectors associated with subvolume faces, $\mathbf{C}$ is the matrix of material properties, $\mathbf{B}$ is an assembly of Jacobian matrices arranged diagonally, A contains information on subvolume vertices, $\boldsymbol{\Phi}$ and $\boldsymbol{\Theta}$ contain information on material properties and Jacobian matrix, E, $\overline{\mathbf{P}}, \mathbf{N}, \mathbf{M}$ are supplementary matrices containing only 0 and 1 elements, (Khatam and Pindera 2009a).

For both in-plane and out-of-plane problems, the relationships between surface-averaged tractions and surface-averaged fluctuating displacements have the same form, Eq. (2.10). The local stiffness matrix $\mathbf{K}^{(q)}$ is comprised of $4 \times 4$ submatrices $\mathbf{K}_{i j}^{(q)}$ as shown below

$$
\mathbf{K}^{(q)}=\left[\begin{array}{llll}
\mathbf{K}_{11} & \mathbf{K}_{12} & \mathbf{K}_{13} & \mathbf{K}_{14} \\
\mathbf{K}_{21} & \mathbf{K}_{22} & \mathbf{K}_{23} & \mathbf{K}_{24} \\
\mathbf{K}_{31} & \mathbf{K}_{32} & \mathbf{K}_{33} & \mathbf{K}_{34} \\
\mathbf{K}_{41} & \mathbf{K}_{42} & \mathbf{K}_{43} & \mathbf{K}_{44}
\end{array}\right]^{(q)}
$$

For the in-plane traction components, a submatrix $\mathbf{K}_{i j}^{(q)}$ contains $2 \times 2$ elements, for instance, $\mathbf{K}_{11}^{(q)}=\left[\begin{array}{ll}k_{11} & k_{12} \\ k_{21} & k_{22}\end{array}\right]^{(q)}$. For the out-of-plane traction components, $\mathbf{K}_{i j}^{(q)}$ contains a single element, for instance, $\mathbf{K}_{11}^{q}=\left[k_{11}^{o p}\right]^{(q)}$. Eqs. (2.12-2.13) below illustrate the closed-form expressions for the elements $k_{11}$ and $k_{11}^{o p}$ of the for $q$ th subvolume under in-plane and out-of-plane loading, respectively.

$$
\begin{gathered}
k_{11}=-\frac{1}{2} n_{2}^{(1)} C_{22} \hat{J}_{23}-\frac{1}{2} n_{3}^{(1)} C_{44} \hat{J}_{33} \\
-3\left(n_{2}^{(1)} C_{22} \hat{J}_{23}+n_{3}^{(1)} C_{44} \hat{J}_{33}\right)\left(\frac{1}{2}-\Phi_{11}^{-1} \Theta_{12}-\Phi_{12}^{-1} \Theta_{22}\right) \\
+3\left(n_{3}^{(1)} C_{44} \hat{J}_{23}+n_{2}^{(1)} C_{23} \hat{J}_{33}\right)\left(\Phi_{21}^{-1} \Theta_{12}-\Phi_{22}^{-1} \Theta_{22}\right) \\
k_{11}^{o p}=-\frac{1}{2} n_{2}^{(1)} C_{66} \hat{J}_{23}-\frac{1}{2} n_{3}^{(1)} C_{55} \hat{J}_{33}-3\left(n_{2}^{(1)} C_{66} \hat{J}_{23}+n_{3}^{(1)} C_{55} \hat{J}_{33}\right)\left(\frac{1}{2}-\frac{\Theta_{12}^{o p}}{\Phi^{o p}}\right)
\end{gathered}
$$

where $\mathrm{n}$ is the normal vector to subvolume face, and $\hat{J}$ is volume-averaged Jacobian matrix. 


\subsection{Traction and Displacement Continuity at Subvolume Interfaces}

The unknown interfacial surface-average displacements are determined by solving a global system of equations generated by first enforcing traction continuity at each interface between two adjacent subvolumes, followed by direct enforcement of displacement continuity. In the present FVDAM version, row-wise and column-wise unit cell discretization is employed. Hence, proceeding from left to right, row-wise enforcement of traction continuity between adjacent subvolumes takes the form

$$
\hat{\mathbf{t}}^{(2, q-1)}+\hat{\mathbf{t}}^{(4, q)}=0
$$

Similarly, proceeding in the upward direction, column-wise enforcement of displacement continuity between adjacent subvolumes takes the form

$$
\hat{\mathbf{t}}^{(3, \bar{q}-1)}+\hat{\mathbf{t}}^{(1, \bar{q})}=0
$$

In the above equations, the superscripts $q-1, q$ and $\bar{q}-1, \bar{q}$ are associated with adjacent subvolumes along rows and columns, respectively. The traction continuity equations are then expressed in terms of surface-averaged displacements, applied macroscopic and thermal strains using the local stiffness matrix relations given by Eq. (2.10). In the case of intact or continuous interfaces considered in numerous previous FVDAM investigations, the displacement continuity is satisfied directly by setting equal the interfacial displacements at the common faces of adjacent subvolumes.

In the case of interfaces that undergo separation considered herein, we introduce displacement discontinuity functions as follows. Proceeding from left to right and then upward, we define

$$
\begin{aligned}
& \widehat{\mathbf{u}}^{\prime(4, q)}-\widehat{\mathbf{u}}^{\prime(2, q-1)}=\widehat{\mathbf{u}}_{*}^{\prime(4, q)} \\
& \widehat{\mathbf{u}}^{\prime(1, \bar{q})}-\widehat{\mathbf{u}}^{\prime(3, \bar{q}-1)}=\widehat{\mathbf{u}}_{*}^{\prime(1, \bar{q})}
\end{aligned}
$$

Following Chen and Pindera (2007a), the interfacial displacements are then expressed in terms of continuous and discontinuous contributions. For the surface-averaged displacements between adjacent subvolumes along the rows, we have,

$$
\begin{aligned}
& \widehat{\mathbf{u}}^{\prime(2, q-1)}=\widehat{\mathbf{u}}_{o}^{(4, q)}-\left[\mathbf{K}^{*, q}\right]^{-1} \mathbf{K}_{44}^{(q)} \widehat{\mathbf{u}}_{*}^{(4, q)} \\
& \widehat{\mathbf{u}}^{\prime(4, q)}=\widehat{\mathbf{u}}_{o}^{(4, q)}+\left[\mathbf{K}^{*, q}\right]^{-1} \mathbf{K}_{22}^{(q-1)} \widehat{\mathbf{u}}_{*}^{(4, q)}
\end{aligned}
$$

where $\left[\mathbf{K}^{*, q}\right]^{-1}=\left[\mathbf{K}_{22}^{(q-1)}+\mathbf{K}_{44}^{(q)}\right]^{-1}$, and along the columns 


$$
\begin{aligned}
& \widehat{\mathbf{u}}^{\prime(3, \bar{q}-1)}=\widehat{\mathbf{u}}_{o}^{(1, \bar{q})}-\left[\mathbf{K}^{*, \bar{q}}\right]^{-1} \mathbf{K}_{11}^{(\bar{q})} \widehat{\mathbf{u}}_{*}^{(1, \bar{q})} \\
& \widehat{\mathbf{u}}^{\prime(1, \bar{q})}=\widehat{\mathbf{u}}_{o}^{(1, \bar{q})}+\left[\mathbf{K}^{*, \bar{q}}\right]^{-1} \mathbf{K}_{33}^{(\bar{q}-1)} \widehat{\mathbf{u}}_{*}^{(1, \bar{q})}
\end{aligned}
$$

where $\left[\mathbf{K}^{*, \bar{q}}\right]^{-1}=\left[\mathbf{K}_{33}^{(\bar{q}-1)}+\mathbf{K}_{11}^{(\bar{q})}\right]^{-1}$, Figure 2.2. For intact interfaces, we recover standard interfacial displacement continuity conditions: $\widehat{\mathbf{u}}^{(2, q-1)}=\widehat{\mathbf{u}}^{\prime(4, q)}=\widehat{\mathbf{u}}_{o}^{\prime(4, q)}$ and $\widehat{\mathbf{u}}^{(3, \bar{q}-1)}=$ $\widehat{\mathbf{u}}^{\prime(1, \bar{q})}=\widehat{\mathbf{u}}_{o}^{(1, \bar{q})}$.

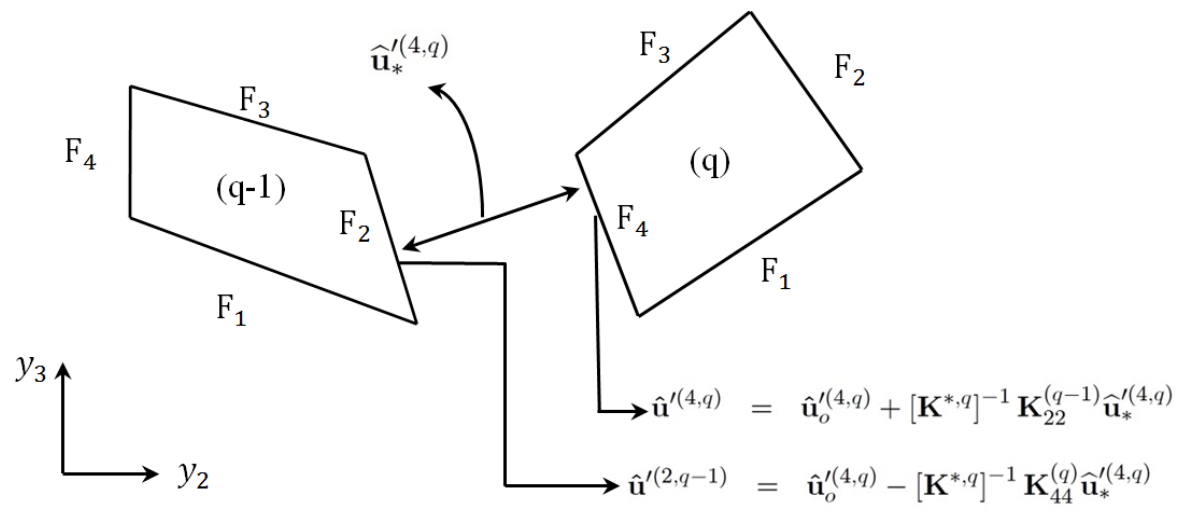

Figure 2.2 Interfacial discontinuity between two adjacent subvolumes.

Using the above surface-averaged displacement representations in the presence of interfacial separation, and assuming isolated interfaces that undergo separation, the traction continuity relations given by Eqs. (2.14-2.15) become,

$$
\begin{aligned}
\mathbf{K}_{24}^{(q-1)} \widehat{\mathbf{u}}_{o}^{\prime(4, q-1)}+\left(\mathbf{K}_{22}^{(q-1)}+\mathbf{K}_{44}^{(q)}\right) \widehat{\mathbf{u}}_{o}^{\prime(4, q)}+\mathbf{K}_{42}^{(q)} \widehat{\mathbf{u}}_{o}^{\prime(4, q+1)}+\mathbf{K}_{21}^{(q-1)} \widehat{\mathbf{u}}^{\prime(1, q-1)} \\
+\mathbf{K}_{23}^{(q-1)} \widehat{\mathbf{u}}^{\prime(3, q-1)}+\mathbf{K}_{41}^{(q)} \widehat{\mathbf{u}}^{\prime(1, q)}+\mathbf{K}_{43}^{(q)} \widehat{\mathbf{u}}^{\prime(3, q)} \\
+\left(\overline{\mathbf{C}}^{(q-1)}+\overline{\mathbf{C}}^{(q)}\right)\left(\overline{\boldsymbol{\varepsilon}}-\widehat{\boldsymbol{\varepsilon}}^{t h(q)}\right)=-\mathbf{L}^{(4, q)} \widehat{\mathbf{u}}_{*}^{\prime(4, q)}
\end{aligned}
$$

and

$$
\begin{aligned}
\mathbf{K}_{31}^{(\bar{q}-1)} \widehat{\mathbf{u}}_{o}^{(1, \bar{q}-1)}+\left(\mathbf{K}_{33}^{(\bar{q}-1)}+\mathbf{K}_{11}^{(\bar{q})}\right) \widehat{\mathbf{u}}_{o}^{\prime(1, \bar{q})}+\mathbf{K}_{13}^{(\bar{q})} \widehat{\mathbf{u}}_{o}^{\prime(1, \bar{q}+1)}+\mathbf{K}_{32}^{(\bar{q}-1)} \widehat{\mathbf{u}}^{\prime(2, \bar{q}-1)} \\
+\mathbf{K}_{34}^{(\bar{q}-1)} \widehat{\mathbf{u}}^{\prime(4, \bar{q}-1)}+\mathbf{K}_{12}^{(\bar{q})} \widehat{\mathbf{u}}^{\prime(2, \bar{q})}+\mathbf{K}_{14}^{(\bar{q})} \widehat{\mathbf{u}}^{\prime(4, \bar{q})} \\
+\left(\overline{\mathbf{C}}^{(\bar{q}-1)}+\overline{\mathbf{C}}^{(q)}\right)\left(\overline{\boldsymbol{\varepsilon}}-\widehat{\boldsymbol{\varepsilon}}^{t h(q)}\right)=-\mathbf{L}^{(1, \bar{q})} \widehat{\mathbf{u}}_{*}^{\prime(1, \bar{q})}
\end{aligned}
$$

where

$$
\begin{aligned}
& \mathbf{L}^{(4, q)}=\left(\mathbf{K}_{44}^{(q)}\left[\mathbf{K}^{*, q}\right]^{-1} \mathbf{K}_{22}^{(q-1)}-\mathbf{K}_{22}^{(q-1)}\left[\mathbf{K}^{*, q}\right]^{-1} \mathbf{K}_{44}^{(q)}\right) \\
& \mathbf{L}^{(1, \bar{q})}=\left(\mathbf{K}_{11}^{(\bar{q})}\left[\mathbf{K}^{*, \bar{q}}\right]^{-1} \mathbf{K}_{33}^{(\bar{q}-1)}-\mathbf{K}_{33}^{(\bar{q}-1)}\left[\mathbf{K}^{*, \bar{q}}\right]^{-1} \mathbf{K}_{11}^{(\bar{q})}\right)
\end{aligned}
$$


In the presence of additional separations along the remaining interfaces of the adjacent subvolumes $(q-1, q)$ and $(\bar{q}-1, \bar{q})$ the above equations are modified accordingly.

\subsection{Periodic Boundary Conditions}

In the extended FVDAM theory, interfaces undergoing damage are not allowed to be inserted along the unit cell boundaries. Hence the same manner of applying periodic boundary conditions used by Khatam and Pindera (2009a, b), Bansal and Pindera (2006) is employed. Figure 2.3 illustrates the enforcement of periodic boundary conditions for a square unit cell with row-wise and column-wise discretization. Along row-wise direction the tractions along left face (face 2) of the 1st subvolume are balanced by the tractions along right face (face 4) of the $q$ th subvolume and the displacements along left face of the 1st subvolume are set equal to the displacements along right face of the $q$ th subvolume. Similarly, along column-wise direction the tractions along bottom face (face 1) of the $\overline{1}$ st subvolume are balanced by the tractions along top face (face 3 ) of the $\bar{q}$ th subvolume and the displacements along bottom face of the $\overline{1}$ st subvolume are set equal to the displacement along top face of the $\bar{q}$ th subvolume.

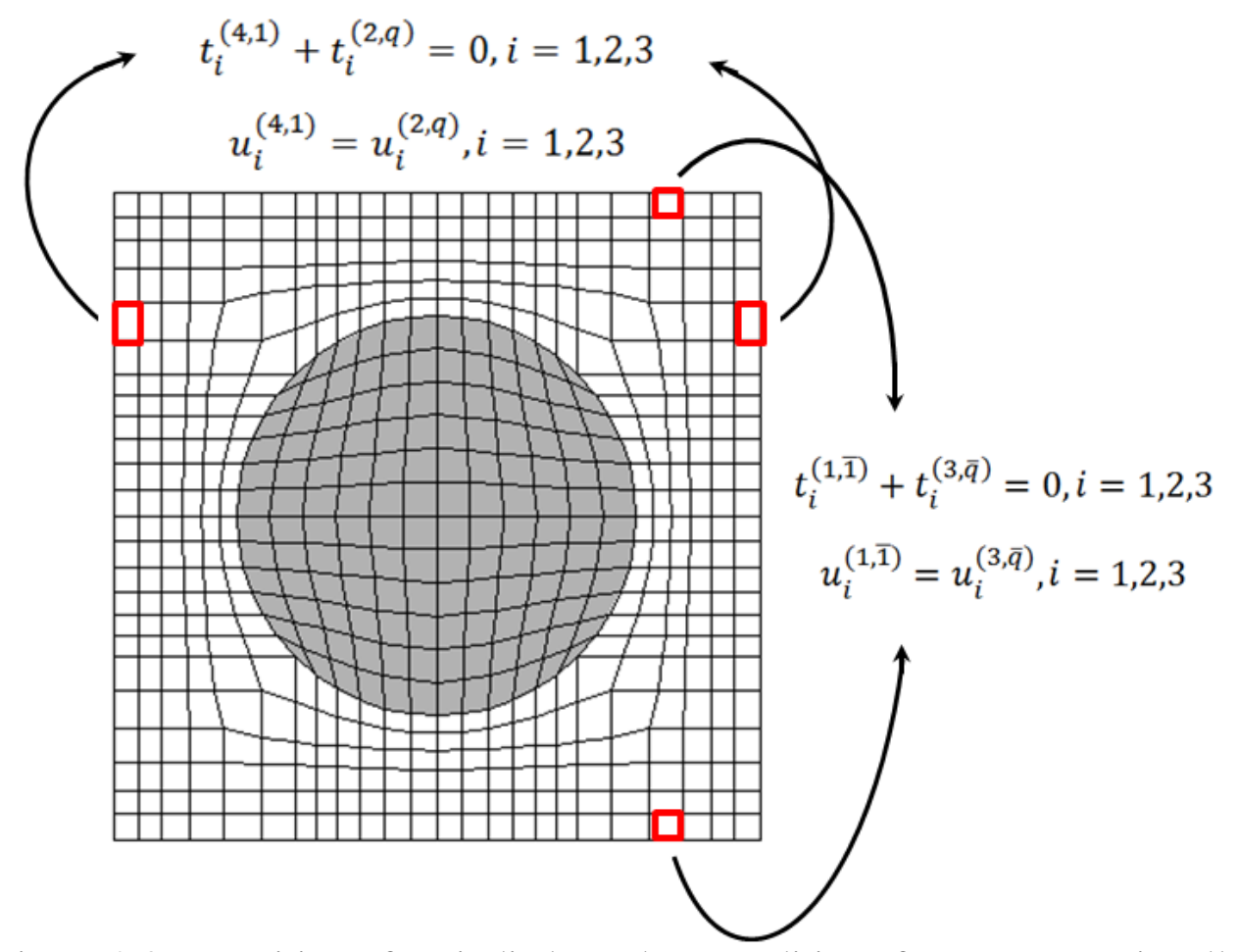

Figure 2.3 Imposition of periodic boundary conditions for a square unit cell. 


\subsection{Primary and Auxiliary Global Systems of Equations}

As shown in Eqs. (2.19-2.20), imposition of interfacial traction and displacement continuity conditions at the common subvolume faces, together with periodic boundary conditions, produces the global system of equations containing the common surface-averaged fluctuating displacements and the displacement discontinuities that symbolically takes the form,

$$
\mathbb{k} \widehat{\mathbf{U}}_{o}^{\prime}=\Delta \mathbf{C} \overline{\boldsymbol{\varepsilon}}+\Delta \mathbf{\Gamma} \Delta T-\mathbb{L} \widehat{\mathbf{U}}_{*}^{\prime}
$$

where $\mathbb{k}$ is the global stiffness matrix containing information on the subvolume geometry and material assignment, the matrix $\Delta \mathbf{C}$ is comprised of the differences in the material stiffness matrices of adjacent subvolumes, and $\mathbb{L}$ is the matrix that represents contributions from damaged or cracked interfaces. This is the primary system of equations that is used to express the continuous surface-averaged fluctuating interfacial displacements $\widehat{\mathbf{U}}_{o}^{\prime}$ in terms of the corresponding displacement discontinuities $\widehat{\mathbf{U}}_{*}^{\prime}$. The additional or auxiliary equations necessary for the determination of the displacement discontinuities come from the traction conditions applied to the damaged interfaces described next.

The interfacial tractions are related to the fluctuating surface-averaged common displacements and the corresponding displacement discontinuities in the presence of interfacial damage via the local stiffness matrix expression given in Eq. (2.10). In the latter case, tractions may be specified directly, e.g., using traction-free crack-face condition or via a chosen tractioninterfacial separation relation. In the presence of multiple interfaces undergoing damage, the local stiffness matrix relationship for a particular interface involves displacement discontinuities associated with the faces of the particular subvolume as well as displacement discontinuities associated with other interfaces. This coupling comes through the adjacent continuous surfaceaveraged interfacial displacements obtained from the solution of the primary system of equations. The auxiliary equations for the tractions along the interfaces undergoing damage may be symbolically expressed as follows

$$
\mathbf{T}=\mathbf{K}_{*} \widehat{\mathbf{U}}_{*}^{\prime}+\mathbf{K}_{o} \widehat{\mathbf{U}}_{o}^{\prime}+\mathbf{C} \overline{\boldsymbol{\varepsilon}}+\mathbf{\Gamma} \Delta T
$$

where the matrix $\mathbf{K}_{*}$ contains the elements associated with displacement discontinuities, which are products of the local stiffness matrices $\mathbf{K}^{(q)}$ and elements of the matrices appearing in Eqs. (2.17-2.18), $\mathbf{K}_{o}$ contains the local stiffness matrices associated with the undamaged interfaces, and $\mathbf{C} \overline{\boldsymbol{\varepsilon}}$ and $\boldsymbol{\Gamma} \Delta T$ are the assembled loading vectors. Eq. (2.23) shows the detailed expressions 
appearing in the auxiliary equations extracted from the left and bottom subvolume faces for two cases with interfaces inserted between adjacent subvolumes $(q-1, q)$ and $(\bar{q}-1, \bar{q})$, respectively, with the corresponding primary system of equations shown in Eqs. (2.16-2.17). It should be noted that displacement continuity conditions were employed in those two equations along row-wise and column-wise directions, respectively.

$$
\begin{aligned}
& \hat{\mathbf{t}}^{(4, q)}= \mathbf{K}_{44}^{(q)}\left[\mathbf{K}^{*, q}\right]^{-1} \mathbf{K}_{22}^{(q-1)} \widehat{\mathbf{u}}_{*}^{\prime(4, q)}+\mathbf{K}_{44}^{(q)} \widehat{\mathbf{u}}_{0}^{\prime(4, q)}+\mathbf{K}_{42}^{(q)} \widehat{\mathbf{u}}_{o}^{\prime(4, q+1)}+\mathbf{K}_{41}^{(q)} \widehat{\mathbf{u}}^{\prime(1, q)} \\
&+\mathbf{K}_{43}^{(q)} \widehat{\mathbf{u}}^{\prime(3, q)}+\overline{\mathbf{C}}^{(q)}\left(\overline{\boldsymbol{\varepsilon}}-\widehat{\boldsymbol{\varepsilon}}^{t h(q)}\right) \\
& \hat{\mathbf{t}}^{(1, \bar{q})=} \mathbf{K}_{11}^{(\bar{q})}\left[\mathbf{K}^{*, \bar{q}}\right]^{-1} \mathbf{K}_{33}^{(\bar{q}-1)} \widehat{\mathbf{u}}_{*}^{\prime(1, \bar{q})}+\mathbf{K}_{11}^{(\bar{q})} \widehat{\mathbf{u}}_{o}^{(1, \bar{q})}+\mathbf{K}_{13}^{(\bar{q})} \widehat{\mathbf{u}}_{o}^{\prime(1, \bar{q}+1)}+\mathbf{K}_{12}^{(\bar{q})} \widehat{\mathbf{u}}^{\prime(2, \bar{q})} \\
&+\mathbf{K}_{14}^{(\bar{q})} \widehat{\mathbf{u}}^{\prime(4, \bar{q})}+\overline{\mathbf{C}}^{(\bar{q})}\left(\overline{\boldsymbol{\varepsilon}}-\widehat{\boldsymbol{\varepsilon}}^{t h(\bar{q})}\right)
\end{aligned}
$$

From the primary system of equations we then obtain the continuous fluctuating surfaceaveraged interfacial displacements in terms of displacement discontinuities,

$$
\widehat{\mathbf{U}}_{o}^{\prime}=\mathbb{k}^{-1}\left(\Delta \mathbf{C} \overline{\boldsymbol{\varepsilon}}+\Delta \boldsymbol{\Gamma} \Delta T-\mathbb{L} \widehat{\mathbf{U}}_{*}^{\prime}\right)
$$

Substituting the expression for $\widehat{\mathbf{U}}_{o}^{\prime}$ into the auxiliary system of equations, we finally obtain the auxiliary system of equations that relates the tractions of damaged interfaces to the corresponding displacement discontinuities and the applied loading,

$$
\mathbf{T}\left(\widehat{\mathbf{U}}_{*}^{\prime}\right)=\left(\mathbf{K}_{*}-\mathbf{K}_{o} \mathbb{k}^{-1} \mathbb{L}\right) \widehat{\mathbf{U}}_{*}^{\prime}+\mathbf{K}_{o} \mathbb{k}^{-1} \Delta \mathbf{C} \overline{\boldsymbol{\varepsilon}}+\mathbf{K}_{o} \mathbb{k}^{-1} \Delta \mathbf{\Gamma} \Delta T+\mathbf{C} \overline{\boldsymbol{\varepsilon}}+\mathbf{\Gamma} \Delta T
$$

The tractions appearing on the right hand side of the auxiliary system of equations may be

specified directly or through a chosen traction-displacement discontinuity relation $\mathbf{T}\left(\widehat{\mathbf{U}}_{*}^{\prime}\right)$. In the case of a nonlinear traction-displacement discontinuity relation, an incremental and iterative (Newton-Raphson) procedure is applied to solve the auxiliary system of equations.

\subsection{Cohesive Zone Model Implementation}

Herein, for in-plane normal and shear problems we incorporate the coupled bilinear tractionseparation relations, whereas for out-of-plane shear problems the bilinear traction-separation relation is incorporated assuming no coupling between in-plane and out-of-plane tractionseparation relations. In the following, the procedures to incorporate the coupled bilinear traction-separation relation are discussed in detail, while the procedure to incorporate the uncoupled bilinear traction-separation relation is straightforward and is described briefly. 
The coupled bilinear traction-separation relations incorporated herein are frequently employed in finite-element CZM-based damage evolution studies, (Geubelle and Baylor 1998, Chandra et al. 2002, Matous and Geubelle 2006, Song et al. 2006). The non-dimensional effective separation displacement defined by

$$
\lambda_{e}=\sqrt{\left(\frac{\delta_{n}}{\Delta_{n}^{c}}\right)^{2}+\left(\frac{\delta_{t}}{\Delta_{t}^{c}}\right)^{2}}
$$

is employed to couple the normal traction-displacement discontinuity and shear tractiondisplacement discontinuity relations. In the above definition, $\delta_{n}$ and $\delta_{t}$ are the normal and tangential displacement discontinuities, and $\Delta_{n}^{c}$ and $\Delta_{t}^{c}$ are the corresponding critical values at complete separation. Following common practice, we set the critical values equal, $\Delta_{n}^{c}=\Delta_{n}^{c}=$ $\Delta^{c}$. The maximum non-dimensional displacement $\lambda_{\max }$ which corresponds to the maximum tractions is incorporated to adjust the elastic stiffness by adjusting the pre-peak slope of the traction-separation relation. With the above defined quantities, the bilinear interfacial separation relation takes the following forms for the respective loading cases when the interface is under tension:

For $\delta_{n}>0$ and $\lambda_{e}<\lambda_{\max }$

$$
t_{n}=\sigma_{\max } \frac{1}{\lambda_{\max }}\left(\frac{\delta_{n}}{\Delta^{c}}\right), \quad t_{t}=\sigma_{\max } \frac{1}{\lambda_{\max }}\left(\frac{\delta_{t}}{\Delta^{c}}\right)
$$

For $\delta_{n}>0$ and $\lambda_{e}>\lambda_{\max }$

$$
t_{n}=\sigma_{\max } \frac{1-\lambda_{e}}{1-\lambda_{\max }} \frac{1}{\lambda_{e}}\left(\frac{\delta_{n}}{\Delta^{c}}\right), \quad t_{t}=\sigma_{\max } \frac{1-\lambda_{e}}{1-\lambda_{\max }} \frac{1}{\lambda_{e}}\left(\frac{\delta_{t}}{\Delta^{c}}\right)
$$

when the interface is under compression, $\delta_{n}=0$ and only the shear traction relations hold in the above.

In order to implement an iterative Newton-Raphson technique in the solution of Eq. (2.25), the Jacobian of the stiffness matrix obtained from the gradient form of the tractioninterfacial separation relations is required,

$$
\left[\begin{array}{l}
d t_{n} \\
d t_{t}
\end{array}\right]=\left[\begin{array}{ll}
\frac{\partial t_{n}}{\partial \delta_{n}} & \frac{\partial t_{n}}{\partial \delta_{t}} \\
\frac{\partial t_{t}}{\partial \delta_{n}} & \frac{\partial t_{t}}{\partial \delta_{t}}
\end{array}\right]\left[\begin{array}{l}
d \delta_{n} \\
d \delta_{t}
\end{array}\right]=\left[\begin{array}{ll}
C_{n n} & C_{n t} \\
C_{t n} & C_{t t}
\end{array}\right]\left[\begin{array}{l}
d \delta_{n} \\
d \delta_{t}
\end{array}\right]
$$


For $\lambda_{e}<\lambda_{\max }$

$$
\begin{gathered}
C_{n n}=\frac{\partial t_{n}}{\partial \delta_{n}}=\frac{\sigma_{\max }}{\lambda_{\max } \Delta^{c}} \\
C_{n n}=C_{t n}=0 \\
C_{t t}=\frac{\partial t_{t}}{\partial \delta_{t}}=\frac{\sigma_{\max }}{\lambda_{\max } \Delta^{c}}
\end{gathered}
$$

For $\lambda_{e}>\lambda_{\max }$

$$
\begin{gathered}
C_{n n}=\frac{\partial t_{n}}{\partial \delta_{n}}=\frac{\sigma_{\max }}{\left(1-\lambda_{\max }\right) \Delta^{c}}\left(\frac{1}{\lambda_{e}}-\frac{\left(\delta_{n}\right)^{2}}{\left(\Delta^{c}\right)^{2}} \frac{1}{\lambda_{e}^{3}}-1\right) \\
C_{n t}=\frac{\partial t_{n}}{\partial \delta_{t}}=-\frac{\sigma_{\max }}{\left(1-\lambda_{\max }\right)} \frac{\delta_{n} \delta_{t}}{\left(\Delta^{c}\right)^{3}} \frac{1}{\lambda_{e}^{3}} \\
C_{t n}=\frac{\partial t_{t}}{\partial \delta_{n}}=-\frac{\sigma_{\max }}{\left(1-\lambda_{\max }\right)} \frac{\delta_{t} \delta_{n}}{\left(\Delta^{c}\right)^{3}} \frac{1}{\lambda_{e}^{3}} \\
C_{t t}=\frac{\partial t_{t}}{\partial \delta_{t}}=\frac{\sigma_{\max }}{\left(1-\lambda_{\max }\right) \Delta^{c}}\left(\frac{1}{\lambda_{e}}-\frac{\left(\delta_{t}\right)^{2}}{\left(\Delta^{c}\right)^{2}} \frac{1}{\lambda_{e}^{3}}-1\right)
\end{gathered}
$$

Figure 2.4 illustrates graphically the coupled normal and tangential interfacial separation laws and the resulting interaction effects. The coupling between the normal and tangential modes may be eliminated by eliminating coupling in the expression for the non-dimensionalized effective separation displacement. In this case, strictly bilinear traction-interfacial separation relations for each mode are obtained which are also included in Figure 2.4. These correspond to the planes $\delta_{t}=0$ and $\delta_{n}=0$ in the normal and tangential separation laws, respectively. For outof-plane shear loading, the separation law is the same as the in-plane bilinear traction-interfacial separation relation in tangential direction, Figure 2.4(b). 

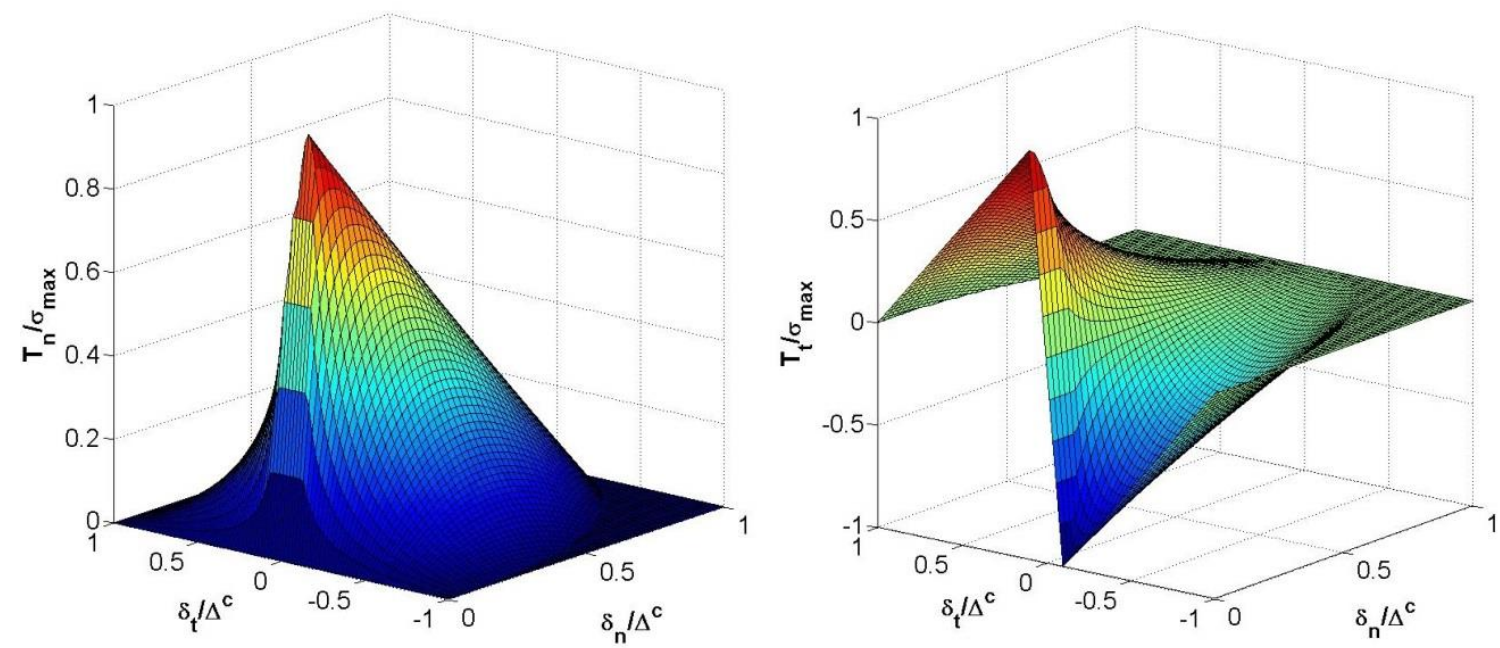

(a) Coupled separation laws
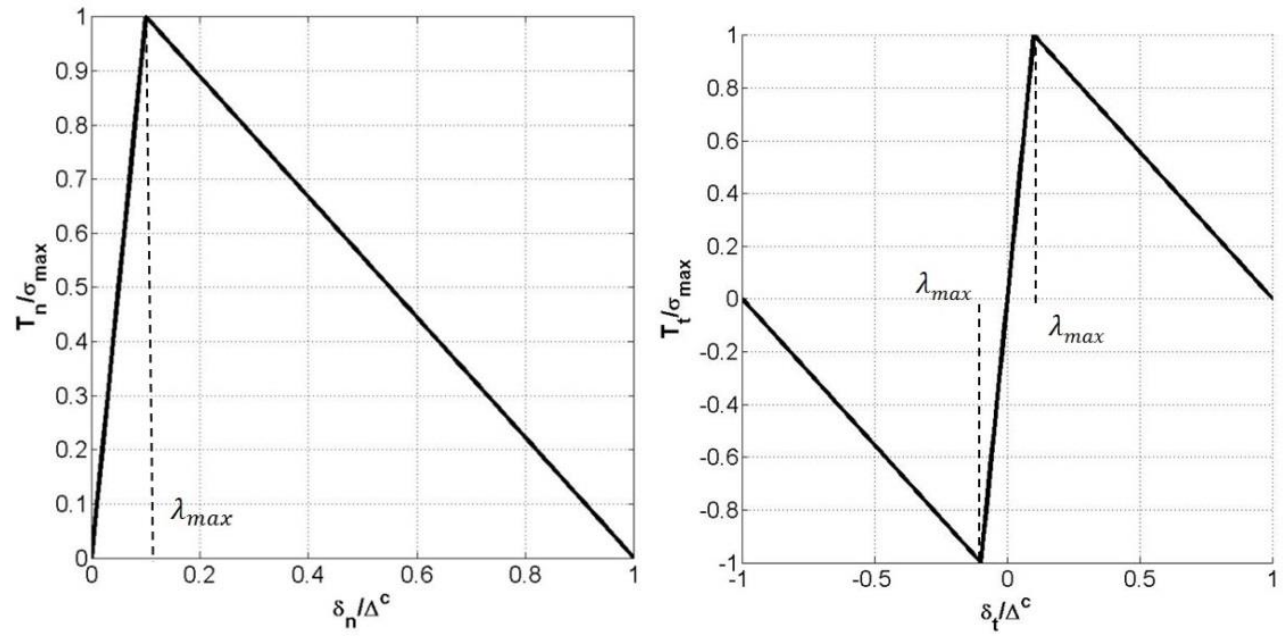

(b) Uncoupled separation laws

Figure 2.4 Traction-interfacial separation relations for the cohesive zone model in normal (left column) and tangential (right column) directions to the interface: graphical representations of (a) coupled and (b) uncoupled relations.

The interfacial separation laws are given in terms of normal and tangential displacement discontinuities, whereas the displacement discontinuities defined within the FVDAM framework, Eqs. (2.17-2.18), are referred to the Cartesian coordinate system associated with the unit cell. Hence, in order to implement the above separation laws into the FVDAM framework the traction and displacement discontinuities appearing in the auxiliary global equations, Eqs. (2.25), are transformed to local coordinate systems that are normal and tangential to the subvolume faces undergoing separation. When the normal traction is compressive for the particular interface, the 
corresponding equation in the transformed system of auxiliary equations is simply eliminated and the standard traction and displacement continuity conditions, expressed through the common surface-averaged displacements appearing in the primary system of equations, suffice. This straight forward approach of implementing CZM into our finite-volume framework, enabled by partitioning the surface-averaged interfacial displacements using Eqs. (2.17-2.18), contrasts with the corresponding finite-element based implementation where large interfacial stiffness is employed in the interfacial traction-separation law in compression, potentially leading to material interpenetration. This significant difference will be discussed, and its effects illustrated, in more depth in Chapter 3.

\subsection{Homogenization}

Solution of the auxiliary system of equations for the surface-averaged displacement discontinuities $\widehat{\mathbf{U}}_{*}^{\prime}$, followed by the determination of the continuous surface-averaged fluctuating displacements $\widehat{\mathbf{U}}_{o}^{\prime}$ enables determination of the localization relations for the $q$ th subvolume

$$
\overline{\boldsymbol{\varepsilon}}^{(q)}=\mathbb{A}^{(q)} \overline{\boldsymbol{\varepsilon}}+\mathbb{D}^{(q)}
$$

where $\mathbb{A}^{(q)}$ are Hill's strain concentration matrices, Hill (1963). Application of one non-zero macroscopic strain component in the absence of damage determines one column vector of $\mathbb{A}^{(q)}$, which is done just once. On the other hand, the vector $\mathbb{D}^{(q)}$ which contains thermal and displacement discontinuity contributions to the $q$ th subvolume average strain is obtained at each increment of the applied macroscopic strains $\overline{\boldsymbol{\varepsilon}}$ upon solution of the unit cell boundary-value problem which generates $\overline{\boldsymbol{\varepsilon}}^{(q)}$. Hence, $\mathbb{D}^{(q)}=\overline{\boldsymbol{\varepsilon}}^{(q)}-\mathbb{A}^{(q)} \overline{\boldsymbol{\varepsilon}}$. Use of localization relations in the average composite stress definition, in conjunction with the volume-averaged stress strain relations for each subvolume, yields the homogenized or macroscopic constitutive equation for a multiphase composite in the presence of evolving interfacial damage,

$$
\overline{\boldsymbol{\sigma}}=\frac{1}{\mathbf{V}} \int_{V} \boldsymbol{\sigma}(x) d V=\sum_{q=1}^{N_{q}} v_{(q)} \overline{\boldsymbol{\sigma}}^{(q)}=\mathbf{C}^{*}\left[\overline{\boldsymbol{\varepsilon}}-\left(\overline{\boldsymbol{\varepsilon}}^{t h}+\overline{\boldsymbol{\varepsilon}}^{d}\right)\right]
$$

where $v_{(q)}=V^{q} / V$ is the volume fraction of the $q$ th subvolume. The homogenized stiffness matrix $\mathbf{C}^{*}$ and the thermal and damage strains are given in terms of the subvolume geometry, material properties, elastic concentration matrices and their thermo-damage counterparts 


$$
\begin{gathered}
\mathbf{C}^{*}=\sum_{q=1}^{N_{q}} v_{(q)} \mathbf{C}^{(q)} \mathbb{A}^{(q)} \\
\overline{\boldsymbol{\varepsilon}}^{t h}+\overline{\boldsymbol{\varepsilon}}^{d}=-\frac{\left[\mathbf{C}^{*}\right]^{-1}}{\mathrm{~V}} \sum_{q=1}^{N_{q}} v_{(q)}\left[\mathbf{C}^{(q)} \mathbb{D}^{(q)}-\boldsymbol{\Gamma}^{(q)} \Delta T\right]
\end{gathered}
$$

As observed in Eqs. (2.21-2.25), the solution of the unit cell boundary-value problem is obtained for any combination of specified macroscopic strains $\overline{\boldsymbol{\varepsilon}}$. In order to simulate loading by specified stress components, the strain components are adjusted accordingly using the homogenized constitutive equation $\overline{\boldsymbol{\sigma}}=\mathbf{C}^{*}\left(\left[\overline{\boldsymbol{\varepsilon}}-\left(\overline{\boldsymbol{\varepsilon}}^{t h}+\overline{\boldsymbol{\varepsilon}}^{d}\right)\right]\right)$. In the absence of damage and thermal loads, constant strain ratios that correspond to fixed stress ratios are obtained in terms of the homogenized moduli $\mathbf{C}^{*}$. In the presence of damage and thermal loads, the incremental version of the homogenized constitutive equations,

$$
d \overline{\boldsymbol{\sigma}}=\mathbf{C}^{*}\left(d \overline{\boldsymbol{\varepsilon}}-d \overline{\boldsymbol{\varepsilon}}^{d}-d \overline{\boldsymbol{\varepsilon}}^{t h}\right)
$$

is employed, and strain increment ratios are adjusted iteratively at each load increment to generate the desired load path specified in terms of macroscopic stresses.

The above CZM-based homogenization framework may be checked for consistency by comparing the applied macroscopic strains $\overline{\boldsymbol{\varepsilon}}$ with the homogenized strains obtained from the solution of the unit cell boundary-value problem at each load increment. Specifically, the average strain obtained from the unit cell surface displacement

$$
\bar{\varepsilon}_{i j}=\frac{1}{\mathbf{V}} \int_{S} \frac{1}{2}\left(u_{i} n_{j}+u_{j} n_{i}\right) d S
$$

may be expressed in terms of the volume average subvolume contributions through the use of Gauss theorem and the contributions due to the displacement discontinuities, yielding

$$
\begin{aligned}
\bar{\varepsilon}_{i j} & =\frac{1}{\mathrm{~V}} \int_{V} \varepsilon_{i j}(\mathbf{x}) d V-\frac{1}{2 \mathrm{~V}} \int_{S_{d}}\left(\delta u_{i} n_{j}+\delta u_{j} n_{i}\right) d S \\
& =\sum_{q=1}^{N_{q}} v_{(q)} \bar{\varepsilon}_{i j}^{(q)}-\frac{1}{2 \mathrm{~V}} \sum_{q=1}^{N_{q}^{*}} l_{d}^{(q)}\left(\delta \hat{u}_{i}^{(q)} n_{j}+\delta \hat{u}_{j}^{(q)} n_{i}\right)
\end{aligned}
$$

where $N_{q}^{*}$ is the number of damaged interfaces, $l_{d}^{(q)}$ is the length of the damaged interface and $\delta \hat{u}_{i}^{(q)}$ are the surface-averaged displacement discontinuities. This formula will be employed 
to verify the correctness of the implemented CZM and the accuracy of the CZM-based solution for the investigated unit cell problems.

\subsection{Verification}

The implemented CZM capability is first verified in the linear elastic region by comparison with an exact elasticity solution for an inclusion surrounded by a linear interface of zero thickness that obeys the same constitutive law as CZM before the onset of unloading. The inclusion is embedded in an infinite matrix and subjected to uniform far-field loading. This is the classical Eshelby problem, Eshelby (1957), with a linearly elastic flexible interface whose solution can be readily obtained by specializing the available solutions in cylindrical coordinates, (Drago and Pindera 2008). Two types of far-field loading conditions are applied: in-plane far-filed loading, $\sigma_{22}^{\infty}$ and out-of-plane shear, $\varepsilon_{12}^{\infty}$.

\subsubsection{In-Plane Far-Field Loading}

For the Eshelby in-plane problem, the displacement field in the inclusion and the matrix under plain strain conditions that satisfies the Navier's equations and far-field loading condition reads,

$$
\begin{gathered}
u_{r}^{f}(r, \theta)=A_{10}^{f} r-\left[2 v_{f} /\left(9-6 v_{f}\right) A_{12}^{f} r^{3}+A_{22}^{f} r\right] \cos 2 \theta \\
u_{\theta}^{f}(r, \theta)=\left(A_{12}^{f} r^{3} / 3+A_{22}^{f} r\right) \sin 2 \theta
\end{gathered}
$$

and

$$
\begin{gathered}
u_{r}^{m}(r, \theta)=A_{10}^{m} r+A_{20}^{m} / r \\
+\left(-A_{22}^{m} r-A_{32}^{m} r^{-3} / 3+2\left(1-v_{m}\right) /\left(1-2 v_{m}\right) A_{42}^{m} r^{-1}\right) \cos 2 \theta \\
u_{\theta}^{m}(r, \theta)=\left(A_{22}^{m}-A_{32}^{m} r^{-3} / 3-A_{42}^{m} r^{-1}\right) \sin 2 \theta
\end{gathered}
$$

The unknown coefficients $A_{10}^{m}$ and $A_{22}^{m}$ are obtained from the far-field loading condition

$$
\sigma_{r r}^{m}(r \rightarrow \infty, \theta)=1 / 2 \sigma_{22}^{\infty}(1-\cos 2 \theta)
$$

and the remaining six unknown coefficients are determined from displacement discontinuity conditions at the fiber/matrix interface $r=a$,

$$
u_{r}^{m}(a, \theta)-u_{r}^{f}(a, \theta)=u_{r}^{*} \text { and } u_{\theta}^{m}(a, \theta)-u_{\theta}^{f}(a, \theta)=u_{\theta}^{*}
$$

where the interfacial constitutive relations are

$$
\sigma_{r r}^{i n t}=k_{r}^{*} u_{r}^{*} \quad \text { and } \quad \sigma_{\theta \theta}^{i n t}=k_{\theta}^{*} u_{\theta}^{*}
$$

and interfacial traction continuity conditions 


$$
\sigma_{r r}^{f}(a, \theta)=\sigma_{r r}^{m}(a, \theta) \text { and } \sigma_{r \theta}^{f}(a, \theta)=\sigma_{r \theta}^{m}(a, \theta)
$$

For comparison purposes, we employ the uncoupled CZM separation law with $k_{r}^{*}=k_{\theta}^{*}$ and choose fiber and matrix material parameters given in Table 2.1 which do not produce cohesive zone interpenetration due to the applied horizontal loading. The unit cell constructed for FVDAM computations for comparison with the modified Eshelby solution is shown in Figure 2.5 , together with a close-up of the interfacial region. The fiber radius is one, producing volume fraction for this unit cell of 0.05 , and the discretization employed is $120 \times 120$ subvolumes. Figure 2.6 illustrates comparison of the interfacial displacement discontinuities along radial and tangential directions under loading by $\sigma_{22}^{\infty}=1 \mathrm{MPa}$, and plane strain condition $\bar{\varepsilon}_{11}=0$ along the fiber direction. As observed, the FVDAM results coincide with the modified Eshelby solution nearly everywhere around the entire fiber/matrix interface both in the radial and tangential directions. Similar observation holds for the normal and tangential traction distributions (not shown). The corresponding full-field stress distributions in Cartesian coordinates, $\sigma_{22}\left(y_{2}, y_{3}\right)$, $\sigma_{23}\left(y_{2}, y_{3}\right)$ and $\sigma_{33}\left(y_{2}, y_{3}\right)$, are given in Figure 2.7 and demonstrate the accuracy of the FVDAM predictive capability with the newly incorporated CZM in the linearly elastic range. We note that the $\sigma_{22}(0, a)$ stress concentration is substantially less than 3 because the fiber/matrix Young's modulus mismatch is just $1 / 4$ for this case and $k_{r}^{*}=10^{6} \mathrm{MPa} /$ unit length. To demonstrate that the FVDAM theory with the implemented CZM can reproduce the well-known Kirsch solution which produces the stress concentration factor of 3 at the apex of the hole under horizontal loading, we can either reduce the interfacial stiffness to a negligible level or reduce the fiber Young's modulus without changing the interfacial stiffness such that the fiber approximates a circular hole. Both approaches were taken. Figure 2.8 compares the normal and tangential stress distributions for the case when the interfacial stiffness has been degraded to a small number, namely $k_{r}^{*}=3 \mathrm{MPa} /$ unit length. As observed, the stress magnification of 3 is obtained as in the Kirsch problem. The same distributions are obtained when the inclusion properties are reduced to a very small number and the interfacial stiffness is kept at $k_{r}^{*}=$ $3 \times 10^{6} \mathrm{MPa} /$ unit length. 
Table 2.1 Elastic parameters for the modified Eshelby problem.

\begin{tabular}{|c|c|c|c|}
\hline Material & $E(\mathrm{GPa})$ & $v$ & $k_{r}^{*}=k_{\theta}^{*}(\mathrm{MPa} /$ length $)$ \\
\hline Inclusion & 100 & 0.35 & --- \\
\hline Matrix & 400 & 0.25 & --- \\
\hline Interface & ---- & ---- & $3.0 \times 10^{6}$ \\
\hline
\end{tabular}

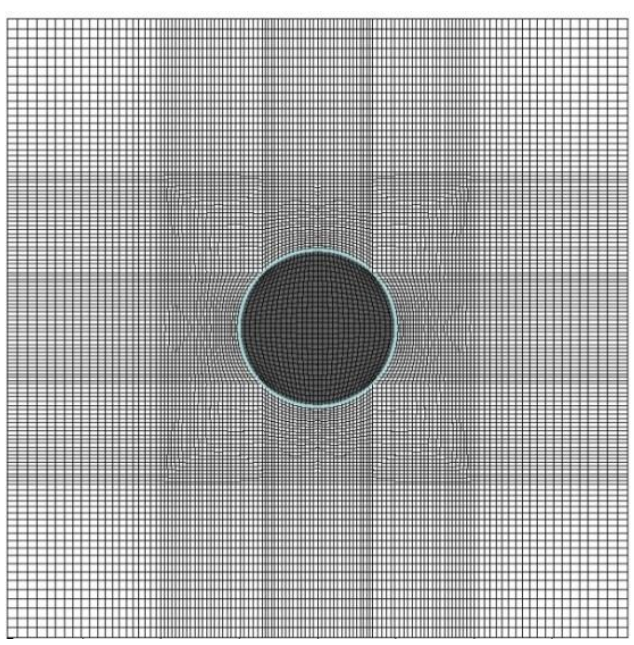

Unit cell geometry

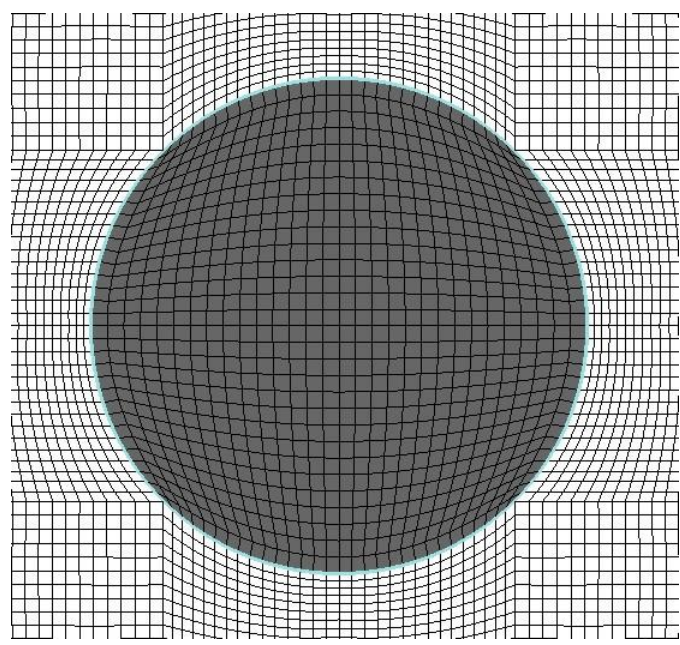

Detailed region near the inclusion

Figure 2.5 Unit cell geometry containing 0.05 fiber volume fraction (left) and a detailed close-up (right) used for comparison with the Eshelby solution for an inclusion with a linear interface in an infinite matrix.
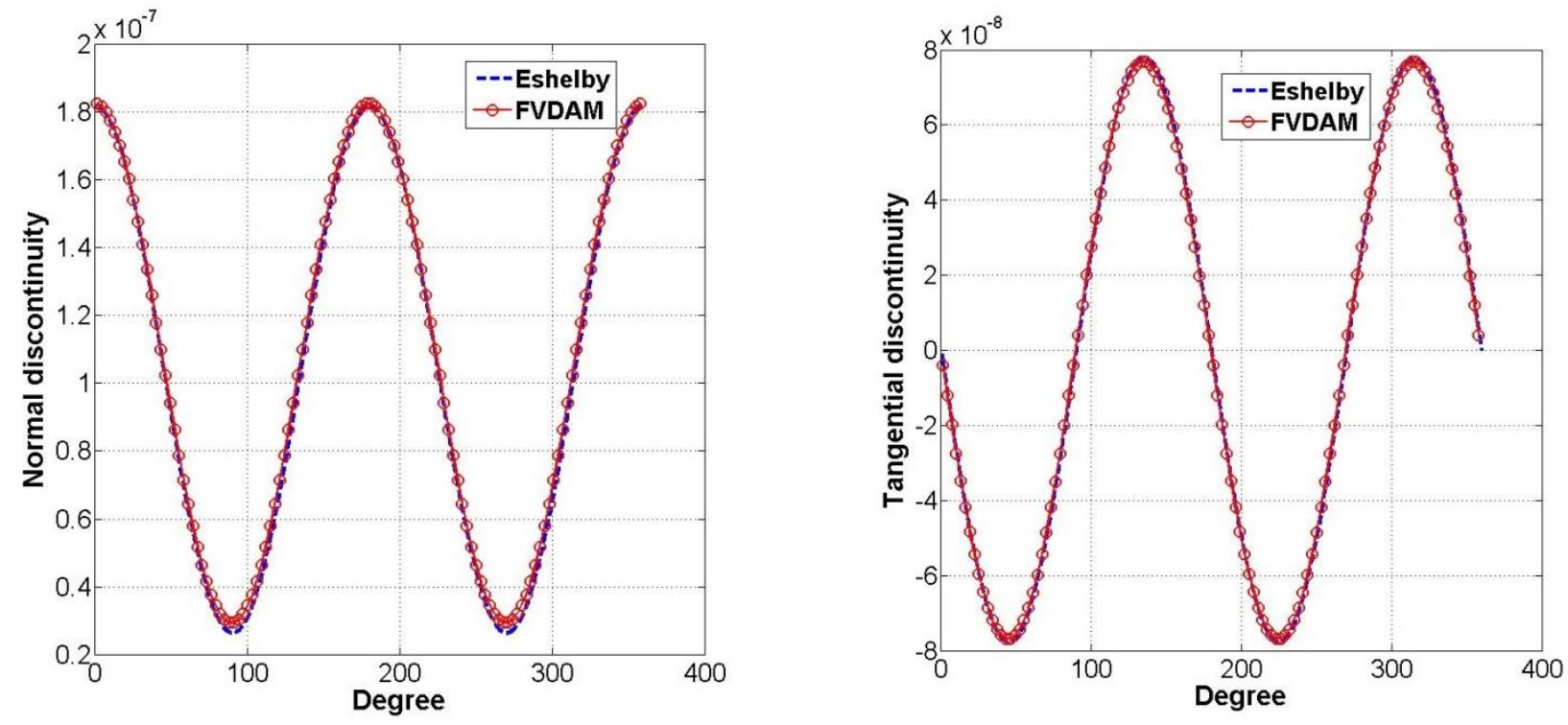

Figure 2.6 Comparison of radial and tangential displacement discontinuities around the fiber/matrix interface obtained from dilute FVDAM and Eshelby solutions. 


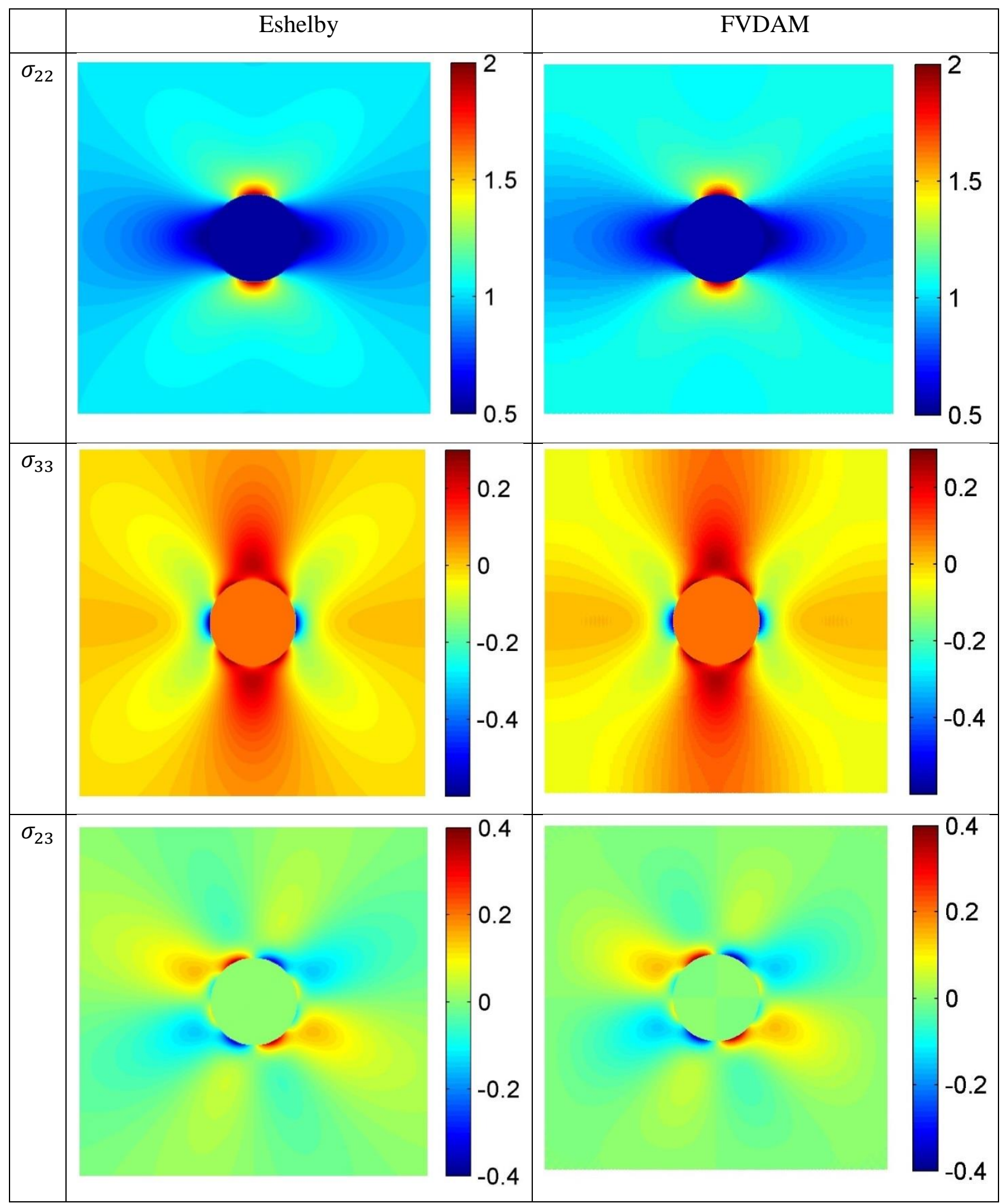

Figure 2.7 Comparison of normal and tangential stress fields in the region occupied by the unit cell obtained from dilute FVDAM and Eshelby solutions. 


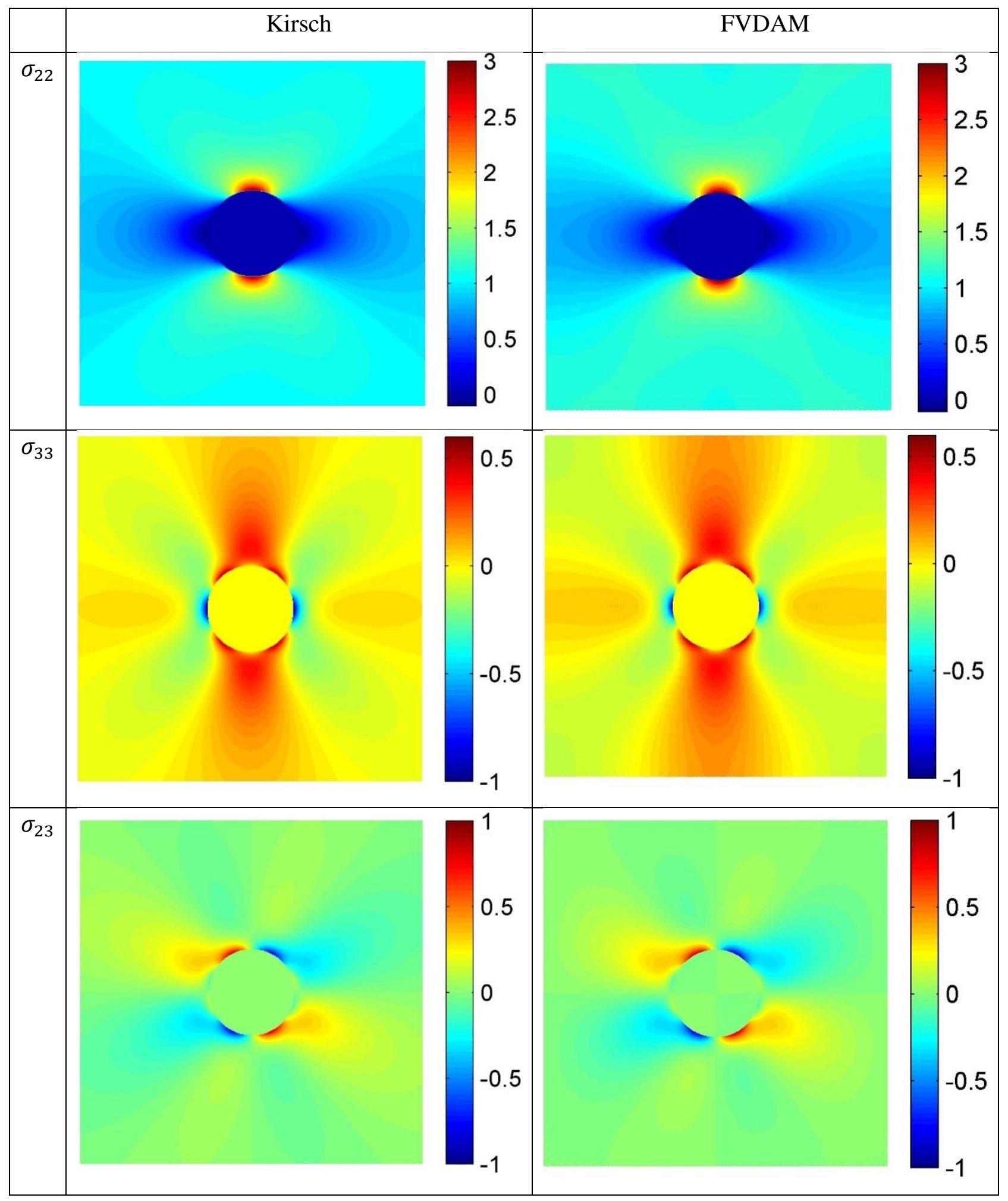

Figure 2.8 Comparison of normal and tangential stress fields in the region occupied by the unit cell obtained from dilute FVDAM and Eshelby solutions with the interfacial properties set to simulate the Kirsch solution. 


\subsubsection{Out-of-Plane Far-Field Loading}

For out-of-plane shear loading, $\varepsilon_{12}^{\infty}$, the displacement field in the inclusion and the matrix satisfies the following equations:

$$
\begin{gathered}
u_{z}^{f}(r, \theta)=B_{f} r \cos \theta-\varepsilon_{12}^{\infty} r \cos \theta \\
u_{z}^{m}(r, \theta)=\left(B_{m} r+C_{m} / r\right) \cos \theta-\varepsilon_{12}^{\infty} r \cos \theta
\end{gathered}
$$

The unknown coefficient, $B_{m}$ can be obtained from far-field condition. The other two unknown coefficients are determined from displacement discontinuity conditions at the fiber/matrix interface, $r=a$, where the interfacial constitutive relations are

$$
u_{z}^{m}(a, \theta)-u_{z}^{f}(a, \theta)=u_{z}^{*}
$$

where the interfacial constitutive relation is

$$
\sigma_{z}^{i n t}=k_{z}^{*} u_{z}^{*}
$$

and interfacial traction continuity condition is

$$
\sigma_{z}^{f}(a, \theta)=\sigma_{z}^{m}(a, \theta)
$$

Two sets of results with different interfacial properties are presented for comparison purpose. Figures 2.9-2.10 show the results when interfacial stiffness is $k_{z}^{*}=3 \times 10^{6} \mathrm{MPa} / \mathrm{unit}$ length and Figure 2.11 shows the comparison when interfacial stiffness is $k_{z}^{*}=3 \mathrm{MPa} / \mathrm{unit}$ length. The same unit cell, discretization, fiber/matrix properties are used as in the case for inplane verification and the applied loading is $\bar{\varepsilon}_{12}=0.02 \%$. Examining Figures 2.9-2.11, we see that the results have very good agreement between analytical solutions and FVDAM's results, and further when the interfacial properties are extremely weak, the stress field in the inclusion is almost zero due to the loss of load transfer capability from the matrix to the inclusion. 

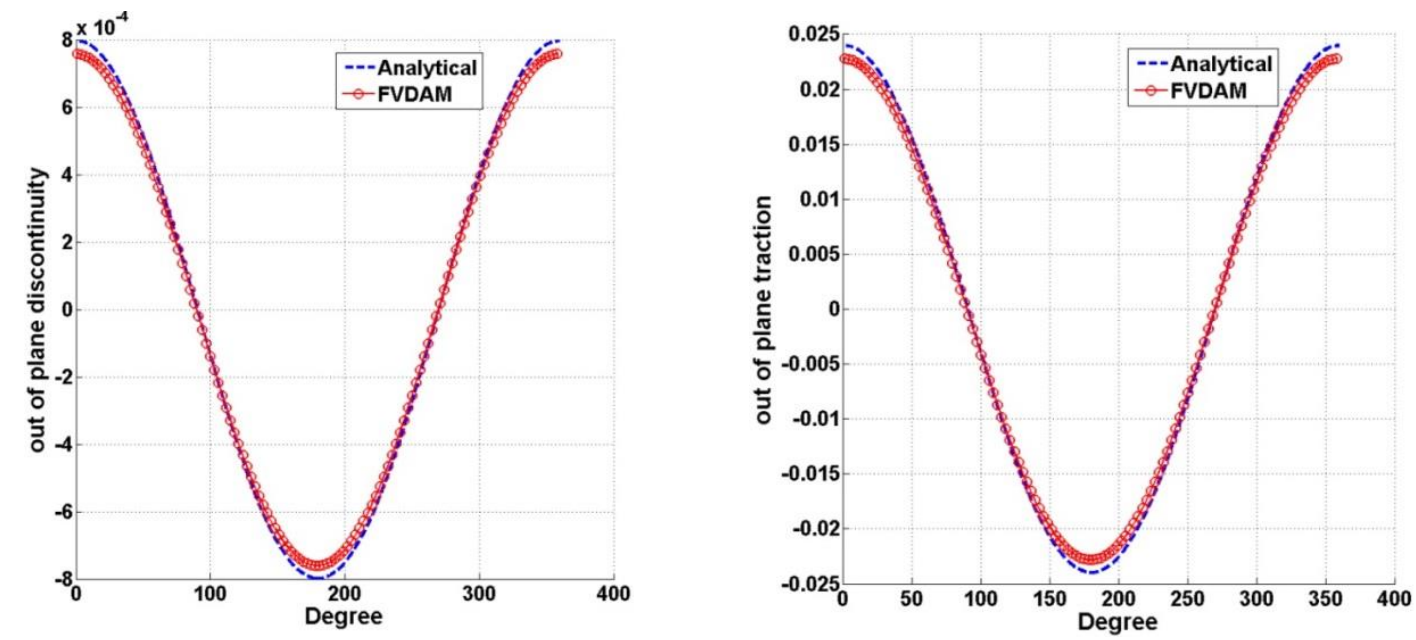

Figure 2.9 Comparison of displacement discontinuity and traction around the fiber/matrix interface obtained from dilute FVDAM and Eshelby solutions.

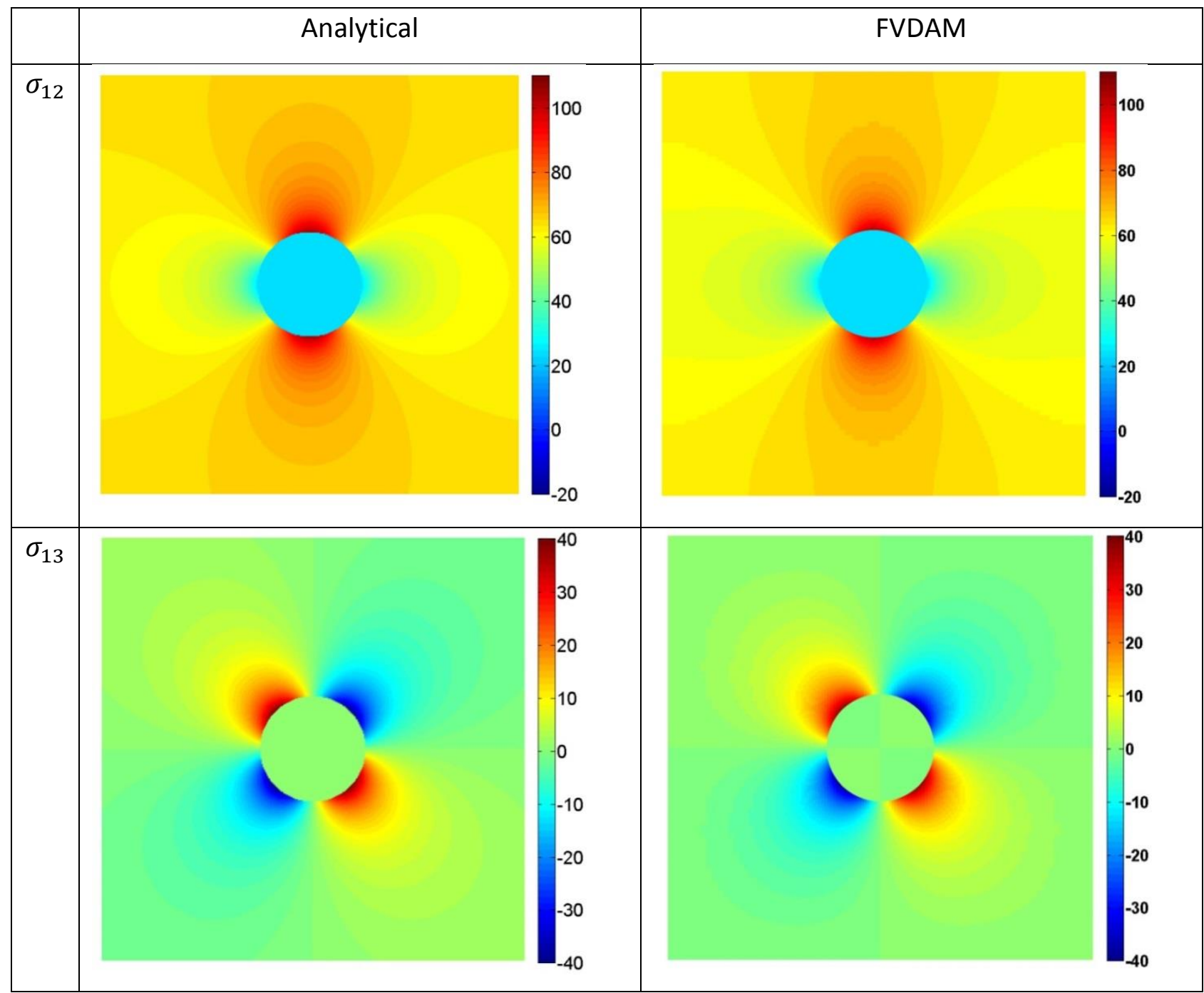

Figure 2.10 Comparison of out-of-plane shear stress fields in the region occupied by the unit cell obtained from dilute FVDAM and Eshelby solutions. 


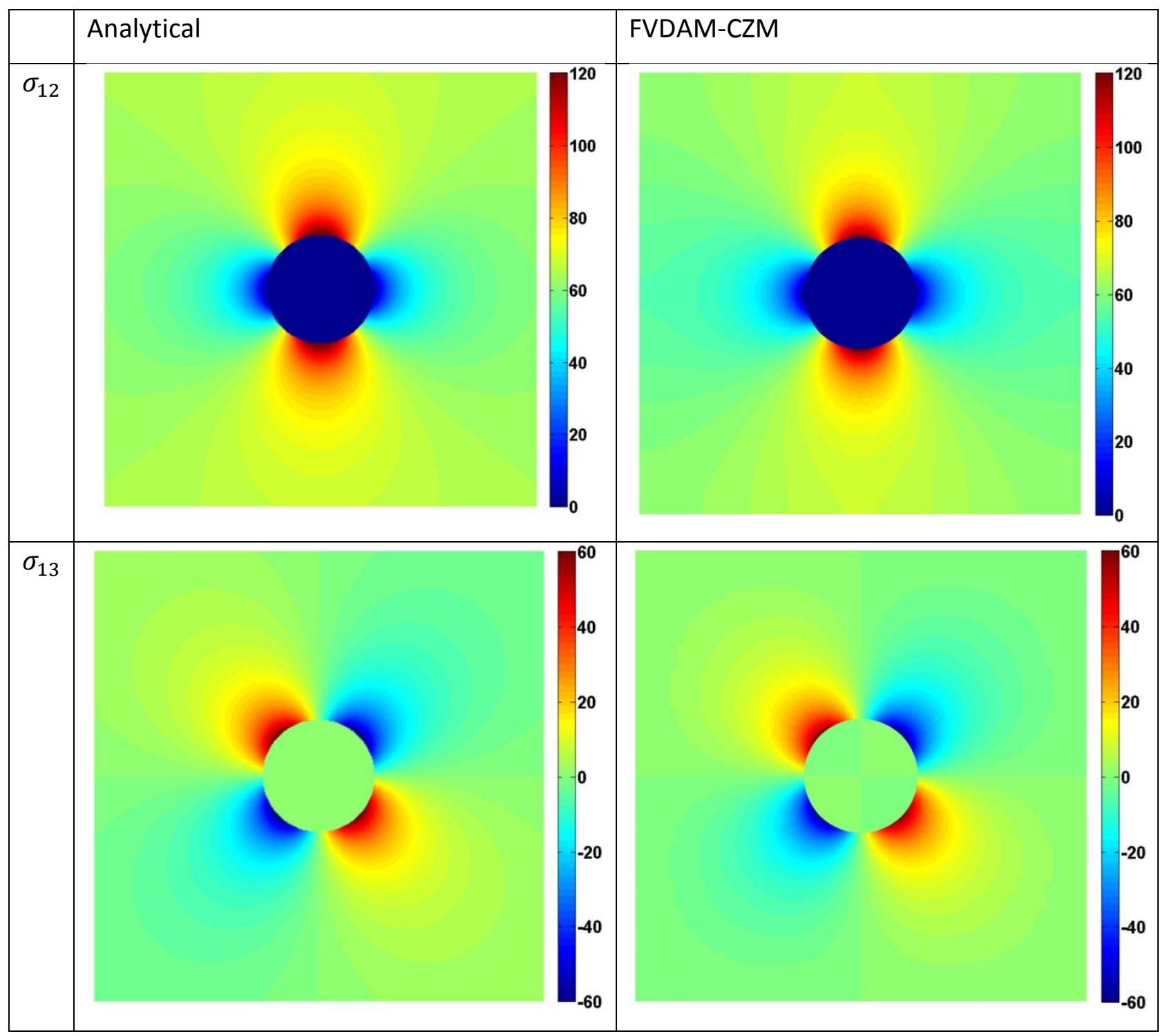

Figure 2.11 Comparison of out-of-plane shear stress fields in the region occupied by the unit cell obtained from dilute FVDAM and Eshelby solutions with extremely small interfacial properties.

\subsection{Summary}

The Cohesive Zone Model (CZM) has been incorporated into the parametric FVDAM theory in order to accommodate damage evolution based on displacement discontinuity functions under inplane loading and out-of-plane shear loading. With the aid of displacement discontinuity, the system of governing system of equations that control the response of a periodic composite undergoing damage response is separated into primary and auxiliary system of equations, with the primary system solved just once. To analyze a damage evolution problem, only the auxiliary 
system of equations needs to be solved iteratively without re-assembling the global system of equations. The added benefit is that the propagation of stationary cracks, and calculation of concomitant energy release rates, is also treated within the same framework upon modifying the auxiliary system of equations in a straightforward manner. Another important feature of the framework is the ease with which compressive stress normal to the interface undergoing damage is treated. In contrast with typical finite-element approaches which rely on artificially large interfacial stiffness when the normal stress becomes compressive, in the developed approach the governing equations for a damaged interface in the auxiliary system of equations are simply eliminated. Further, the implemented CZM has been verified in the linear elastic region upon comparison with modified Eshelby solutions for both in-plane loading and out-of-plane shear loadings, which accounts for an elastic cohesive interface between the fiber and matrix phases. Both the interfacial separations and tractions around the interface and the full-field stress distributions have been captured by the extended FVDAM with fidelity comparable to the elasticity solution. 


\section{Chapter 3}

\section{Assessment of CZM-FVDAM and Abaqus}

\section{Predictive Capabilities}

\subsection{Introduction}

In Chapter 2, CZM-FVDAM's ability and accuracy in predicting interfacial separation and traction were verified upon comparison with the modified Eshelby solution when the cohesive law remained in the linearly elastic stage. In this chapter, CZM-FVDAM's ability and accuracy in predicting interfacial debonding of fiber-reinforced materials is critically and fully assessed against the commercial finite-element code, Abaqus extensively used by the design and development and research communities. The chosen material system is SiC/Ti in which premature fiber/matrix interfacial debonding occurs at low transverse normal stresses due to fiber/matrix interface degradation produced by a fabrication-induced chemical reaction. Hence, this composite system is a realistic candidate to assess the debonding modeling capability of the finite-volume and finite-element based approaches.

In Section 3.2, unit cell geometry and discretization, cohesive law used in Abaqus and FVDAM and the manner of applying periodicity conditions are described. In Section 3.3, the effect of interfacial stiffness under compressive normal stress employed in Abaqus is investigated. The choice of interfacial stiffness affects the correctness of simulation results because of the extent of fictitious material interpenetration that may occur if the stiffness is too low. Conversely, unnecessarily large interfacial stiffness may produce incorrect results. In Section 3.4, interfacial separation predicted by Abaqus and FVDAM simulations at selected segments along the fiber/matrix interface is compared under pure traction or combined normal and shear tractions. In Sections 3.5 and 3.6, detailed comparison of the homogenized response, interfacial separations, and full-field stress distributions predicted by Abaqus and FVDAM is conducted under biaxial strain loading and transverse shear loading. In Section 3.7, the effect of fiber and matrix modulus contrast is discussed. Section 3.8 is the summary and discussion. 


\subsection{Model Set-Up in CZM-FVDAM and Abaqus}

\subsubsection{Unit Cell Geometry and Discretization}

In this study, the unit cell is loaded subject to plane strain constraint and hence 2D plane strain elements CPE4 available in Abaqus are used to discretize the analysis domain. Figure 3.1 shows representative unit cell used in FVDAM and Abaqus simulations of the unidirectional SiC/Ti composite in the cross section normal to the fiber direction with the highlighted cohesive zone around the entire fiber. The elastic moduli of $\mathrm{SiC}$ fiber and titanium matrix are given in Table 3.1. The $\mathrm{SiC}$ fiber volume fraction is 0.325 , the diameter of the fiber is $142 \mu \mathrm{m}$ and the unit cell dimensions are $221 \times 221 \mu \mathrm{m}$. The unit cell used in FVDAM simulations shown on the left is discretized into $52 \times 52$ subvolumes or 2704 subvolumes in total, and the unit cell used in Abaqus simulations shown on the right is discretized into 2944 elements using similar discretization as that in FVDAM. The fiber/matrix interface in both models has the same discretization which contains 120 subvolume faces in FVDAM and 120 2D COH2D4 cohesive elements in Abaqus.

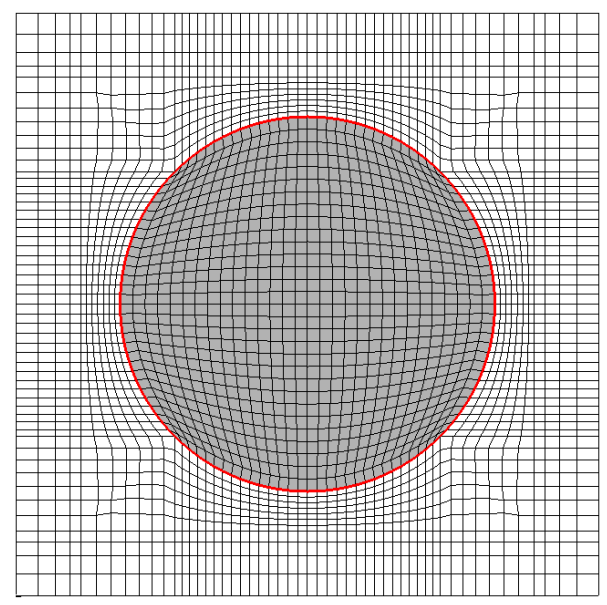

Mesh in FVDAM

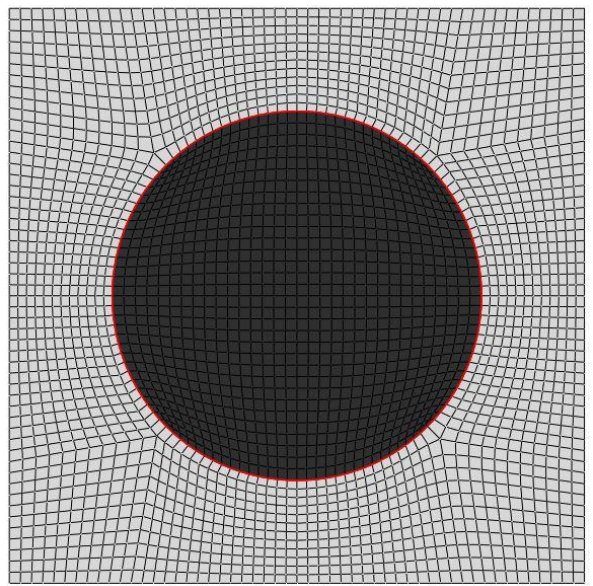

Mesh in ABAQUS

Figure 3.1 Unit cell geometry and discretization containing 0.325 fiber volume fraction used in FVDAM (left) and Abaqus (right).

The cohesive zone model in Abaqus is implemented via cohesive (interface) elements shown in Figure 3.2, which are compatible with regular solid elements. The relative motion of the bottom and top face of the cohesive element is characterized by interfacial opening or closing of the interface along thickness direction and transverse shearing. Figure 3.3 shows the node ordering and face numbering of the cohesive element COH2D4 and positions of integration points and element centroid. The bulk or solid elements are connected to cohesive elements by shared nodes. When traction-separation relation is specified for the cohesive element, which will 
be discussed in sub-section 3.2.2, there are two stress and two strain outputs. These are direct through-thickness and transverse shear stresses, S22 and S23, and the corersponding strains, E22 and E23, respectively. The output can be obtained from element nodes, integration points, and the element centroid. In the employed Abaqus analysis, the cohesive element thickness is zero, the strains E22 and E12 are equal to the normal and tangential separations $\delta_{n}$ and $\delta_{t}$, respectively, and the stress S22 and S12 are equal to the normal and tangential tractions $t_{n}$ and $t_{t}$.

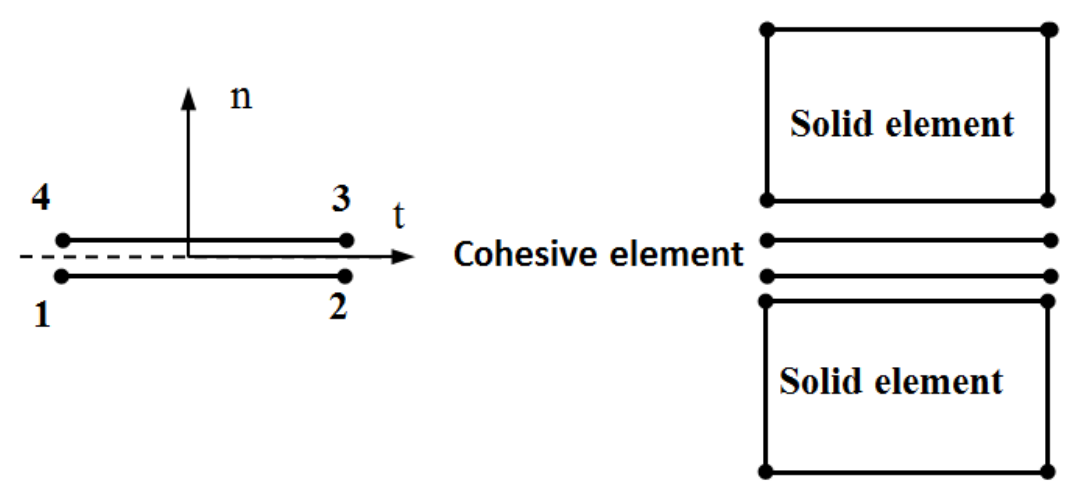

Figure 3.2 Schematics of 2D cohesive elements.

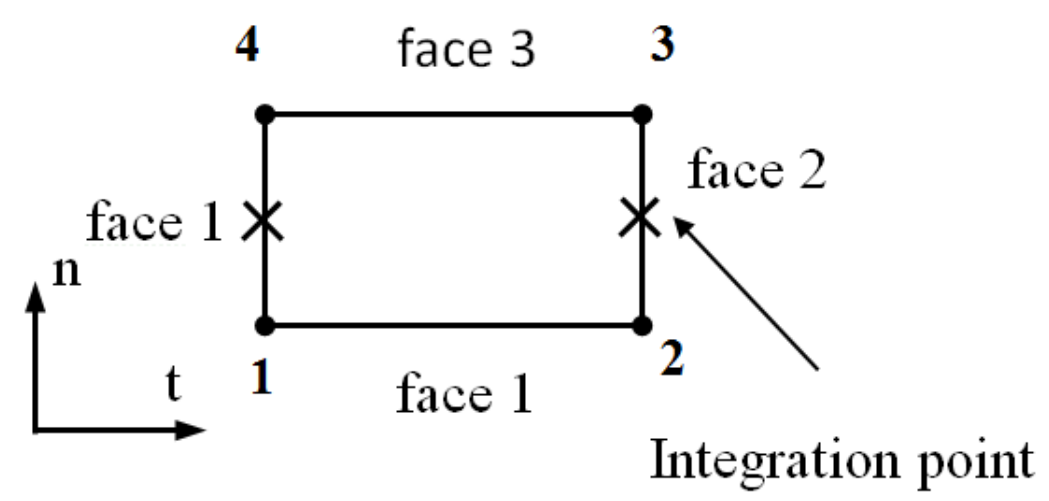

Figure 3.3 Node ordering and face numbering of cohesive element COH2D4 and positions of integration points and element centroid.

Table 3.1 Elastic properties of the SiC fibers and Ti matrix.

\begin{tabular}{|c|c|c|}
\hline & $\mathrm{E}(\mathrm{GPa})$ & $v$ \\
\hline Fiber & 400 & 0.25 \\
\hline Matrix & 92.38 & 0.35 \\
\hline
\end{tabular}




\subsubsection{Cohesive Law in Abaqus}

Cohesive law characterizes the interfacial traction-separation relation, which can be generally divided into three stages: elastic opening, interfacial degradation, final separation. Coupled bilinear cohesive law implemented into the thesis is widely used by many researchers, Eqs. (2.27-2.28). To the best of author's knowledge, in Abaqus the cohesive law with uncoupled elastic traction-separation behavior before damage initiation, with maximum nominal stress criterion for damage initiation, Eq. (3.1) and with linear damage evolution, Eq. (3.2) is the closest one to the coupled bilinear cohesive law.

$$
\max \left\{\frac{\left\langle t_{n}\right\rangle}{t_{n}^{o}}, \frac{t_{t}}{t_{t}^{o}}\right\}=1
$$

where $<>$ is the Macaulay bracket, $t_{n}^{o}$ is the interfacial strength in normal direction and $t_{t}^{o}$ is the interfacial strength in transverse shear direction.

$$
\begin{gathered}
t_{n}=\left\{\begin{array}{l}
(1-D) \bar{t}_{n}, \quad \bar{t}_{n} \geq 0 \\
\bar{t}_{n}, \quad \text { other wise }
\end{array}\right. \\
t_{t}=(1-D) \bar{t}_{t}
\end{gathered}
$$

where $\bar{t}_{n}, \bar{t}_{t}$ are the stress components predicted by the elastic traction-separation behavior for the current strains without damage and $\mathrm{D}$ is a scalar damage variable, which represents the overall damage in the material and has the following expression, Eq. (3.3), with linear softening assumption, Figure 3.4 .

$$
D=\frac{\delta_{m}^{f}\left(\delta_{m}^{\max }-\delta_{m}^{0}\right)}{\delta_{m}^{\max }\left(\delta_{m}^{f}-\delta_{m}^{0}\right)}
$$

where $\delta_{m}^{\max }$ refers to the maximum value of the effective displacement attained during the loading history, $\delta_{m}^{0}$ is the effective displacement at damage initiation and $\delta_{m}^{f}$ is the effective displacement at complete failure. The effective displacement and traction are defined in Eq. (3.4).

$$
\begin{aligned}
& \delta_{m}=\sqrt{\left\langle\delta_{n}\right\rangle^{2}+\delta_{t}^{2}} \\
& t_{m}=\sqrt{\left\langle t_{n}\right\rangle^{2}+t_{t}^{2}}
\end{aligned}
$$




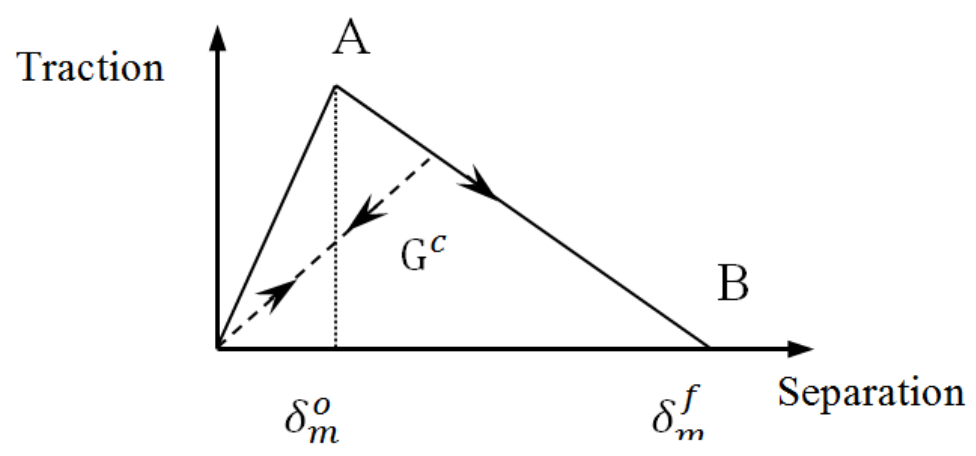

Figure 3.4 Linear damage evolution.

Since in the Abaqus Manual 6.14, the cohesive law is described with a mixture of text and formula, it is more difficult to accurately interpret the law than that described by a pure math formula. So the best way to compare the cohesive laws is to plot the interfacial tractionseparation relation for an individual interface segment obtained from FVDAM and analyses. Such comparison will be presented and discussed in detail in Section 4. 4.

In addition, Section 3.2.1 illustrates that the output of tractions and displacement separations can be specified to different positions in the element, such as element nodes, integration points, and element centroid. In Abaqus, the user has a choice to specify the position at which the corresponding traction and displacement separation quantities will be used for the cohesive law, with the default position setting the centroid, which is used in the current analysis. Since in FVDAM the interfacial quantities are surface-average quantities, the corresponding position is at the middle of subvolume face. With the centroid setting in Abaqus, outputs of interfacial quantities from two approaches can be compared directly without additional postprocessing because the cohesive element thickness is zero.

\subsubsection{Imposition of Periodic Boundary Conditions in Abaqus}

In FVDAM, periodic boundary conditions are incorporated into the theory intrinsically, see Figure 2.3, whereas in Abaqus the condition is imposed by constraining the nodal displacements along the unit cell boundary pairs via coupling equations, (Kulkarni 2012, Yang 2016). In this chapter the unit cell subjected to two types of loading is analyzed: one is bi-axial strain loading with non-zero $\bar{\varepsilon}_{33}$ and $\bar{\varepsilon}_{22}$, which has a fixed ratio of $-1 / 2$ and another one is transverse shear loading with only non-zero $\bar{\varepsilon}_{23}$ and those two types of loadings are applied in all analysis. Figure 3.5 is a schematic figure of the square unit cell with fiber in the center. For the bi-axial strain 
loading the boundary conditions described in Eq. (3.5) are applied, and for transverse shear loading the boundary conditions described in Eq. (3.6) are applied.

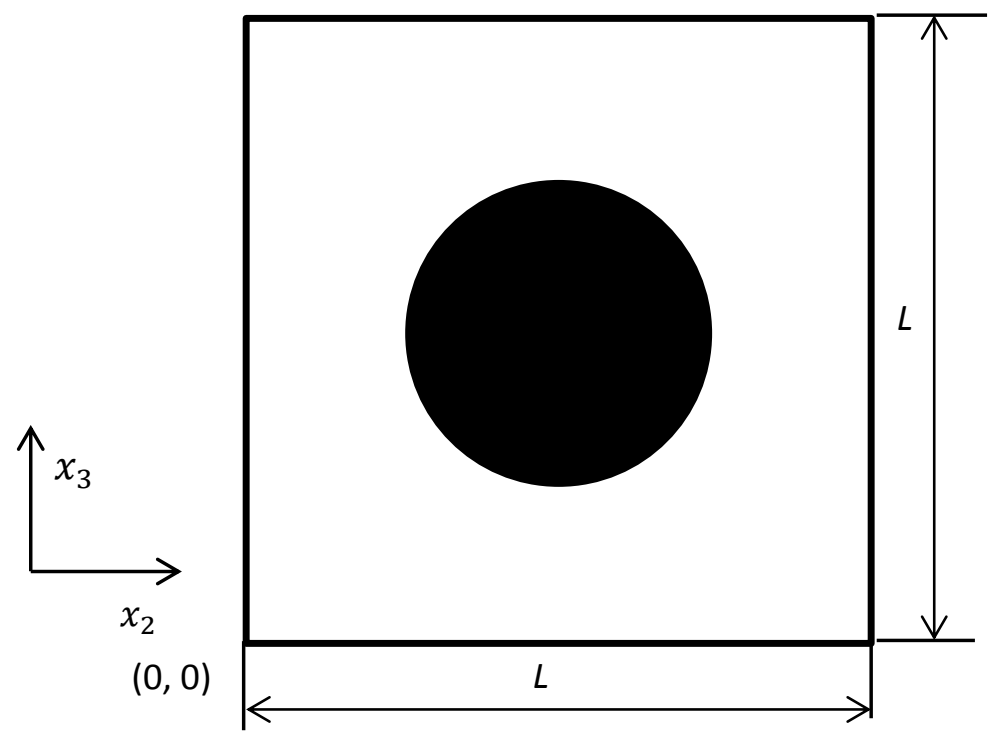

Figure 3.5 Schematic figure of square unit cell with fiber in center under plane strain condition.

$$
\begin{gathered}
u_{i}(0,0)=0, i=2,3 \\
u_{2}\left(L, x_{3}\right)-u_{2}\left(0, x_{3}\right)=\bar{\varepsilon}_{22} L ; u_{3}\left(L, x_{3}\right)-u_{3}\left(0, x_{3}\right)=0 \\
u_{2}\left(x_{2}, L\right)-u_{2}\left(x_{2}, 0\right)=0 ; u_{3}\left(x_{2}, L\right)-u_{3}\left(x_{2}, 0\right)=\bar{\varepsilon}_{33} L \\
u_{i}(0,0)=0, i=2,3 \\
u_{2}\left(L, x_{3}\right)-u_{2}\left(0, x_{3}\right)=0 ; u_{3}\left(L, x_{3}\right)-u_{3}\left(0, x_{3}\right)=\bar{\varepsilon}_{23} L \\
u_{2}\left(x_{2}, L\right)-u_{2}\left(x_{2}, 0\right)=\bar{\varepsilon}_{23} L ; u_{3}\left(x_{2}, L\right)-u_{3}\left(x_{2}, 0\right)=0
\end{gathered}
$$

\subsection{Effect of Interfacial Stiffness under Compressive Normal Stress}

In the actual implementation of the cohesive zone model, one important question that needs to be addressed is how to treat the interfacial behavior when the interface is under normal compressive stress. The most common approach is to use large stiffness along normal direction to resist interpenetration, which is also used by the commercial finite element packages such as Abaqus and Ansys. In contrast, when the interface is under compressive normal stress, the corresponding auxiliary equation in FVDAM, Eq. (2.25), for that interface along the normal direction is eliminated from the system. In this way, traction-separation relation in the normal direction is 
replaced by the corresponding traction and displacement continuity conditions without the need to re-assemble the primary system of equations, with the added advantage that absence of interpenetration is guaranteed along the normal direction.

There is no general rule to determine compressive interfacial stiffness and ideally, the stiffness should be infinite producing no interpenetration. However, large interfacial stiffness potentially could create numerical problems, (Turon et al. 2007, Song 2008). Here a parametric study is conducted aimed at identifying the appropriate compressive stiffness in the conducted simulations. Interfacial parameters in Table 3.2 are employed in the analysis, which are the same as those in the modeling of fiber/matrix debonding in SiC/Ti composites in Chapter 4. In Abaqus, the default compressive interfacial stiffness is the same as tensile interfacial stiffness, $k_{n}^{0}$ and the compression factor, $f$, can be used to adjust the normal compressive interfacial stiffness, which is equal to $f * k_{n}^{0}$, as illustrated in Figure 3.6.

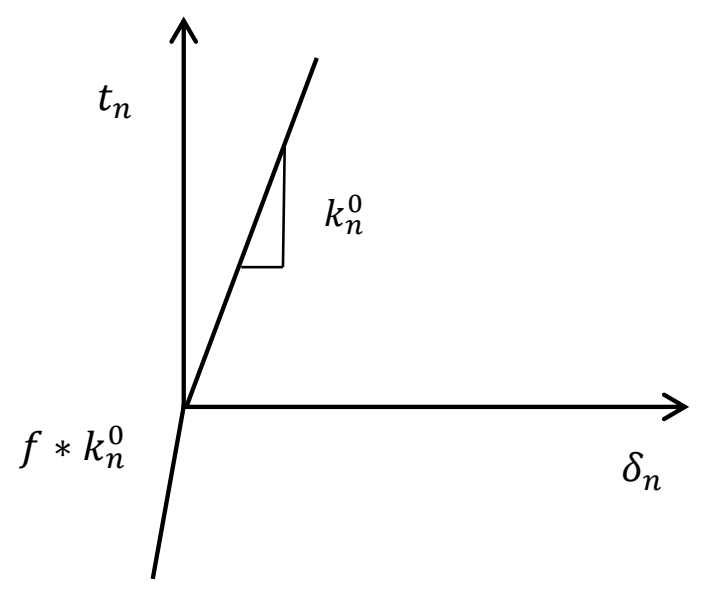

Figure 3.6 Schematic figure of interfacial stiffness and compression factor, $f$.

Table 3.2 Interfacial strength parameters for the SiC/Ti composite. Note: $k_{n}^{0}=\sigma_{\max } / \Delta_{n}^{o}$.

\begin{tabular}{|c|c|c|c|}
\hline$\sigma_{\max }=\tau_{\max }(\mathrm{MPa})$ & $k_{n}^{0}=k_{t}^{0}(\mathrm{MPa} / \mu \mathrm{m})$ & $\Delta_{n}^{0}=\Delta_{t}^{0}(\mu m)$ & $\Delta_{n}^{c}=\Delta_{t}^{c}(\mu m)$ \\
\hline 50 & 3000 & 0.0167 & 0.46 \\
\hline
\end{tabular}




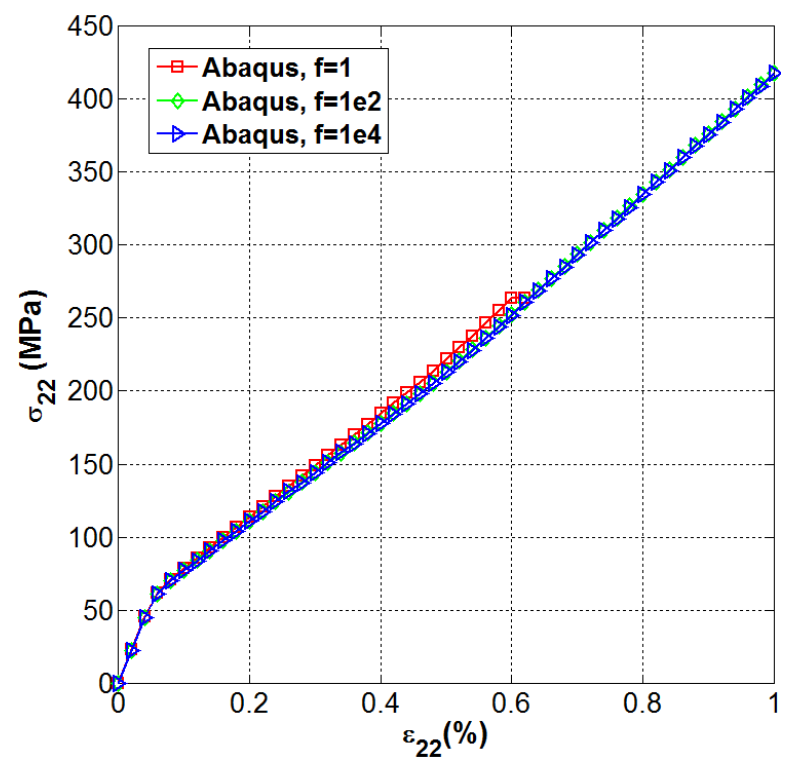

(a)

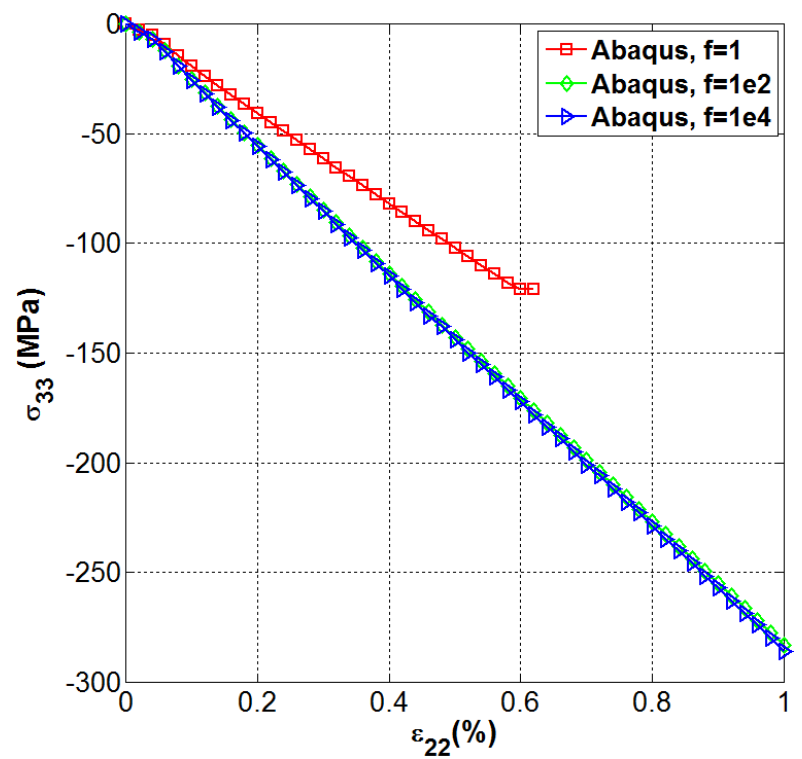

(b)

Figure 3.7 Selected homogenized responses with different compression factors in $\mathrm{x}_{2}, \mathrm{x}_{3}$ directions under biaxial strain loading.

Figure 3.7 shows the selected homogenized responses of a square unit cell under bi-axial strain loading with different compressive stiffness. The transverse shear stress $\bar{\sigma}_{23}$ is zero and the normal stress $\bar{\sigma}_{11}$ is not shown here. Boundary conditions and loading are prescribed using Eq. (3.5). The ratio between the applied strains $\bar{\varepsilon}_{33}$ and $\bar{\varepsilon}_{22}$ is $-1 / 2$. When the compression factor $f=1$, Abaqus terminates at loading step 31 due to numerical problems. When the compression factor increases to 1e2, Abaqus can complete the analysis and the homogenized responses along $\mathrm{x}_{1}, \mathrm{x}_{2}, \mathrm{x}_{3}$ directions are similar to the responses obtained with the compression factor of $1 \mathrm{e} 4$. Further, if the compression factor is increased to 1e14, Abaqus will run into numerical problems at the first loading step. Further when the compression factor is 1, then tensile homogenized responses $\bar{\sigma}_{22}$ are similar to the reponse with higher compression factors, whereas the compressive homogenized response $\bar{\sigma}_{33}$ is more compliant than the response with higher compression factors. The difference between homogenized responses with different compression factors can be explained by looking at the interfacial traction and displacement discontinuity distributions shown in Figure 3.8. As observed, normal interpenetration occurs around the top and bottom area of the fiber/matrix interface for the low compression factor and the normal traction in these areas is also lower than the normal tractions in cases with higher compression factors. The tangential tractions and displacement discontinuities are not affected by the compression factors. 
Figure 3.9 illustrates full-field stress distributions with different compression factors at step 20. The stress distributions with compression factors of 1e 2 and 1e4 are almost identical and the distributions with a compression factor of 1 are different from these two, with the high-stress distributions patterns around fiber top and bottom areas not well captured due to interpenetration.
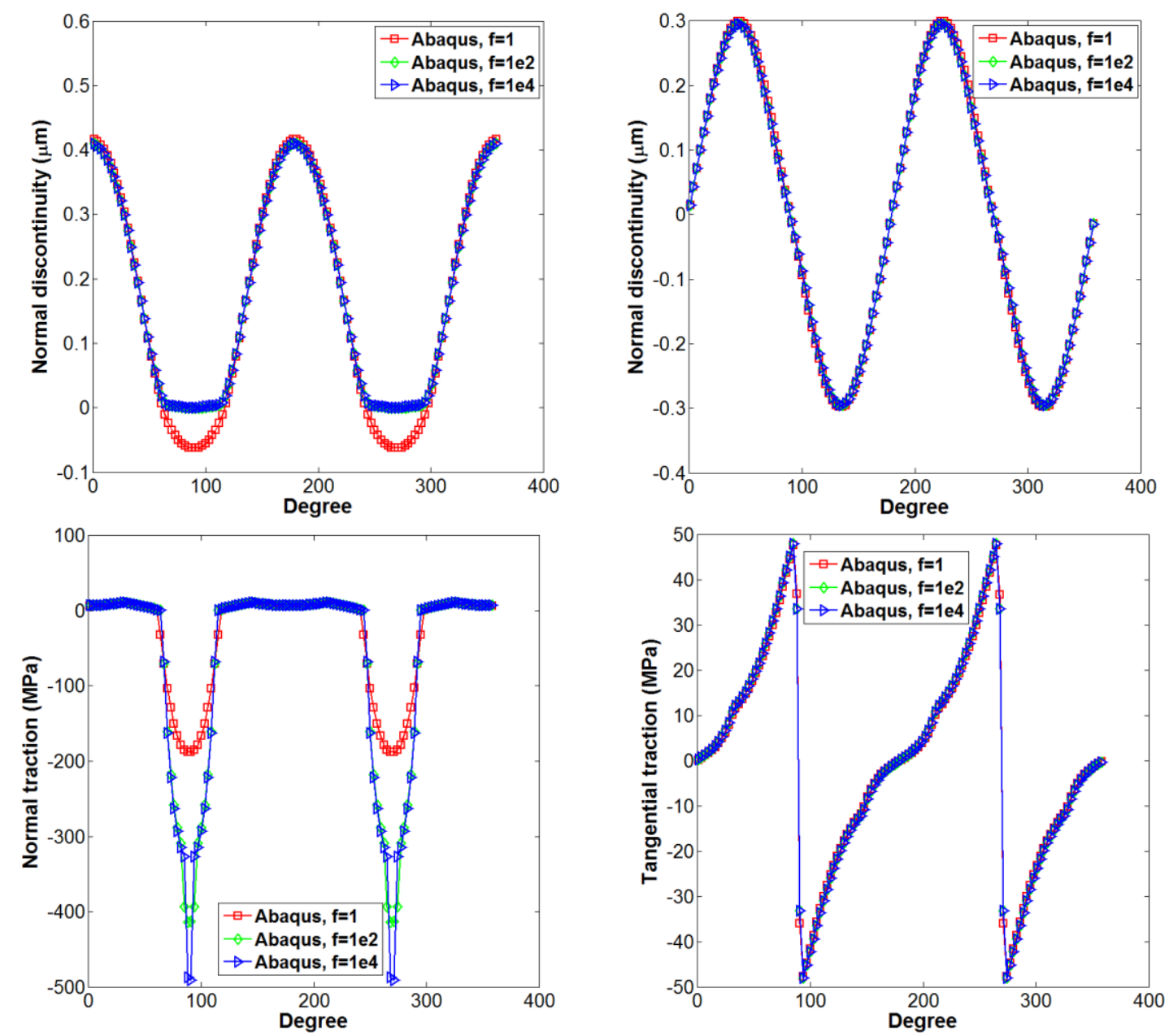

Figure 3.8 Interfacial displacement discontinuity (Left) and traction distributions (Right) around fiber/matrix interface at loading step 20. 


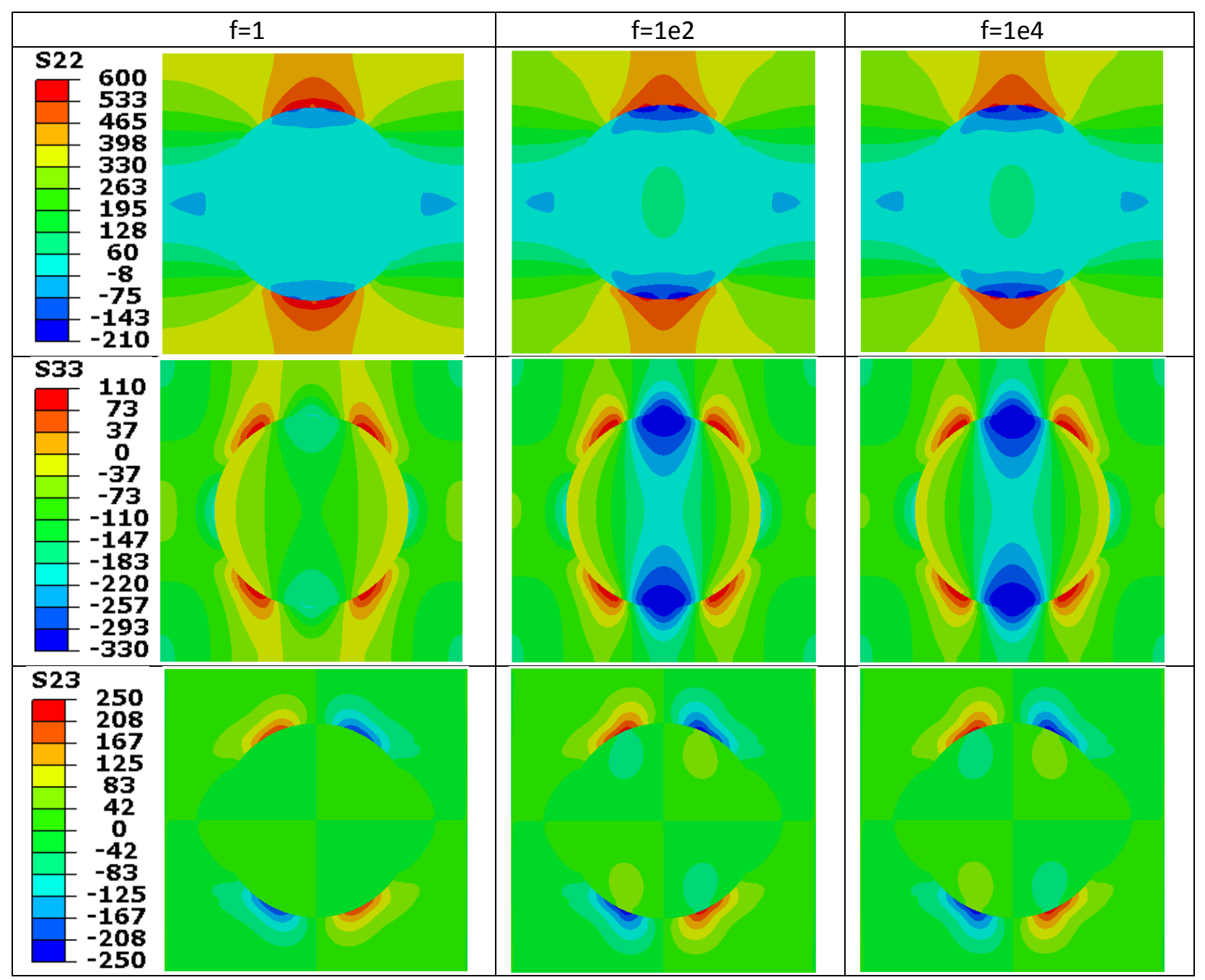

Figure 3.9 Full-field stress distributions with different compression factors at loading step20.

\subsection{Damage Evolution along Specific Interfaces}

As mentioned in Section 3.2.2, the explicit mathematical formula in the Abaqus 6.14 manual for the cohesive relation is not amenable for direct comparison with the CZM equations implemented into the FVDAM framework. Hence in this section the interfacial tractionseparation relations implemented in FVDAM and Abaqus will be illustrated in great detail by tracking the interfacial separation process for individual interfaces during simulations. A single discretized interface could fail under three types of stress states: 1) under pure normal tensile traction, 2) under pure shear traction, 3) under combined normal and shear tractions. To demonstrate these three types of interfacial failure behavior, 1st and 11th interfaces in Figure 3.10 were selected. More specifically, the 1 st interface is expected to fail under pure tensile 
loading and the 11th interface is expected to fail under combined normal and shear stress when the unit cell is under bi-axial strain loading characterized by non-zero $\bar{\varepsilon}_{22}$ and $\bar{\varepsilon}_{33}$ applied in the ratio of $-1 / 2$. Also, the 1 st interface is expected to fail under pure transverse shear stress and the 11th interface is expected to fail under combined normal and shear stresses when the unit cell is subjected to transverse shear loading characterized by non-zero shear strain $\bar{\varepsilon}_{23}$ only.

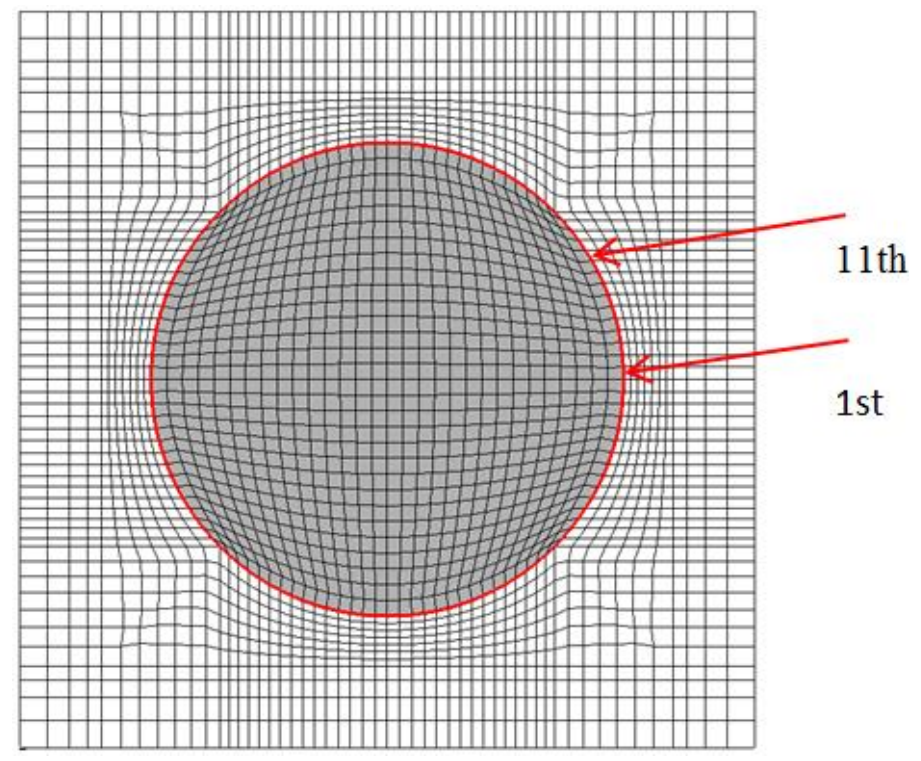

Figure 3.10 Selected interfaces for the purpose of tracking interfacial separation process.

\subsubsection{Bi-axial Strain Loading}

First, interfacial behavior of the 1st and 11th interfaces is investigated when the unit cell is subjected to bi-axial strain loading. As shown in Figure 3.11, FVDAM simulation predicts that the 1st interface separates completely at step 23 and 11th interface fails at step 24 whereas Abaqus predicts that the 1st interface separates completely at step 24 and 11th interface fails at step 26. Figures 3.12(a, b, c) compare the FVDAM and Abaqus predictions of the tractionseparation relations for the 1st interface in terms of effective traction, normal traction, and tangential traction. Since the interfacial degradation process in both cohesive laws is based on the effective displacement discontinuity, the traction quantities are plotted against the effective displacement discontinuity. The interface failure processes predicted by FVDAM and Abaqus are very close, except for the slight difference in the shear response. The failure process is dominated by normal tensile loading and the effective and normal traction responses are also the same since the contribution from shear traction is negligible. Also, we observed that the interface 
starts degrading when the interfacial strength, 50MPa, is attained and interface separates completely when final separation distance, $0.46 \mu \mathrm{m}$, is reached. These observations also verify the correct implementation of the cohesive law in FVDAM and Abaqus.

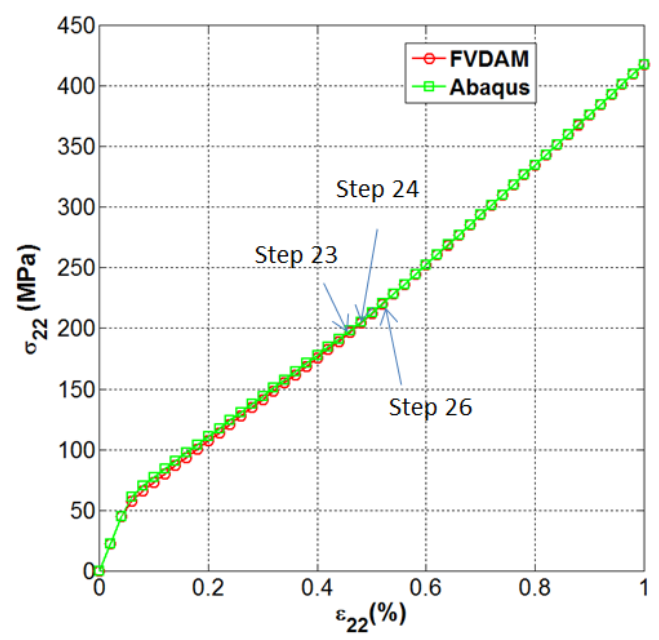

Figure 3.11 Homogenized response $\sigma_{22}$ and indication of loading steps at which the 1st and 11th interfaces separate completely.

Figures 3.12 (d, e, f) compares the FVDAM and Abaqus predictions of the tractionseparation relations for the 11th interface, which is under combined normal and shear loading, in terms of effective traction, normal traction, and tangential traction. The interfacial separation processes predicted by FVDAM and Abaqus simulations are similar in the elastic opening stage and final separation stage, with the interface failing completely when the effective displacement reaches $0.46 \mu \mathrm{m}$. The main difference lies in the interfacial softening process. According to FVDAM simulation, the interface starts softening when the effective traction attains the interfacial strength $50 \mathrm{MPa}$ and the relation between the effective traction and effective displacement relation in the softening stage is linear, as indicated in the cohesive equations, Eqs. (2.27-2.28). In contrast, Abaqus damage initiation in Abaqus is determined by maximal normal or tangential traction, indicated in Eq. (3.1), instead of the effective traction. Hence the 11th interface starts softening when the normal traction attains the interfacial strength $50 \mathrm{MPa}$ as shown in Figure 3.9(b). Because of the different manner of treating damage initiation, Abaqus predicts softening at a higher effective stress level, 65MPa in this case, compared with 50MPa predicted by FVDAM. Nonetheless, both methods predict complete interfacial failure when the effective displacement reaches $0.46 \mu \mathrm{m}$. Hence under combined tractions, the interface in Abaqus simulations will dissipate more energy than its counterpart in FVDAM, potentially 
leading to stiffer homogenized response. In the following two sections of simulation result comparison, this point will be illustrated and confirmed.

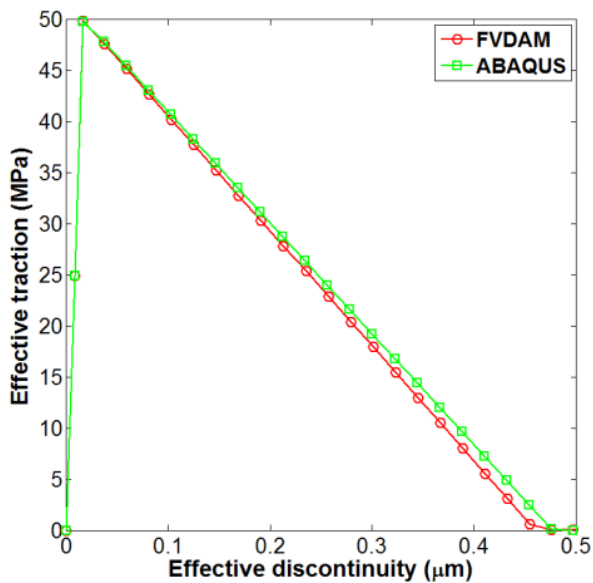

(a)

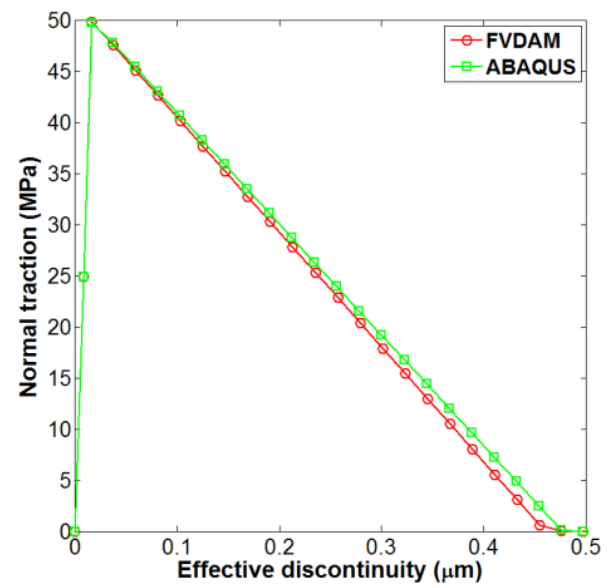

(b)

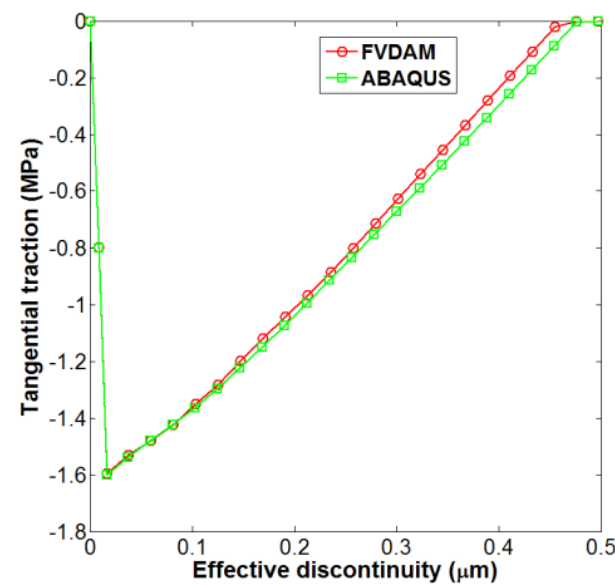

(c)

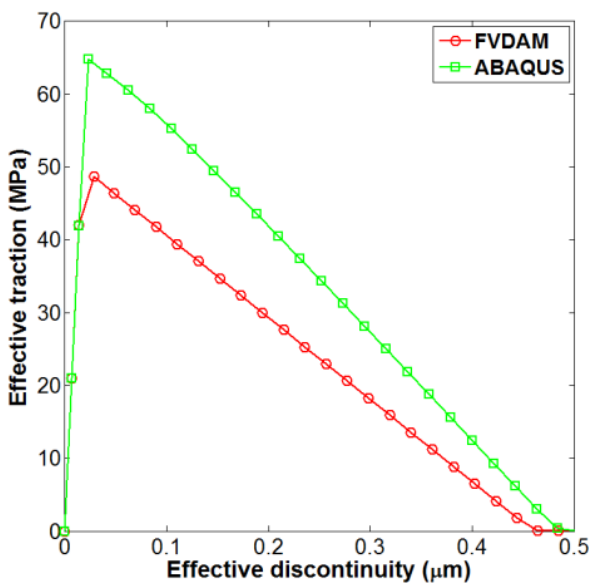

(d)

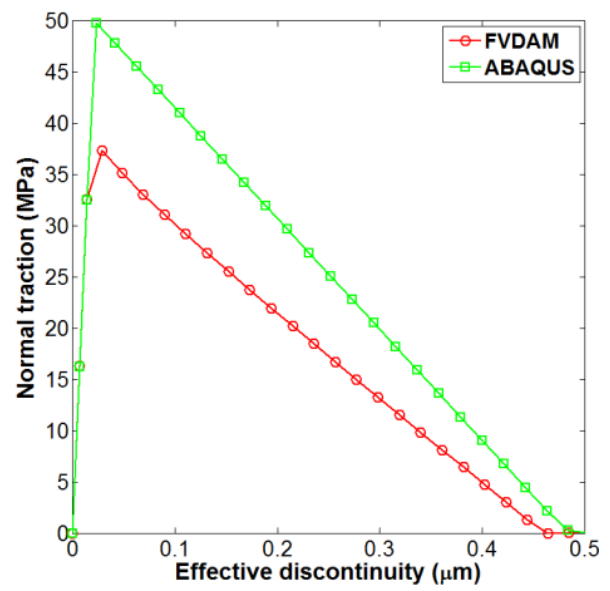

(e)

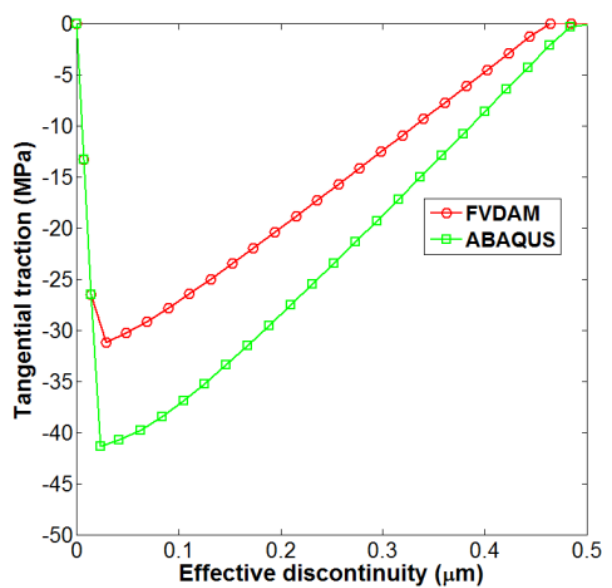

(f)

Figure 3.12 Effective traction (a, d), normal traction (b, e), tangential traction (c, f) VS effective displacement discontinuity for 1st interface (left) and 11th interface (right) under biaxial strain loading. 


\subsubsection{Transverse Shear Loading}

As indicated in Figure 3.13, FVDAM simulations predict that both the 1st and 11th interfaces separate completely at step 47, whereas Abaqus predicts complete separation of the 1st interface and 11th interfaces at steps 49 and 50, respectively. Figures 3.14(a, b, c) show the interfacial separation process for the 1st interface when the unit cell is under transverse shear. As expected the interface failure process is dominated by shear traction and interfacial behavior follows the bilinear relation along shear direction, along which the interface starts softening at 50MPa and separates completely at $0.46 \mu \mathrm{m}$. Excellent agreement is observed between FVDAM and Abaqus simulations, especially for the effective and normal traction responses, which are almost identical since the shear contribution is negligible. Figures 3.14(d, e, f) illustrate the interfacial separation process for the 11th interface, which is under combined normal and shear loading. Similar to Figures 3.12(d, e, f), when the interface is under combined stress state, damage initiation occurs at higher level of interfacial normal and tangential stresses. Hence to completely separate the interface, more energy needs to be dissipated in Abaqus simulation. Because of the stiffer interfacial behavior during the interfacial degradation stage, the corresponding homogenized response predicted by Abaqus is also slightly stiffer than the response generated by FVDAM, as shown in Figure 3.13.

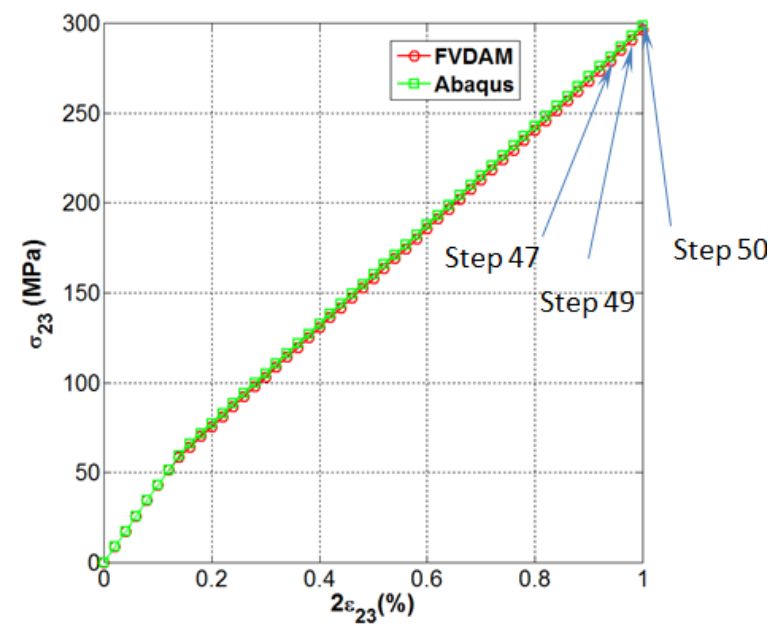

Figure 3.13 Homogenized response $\sigma_{23}$ and indication of loading steps at which the 1st and 11th interfaces separate completely. 


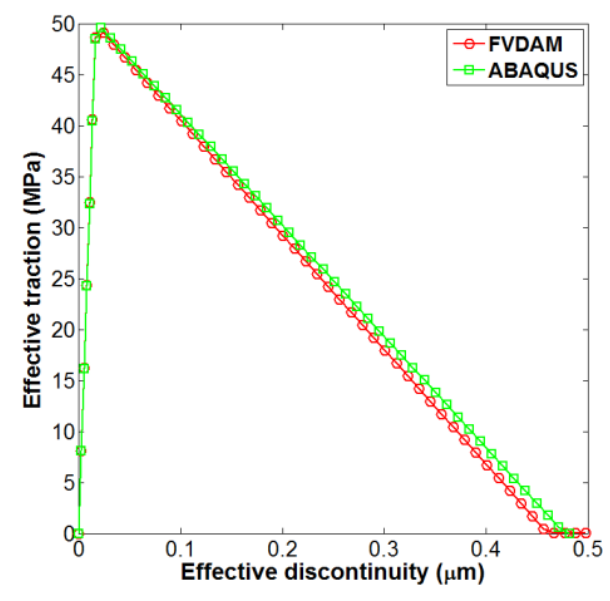

(a)

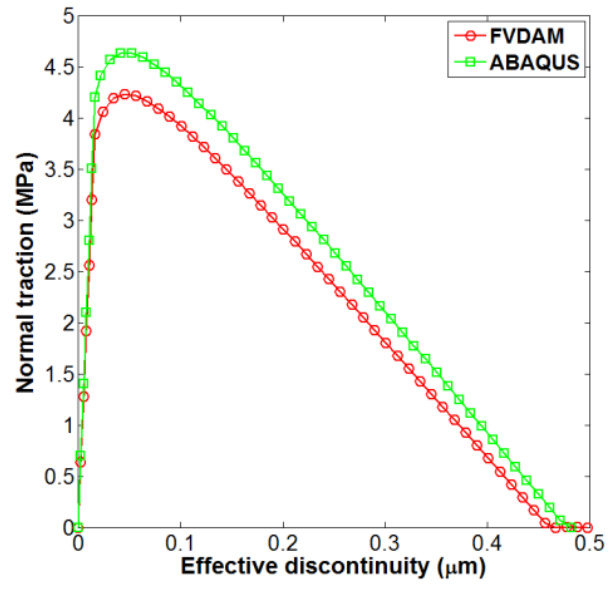

(b)

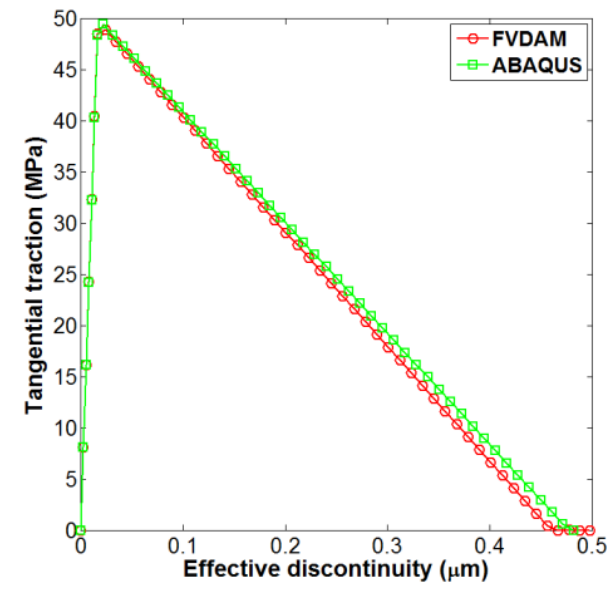

(c)

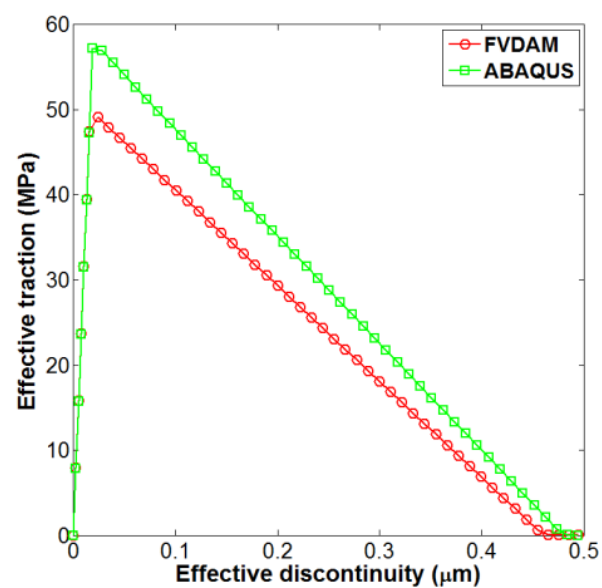

(d)

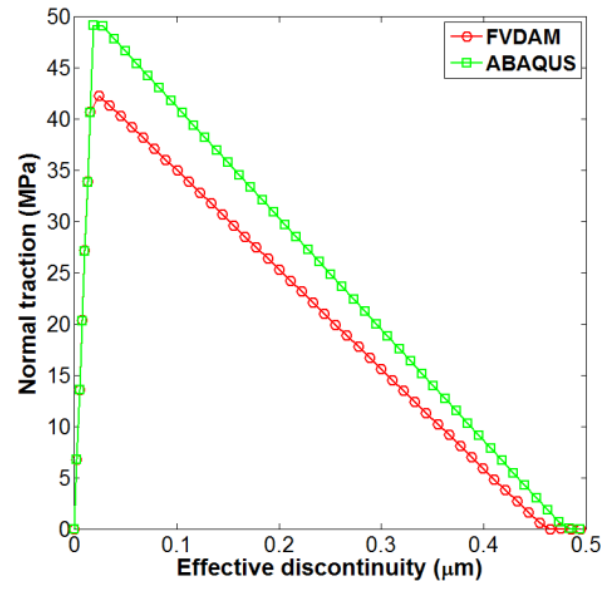

(e)

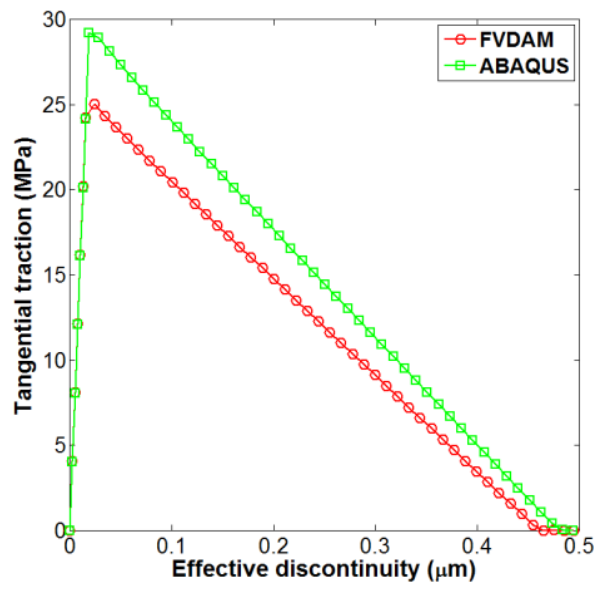

(f)

Figure 3.14 Effective traction (a, d), normal traction (b, e), tangential traction (c, f) VS effective displacement discontinuity for 1st interface (left) and 11th interface (right) under transverse shear loading. 


\subsection{Numerical Results under Bi-Axial Strain Loading}

From Section 3.3, it is known that when compression factor is 1e2, the homogenized responses, interfacial traction and displacement discontinuity and full-field stress distributions behave well. In the next two sections, comparison between CZM-FVDAM and Abaqus predictive capabilities in modeling interfacial debonding under bi-axial strain and transverse shear loading will be illustrated. For bi-axial strain simulations, the ratio of $-1 / 2$ between normal strains $\varepsilon_{33}$ and $\varepsilon_{22}$ is employed, and a compression factor of $1 \mathrm{e} 2$ is used in Abaqus simulations, unless explicitly stated otherwise.
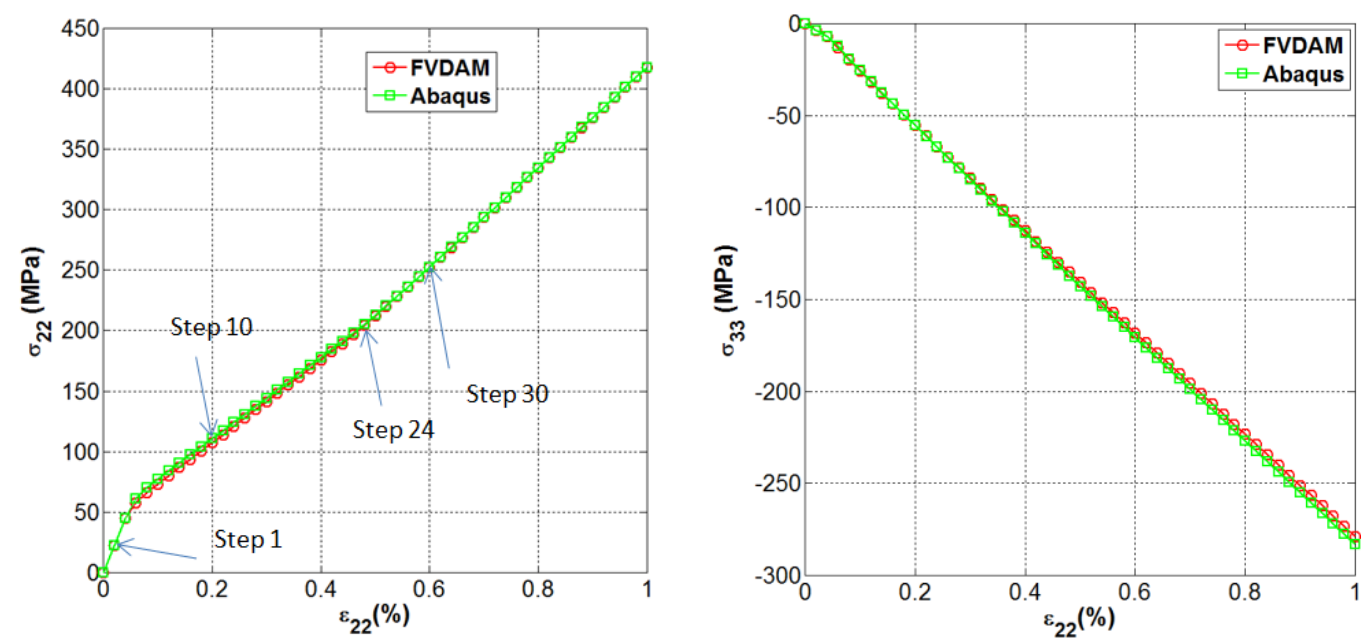

Figure 3.15 Selected homogenized responses in FVDAM and Abaqus under biaxial strain loading.

Figure 3.15 presents comparison of selected homogenized responses $\bar{\sigma}_{22}$ and $\bar{\sigma}_{33}$ along the $x_{2}$ and $x_{3}$ directions, illustrating very good agreement between FVDAM and Abaqus simulations. The homogenized normal response, $\bar{\sigma}_{22}$ vs $\bar{\varepsilon}_{22}$, can be generally divided into three stages. The first stage is linear which is characterized by elastic interfacial opening occurring between steps 1 and 2. The second stage is non-linear which involves interfacial degradation and debonding occurring between steps 3 and 29. The third stage is linear because of the arrest of interfacial cracking around the top and bottom regions of the fiber/matrix interface due to negative compressive normal stress. In the second stage of the non-linear homogenized response $\bar{\sigma}_{22}$ vs $\bar{\varepsilon}_{22}$, Abaqus' prediction is slightly stiffer than the FVDAM's because more energy needs to be dissipated in Abaqus simulation when the interface is under combined normal and shear tractions. This was already seen in Figures 3.12(d, e, f) and 3.14(d, e, f), in 
which both normal and tangential tractions predicted by Abaqus simulations were always higher relative to FVDAM.
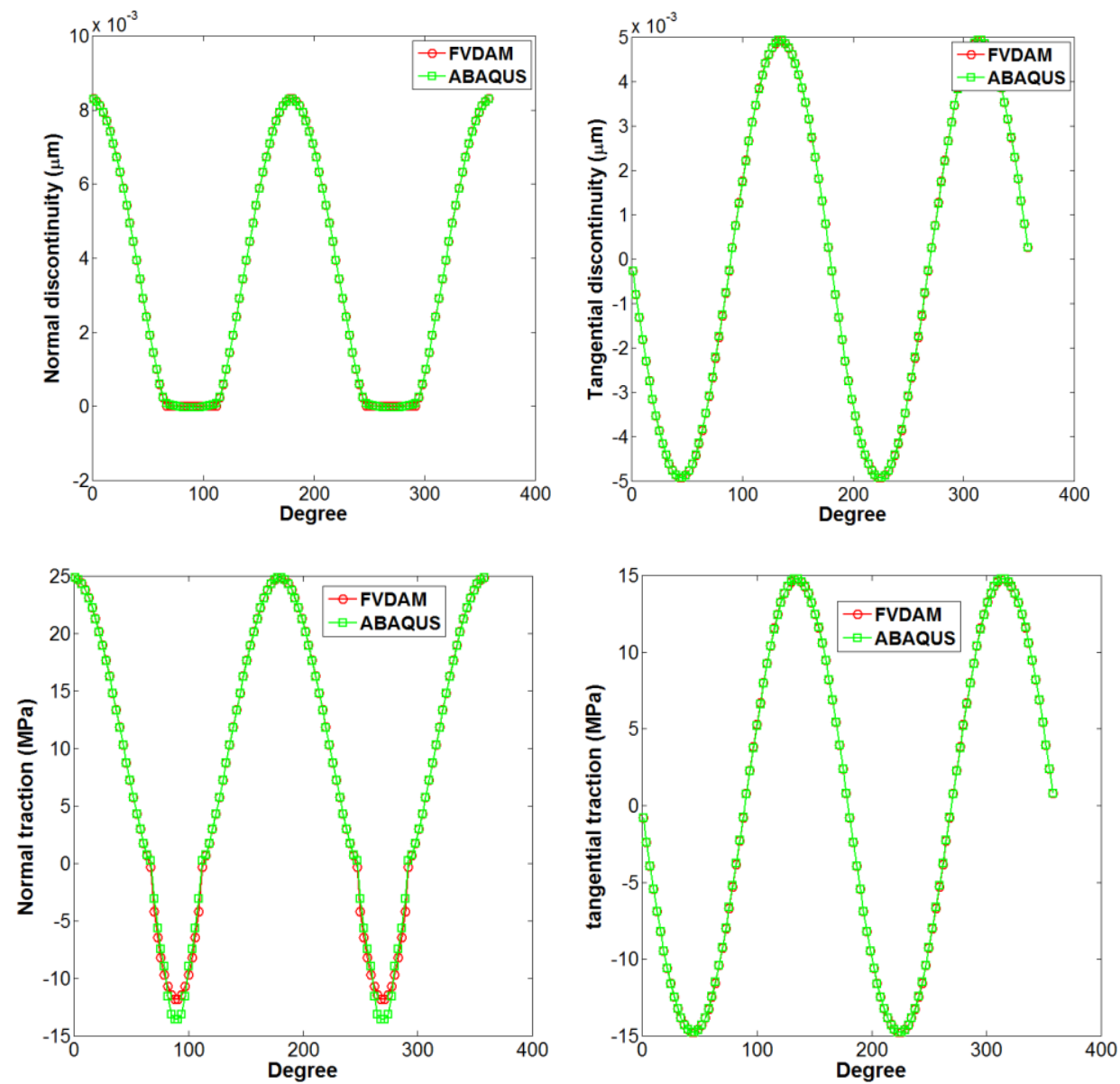

Figure 3.16 Interfacial traction and displacement discontinuity distributions in FVDAM and Abaqus at step 1.

Figure 3.16 shows the interfacial traction and displacement discontinuity distributions predicted by FVDAM and Abaqus at step 1, which is characterized by elastic interfacial behavior. The FVDAM and Abaqus predictions have excellent agreement except for the slight difference in normal traction around the top and bottom regions of the fiber/matrix interface. Figure 3.17 shows the corresponding interfacial traction and displacement discontinuity distributions at step 10, where interfaces at, and in the vicinity of, $0^{\circ}$ and $180^{\circ}$ regions are experiencing interfacial degradation. Generally, very good agreement between the two sets of predictions is observed, especially for displacement discontinuity distributions, with some differences for the interfaces around $0^{\circ}$ and $180^{\circ}$ locations which are experiencing degradation. Tractions predicted by Abaqus are slightly higher than those predicted by FVDAM because of 
the stiffer response of traction-separation relation for damage evolution, which was illustrated in Figures 3.12(d, e, f) and 3.14(d, e, f).
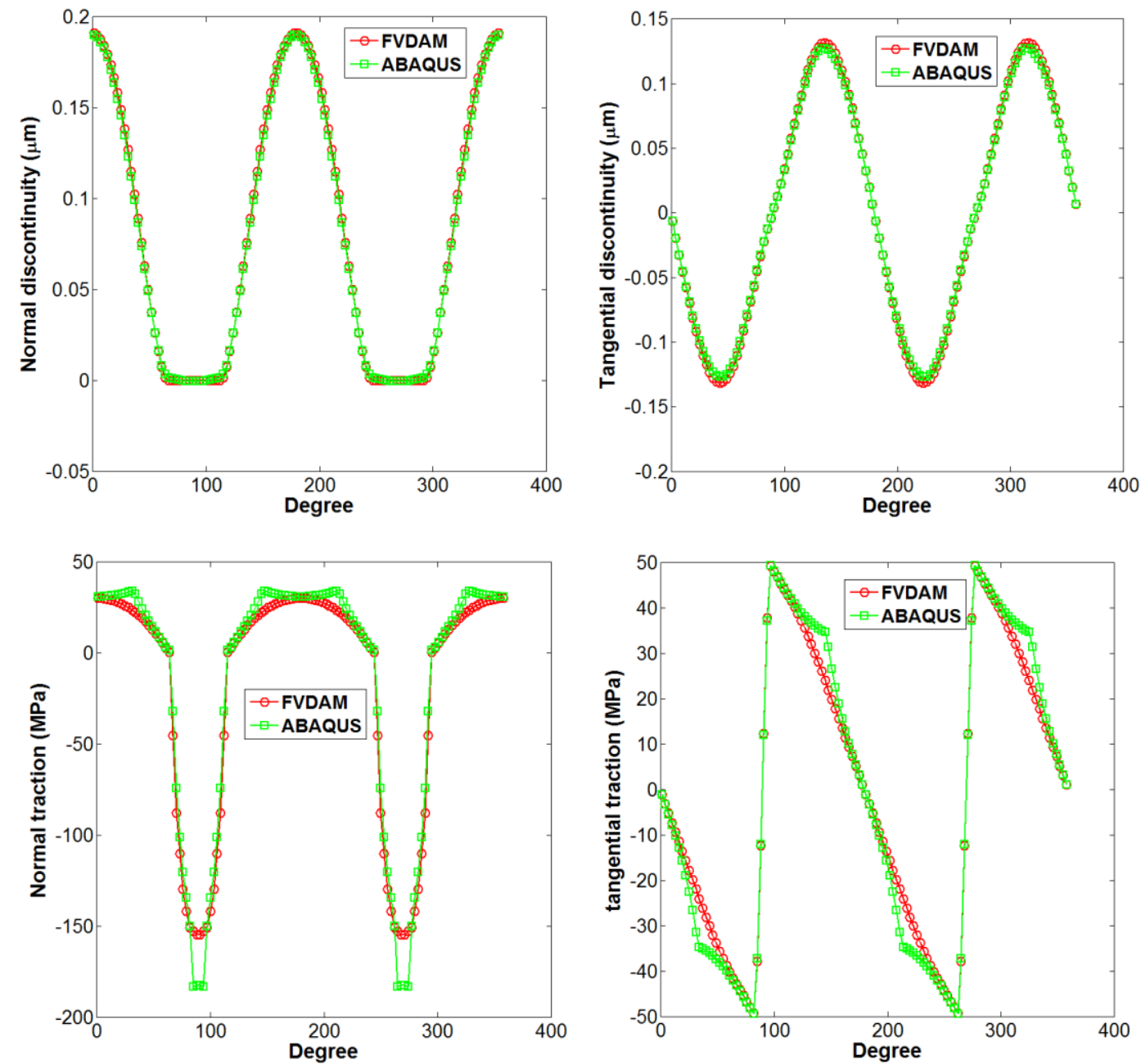

Figure 3.17 Interfacial traction and displacement discontinuity distributions in FVDAM and Abaqus at step 10.

Figure 3.18 illustrates interfacial traction and displacement discontinuity distributions predicted by FVDAM and Abaqus simulations at step 30, at which interfacial debonding is arrested around the angle of $\pm 65^{\circ}$. Normal and shear displacement discontinuity distributions and tangential traction distributions are almost identical. For normal traction, the difference is located around top and bottom regions of the fiber/matrix interface, where interfaces are under compressive tractions, with the compressive tractions predicted by Abaqus simulations higher than the corresponding tractions predicted by FVDAM. If a higher compression factor is used in Abaquis simulations then higher compressive tractions can be expected. 

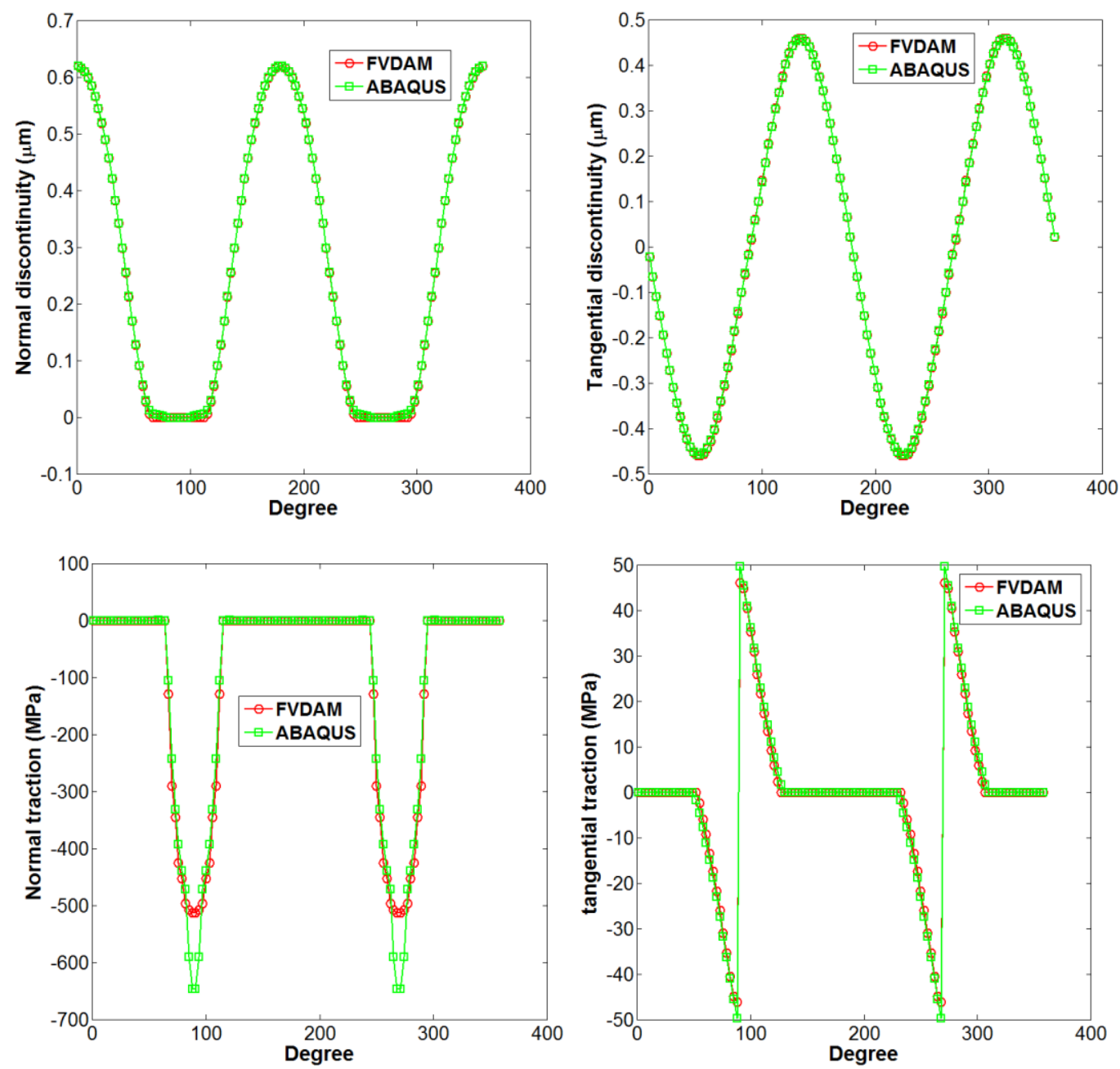

Figure 3.18 Interfacial traction and displacement discontinuity distributions in FVDAM and Abaqus at step 30.

Figures 3.19-3.21 illustrate in-plane stress evolution at steps 1, 10 and 30 predicted by FVDAM and Abaqus simulations, with excellent agreement observed for all steps. With the development of interfacial degradation and debonding, normal stress $\sigma_{22}$ and shear stress $\tau_{23}$ decrease along the left and right hand side of the fiber/matrix interface. This decrease is accompanied by dramatic evolution of stress concentrations around the top and bottom regions of the fiber/matrix interface because of the loss of load carrying capability in the center portion of the fiber/matrix interface. 


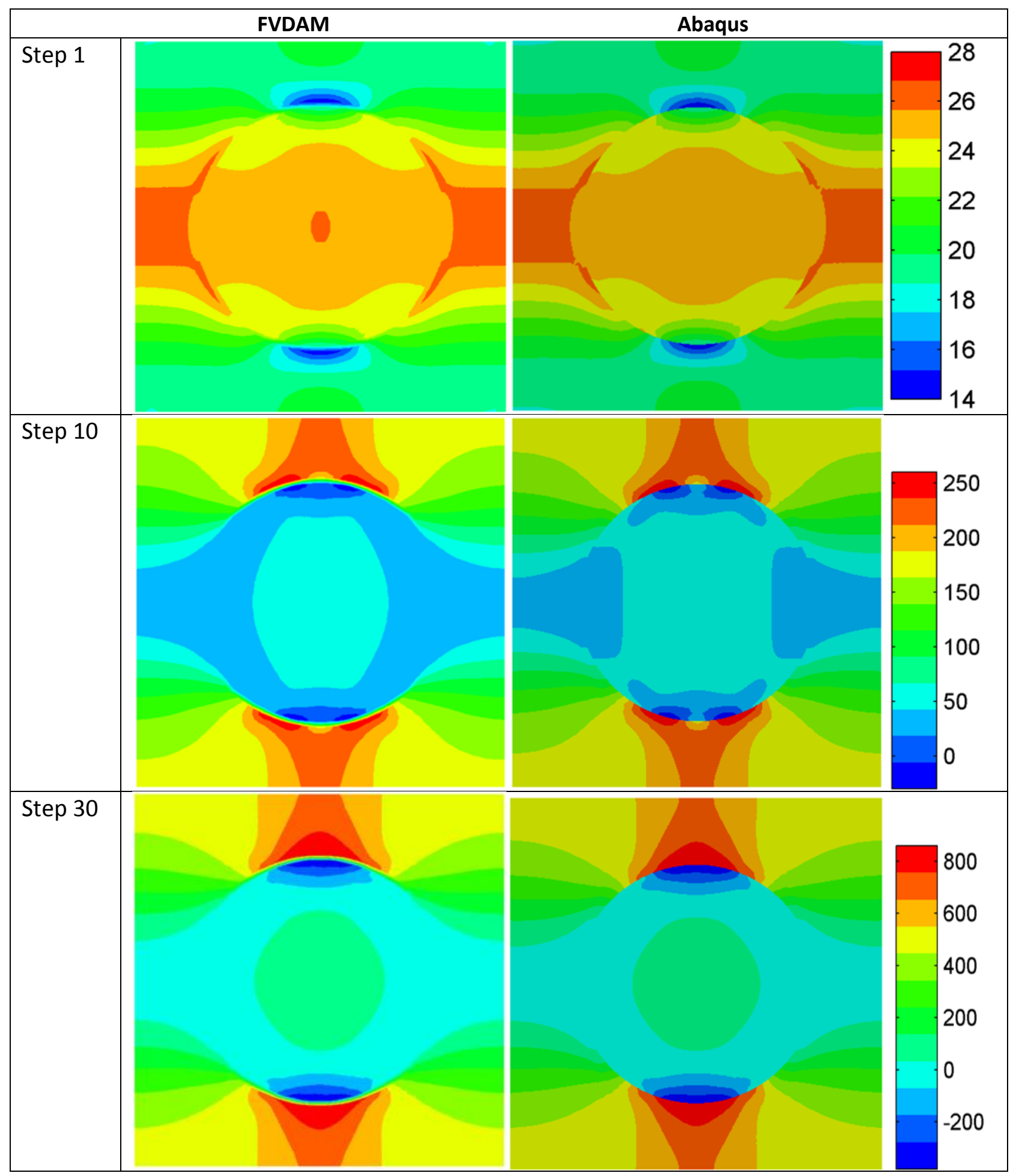

Figure $3.19 \sigma_{22}$ distributions in FVDAM and Abaqus at difference steps. 


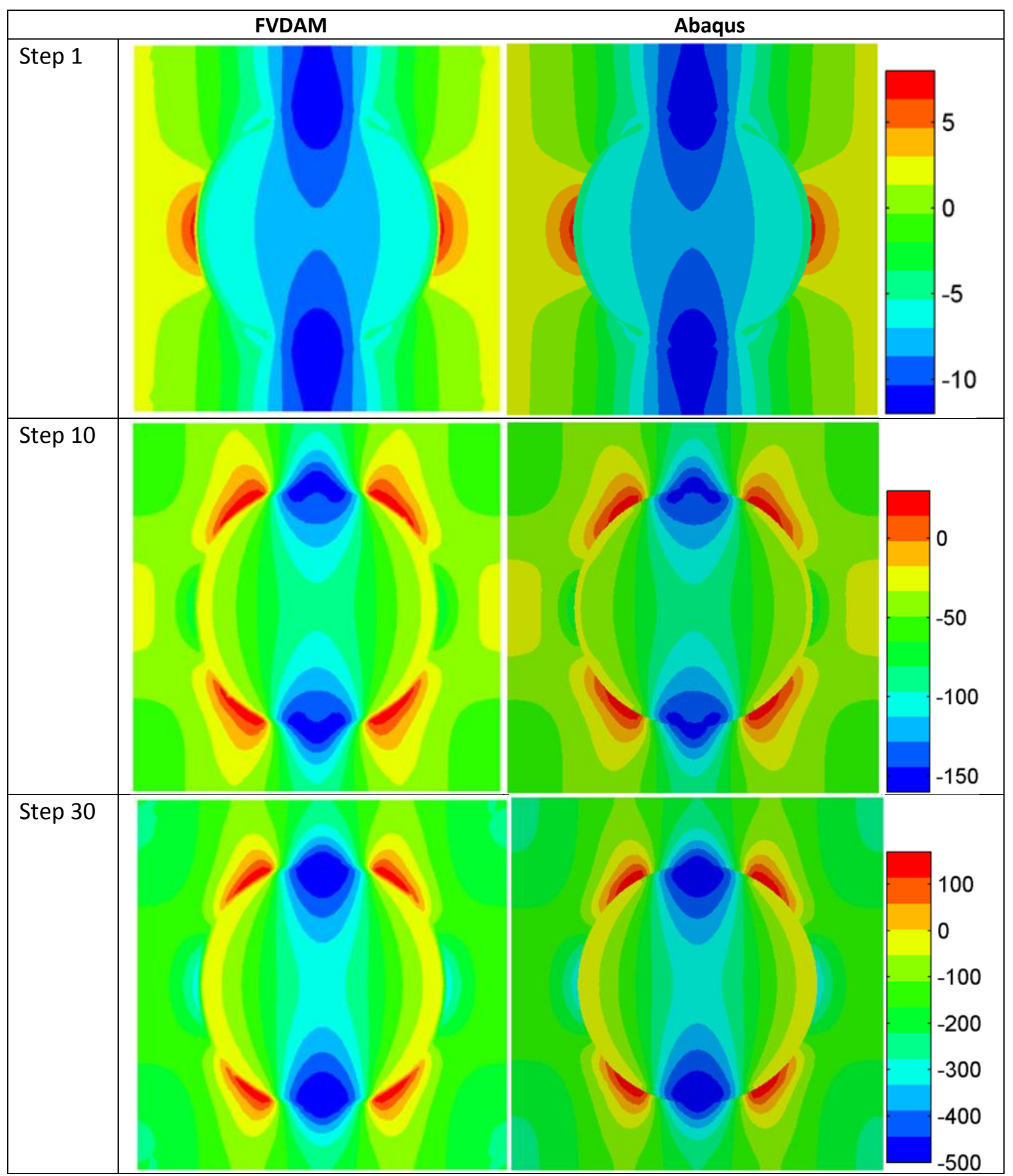

Figure $3.20 \sigma_{33}$ distributions in FVDAM and Abaqus at difference steps. 


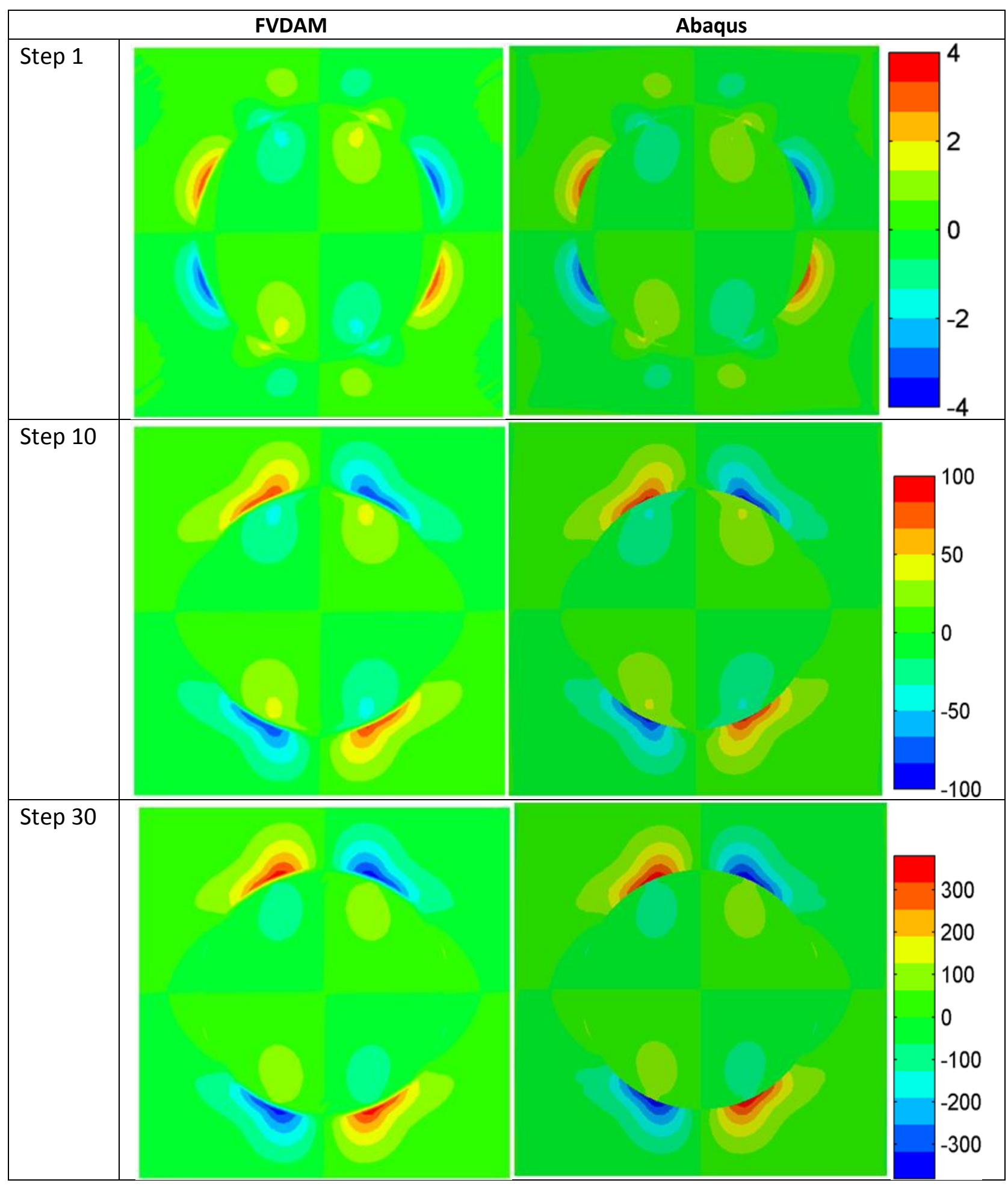

Figure $3.21 \sigma_{23}$ distributions in FVDAM and Abaqus at difference steps. 


\subsection{Numerical Results under Transverse Shear}

In this section, detailed comparison between FVDAM and Abaqus is conducted under transverse shear strain loading, including unit cell homogenized responses, interfacial traction and displacement distributions and full-field stress distributions. Figure 3.22 shows the selected homogenized responses of $\bar{\sigma}_{22}$ and $\bar{\sigma}_{23}$, noting that $\bar{\sigma}_{22}$ has the same response as $\bar{\sigma}_{33}$. The responses predicted by FVDAM and Abaqus simulations are very similar, with the $\bar{\sigma}_{23}$ response being slightly stiffer because in Abaqus simulations the interface under combined tensile and shear loading is more difficult to fail as shown in Figures 3.12(d, e, f) and 3.14(d, e, f), leading to higher normal and tangential tractions during the interfacial degradation stage.
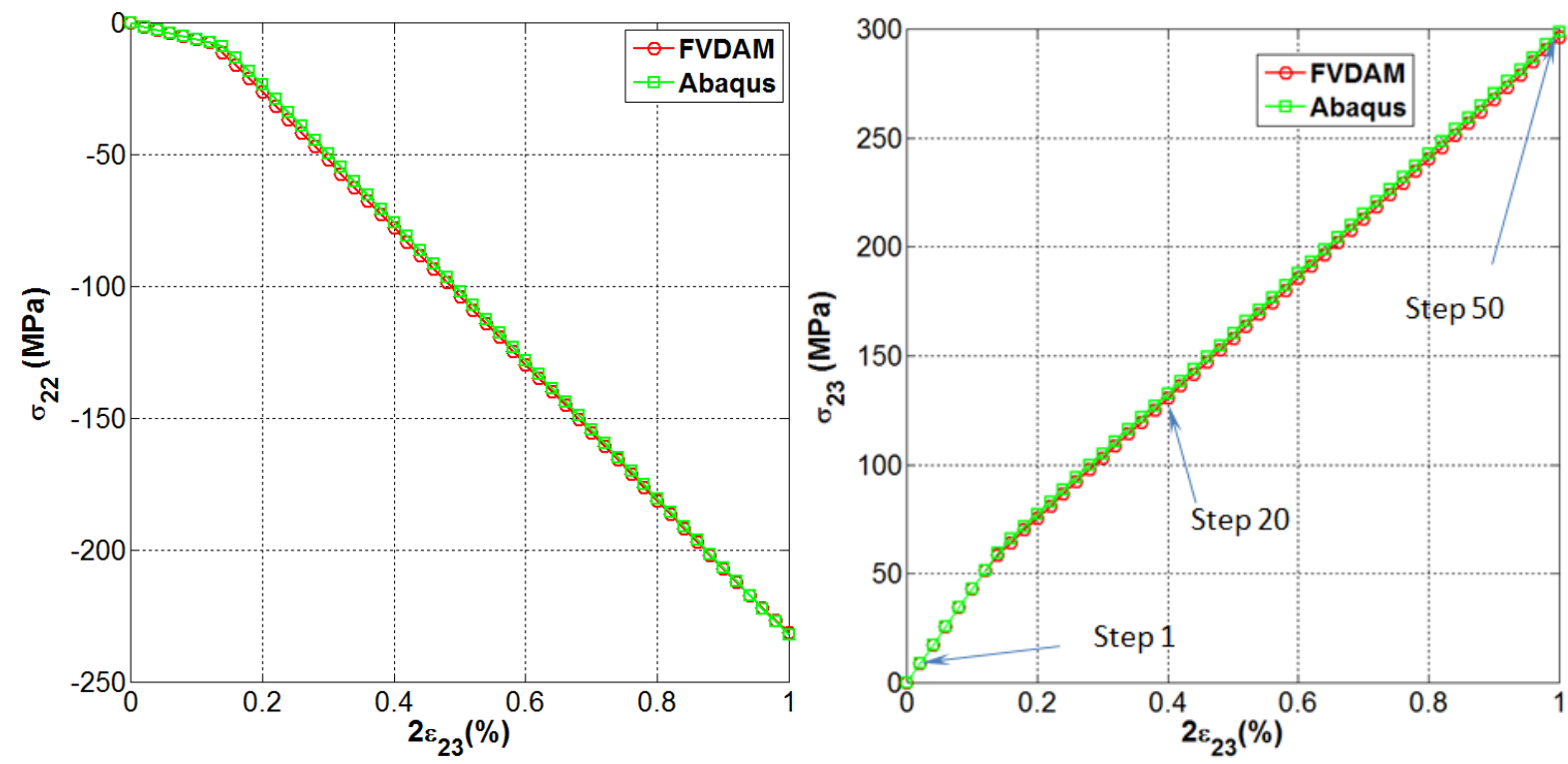

Figure 3.22 Selected homogenized responses with FVDAM and Abaqus under transverse shear.

Figures 3.23-3.25 present comparisons of the interfacial traction and displacement discontinuity distributions predicted by FVDAM and Abaqus simulations at step 1 with the interface responding elastically, at step 20 with the interface experiencing degradation, and at step 50 when the interface is debonded. Generally, results from FVDAM and Abaqus simulations exhibit very good agreement at all steps, with some slight differences. Specifically, in Figure 3.23, interfacial normal tractions around $135^{\circ}$ and $315^{\circ}$ locations are slightly higher in Abaqus predictions at step 1 due to high penalty stiffness used to resist interpenetration, in 
contrast with the direct enforcement of traction and displacement continuity conditions in FVDAM.
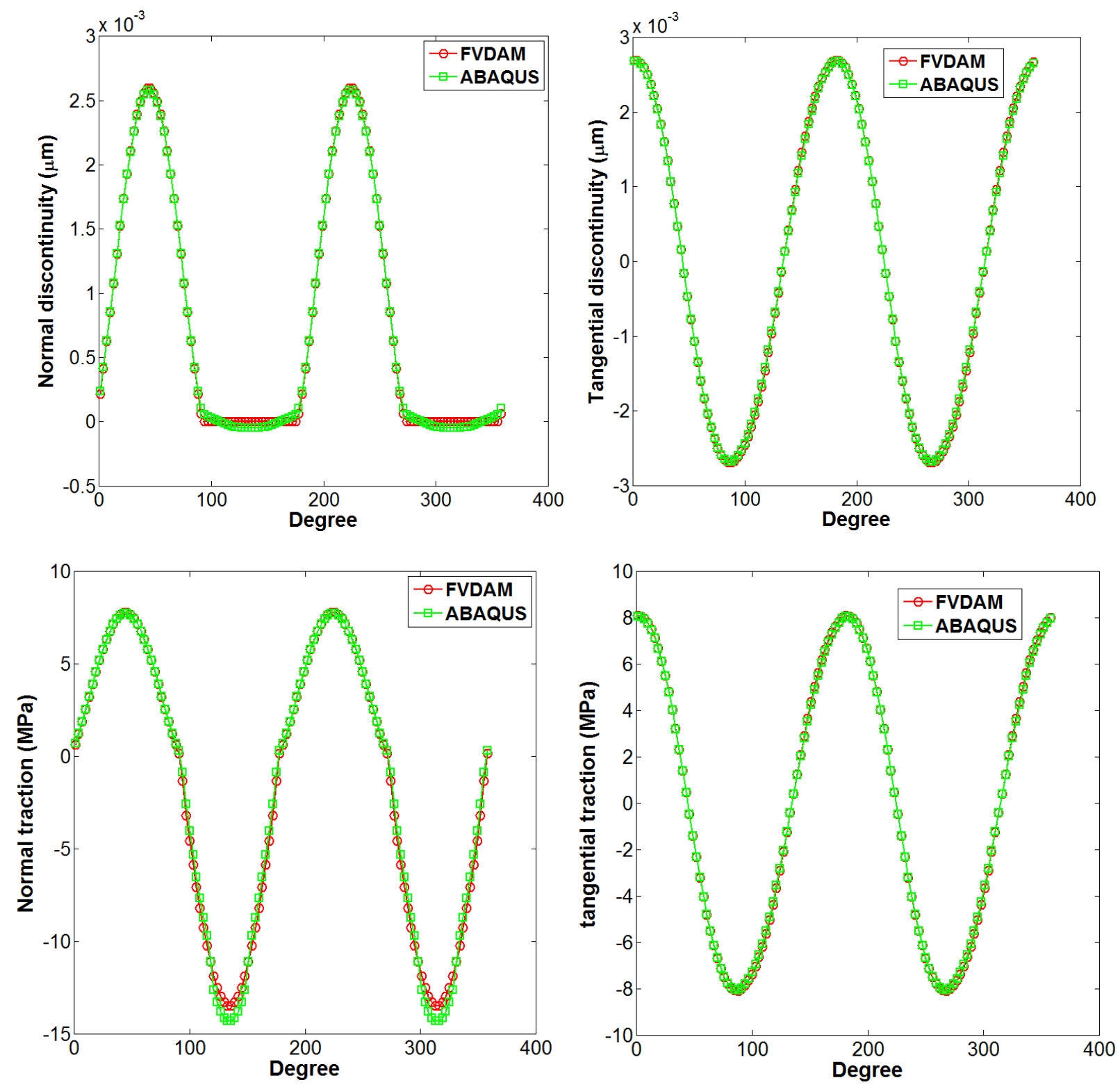

Figure 3.23 Interfacial traction and displacement discontinuity distributions in FVDAM and Abaqus at step 1.

Figure 3.24 illustrates the interfacial degradation responses predicted by FVDAM and Abaqus simulations at step 20, with the degradation initially occurring around $45^{\circ}$ and $225^{\circ}$ and propagating away from those two locations. For the degraded interfaces, both normal and tangential tractions are higher in Abaqus than FVDAM predictions because of the stiffer response during the damage evolution stage seen in Figures 3.12(d, e, f) and 3.14(d, e, f) when 
the interfaces are under combined normal and tangential tractions. Figure 3.25 illustrates the extensive interfacial debonding in the range of $\left[0^{\circ} 100^{\circ}\right]$ and $\left[180^{\circ} 280^{\circ}\right]$ at step 50 . Outside of these ranges, debonding is arrested due to compressive normal tractions. FVDAM and Abaqus produce the same predictions.
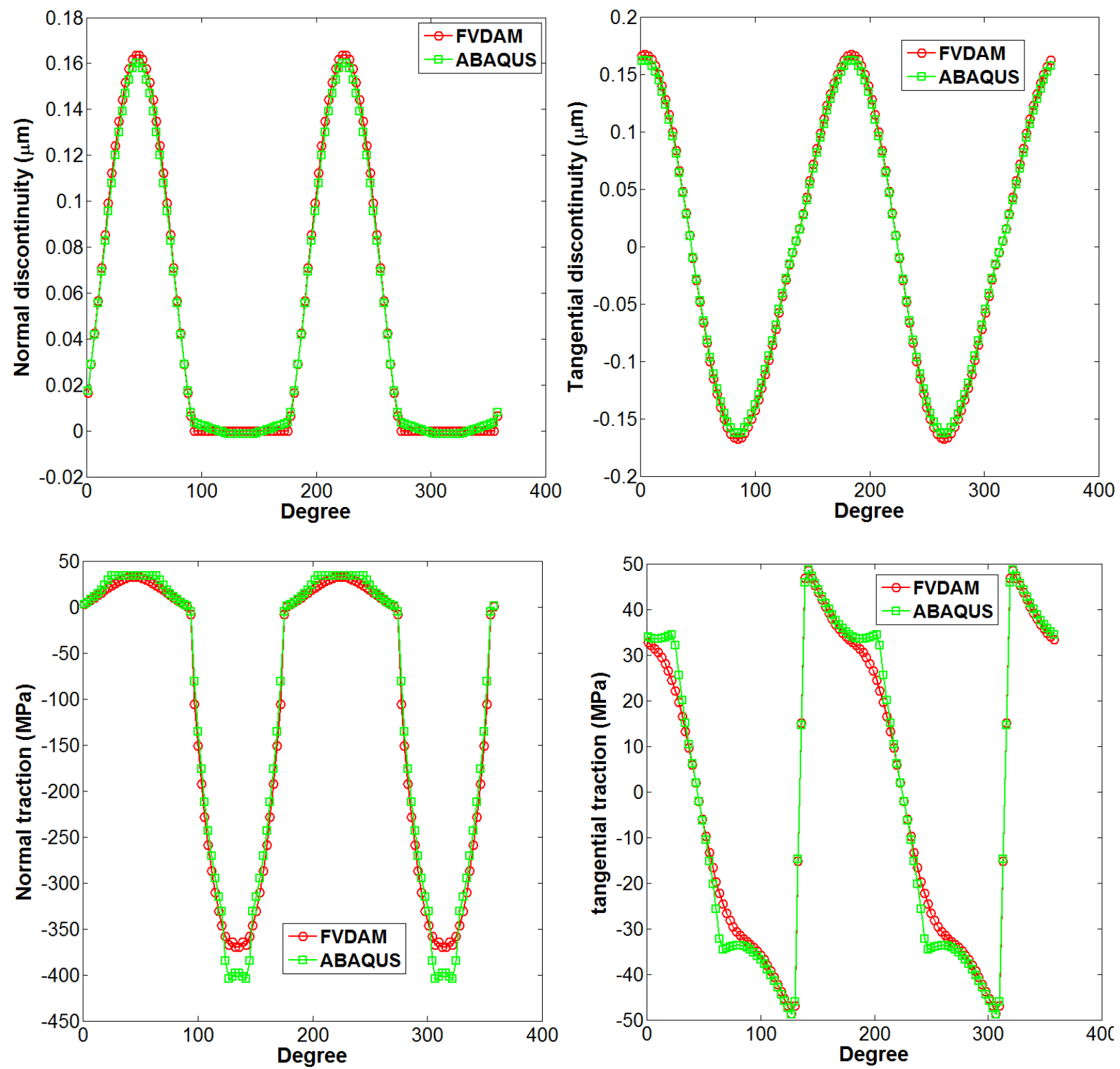

Figure 3.24 Interfacial traction and displacement discontinuity distributions in FVDAM and Abaqus at step 20. 

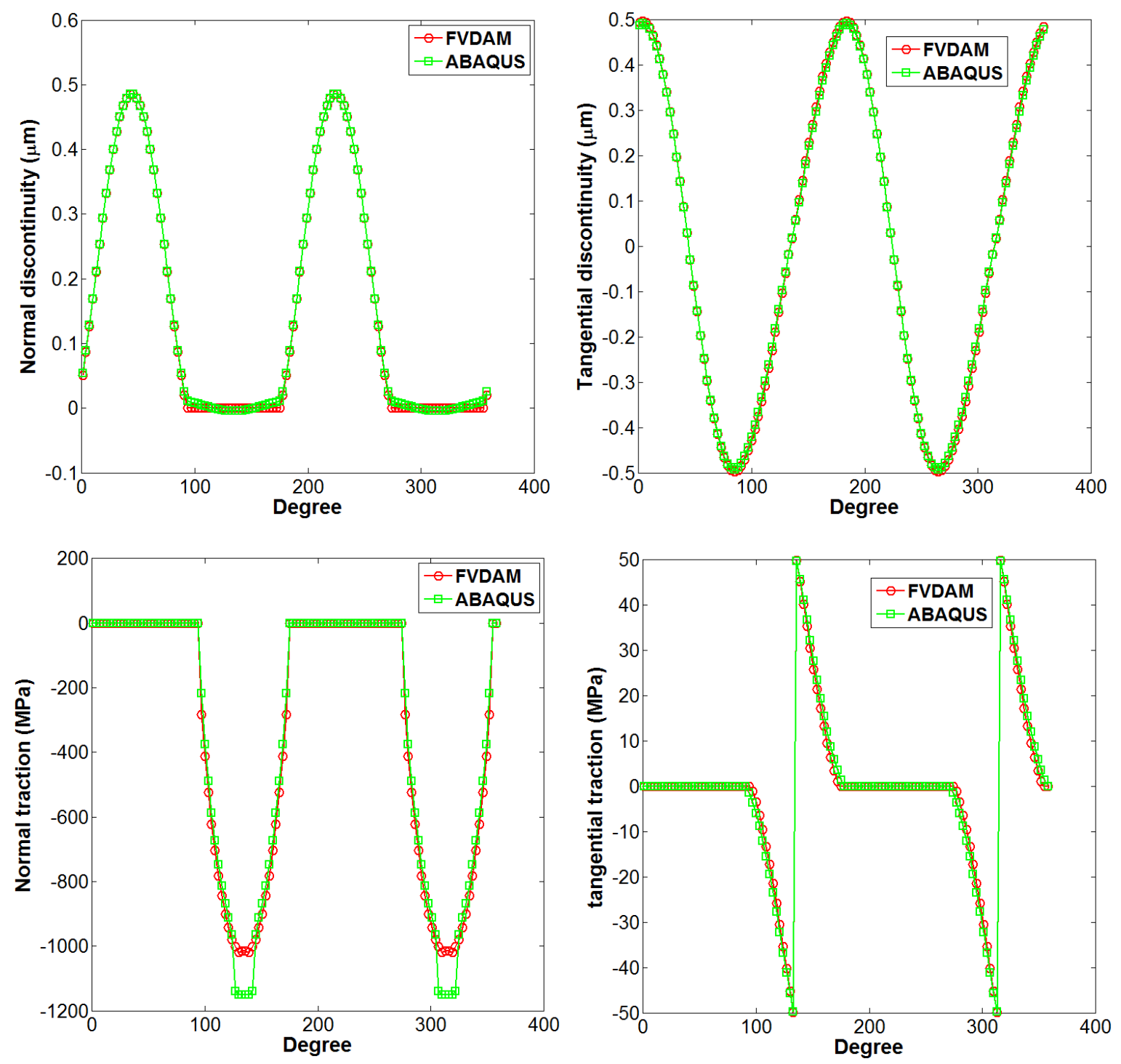

Figure 3.25 Interfacial traction and displacement discontinuity distributions in FVDAM and Abaqus at step 50.

Figures 3.26-3.28 present full-field stress distributions at steps 1, 20 and 50, respectively, illustrating that excellent agreement is observed between FVDAM and Abaqus predictions. Particularly, by looking at the evolution of the transverse shear stress $\sigma_{23}$, the interfacial debonding process can be understood transparently, with interfacial damage initiating at two angular orientations of $45^{\circ}$ and $225^{\circ}$ and then propagating away from these two locations. 


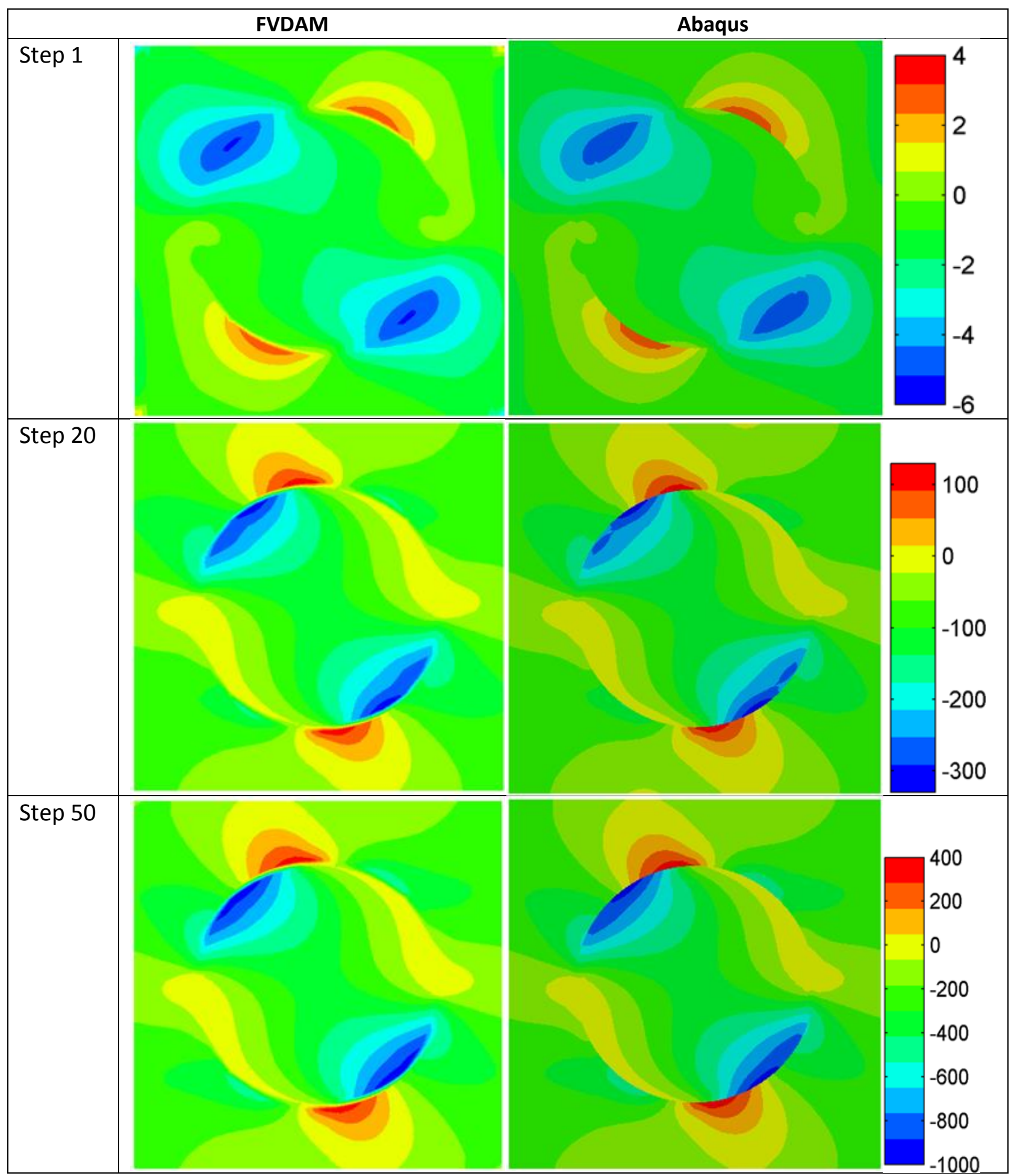

Figure $3.26 \sigma_{22}$ distributions in FVDAM and Abaqus at difference steps. 


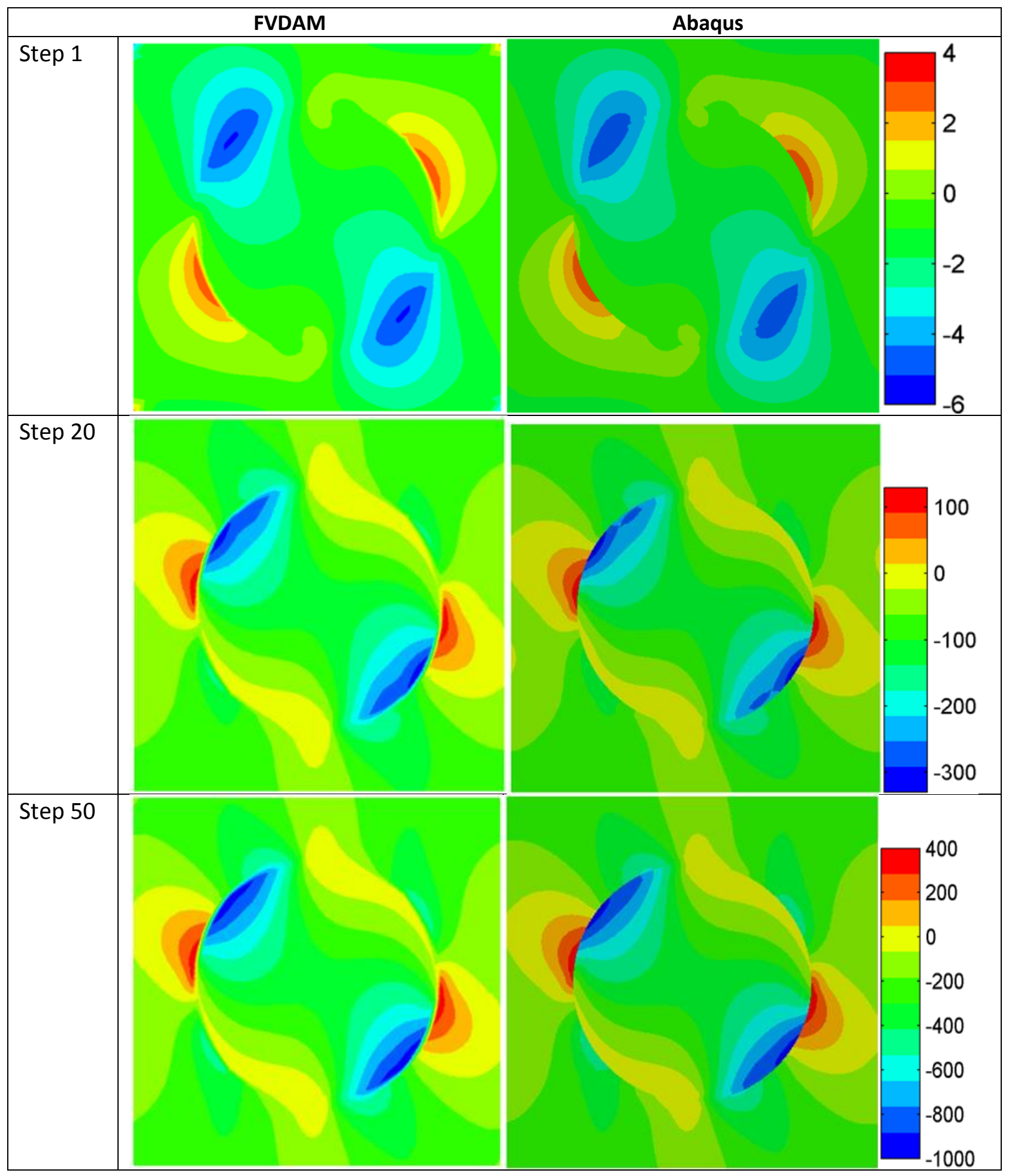

Figure $3.27 \sigma_{33}$ distributions in FVDAM and Abaqus at difference steps. 


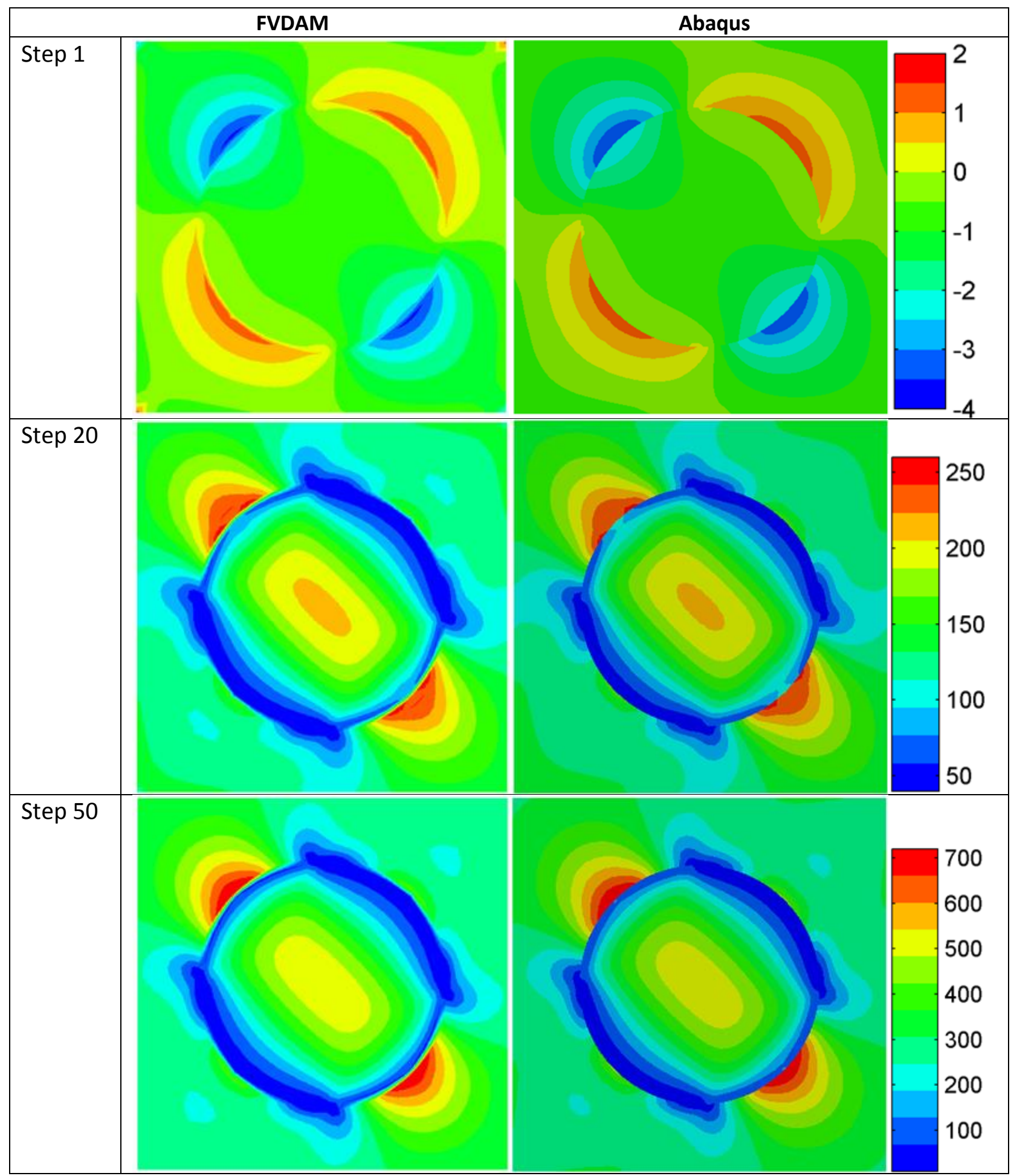

Figure $3.28 \sigma_{23}$ distributions in FVDAM and Abaqus at difference steps. 


\subsection{Effect of Fiber/Matrix Modulus Contrast}

From previous study, it is known that for the $\mathrm{SiC} / \mathrm{Ti}$ material system when the elastic interfacial stiffness $3 \times 10^{3} \mathrm{MPa} / \mu \mathrm{m}$ and compression factor 100 are used, Abaqus is able to complete the analysis without convergence issues, and the interfacial separation process can be captured correctly for both bi-axial and transverse shear loadings. Also, very good agreement between Abaqus and FVDAM predictions is obtained for the unit cell homogenized response, interfacial separation, and full-field stress distribution. In these simulations, the Young's modulus ratio between fiber and matrix was 4.3. Figure 3.29 compares the homogenized response of SiC/Ti predicted by Abaqus and FVDAM simulations with the homogenized response of a unidirectional composite with a soft fiber whose Young's modulus is $1 / 2$ of the titanium matrix and the Poisson's ratio is the same as that of the $\mathrm{SiC}$ fiber. As observed in the figure, the Abaqus simulation terminates at the load level of $0.4 \%$ because of convergence issues. On the other hand, if a compression factor of 10 instead of 100 is used for the system, as shown in Figure 3.30, Abaqus can complete the analysis without convergence issues. Hence the compression factor is affected by the fiber/matrix modulus mismatch and parametric study needs to be conducted before specification of the factor for a particular material system.
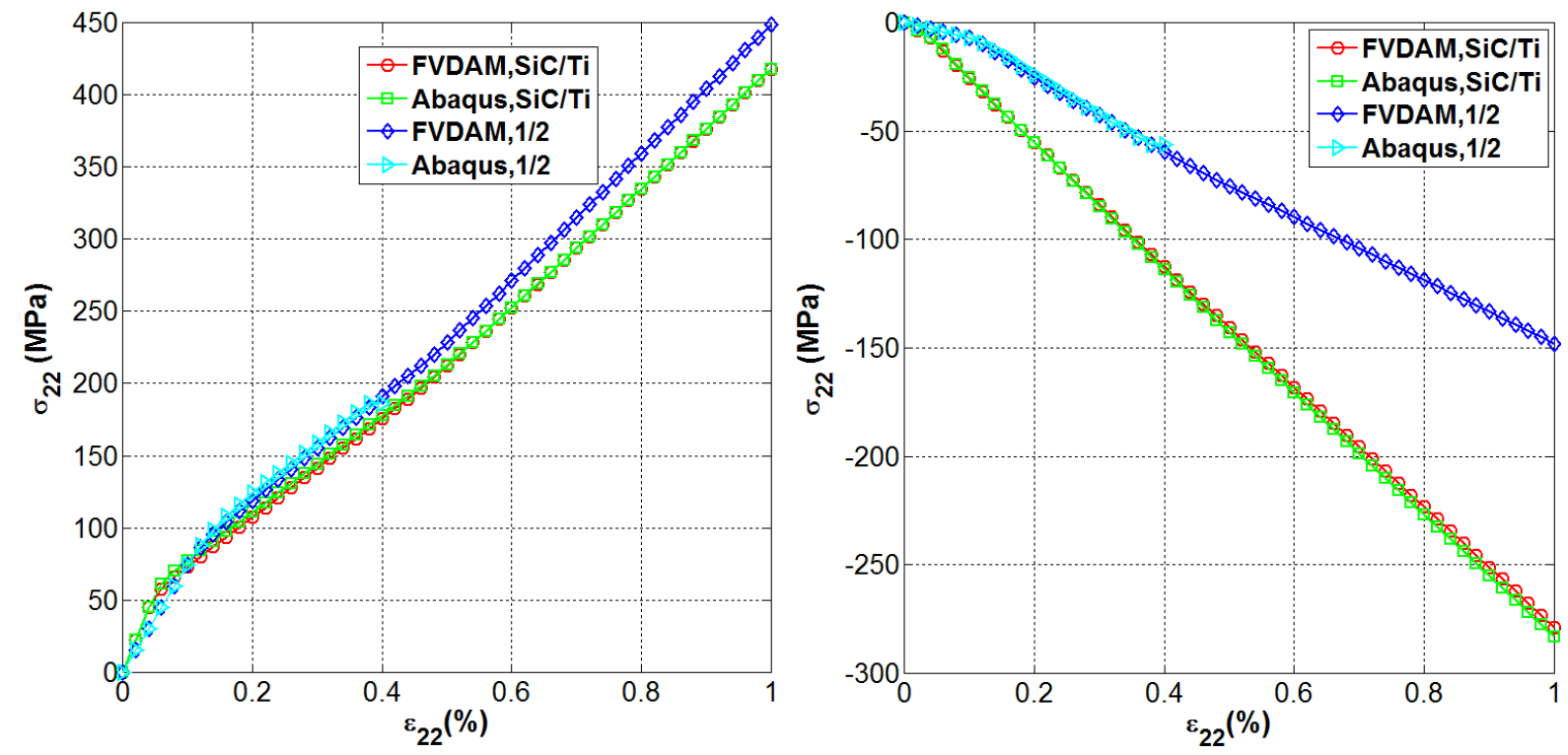

Figure 3.29 Homogenized response predicted by FVDAM and Abaqus when compression factor of 100 is used in Abaqus simulations. 

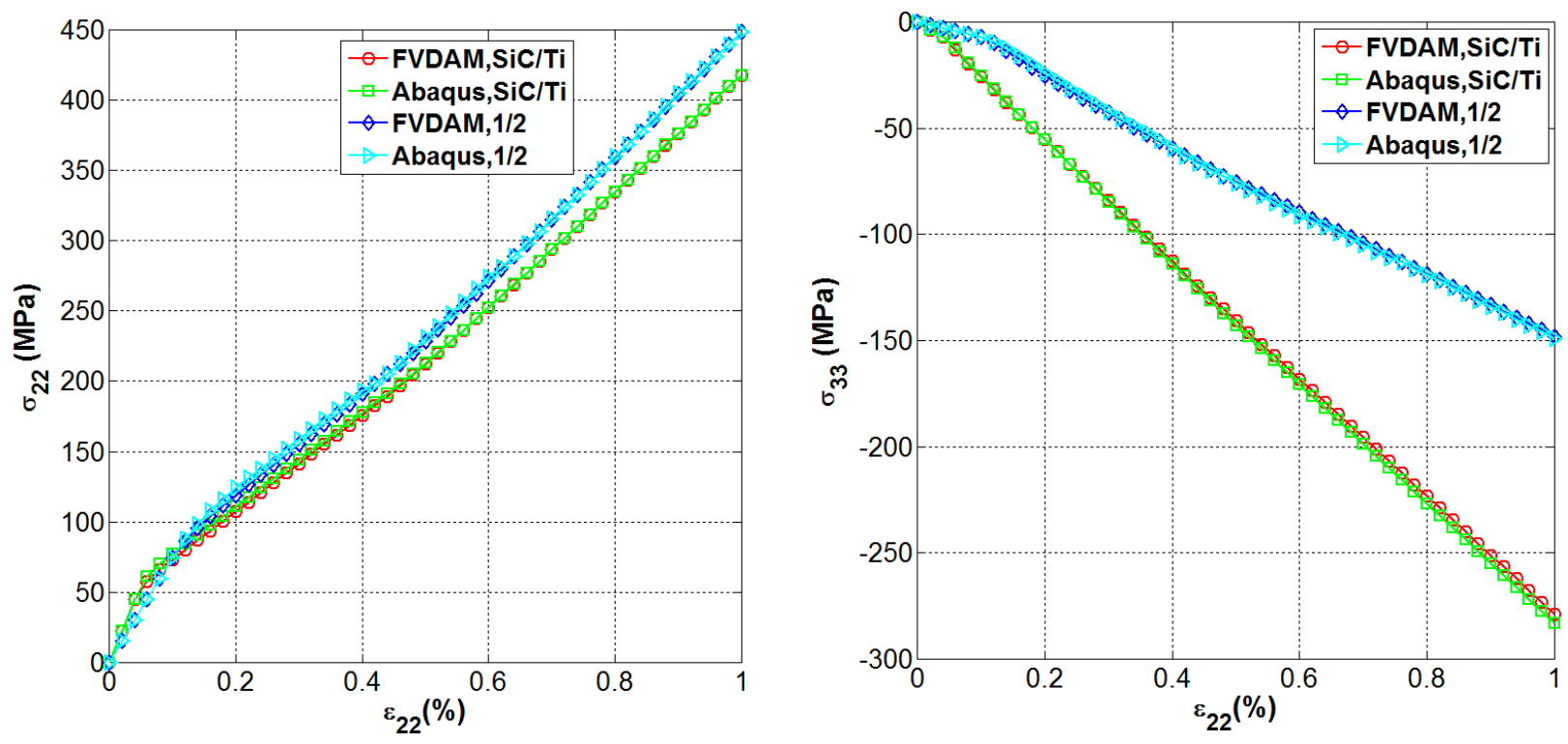

Figure 3.30 Homogenized response of FVDAM and Abaqus when compression factor of 10 is used in Abaqus simulations.

\subsection{Summary and Discussion}

FVDAM's and Abaqus's capability in predicting interfacial debonding was critically assessed under biaxial strain and transverse shear loading. Excellent agreement was obtained between the predictions of both approaches for the homogenized responses, interfacial quantity distributions and stress field distributions provided that a suitable compression factor is chosen for Abaqus. This factor should be high enough to resist interfacial interpenetration when the interface is under normal compressive stress, but cannot be excessively high in order to avoid numerical problems. In addition, suitable compression factor needs to be identified for different fiber/matrix modulus mismatch rather than using the same number. In contrast, the interpenetration issue does not exist in FVDAM simulations because the interfacial tractionseparation relation in the normal direction is replaced by traction and displacement continuity conditions directly once the interface is under compressive normal traction. This is efficiently accomplished by eliminating corresponding equations in the global auxiliary system of equations without re-assembling the primary global system of equations.

When an interface is under pure tensile traction or shear traction, the cohesive law in FVDAM and Abaqus behave exactly the same and follows the bilinear separation relation. When an interface is under combined normal and tangential tractions, the interfacial damage evolves differently. Damage initiation in FVDAM is determined by the effective traction whereas 
damage initiation in Abaqus is determined by either the maximal normal or tangential traction. In Abaqus, damage evolution failure process dissipates more energy than in FVDAM and this is also reflected by the slightly stiffer homogenized responses in the non-linear stage.

In FVDAM, periodicity boundary conditions are directly incorporated into the theory and the desired loading types can be easily applied by adjusting average strains via homogenized Hook's law. In Abaqus, periodicity boundary conditions need to be applied carefully by correctly constraining the nodal displacements along boundary pairs of the unit cell. Another difference between these two approaches is how the analysis is conducted when the unit cell is under outof-plane shear loading. In FVDAM the analysis employs the same 2D discretization as that used in this chapter with an even smaller system of equations than under in-plane loading. FVDAM simulation results under combined in-plane and out-of-plane loading will be presented in Chapter 7 using 2D discretization of a unit cell. In contrast, the same problem analyzed in Abaqus requires $3 \mathrm{D}$ simulation which is much more costly with regard to computational efficiency. 


\section{Chapter 4}

\section{Debonding in SiC/Ti Composites}

\subsection{Introduction}

To demonstrate the capability of the implemented CZM within the FVDAM framework in simulating progressive interfacial damage evolution leading to phase separation in heterogeneous materials, we revisit the classic SiC/Ti debonding problem. Unidirectionally-reinforced $\mathrm{SiC} / \mathrm{Ti}$ composites were first proposed in the 1990's for use in aircraft engine turbine blades to increase the engine's operating temperature, and hence efficiency, by reducing creep. However, the high consolidation temperature of the $\mathrm{SiC} / \mathrm{Ti}$ material system produces a chemical reaction at the fiber/matrix interface which results in substantially degraded chemical bond, leading to premature fiber/matrix interfacial debonding at low transverse normal stresses. Hence this system is a good candidate to validate the predictive capability of the CZM-based FVDAM theory relative to the available experimental data reported by Johnson et al. (1990).

In section 4.2, the response of the $\mathrm{SiC} / \mathrm{Ti}$ composite under transverse loading based on a square unit cell architecture is simulated immediately after the fabrication cooldown when the fiber/matrix interface is presumed intact. In section 4.3, the subsequent response after initial preload cycles is simulated and the parametric study is carried out to determine the extent of the damage caused by initial preload cycles. During the fabrication process, local fiber arrangement may be disturbed, resulting in regions with a mixture of square and hexagonal fiber distributions. Hence the effects of unit cell architecture and loading direction on interfacial damage evolution and homogenized response are also investigated in sections 4.4 and 4.5 , respectively. The effect of loading direction on interfacial damage and homogenized response is limited to hexagonal unit cells because of the loss of transverse isotropy in the inelastic region for this material architecture. 


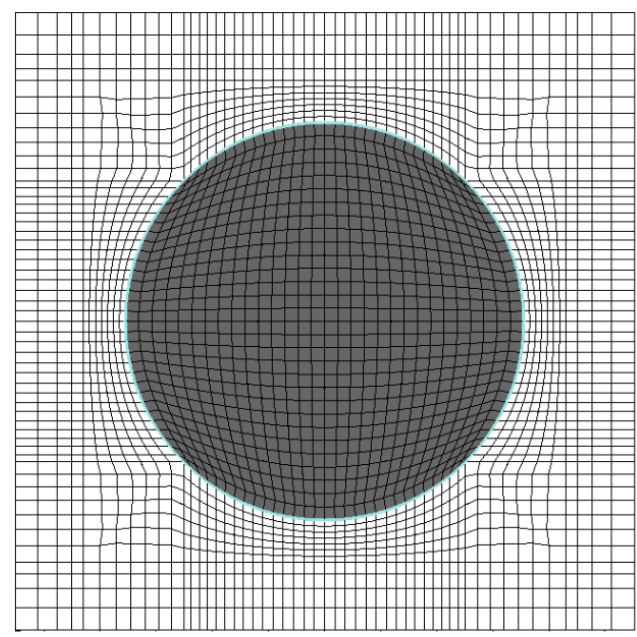

Figure 4.1 Unit cell geometry and discretization containing 0.325 fiber volume fraction.

Table 4.1 Thermoelastic properties of the SiC fibers and Ti matrix.

\begin{tabular}{|c|c|c|c|c|}
\hline Material & $E(\mathrm{GPa})$ & $v$ & $\alpha\left(\mu /{ }^{\circ} \mathrm{C}\right)$ & $\sigma_{y}(\mathrm{MPa})$ \\
\hline $\mathrm{SCS}_{6}$ & 400 & 0.25 & 4.86 & --- \\
\hline $\mathrm{Ti}-15$ & 92.38 & 0.35 & 9.72 & 689.5 \\
\hline
\end{tabular}

The unit cell representative of the unidirectional SiC/Ti composite in the cross section normal to the fiber direction and its discretization into $52 \times 52$ subvolumes is shown in Figure 4.1 with the highlighted cohesive zone around the entire fiber. The $\mathrm{SiC}$ fiber volume fraction is 0.325 , the diameter of the fiber is $142 \mu \mathrm{m}$ and the unit cell dimensions are $221 \times 221 \mu \mathrm{m}$. The elastic moduli and thermal expansion coefficients of the phases are given in Table 4.1. The yield stress of the titanium matrix included in the table was used to ensure that the simulated response remained elastic, as plastic effects were not included in the CZM implementation in the present version. Given that the interfacial elastic parameters are not known for the SiC/Ti system, the value of the initial stiffness was chosen such that the initial response of the unit cell coincided with the experimentally measured transverse response of the unidirectional SiC/Ti composite. The value of the final separation distance was based on the maximum interfacial strength of 138 MPa (which coincides with the knee of the homogenized stress-strain curve observed experimentally) and the ratio between the elastic limit of initial separation and final separation of 10 suggested by Geubelle and Baylor (1998). Hence the only parameter that was varied, in order to determine the optimum value which produced the best correlation with experimental data 
during the interfacial degradation stage of the response, was interfacial strength. Table 4.2 summarizes these CZM parameters.

Table 4.2 Interfacial strength parameters for the SiC/Ti composite. Note: $k_{n}^{0}=\sigma_{\max } / \Delta_{n}^{o}$.

\begin{tabular}{|c|c|c|c|}
\hline$\sigma_{\max }=\tau_{\max }(\mathrm{MPa})$ & $k_{n}^{o}=k_{t}^{o}(\mathrm{MPa} / \mu \mathrm{m})$ & $\Delta_{n}^{o}=\Delta_{t}^{o}(\mu m)$ & $\Delta_{n}^{c}=\Delta_{t}^{c}(\mu m)$ \\
\hline 138 & 3000 & 0.0460 & 0.46 \\
\hline 70 & 3000 & 0.0233 & 0.46 \\
\hline 50 & 3000 & 0.0167 & 0.46 \\
\hline
\end{tabular}

In order to model residual stresses after consolidation, the unidirectional SiC/Ti composite was first subjected to a temperature cooldown which was followed by pure transverse loading. Following Johnson et al. (1990), temperature cooldown of $\Delta \mathrm{T}=-555^{\circ} \mathrm{C}$ was employed to simulate fabrication-induced residual stresses. This was based on the observation that any stresses that may develop during the fabrication process at the actual consolidation temperature greater than one half of the melting point of the matrix would be relieved by creep, Dieter (1976). The inclusion of residual stresses is critically important in correctly capturing the fiber/matrix interfacial separation mechanism owing to the high compressive radial stress at the interface that must be first overcome by the applied load. Two cases were then considered for comparison with the experimental data. First, the breaking of the chemical bond leading to fiber/matrix interfacial separation was modeled by the CZM during initial transverse loading immediately following fabrication cooldown in the absence of prior mechanical preload. Subsequently, the composite was subjected to transverse loading in the presence of residual stresses but with the chemical bond assumed to be destroyed over a certain arc length of the fiber/matrix interface by the initial preload. In this case, the interfacial separation occurred when the residual radial stress at the fiber/matrix interface was overcome by the applied transverse load. The individual results are presented in the sequel.

\subsection{Progressive Interfacial Degradation after Fabrication Cooldown}

The response of the SiC/Ti composite under transverse loading is simulated immediately after the fabrication cooldown when the fiber/matrix interface is presumed intact. In this case, the interface is assumed to be capable of supporting load in both normal and tangential directions. To demonstrate the importance of residual stresses, Figure 4.2 presents comparison of 
homogenized transverse responses generated using temperature changes of $\Delta \mathrm{T}=0^{\circ} \mathrm{C}$ and $\Delta \mathrm{T}=$ $555^{\circ} \mathrm{C}$ for interfacial strengths ranging from $138 \mathrm{MPa}$ to $0 \mathrm{MPa}$. For this comparison, the uncoupled interfacial separation law was employed. The asymptotic responses which converge to the same limiting behavior regardless of the interfacial strength magnitude, which also depend on the cooldown temperature, provide additional validation of the implemented cohesive zone model in the nonlinear region when the interface undergoes degradation. The same asymptotic response is expected upon complete separation of the fiber/matrix interface along the largest possible arc length before its arrest regardless of the interfacial strength magnitude, as was also observed by Raghavan and Ghosh (2005) in the absence of residual stresses. The presence of residual stresses shifts the asymptotic homogenized response upward as additional load is required to overcome the radial compressive stress at the fiber/matrix interface before separation initiates.

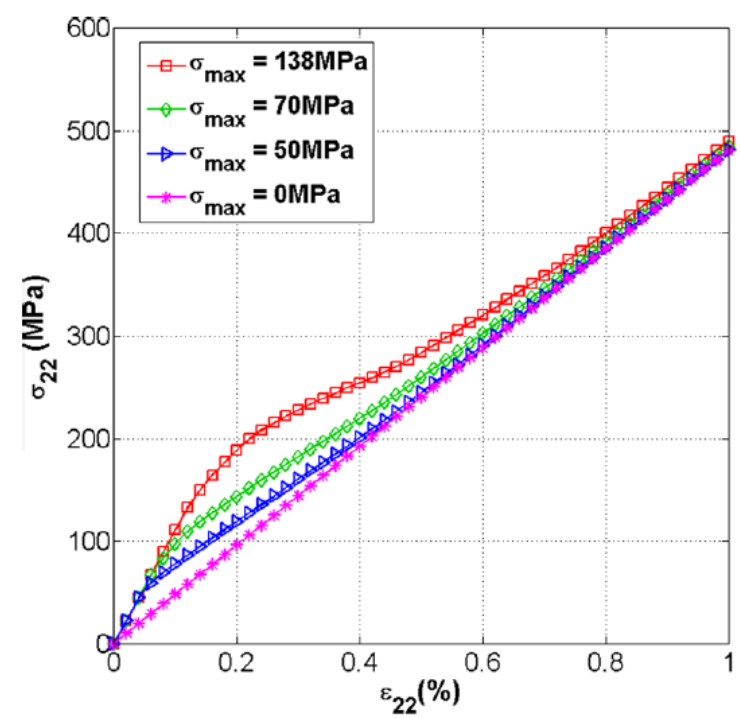

(a)

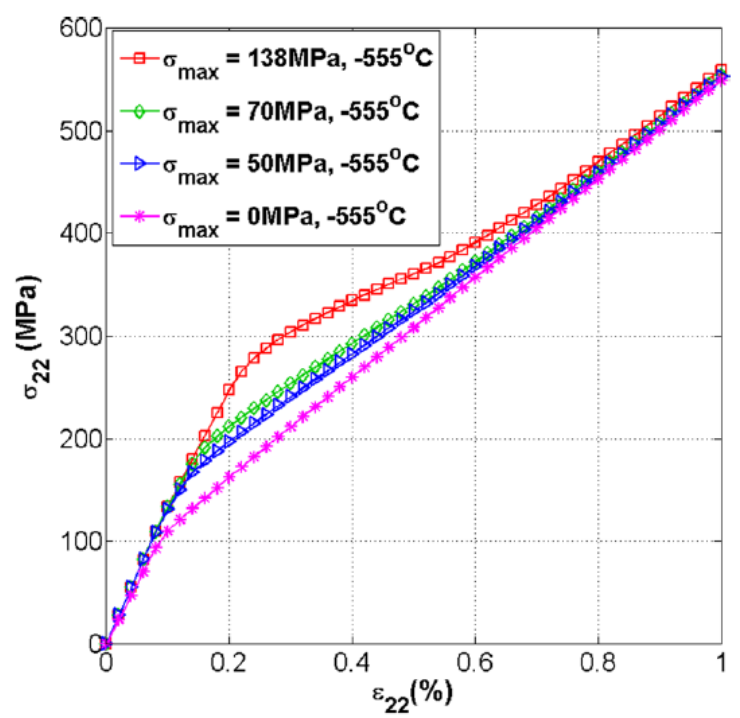

(b)

Figure 4.2 Initial transverse response of the unidirectional SiC/Ti composite with different interfacial strengths, illustrating the effect of fabrication-induced residual stresses. 

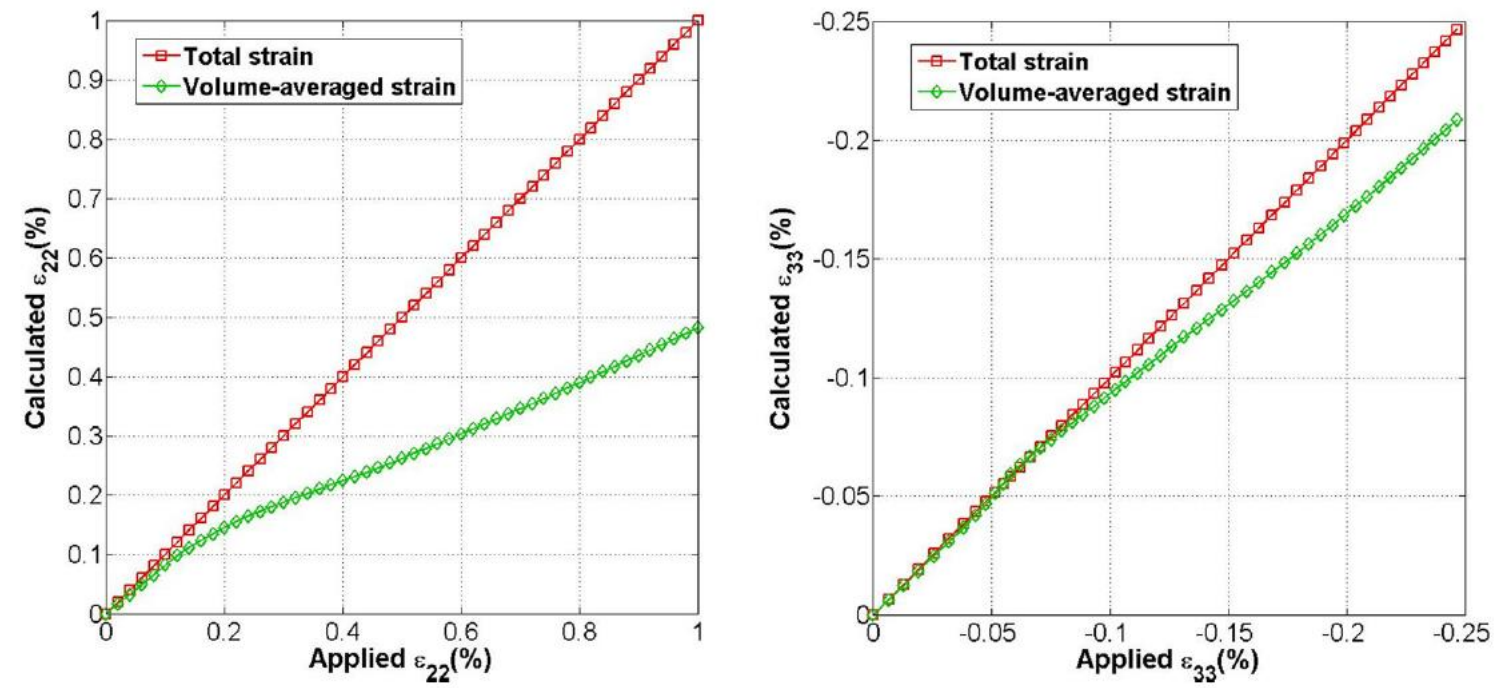

Figure 4.3 Comparison of the applied (vertical axis) and calculated (horizontal axis) homogenized strains based on Eq. (2.33), demonstrating consistency and accuracy of the implemented solution technique for the nonlinear response of the unit cell based on the implemented CZM, and importance of the contributions of the interfacial displacement discontinuities toward total homogenized strains.

Figure 4.3 provides a consistency and an accuracy check of the unit cell solution procedure in the presence of evolving damage through comparison of the applied homogenized strains that appear as loading parameters in the solution of the unit cell problem, Eqs. (2.182.21), and the corresponding homogenized strains calculated from the volume-averaged subvolume local strains and surface-averaged displacement discontinuities, Eq. (2.33). Included in the figure is the comparison between the applied homogenized strains and the volumeaveraged strains alone in order to demonstrate the contribution of the surface-averaged displacement discontinuities. The above results, generated using $\Delta \mathrm{T}=0^{\circ} \mathrm{C}$ and the interfacial strength of $138 \mathrm{MPa}$, provide additional confidence in the simulations that follow.

Comparison of the simulated results after the $\Delta \mathrm{T}=-555^{\circ} \mathrm{C}$ cooldown shown in Figure $4.2 \mathrm{~b}$ with experimental data of Johnson et al. (1990) reveals that the best correlation is obtained with the interfacial strength of $50 \mathrm{MPa}$. This comparison is illustrated in Figure 4.4 which also includes the corresponding comparison based on the coupled separation law with the interfacial strengths of $50 \mathrm{MPa}$ and $70 \mathrm{MPa}$. As anticipated from the graphical illustration of the interfacial separation laws shown in Figure 2.3, the use of the coupled separation law produces a softer response for the same interfacial strength of $50 \mathrm{MPa}$, requiring a higher interfacial strength to attain comparable accuracy, namely $70 \mathrm{MPa}$ in the present case. In subsequent presentation of the simulation results, we will employ the uncoupled separation law as it appears to yield 
somewhat better results. This difference, however, may be due to a number of factors which are beyond the scope of this work, including deviation from periodicity in the actual composite leading to unsymmmetric fiber/matrix separation.

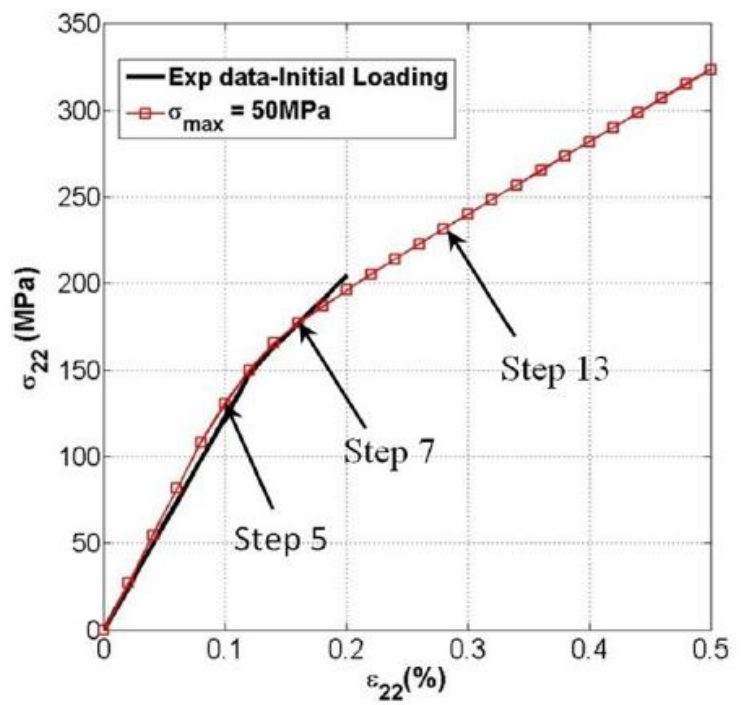

(a)

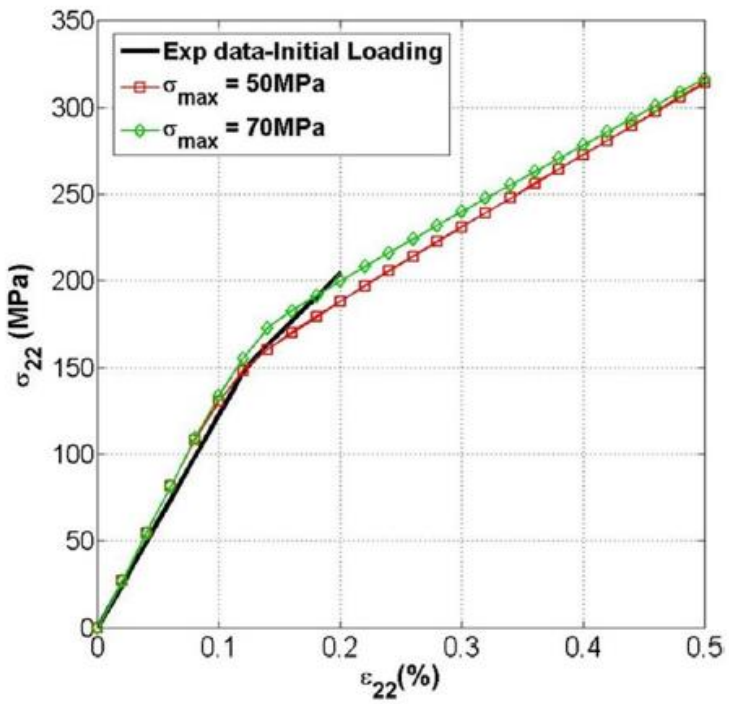

(b)

Figure 4.4 Initial transverse response of the unidirectional SiC/Ti composite immediately after fabrication cooldown, illustrating the effect of (a) uncoupled and (b) coupled interfacial separation laws.

Next, we examine the evolution of normal and tangential displacement discontinuities and tractions around the fiber/matrix interface and the corresponding stress field alterations at the different loading steps shown in Figure 4.4 for the uncoupled separation law. Figure 4.5 summarizes the distributions of normal and tangential displacement discontinuities and the corresponding tractions around the fiber/matrix interface, measured counterclockwise from the horizontal axis passing through the fiber's center, after the $555^{\circ} \mathrm{C}$ cooldown and at the subsequent mechanical loading steps 5, 7 and 13. During the cooldown cycle, neither interfacial normal opening nor tangential degradation occurs because the radial stress at the fiber/matrix interface is negative while the tangential stress does not exceed the interfacial shear strength. Hence the response remains linearly elastic. The normal interfacial tractions attain their maximum values at $\pm 45^{\circ}$ locations in the considered $\pm 90^{\circ}$ range due to the use of the square fiber array for the SiC/Ti composite. At these locations, the tangential tractions are zero while their maximum values occur at $-67.5^{\circ}$ and $+22.5^{\circ}$. During subsequent mechanical loading these locations are altered. 

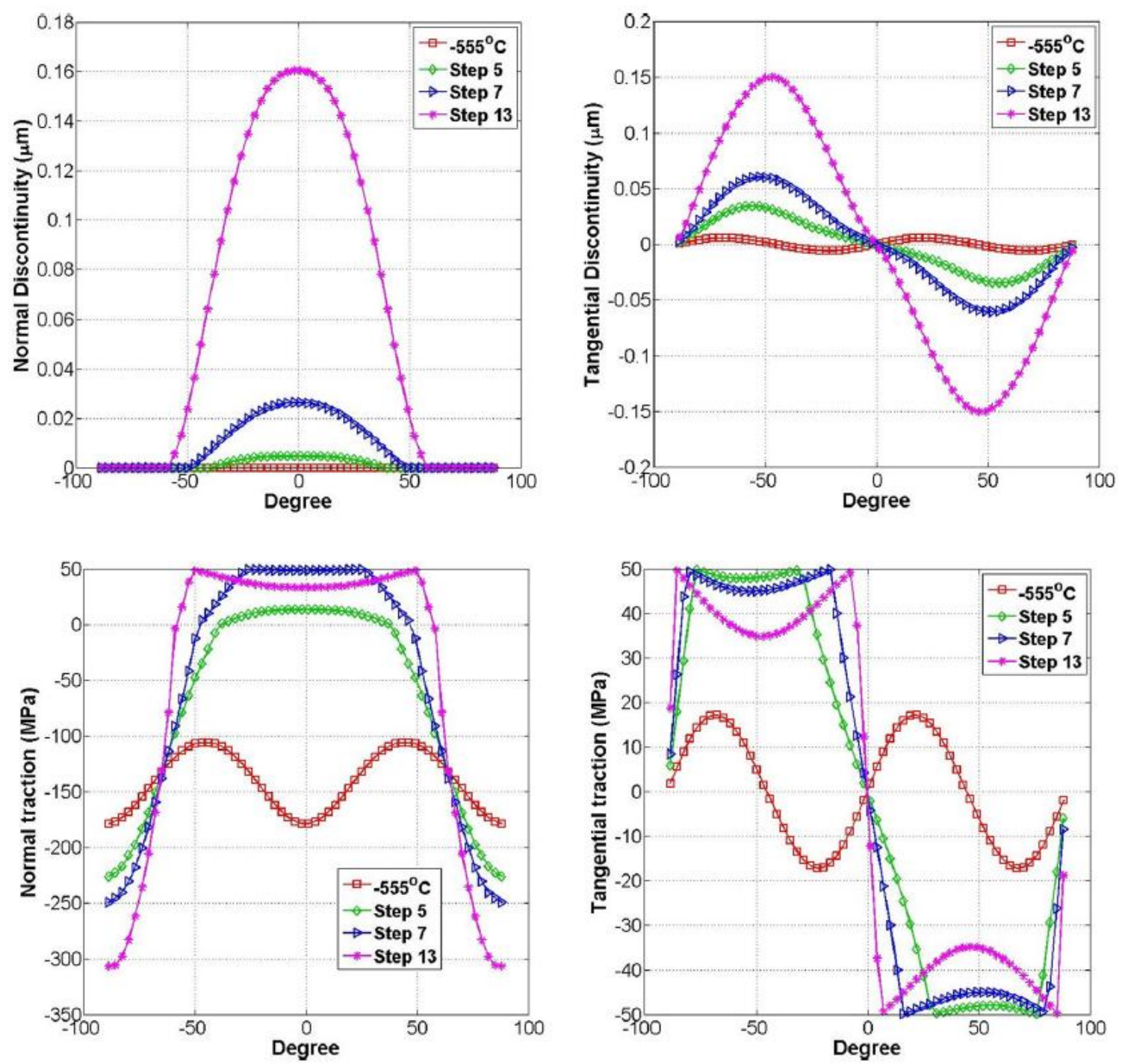

Figure 4.5 Interfacial displacement discontinuity and traction distributions around the fiber/matrix interface with progressively greater applied load after fabrication cooldown.

In order to initiate normal interfacial separation, the compressive residual radial stress must be overcome, which occurs between steps 4 and 5 at $0^{\circ}$ and is followed by interfacial elastic deformation until step 7 at which point the peak interfacial normal stress is attained along substantial portion of the interface spanning approximately $\pm 25^{\circ}$. Increasing loading produces increasing interfacial degradation accompanied by increasing normal separation characterized by increasing arc length and decreasing normal traction seen at step 13. The process is somewhat different for interfacial separation in the tangential direction. The interfacial sliding initiates much earlier (almost immediately after the cooldown), so that the interfacial shear strength is attained at step 4 (not shown). Hence at step 5 the interfacial shear separation is substantially 
larger in magnitude than the corresponding normal separation. These differences tend to disappear with increasing load as seen at step 13. Similar behavior is observed in the traction distributions after the interface degrades. The important phenomenon that occurs with increasing loading, which may be deduced from the above results, is the eventual arrest of the separation zone progression due to rapidly decreasing normal and tangential tractions outside of the separated zones around the fiber/matrix interface. This occurs at approximately $\pm 55^{\circ}$ and $\pm\left[85^{\circ} / 10^{\circ}\right]$ for the normal and tangential displacement discontinuities, respectively. In fact, the normal traction becomes compressive outside of the $\pm 55^{\circ}$ interval while the shear traction tends to zero outside of the $\pm\left[85^{\circ} / 10^{\circ}\right]$ intervals.

This section is concluded by presenting the full-field stress distributions in Figure 4.6 at the loading steps shown in Figure 4.4, including the three in-plane stresses $\sigma_{22}, \sigma_{33}, \sigma_{23}$ and the effective stress $\sigma_{e f f}$ which provides indication of yielding. These stress distributions demonstrate that the stress transfer mechanism from the region of the damage interface to the surrounding matrix is correctly captured by the implemented CZM into the FVDAM framework with fidelity comparable to an elasticity solution. In particular, as the interface loses its loadbearing capability, the matrix picks up a disproportionate portion of the applied horizontal load. We note that the effective yield stress at step 13 locally exceeds the yield stress of this particular titanium alloy in a small region at the apex of the fiber, requiring consideration of plasticity effects beyond this load step. 


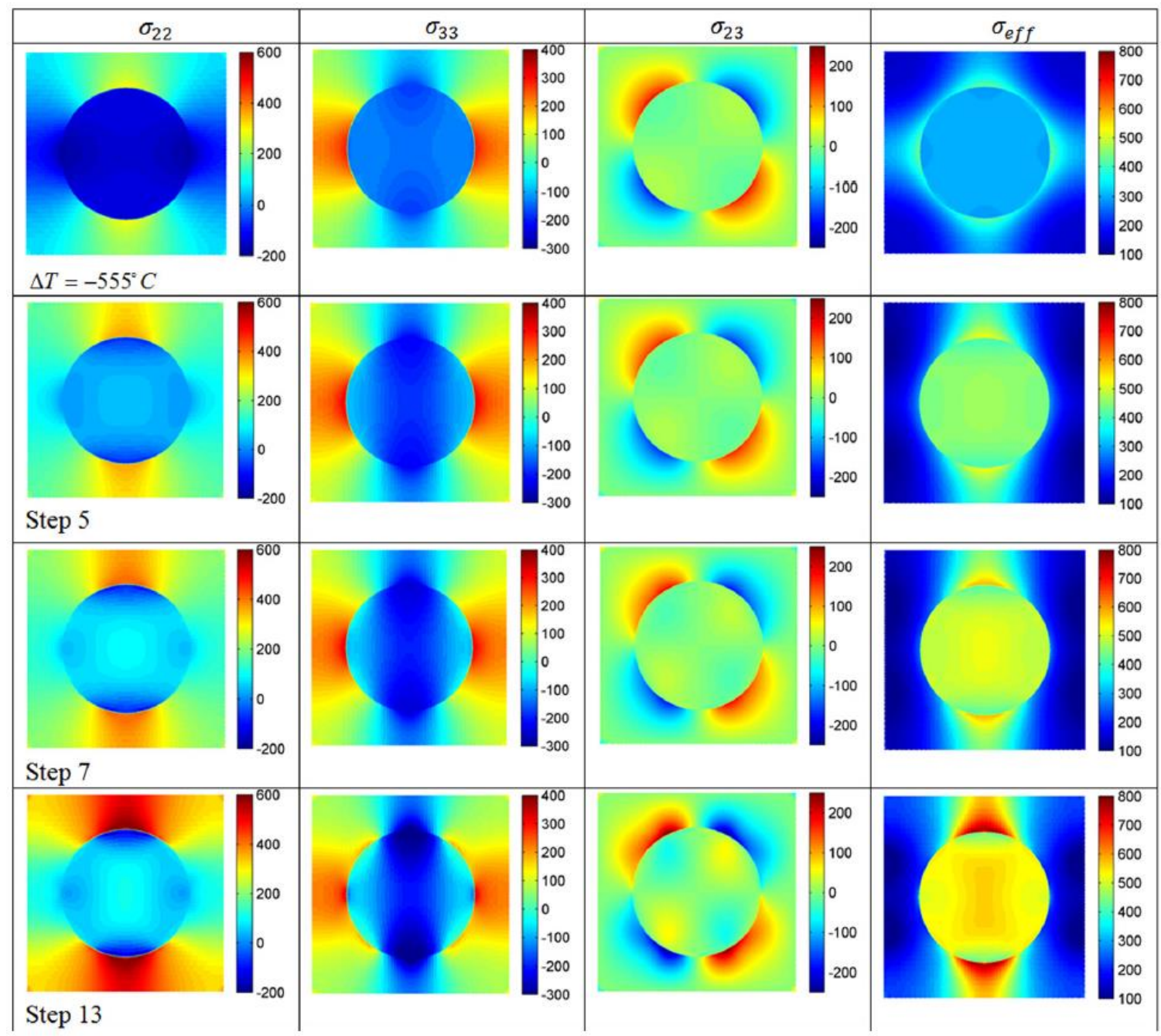

Figure 4.6 Full-field stress distributions at progressively greater applied load after fabrication cooldown.

\subsection{Progressive Interfacial Degradation after Initial Preload Cycles}

Initial loading cycles performed by Johnson et al. (1990) on the unidirectional SiC/Ti composite produced observable fiber/matrix separation of a certain length around the interface, effectively resulting in destruction of the chemical fiber/matrix bond in that region. The extent of this damage, that is the arc length of the interface that was damaged and the corresponding applied load, however, were not reported. Simulation of the subsequent response after initial pre-load cycles requires knowledge of the extent of this damage which has a substantial effect on the homogenized response. This is illustrated in Figure 4.7 for different lengths of damaged interface relative to the interface that is completely damaged around the entire circumference of the fiber. 
In performing these simulations, the traction-free condition in the damaged region was employed in the auxiliary system of equations used to solve the unit cell problem, while outside this region the interfacial separation law was applied. In the present case, the best correlation with experiment was obtained when the damage to the fiber/matrix interface caused by cyclic loading spans $\pm 45^{\circ}$ relative to the horizontal axis as also observed in Figure 4.7. This length is consistent with the results of Figure 4.5 which suggest that the maximum length of the fully degraded interface cannot exceed $\pm 55^{\circ}$. In fact, it is likely less than $\pm 50^{\circ}$ given that the maximum normal traction that the interface can support at the onset of degradation does not extend beyond this region.
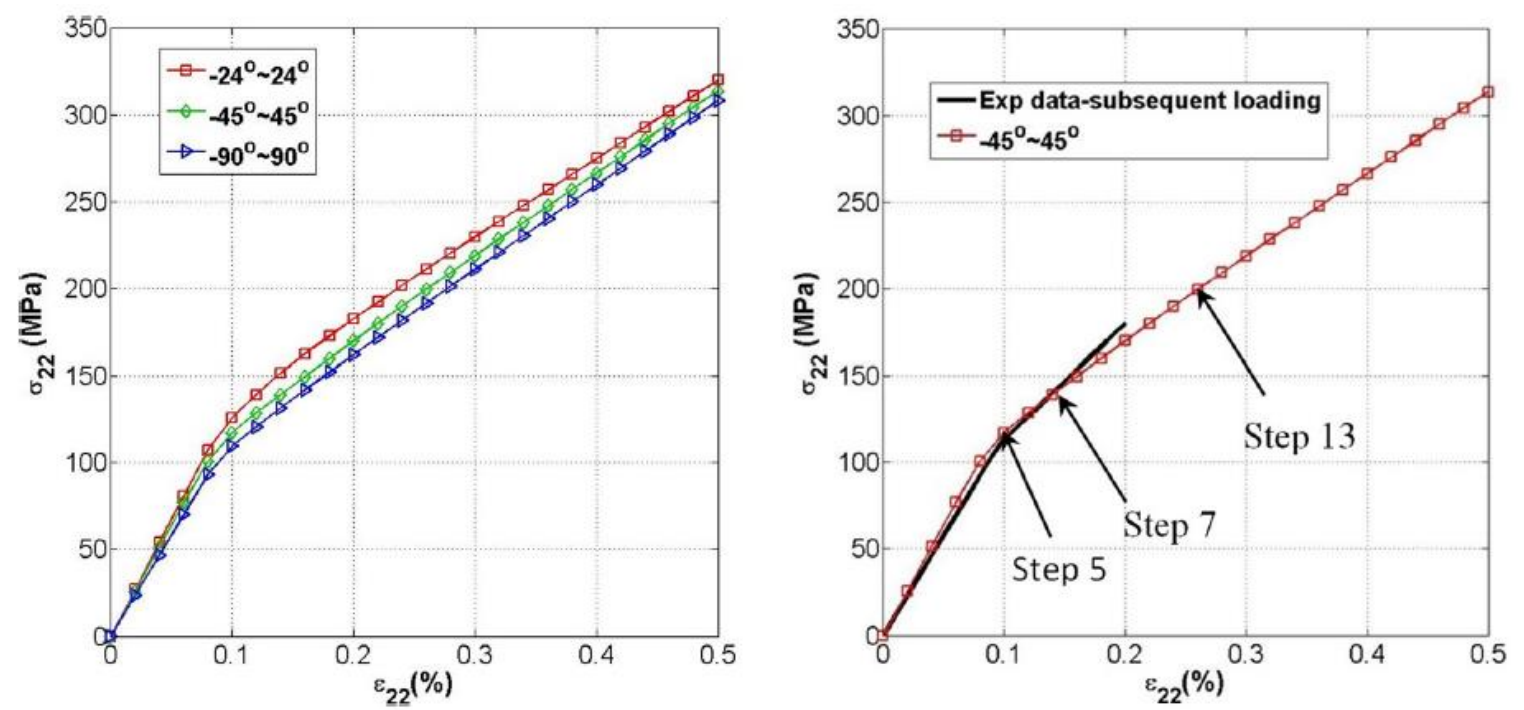

Figure 4.7 Transverse response of the representative unit cell of unidirectional SiC/Ti composite with different interfacial debonding lengths after initial preloading.

Figure 4.8 illustrates the distributions of normal and tangential displacement discontinuities and corresponding tractions around the fiber/matrix interface at the same subsequent loading steps used in the preceding section in the presence of intact interface after fabrication cooldown, namely 5, 7 and 13 which are also indicated in Figure 4.7. At step 5 the compressive radial stress is already overcome in the damaged region and the matrix separates from the fiber along the entire $\pm 45^{\circ}$ arc segment of the interface. The normal traction becomes zero in the separated region and negative outside. With the increase in loading, the magnitude of the normal displacement discontinuity increases and the fiber/matrix interfacial segment undergoing separation progresses further along the fiber's circumference. As expected, the displacement discontinuities at each load step are greater in this case relative to the initially 
intact interface simulations of the preceding section, Figure 4.5, due to the absence of normal traction which constrains the interfacial separation. The interfacial zone progression is accompanied by an increase in the normal traction outside of the zero-traction region that drives further separation and degradation as the normal traction reaches the interfacial strength. As in the preceding case, the interfacial separation process is arrested around $\pm 55^{\circ}$ as the normal traction decreases rapidly to a negative value outside of the separated zone. The tangential displacement discontinuity and traction distributions follow a similar pattern but the maximum values are attained much earlier as also observed in the preceding case.
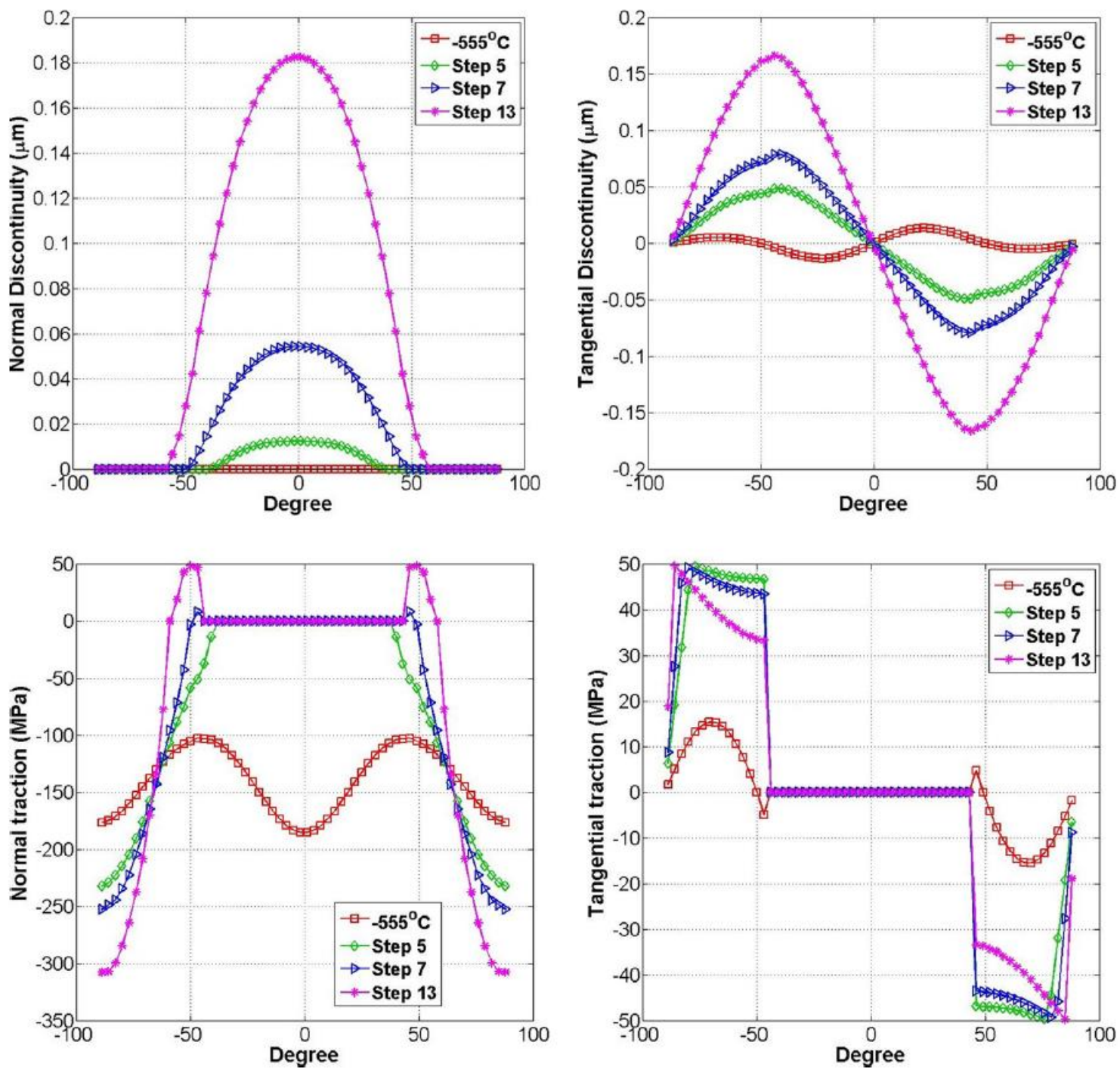

Figure 4.8 Interfacial displacement discontinuity and traction distributions around the fiber/matrix interface with progressively greater applied load after initial preloading. 


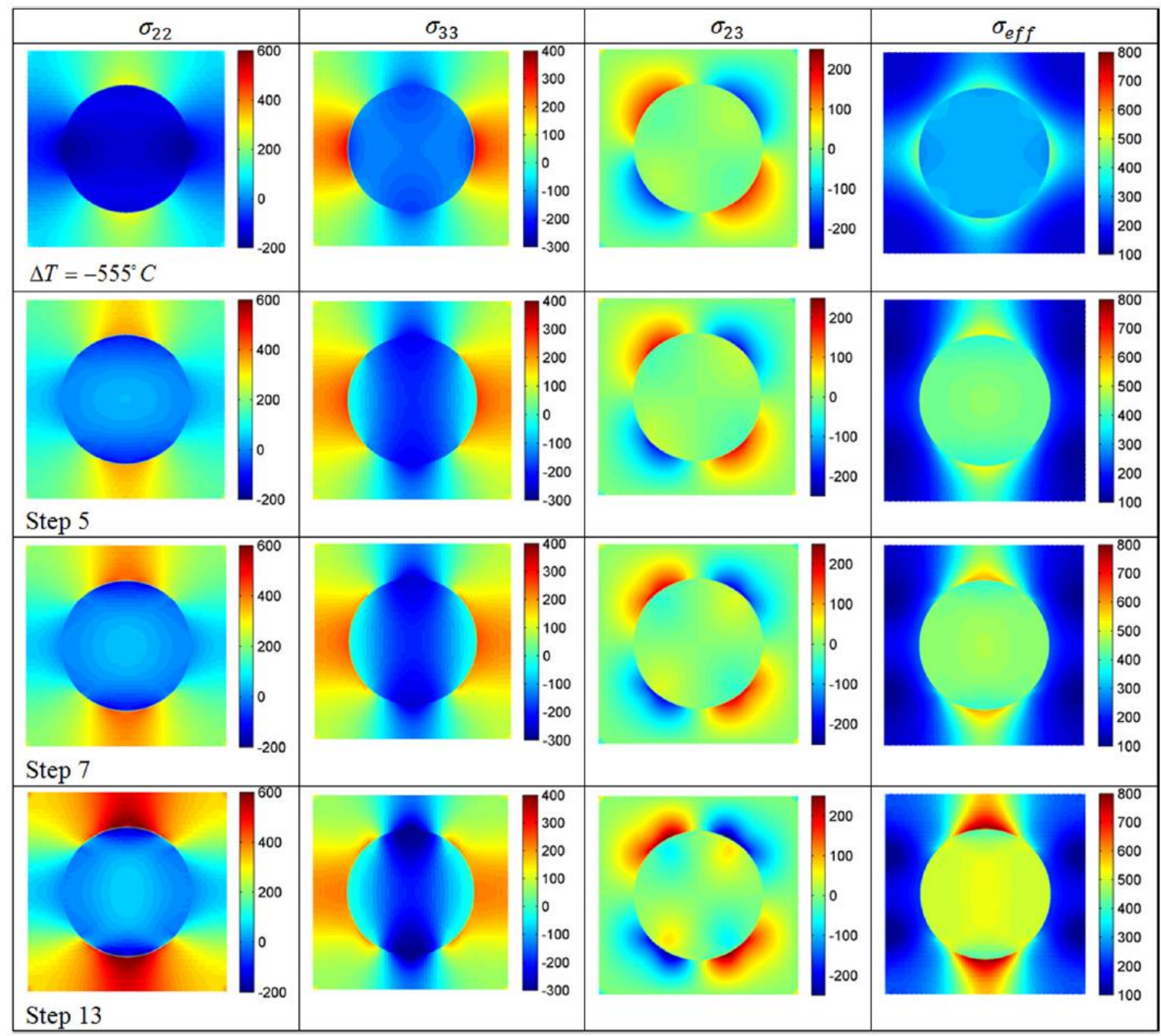

Figure 4.9 Full-field stress distributions at progressively greater applied load after fiber/matrix degradation by initial preloading.

Figure 4.9 illustrates the full-field distributions of the three in-plane stresses $\sigma_{22}, \sigma_{33}, \sigma_{23}$ and the effective stress $\sigma_{e f f}$ for comparison with those of Figure 4.6 with the initially intact interface. The rapid opening of the damaged interface once the compressive residual stresses are overcome renders the matrix material directly in front of the separated traction-free interface not as effective in supporting the applied horizontal load, producing lower magnitudes of the $\sigma_{22}$ stress component. This is accompanied by visibly lower magnitudes of the $\sigma_{22}$ stress component in this region due to the reduced constraint of the separated interface. 


\subsection{Effect of Unit Cell Architecture on Interfacial Damage}

Following the investigation of progressive interfacial degradation after fabrication cooldown with square unit cell, the hexagonal unit cell is utilized to generate homogenized response and investigate the interfacial degradation between fiber and matrix. Fiber and matrix properties in Table 4.1 are used and uncoupled bilinear cohesive law is adopted with interfacial strength of $50 \mathrm{MPa}$ (see other interfacial parameters in Table 4.2 for reference). This set of material properties generates the best correlation with the experimental response of $\mathrm{SiC} / \mathrm{Ti}$ composite based on the square unit cell under transverse loading after fabrication cool-down, and the corresponding homogenized response and interfacial quantities are employed to compare the results generated using the hexagonal unit cell.

Figure 4.10 shows the hexagonal unit cell geometry and discretization, as well as the fiber/matrix interfaces highlighted by red lines. The subvolume size in the hexagonal unit cell is similar to the size of subvolumes in the square unit cell shown in Figure 4.1, and the hexagonal unit cell with the size of $474.4 \mu \mathrm{m}$ in length direction and $410.8 \mu \mathrm{m}$ in width direction is discretized into 120x100 subvolumes. Figure 4.11 illustrates the comparison of homogenized responses generated using square and hexagonal unit cells, with both exhibiting good agreement with experimental data. Before step 13, both responses are very close, while after step 13 the response based on the hexagonal unit cell becomes more compliant.

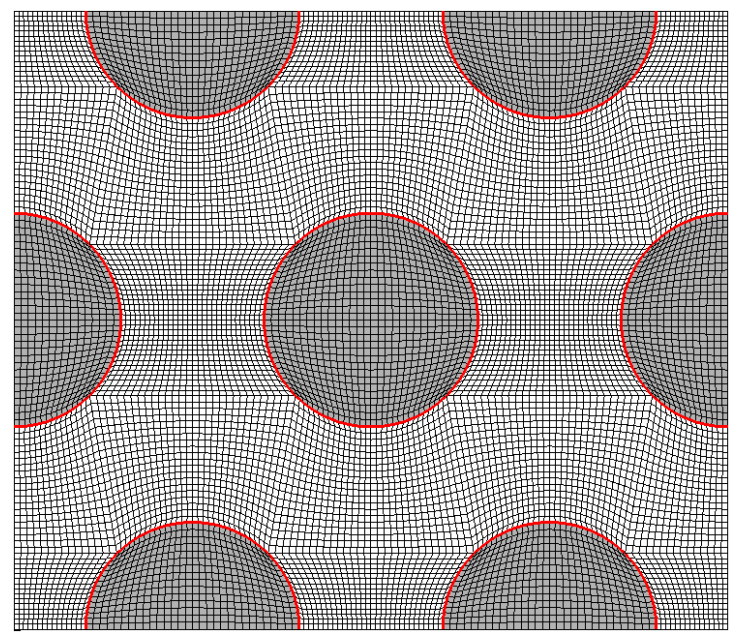

Figure 4.10 Unit cell geometry and discretization containing 0.325 fiber volume fraction. 


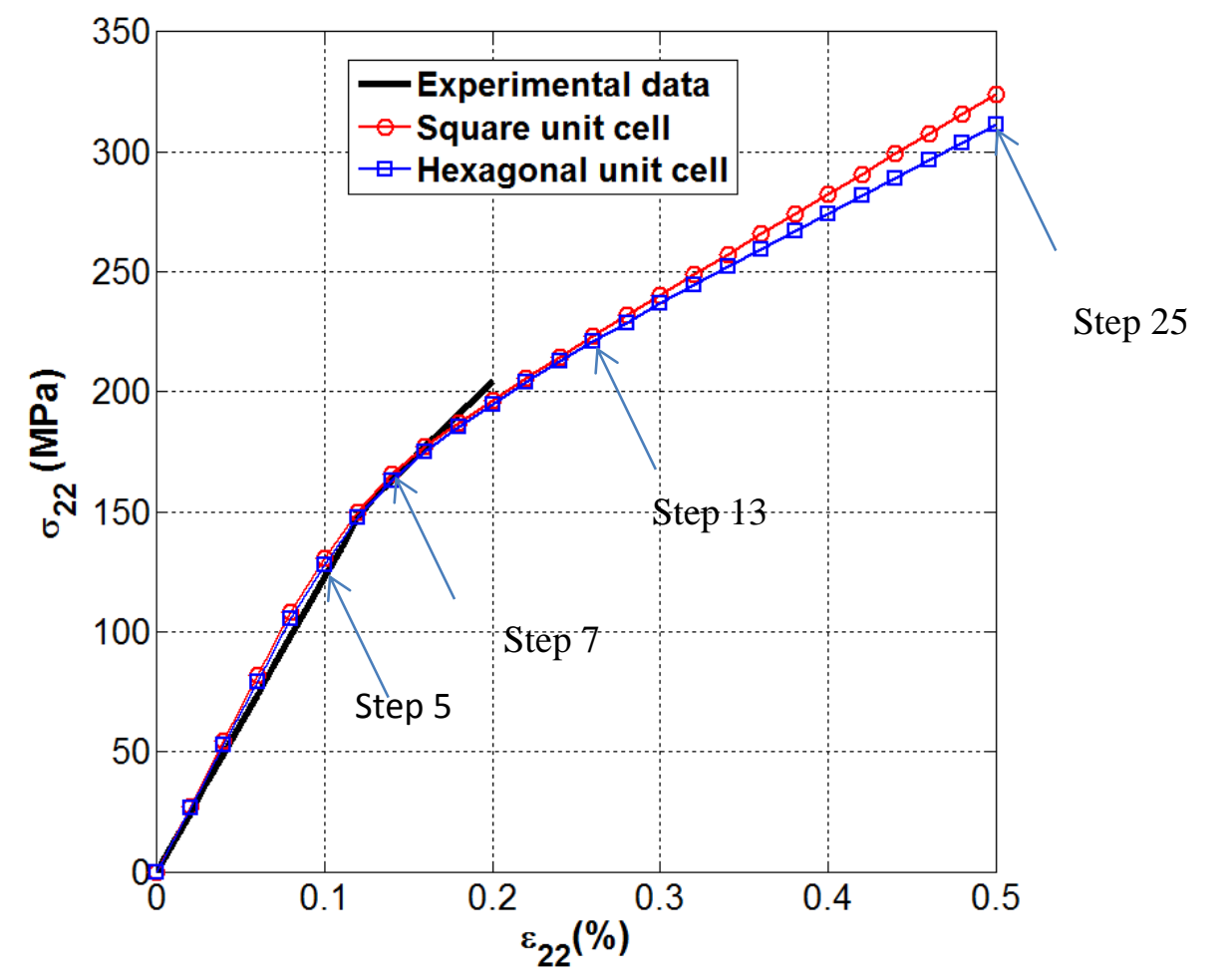

Figure 4.11 Initial transverse response generated via square and hexagonal unit cells for unidirectional $\mathrm{SiC} / \mathrm{Ti}$ composite immediately after fabrication cooldown with uncoupled separation laws.

Figure 4.12 illustrates the comparison of interfacial displacement discontinuity and traction distributions around the fiber/matrix interface after the $-555^{\circ} \mathrm{C}$ fabrication cool-down. As shown in this figure, normal compressive stress develops around the fiber/matrix interface because of mismatch of thermal expansion coefficients between fiber and matrix. Moreover, the normal displacement discontinuity is zero because when the interface is under compression, the corresponding displacement discontinuity is replaced by the displacement continuity condition. This is in contrast with the major finite-element packages such as Abaqus and ANSYS wherein high stiffness is used to resist interpenetration. Meanwhile it is easy to observe that normal traction, tangential displacement discontinuity and tangential traction distributions for the hexagonal unit cell are more uniform compared with the results from square unit cell, because the fiber in hexagonal unit cell is more uniformly distributed inside the matrix. 

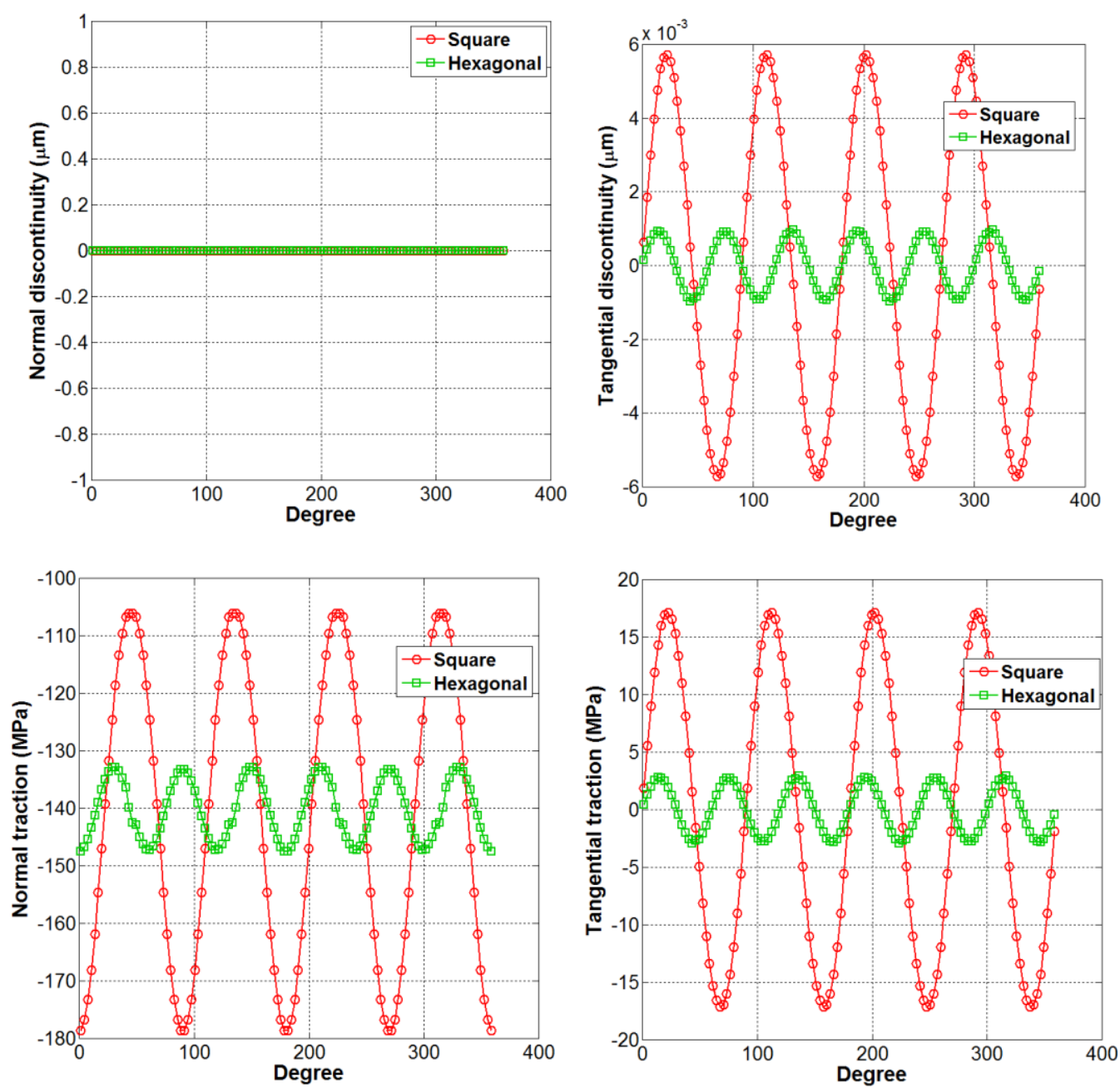

Figure 4.12 Interfacial displacement discontinuity and traction distributions around the fiber/matrix interface under $-555^{\circ} \mathrm{C}$ cool-down.

Figure 4.13 shows the interfacial displacement discontinuity and traction distributions around the fiber/matrix interface at step 5. In normal direction, interface in the area around $0^{0}$ and $180^{\circ}$ overcomes the compressive stress and maximal interfacial opening and stress appear in the hexagonal unit cell while larger interfacial area around the top and bottom of fiber is under high compressive stress. Moreover the compressive stress in square unit cell is almost 50\% higher than the stress in hexagonal unit cell. In tangential direction, interface starts experiencing degradation in contrast to the on-set elastic interfacial opening in normal direction and interface in square unit cell experiences more severe degradation than interface in hexagonal unit cell, while the position with most severe degradation in those two unit cells are nearby but not exactly the same. 

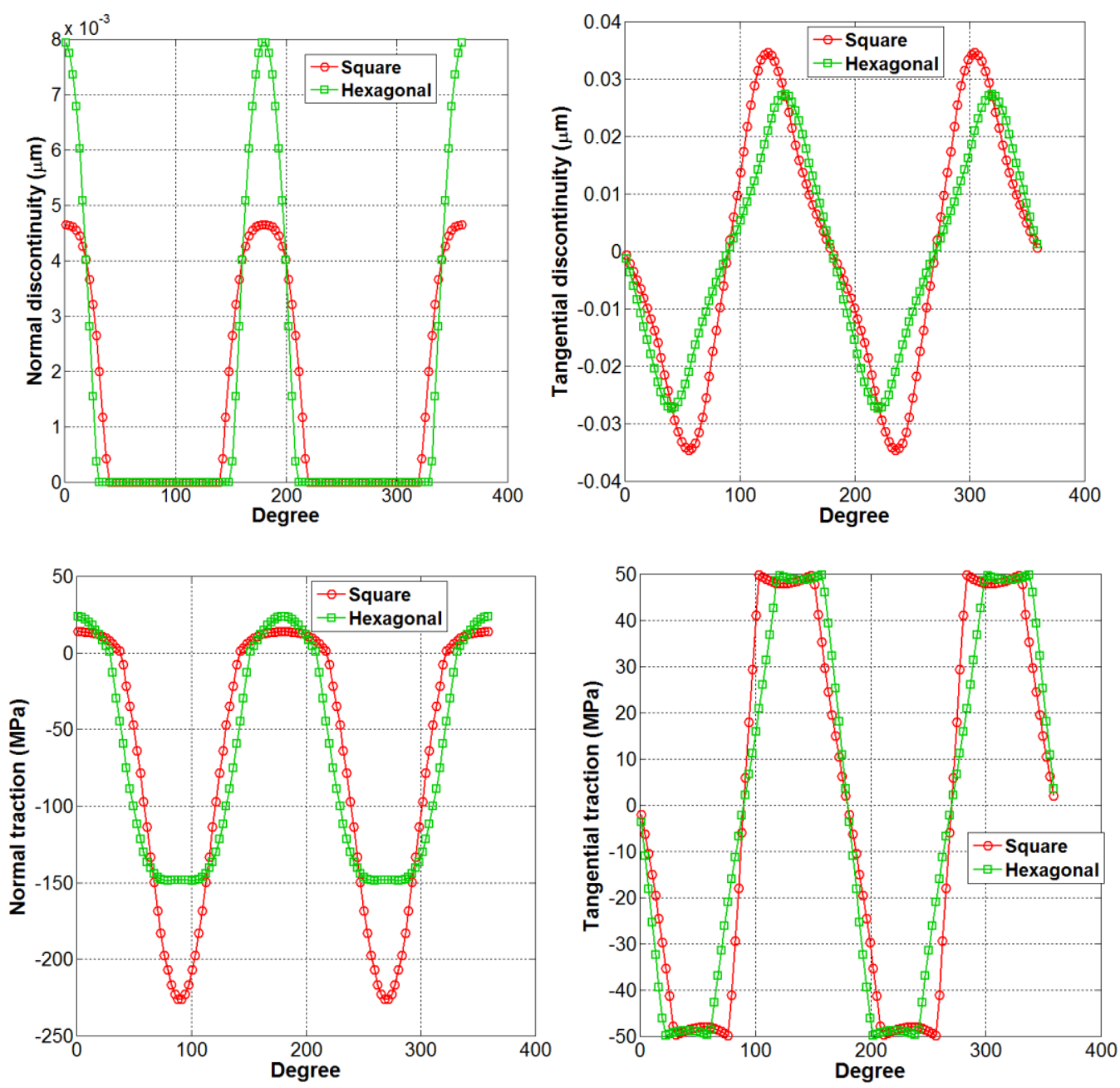

Figure 4.13 Interfacial displacement discontinuity and traction distributions around the fiber/matrix interface at step 5 .

Figure 4.14 shows the interfacial displacement discontinuity and traction distributions around the fiber/matrix interface at step 13. In normal direction, interface in hexagonal unit cell degrades more severely and in tangential direction interface has similar response. And these explain the slight compliant response at step 13. Moreover the difference of maximal compressive stress between square unit cell and hexagonal unit cell decreases to $25 \%$ at this step from 50\% at step 5. After step 13 the difference between homogenized responses of hexagonal unit cell and square unit cell will continue to deviate. As shown in figure 4.15 at step 25 distributions of interfacial qualities possess very similar shape except for that interface of hexagonal unit cell experiences more severe damage in both normal and tangential direction, which cause the more compliant response in the homogenized response. 

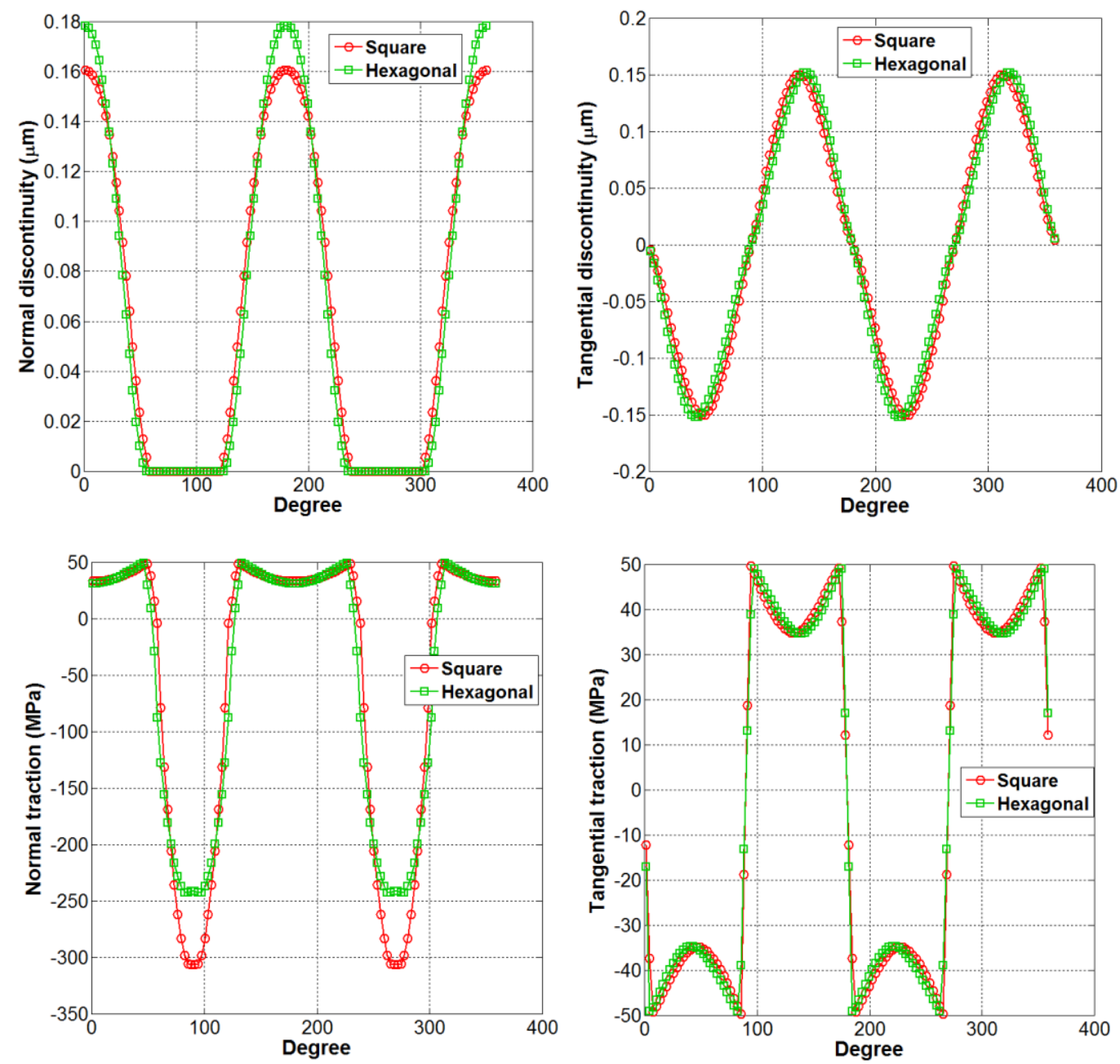

Figure 4.14 Interfacial displacement discontinuity and traction distributions around the fiber/matrix interface at step 13.

Figure 4.16 and Figure 4.17 show the full-field stress distributions of hexagonal and square unit cells after $555^{\circ} \mathrm{C}$ fabrication cool-down and at loading step 13, at which interface experiences certain degree of degradation as shown in Figure 4.14. First of all, after $555^{\circ} \mathrm{C} \mathrm{cool-}$ down, stress distributions of $\sigma_{22}$ and $\sigma_{33}$ in hexagonal unit cell are not equal to each other after 90 degrees' rotation in contrast to the stresses in square unit cell, because in hexagonal unit cell the fiber distribution pattern varies after 90 degrees' rotation. Second of all, in both normal stress components of $\sigma_{22}$ and $\sigma_{33}$, the compressive stress is higher in square unit cell and this causes higher effective stress, which is more uniformly distributed in hexagonal unit cell with hexagonal shape comparing with the more localized distribution in square unit cell with square shape. Moreover the shear stress $\sigma_{23}$ is higher in hexagonal unit cell comparing with the corresponding shear stress in square unit cell. 

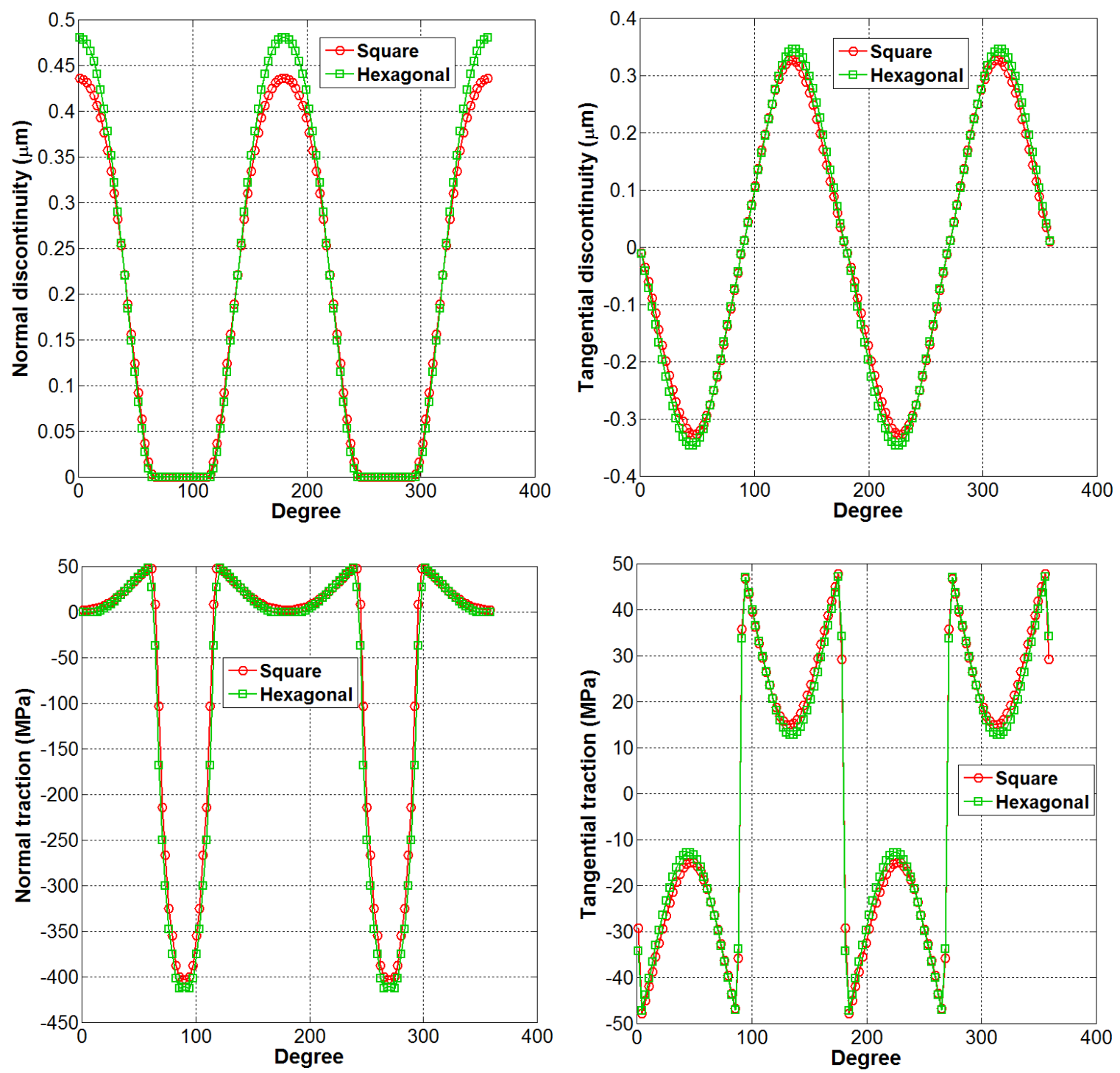

Figure 4.15 Interfacial displacement discontinuity and traction distributions around the fiber/matrix interface at step 25.

Figure 4.17 shows the full-filed stress distributions at step 13, at which interface in both normal and tangential direction experiences some degree of degradation as shown in Figure 4.14. As the interfacial degradation happens, which reduces stress transfer ability from fiber to matrix along left and right hand sides of interface, high tensile $\sigma_{22}$ and compressive $\sigma_{33}$ are developed around the top and bottom area of fiber, which cause the high effective stress in the matrix around fiber top and bottom area. Also interfacial degradation is more severe in square unit cell than the degradation in hexagonal unit cell and this cause more dramatically loading shifting effects in square unit cell, which has higher effective stress around the top and bottom fiber area. 
Moreover it's known from Figure 4.13 shear stress is higher in hexagonal unit cell than the stress in square unit cell after fabrication cool-down, while after interfacial degradation, the shear stress distribution are fairly close to each other, which also can be reflected from the tangential interfacial stress distribution in Figure 4.14.

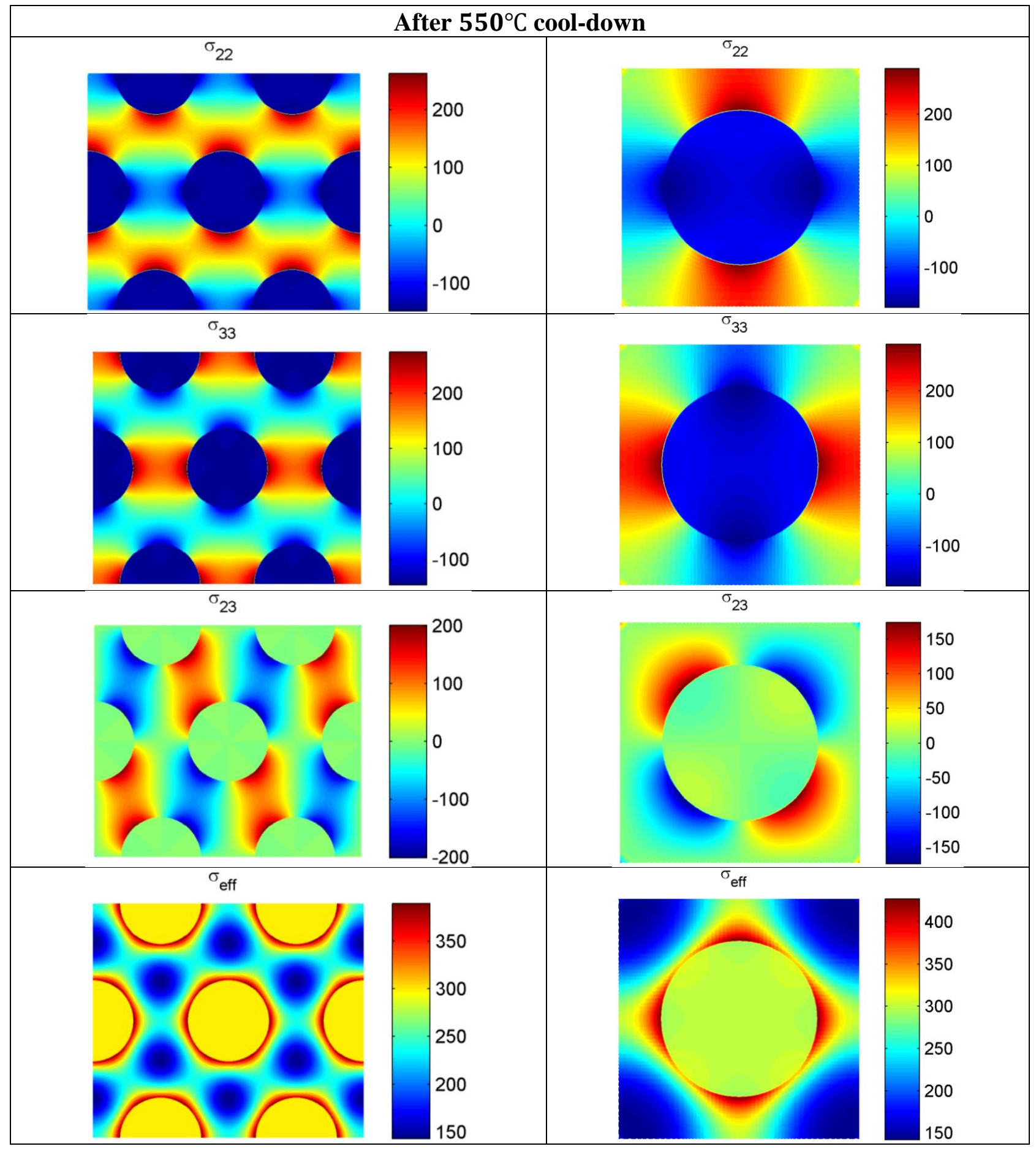

Figure 4.16 Full-field stress distributions after $555^{\circ} \mathrm{C}$ fabrication cool-down. 


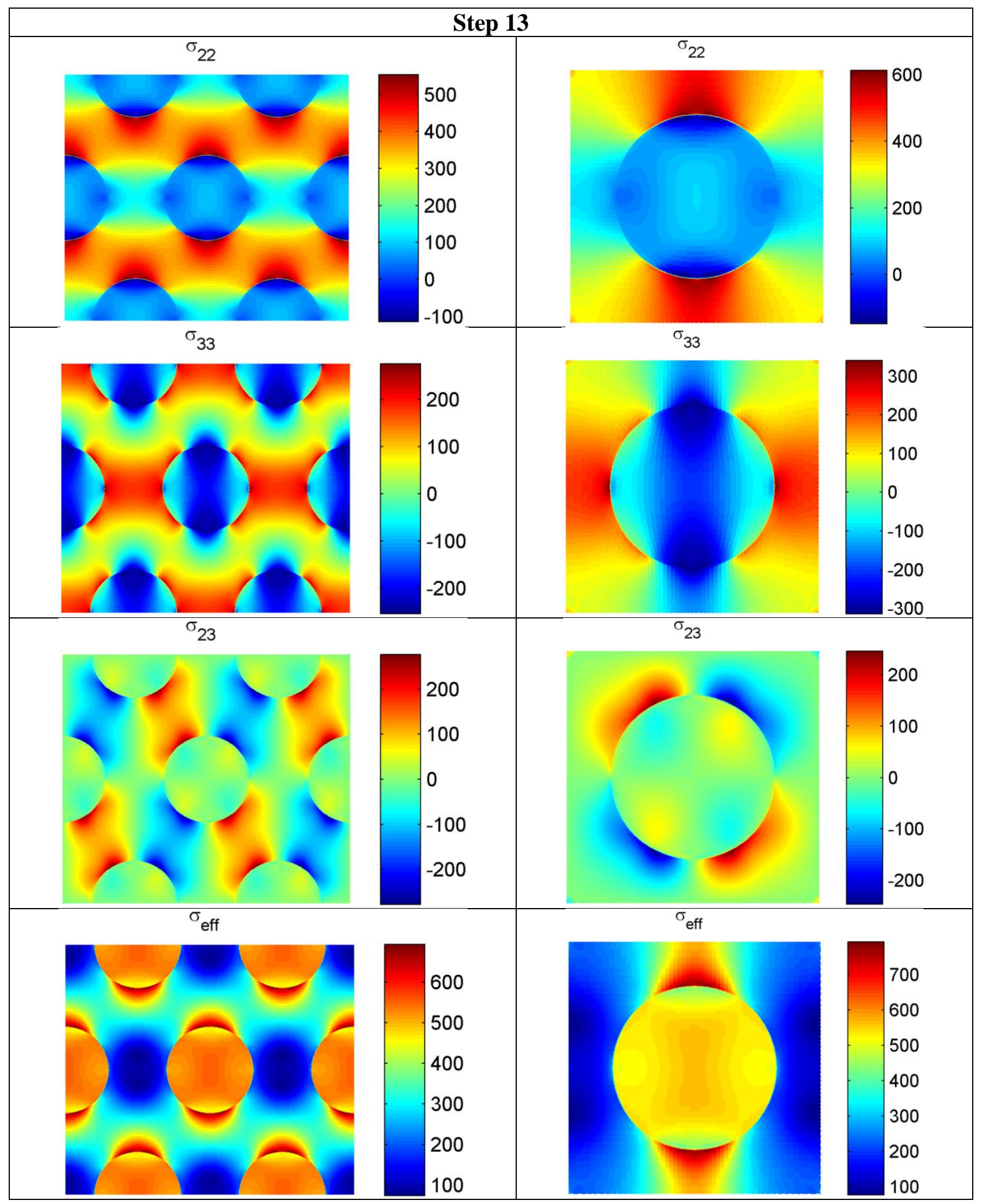

Figure 4.17 Full-field stress distributions at step 13. 


\subsection{Effect of Loading Direction for Hexagonal Unit Cell}

In this section, we look into the effect of loading directions on the homogenized response of the hexagonal unit cell and the associated fiber/matrix interfacial behavior. Two types of loading conditions are applied to the unit cell: one is transverse loading, denoted by 22 direction in Figure 4.18, which was used in Section 4.4 for comparison with the square unit cell response, and another one is through-thickness loading direction, denoted by 33 direction in Figure 4.18. Figure 4.18 illustrates the homogenized responses of square and hexagonal unit cells under transverse and through-thickness loadings for unidirectional SiC/Ti composite with uncoupled separation laws immediately after fabrication cooldown. As expected, the response of the square unit cell under those two loadings is exactly the same because the interface profile corresponding to either of the two loading directions does not change upon 90 degree rotation. For the hexagonal unit cell in the elastic stage the homogenized response is the same because of the transversely isotropic relation $E_{22}=E_{33}$. The onset of interfacial degradation leads to anisotropic behavior, with the homogenized response along 33 direction more compliant than the response along 22 direction.

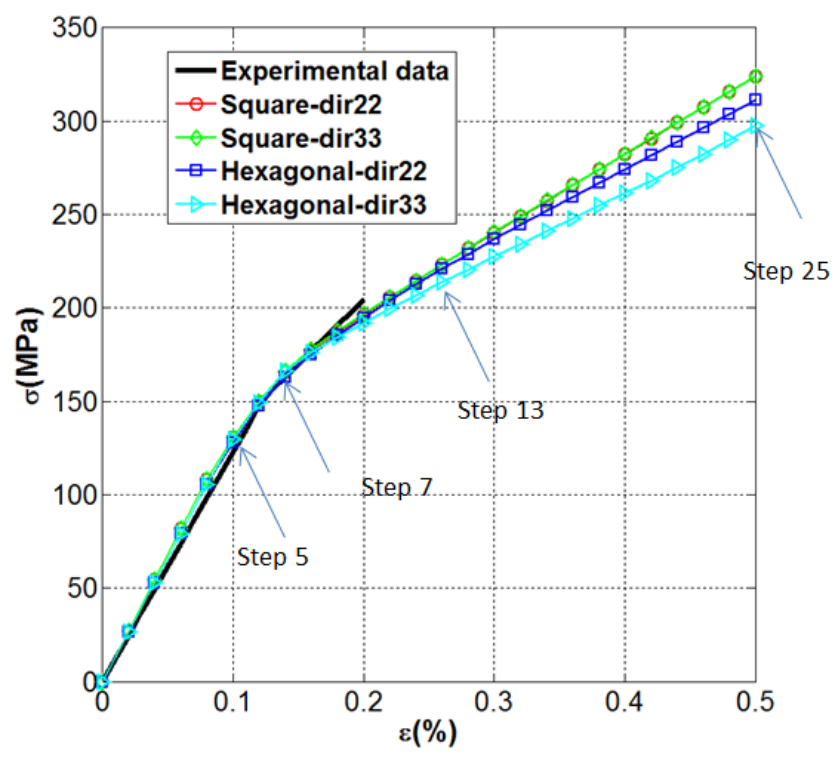

Figure 4.18 Initial transverse and through-thickness response generated via square and hexagonal unit cells for unidirectional $\mathrm{SiC} / \mathrm{Ti}$ composite immediately after fabrication cooldown with uncoupled separation laws.

Figure 4.19 and 4.20 illustrate the interfacial displacement discontinuity and traction distributions around the central fiber/matrix interface at steps 5 and 13 for the hexagonal unit cell 
subjected to transverse and through-thickness loading. Since the two loading directions differ by 90 degrees, the corresponding maximum interfacial openings also differ by 90 degrees. For comparison purposes, interfacial quantity distributions under transverse loading are plotted from the outer right point along the counterclockwise direction, and the interfacial quantity distributions under through-thickness loading are plotted from the top point along counterclockwise direction. At step 5, interfacial deformation in the normal direction is in the elastic stage and interface opening under transverse loading is slightly higher than under through-thickness loading. In the tangential direction, the interface under both loading types degrades slightly and the interface under transverse loading experiences slightly more severe degradation. Because the interfacial opening difference is small and interfacial damage in the tangential direction is in its early stage, the loss of loading capability for both unit cells is negligible and therefore the homogenized properties are nearly the same. At step 13 the interface under both loading types experiences moderate interfacial damage and the homogenized response of the unit cell under through-thickness loading is more compliant than the response under transverse loading. As shown in Figure 4.20, the interface in the normal direction under through-thickness loading experiences more severe damage than under transverse loading. In contrast, in the tangential direction the interface under both loading types experiences similar damage. Because of more severe interfacial damage in the normal direction under throughthickness loading, which causes more severe loss of load transfer ability, the homogenized response is more compliant than the response under transverse loading.

Figures 4.21 and 4.22 illustrate the full-field stress distributions in the hexagonal unit cell subjected to transverse and through-thickness loadings at steps 5 and 13. Because of the 90 degree difference between loading directions, we should compare stress $\sigma_{22}$ with $\sigma_{33}$ and stress $\sigma_{33}$ with $\sigma_{22}$. In Figure 4.21 the maximum and minimum values of the normal stress $\sigma_{22}$ along the transverse loading direction is similar to the value of the normal stress $\sigma_{33}$ along the throughthickness direction. The shear stress $\sigma_{23}$ distribution patterns for both loading types are also similar while the shear stress magnitude is higher under transverse loading. Regarding effective stress, the maximum value is higher under through-thickness loading because of the higher compressive normal stress along the left and right sides of the fiber illustrated in Figure 4.20. With the development of interfacial damage, the maximum and minimum values of the normal stress $\sigma_{22}$ along the transverse loading direction are similar to the values of the normal stress 
$\sigma_{33}$ along the through-thickness direction, Figure 4.22. The shear stress $\sigma_{23}$ distribution patterns as well as the magnitudes are similar. Regarding the effective stress, high values are more localized in the top and bottom areas of the fiber under transverse loading, and to the left and right of the fiber under through-thickness loading. This occurs because the normal interfacial damage and maximum stress are slightly higher under the through-thickness loading due to the relatively high compressive normal stress, as seen in Figure 4.20.
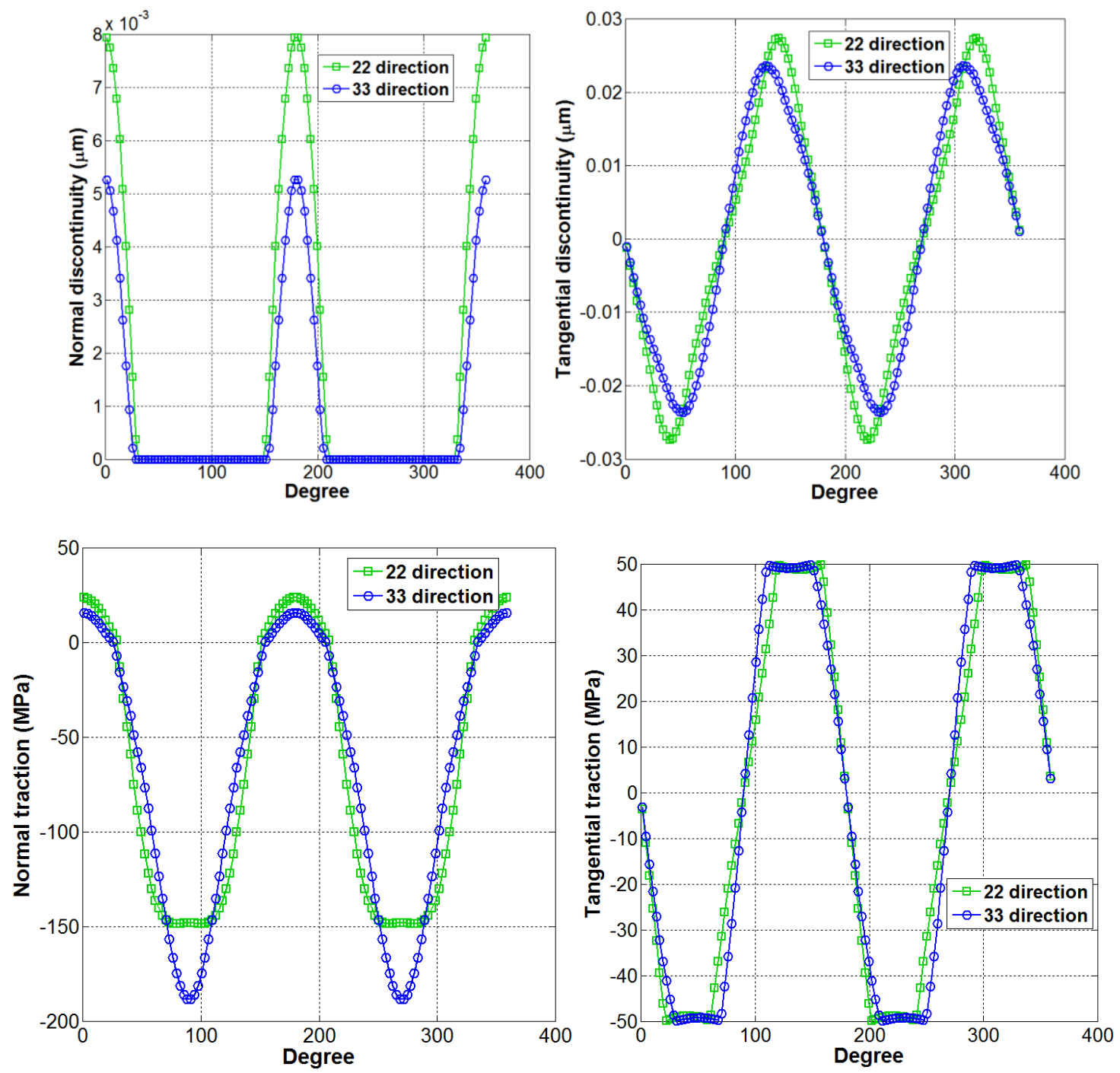

Figure 4.19 Interfacial displacement discontinuity and traction distributions around the fiber/matrix interface at step 5 under transverse and through-thickness loadings. 

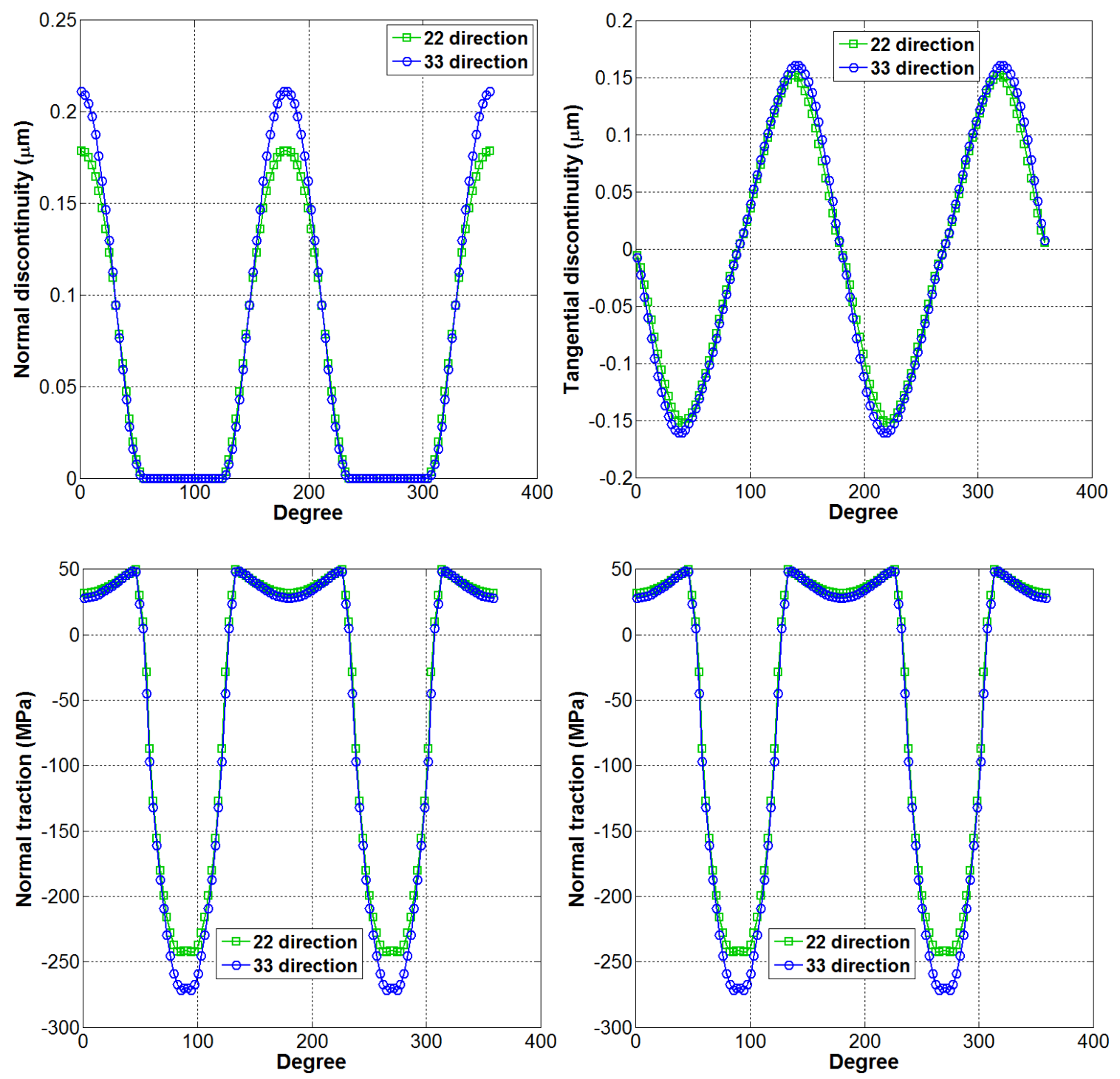

Figure 4.20 Interfacial displacement discontinuity and traction distributions around the fiber/matrix interface at step 13 under transverse and through-thickness loading. 


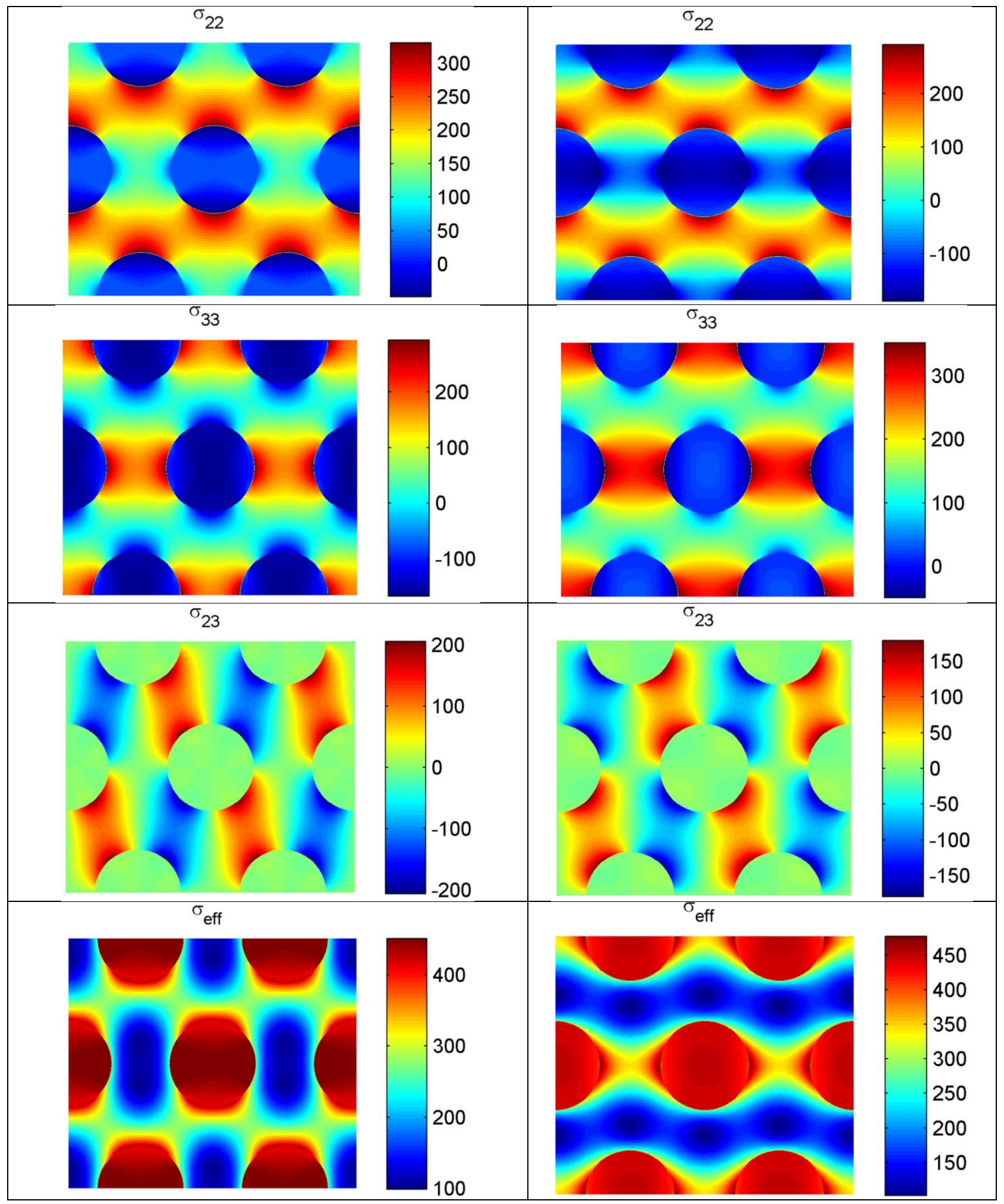

Figure 4.21 Full-field stress distributions at step 5 along transverse loading (left) and along through-thickness loading (right). 


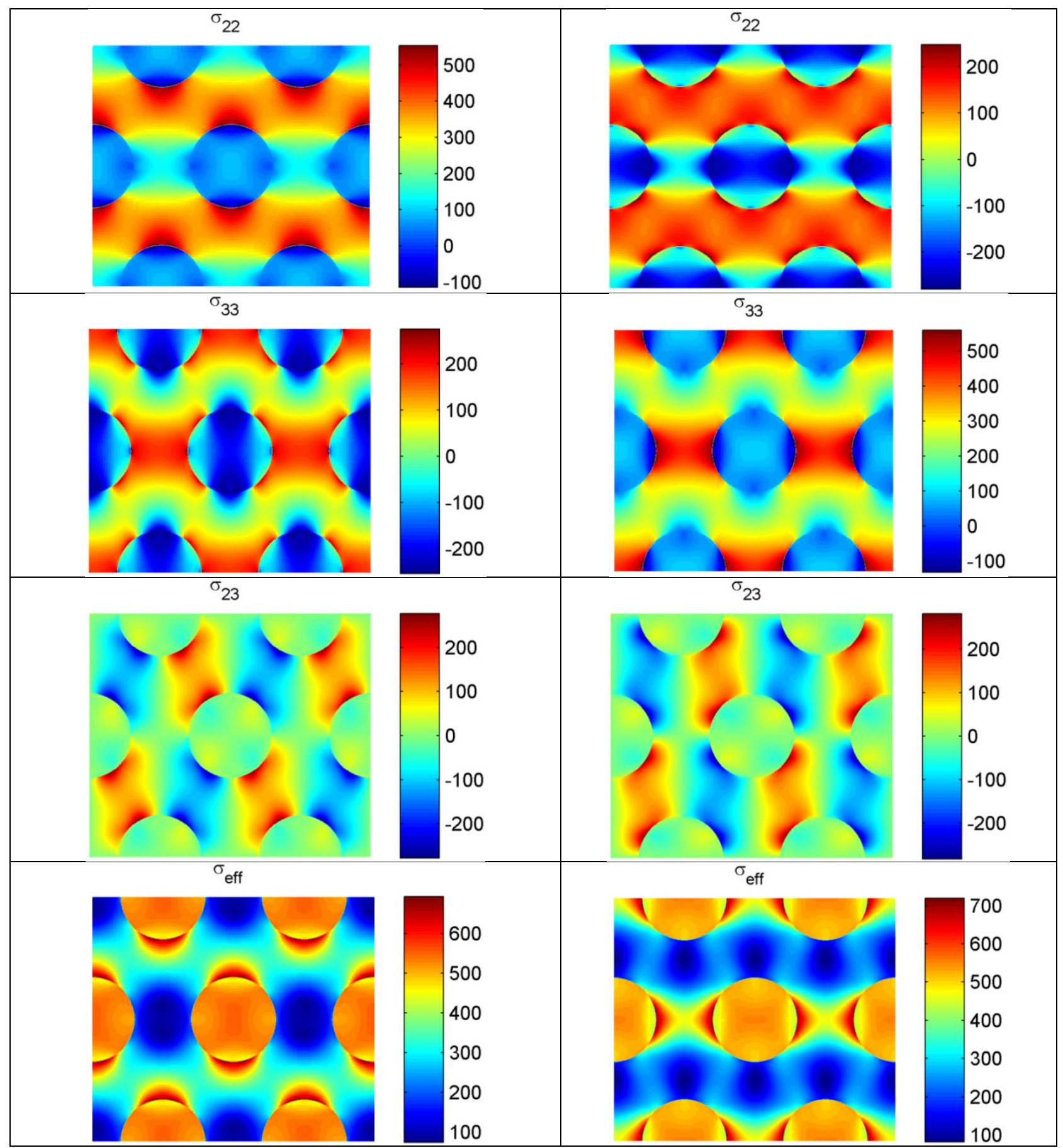

Figure 4.22 Full-field stress distributions at step 13 along transverse loading (left) and along through-thickness loading (right).

\subsection{Discussion}

The generated results demonstrate that the 0th-order version of the parametric FVDAM theory is capable of accurately capturing interfacial displacement discontinuity and corresponding traction 
distributions along interfaces undergoing separation based on the bilinear cohesive zone model. In this version, only the continuity of interfacial surface-averaged displacements and tractions is enforced, in contrast with recently developed generalized version wherein additional higherorder kinematic and static interfacial variables are also employed producing nearly perfect interfacial conformability in the absence of damage, (Cavalcante and Pindera 2014). Such capability may be important in problems undergoing very large deformations not pursued herein.

The present framework, based on the local/global stiffness matrix approach and the decomposition of interfacial displacements into continuous and discontinuous contributions, provides substantial flexibility in simulating interfacial degradation in heterogeneous materials, as well as other related phenomena. The auxiliary system of equations which governs the interfacial response admits arbitrary interfacial traction-separation laws, including CZM equations employed in the present study. Because the auxiliary equations may be activated by problem-specific conditions, various physical phenomena that may arise at different points along the loading path may be simulated using this approach. This is similar to the on-demand insertion of interface elements within the finite-element framework proposed by Ortiz and Suresh (1993) which the present framework enables naturally.

It is also significant that physically realistic interfacial parameters were identified that produced good correlation with the reported experimental response of unidirectional $\mathrm{SiC} / \mathrm{Ti}$ composite. Specifically, the employed maximum interfacial separation distance of $0.46 \mu \mathrm{m}$ corresponding to traction-free interface compares well with the SiC fiber diameter of $146 \mu \mathrm{m}$ and the thickness of the chemically-degraded fiber/matrix interfacial zone that leads to premature separation under low transverse load. This is in contrast with the wide range of interfacial parameters found in the literature, some of which may be unrealistic as reported by Chandra et al. (2002). Identification of realistic interfacial strength parameters may be accomplished by incorporating the CZM-based FVDAM theory into an optimization algorithm, as recently demonstrated by Tu and Pindera (2013) in the context of bio-inspired microstructures using the Particle Swarm Optimization of Kennedy and Eberhart (1995). Similar capability was developed in the present context in search of optimal values for the interfacial strength and stiffness by allowing these quantities to vary in the ranges $20-140 \mathrm{MPa}$ and $1,000-10,000 \mathrm{MPa} / \mu \mathrm{m}$, respectively. Optimal values of $58.5 \mathrm{MPa}$ for the interfacial strength and $1,090 \mathrm{MPa} / \mu \mathrm{m}$ for the initial interfacial stiffness were found that improved the correlation with experiment relative to 
the predictions based on the best values given in Table 4.2. Details of this combined PSOFVDAM optimization capability facilitated by the semi-analytical structure of the FVDAM theory will be discussed in Chapter 5.

\subsection{Summary}

The parametric FVDAM theory has been further extended in order to accommodate damage evolution based on displacement discontinuity functions. Auxiliary equations that represent traction conditions along specified interfaces undergoing separation, which are necessary to determine these functions, may be based either on traction-free conditions that simulate crack growth or interfacial separation laws based on the flexible interface concept. Herein, the cohesive zone model has been implemented into the auxiliary equations to simulate progressive damage between adjacent phases within the representative unit cell of a periodic material. The extended FVDAM theory was employed to study progressive damage around the fiber/matrix interface of a unidirectional $\mathrm{SiC} / \mathrm{Ti}$ material subjected to transverse loading. Consistent CZM parameters have been identified that produced very good correlation with the reported experimental response immediately after fabrication cooldown when the interface was assumed intact, as well as after several cycles which destroyed the chemical bond along a portion of the interface. Examination of the evolution of interfacial displacement discontinuities and tractions with increasing load revealed that the extended FVDAM theory with CZM-based damage evolution capability correctly captured the mechanics of progressive interfacial debonding, with the concomitant local stress fields exhibiting fidelity comparable to an elasticity solution. The study also shows unit cell architectures affect the unit cell's homogenized response and interfacial damage. In the early stage, homogenized responses generated from square and hexagonal unit cells are the same, and the difference between them increases as more severe interfacial degradation develops in the square unit cell. Further, the study illustrates that the loading direction influences the unit cell's homogenized response and interfacial damage. In the early stage, homogenized responses under transverse and through-thickness loadings are the same, and the response under through-thickness loading is more compliant because of more severe interfacial damage. 


\section{Chapter 5}

\section{Finite Volume Micromechanics-Driven}

\section{Particle Swarm Optimization}

\subsection{Introduction}

In this chapter, the newly developed CZM-based FVDAM is incorporated into the Particle Swarm Optimization (PSO) algorithm proposed by Kennedy and Eberhart (1995) to identify optimal interfacial properties used for cohesive laws in fiber-reinforced material systems. The optimization work is motivated by the observations that: 1) homogenization techniques play an important role in the development and design of engineered materials through the establishment of a direct link between function and microstructure, which includes geometry and properties, (Tu and Pindera 2013); and 2) interfacial properties play a crucial role in transferring load between different material phases and in maintaining the integrity of composite material systems, Wang and Pindera (2016). Optimization can be an effective tool to either identify parameters that can't be easily calibrated easily or to determine the optimal parameters which optimize the performance of material or structure. It is known that the cohesive parameters which control interfacial traction-separation relations are very difficult to obtain via direct experimental procedure. Very recently a sequence of papers has been published to identify cohesive parameters, such as initial interfacial stiffness $k$, interfacial strength $\sigma$ and energy release rate $G$. The general procedure to determine the interfacial parameters is to minimize the difference between experimental data of force-displacement response and numerical predictions by adjusting the interfacial parameters, (Que and Tin-Loi 2002, Lee et al. 2011, Valoroso et al. 2013, Svensson et al. 2014, Bouhala et al. 2015).

Before incorporating CZM-based FVDAM into the PSO algorithm, we first demonstrate the feasibility of this approach through a preliminary study conducted by the author to identify combinations of material and geometric parameters of wavy multilayers that best fit the experimental response of three types of porcine mitral valve chordae tendineae, namely marginal, basal and strut, that stiffen at increasingly greater stretches. This study was conducted 
by incorporating the finite-deformation based FVDAM theory without damage capability, developed by Khatam and Pindera (2012), into the PSO algorithm. The results of this investigation demonstrate that the FVDAM-driven PSO algorithm is an excellent tool to identify combinations of material and geometric parameters that minimize objective functions which may exhibit irregular features, motivating its extension to materials undergoing damage evolution. The extended CZM-based FVDAM-driven PSO algorithm is then employed to determine the interfacial properties of fiber-reinforced materials based on different unit cell types and fiber/matrix interface distributions.

\subsection{FVDAM-Driven Particle Swarm Optimization}

The semi-analytical framework of the theory facilitates implementation into higher-level analysis algorithms, such as the Particle Swarm Optimization (Kennedy and Eberhart, 1995) employed in this study. This evolutionary nongradient-based optimization algorithm successfully used in the design of flat laminates (Chen et al., 2009; Peng et al., 2011) is well-suited for problems wherein the objective function exhibits steep gradients and cusps in the design space, and hence becomes non-analytic at those points.

The PSO algorithm mimics the behavior of a swarm of birds searching for a target. The swarm consists of particles whose positions in the design variable space are employed to generate candidate solutions based on FVDAM calculations and experimental data. The position of the $i$ th particle is given by the vector $\mathbf{X}_{i}=\left(x_{1}, x_{2}, \ldots, x_{D}\right)_{i}$, where the subscript $D$ represents the number of design variables, and its velocity by $\mathbf{V}_{i}=\left(v_{1}, v_{2}, \ldots, v_{D}\right)_{i}$. During the search for an optimal solution, the particles' positions are updated based on each particle's best previous experience denoted by pBest and the best experience of other particles denoted by gBest. The updating algorithm is given by the two equations,

$$
\begin{gathered}
v_{i d}^{k+1}=\chi\left[\omega v_{i d}^{k}+a_{1} \operatorname{rand}_{1}^{k}()\left(p B e s t_{i d}^{k}-x_{i d}^{k}\right)+a_{2} \operatorname{rand}_{2}^{k}()\left(g B e s t_{i d}^{k}-x_{i d}^{k}\right)\right] \\
x_{i d}^{k+1}=x_{i d}^{k}+v_{i d}^{k+1}
\end{gathered}
$$

where the subscript $k$ represents the iteration number, $\operatorname{rand}_{1}^{k}()$ and $\operatorname{rand}_{2}^{k}()$ are random numbers with uniform distributions in the interval $[0,1], a_{1}$ and $a_{2}$ are acceleration constants. The parameter $\omega$ is the inertia weight parameter defined in terms of its initial and final values, $\omega_{\max }$ and $\omega_{\min }$, and the current and maximum iteration numbers $k$ and $k_{\max }$, 


$$
\omega=\omega_{\max }-k\left(\omega_{\max }-\omega_{\min }\right) / k_{\max }
$$

The parameter $\chi$ introduced to ensure convergence is called constriction factor, and is defined by

$$
\chi=\frac{2}{2-\varphi-\sqrt{\varphi^{2}-4 \varphi}}
$$

where $\varphi=a_{1}+a_{2}$ such that $\varphi>4$. The values of the above parameters in the present study based on previous work (Chen et al., 2009; Peng et al., 2011) are listed in Table 5.1.

In the present study, the candidate solutions are error functions which are measures of the difference between the experimental data of Johnson et al. (1990) for SiC/Ti composite under transverse loading immediately after the fabrication $555^{\circ} \mathrm{C}$ cool-down and response predicted by the CZM-based FVDAM theory. In the initial feasibility study, the experimental data was comprised of longitudinal stress-stretch responses of three types of mitral valve chordae tendineae (Liao and Vesely, 2003b) and the numerical response was generated by the finite deformation-based FVDAM theory developed by Khatam and Pindera (2012). The error functions are evaluated for each particle $\mathbf{X}_{i}$ (each set of design variables) in the following manner

$$
\operatorname{error}\left(\mathbf{X}_{i}\right)=\frac{\sum_{k=1}^{N}\left|\left(T^{F V D A M}\left(\mathbf{X}_{i}\right)\right)_{k}-\left(T^{\exp }\right)_{k}\right|}{\sum_{k=1}^{N}\left(T^{\text {exp }}\right)_{k}}
$$

at $k=1, \ldots, N$ points along the curve of $\mathrm{T}$ using experimental and predicted values. The error function plays the same role as the objective function employed in other optimization algorithms.

As the search for the optimum solution proceeds according to the flow chart given in Figure 5.1, it is possible that some of the design variables (particle positions) may become negative. Their positions are reset to the lower and upper bounds should their values for an $i$ th particle exceed these bounds at any iteration number during the search.

Table 5.1 Parameters used in the Particle Swarm Optimization algorithm.

\begin{tabular}{|c|c|c|c|}
\hline$a_{1}$ & $a_{2}$ & $\omega_{\min }$ & $\omega_{\max }$ \\
\hline 2.05 & 2.05 & 0.4 & 0.9 \\
\hline
\end{tabular}




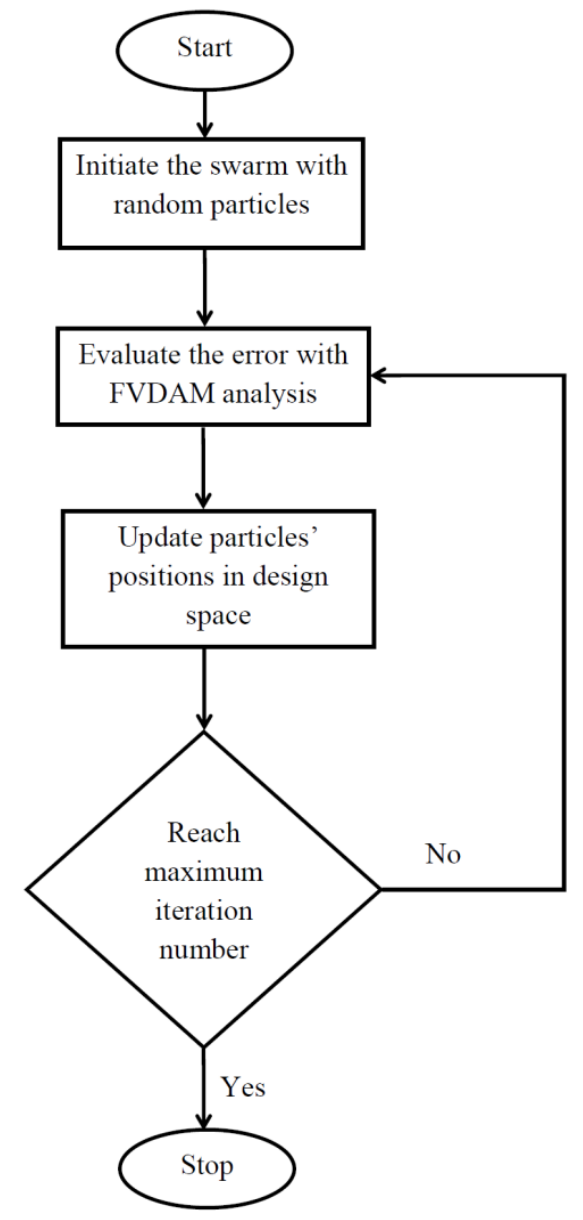

Figure 5.1 Flow chart showing the marriage of FVDAM and PSO algorithm.

\subsection{Algorithm Feasibility Study}

Before conducting optimization studies of fiber/matrix interface properties, the FVDAM-driven PSO approach is first employed to identify combinations of material and geometric parameters of wavy multilayers that best fit experimental response of three types of porcine mitral valve chordae tendineae, namely marginal, basal and strut, that stiffen at increasingly greater stretches. The employed FVDAM theory developed by Khatam and Pindera (2012) has the capability to analyze finite deformation problems but not problems involving damage evolution.

\subsubsection{Model of the Mitral Valve Chordae Tendineae}

Following the previous investigation (Khatam and Pindera, 2012), the complex microstructure of porcine mitral valve chordae tendineae is represented by a wavy multilayer unit cell. The 
construction of the simplified model for simulating the chordae response is based on the reported experimental data for this biological tissue, which includes the amplitude and wavelength ranges of the crimped collagen fibril bundles and Young's modulus ranges of the limiting tensile response of the marginal, basal and strut chordae (Liao and Vesely, 2003a). These are reproduced in Table 5.2. The amplitude ranges are based on SEM observations, while a polarized light technique had been used to determine the wavelength ranges. The homogenized tensile response had been generated in an Instron machine by first preconditioning the chordae at $4 \mathrm{~mm} / \mathrm{s}$ to $150 \mathrm{~g}$ load in a bath of Hank's solution to simulate physiological environment until the loaddisplacement curve became repeatable, which was followed by the actual test in the elastic range. Figure 5.2 summarizes the stabilized elastic stress-strain responses of the three chordae types (reproduced from Liao and Vesely, 2003b) which will be employed for comparison with FVDAM-based simulations.

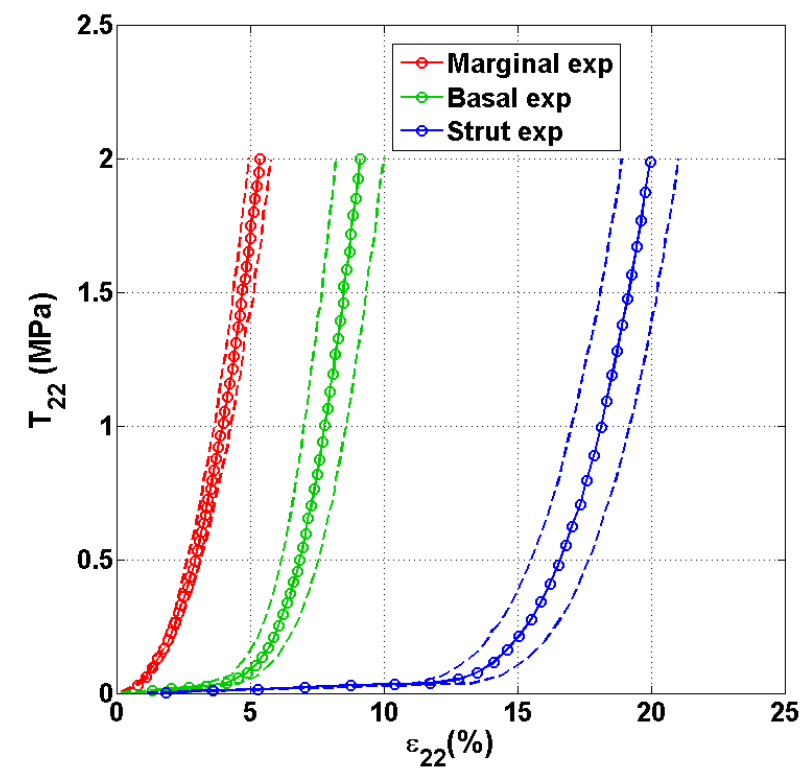

Figure 5.2 Longitudinal stress-strain response of porcine mitral valve marginal, basal and strut chordae (reproduced by digitization from the experimental data of Liao and Vesely, 2003b).

The above information is used to construct unit cells, comprised of alternating wavy stiff and soft layers, Figure 5.3, to target the actual response of marginal, basal and strut chordae tendineae. Such a model is obviously a simplification of the actual crimped geometry of chordae collagen fibril bundles, but the goal herein is to show that it may be successfully used to simulate the actual tensile responses of porcine mitral valve chordae tendineae upon identification of optimal geometric and material parameters. In the wavy multilayer model, the stiff phase 
represents the average behavior of the crimped fibril bundles, and the soft phase the ground substance, with the respective volume fractions representative of the actual biological tissue. The amplitude and wavelength of the unit cell are varied according to the experimental data in Table 5.2. The thickness of the reference stiff layer, $0.42 \mu \mathrm{m}$, is the same as in the previous study (Khatam and Pindera, 2012). Given that the fibril diameter within fibril sub-bundles of the crimped marginal, basal and strut chordae lies in the range 45-60 $\mathrm{nm}$ (Liao and Vesely, 2003a), the thickest stiff layer corresponds to about 7-9 fibril diameters. The overall thickness of the unit cell is adjusted such that the overall stiff layer volume fraction is fixed at 0.48 as reported (Liao and Vesely, 2003a), with the length dependent on the crimp period. The microstructural refinement of the reference unit cell is attained by subdividing the thick stiff layer into progressively thinner ones, with the maximum number of subdivisions producing 8 stiff layers and the thinnest stiff layer thickness on the order of a single fibril diameter.

While the previous study (Khatam and Pindera, 2012) was limited to the effect of soft layer modulus and stiff layer thickness in the simulation of porcine mitral valve strut chordae using mean values of the reported amplitude-to-wavelength ratio of the crimped fibril microstructure and the Young's modulus of the limiting chordae response, the present verification work is much broader. It includes the effects of variations of amplitude, period, soft and stiff layer Young's moduli as well as microstructural refinement for all three chordae models.

Table 5.2 Ranges of the amplitude and wavelength of the crimped microstructure of the porcine mitral valve marginal, basal and strut chordae tendineae and the Young's modulus of the limiting linear response (Liao and Vesely, 2003a) employed in the construction of unit cell models and the extraction of hyperelastic constitutive model parameters.

\begin{tabular}{|l|l|l|l|}
\hline Chordae Type & Amplitude $(\mu m)$ & Wavelength $(\mu m)$ & $E_{\text {Chordae }}(\mathrm{MPa})$ \\
\hline Marginal & $1.87 \pm 0.78$ & $14.8 \pm 3.0$ & $84.4 \pm 21.2$ \\
\hline Basal & $1.87 \pm 0.78$ & $14.8 \pm 3.0$ & $86.1 \pm 20.9$ \\
\hline Strut & $1.87 \pm 0.78$ & $11.3 \pm 1.4$ & $64.2 \pm 13.5$ \\
\hline
\end{tabular}



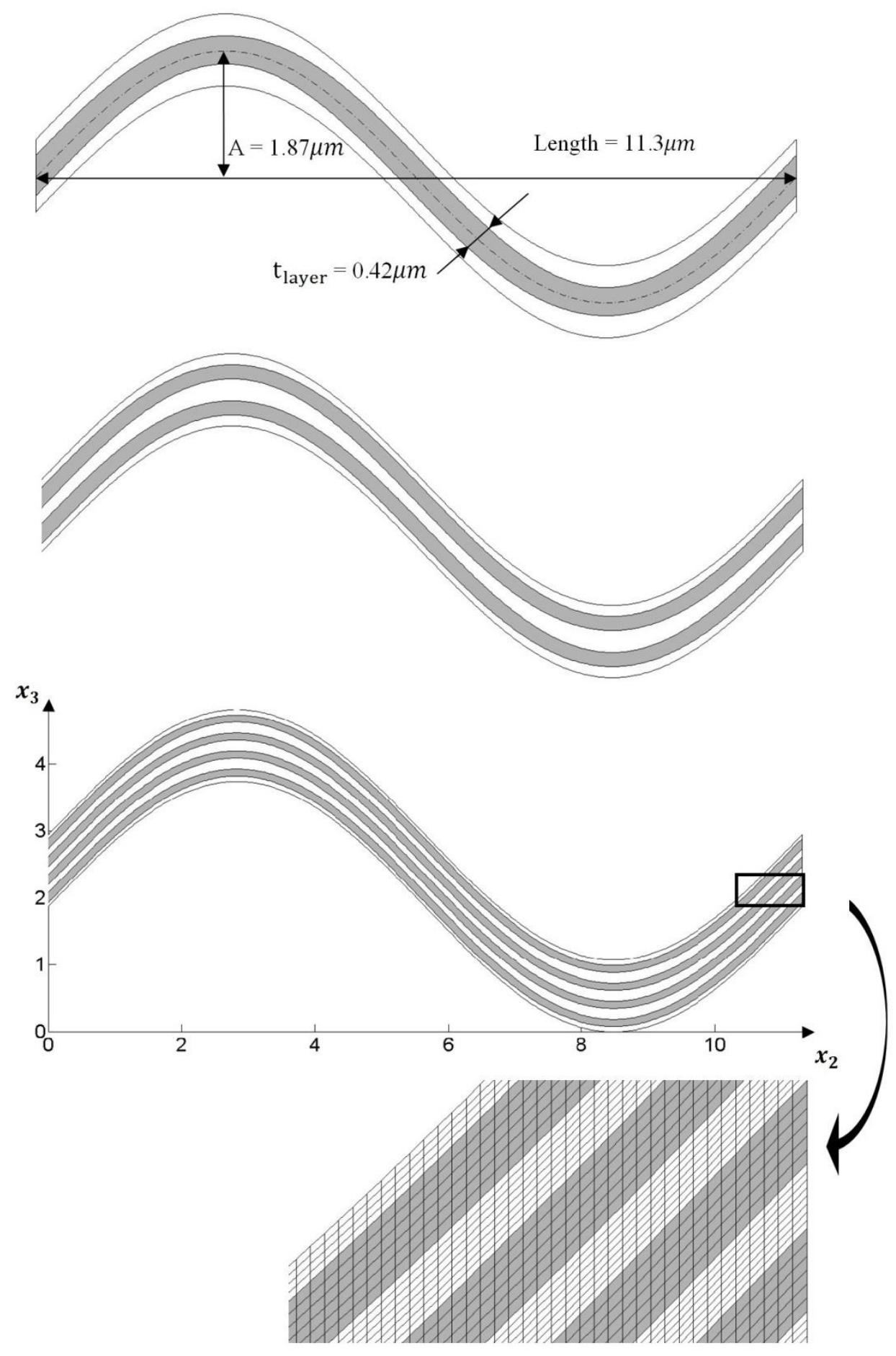

Figure 5.3 Geometry of the simplified model of the microstructure of porcine mitral valve strut chordae employed in the FVDAM simulations based on the reported mean values of amplitude and crimp period (Liao and Vesely, 2003a). The top representative unit cell is the reference cell with thickest stiff layer which is further subdividing in a manner that retains its volume fraction (middle and bottom unit cells). Detail of the unit cell discretization is shown in the bottom unit cell (note: axes scale is in microns). 


\subsubsection{Unit Cell Discretization}

The reference unit cell with a single stiff layer for the marginal and basal chordae is generated using mesh discretization of $468 \times 24$ quadrilateral subvolumes along the horizontal and vertical directions, respectively. This discretization ensures that the aspect ratio of each subvolume is not excessively large. Similarly, the reference unit cell for the strut chordae is discretized into $312 \times 24$ subvolumes due to smaller crimp period. Microstructural refinement is accomplished in a manner that ensures that the overall unit thickness, stiff layer volume fraction and amplitudeto-wavelength ratio remain fixed. In this case, the smallest unit cell for the given refinement is employed. For instance, the smallest unit cell for the marginal and basal chordae with the thick stiff layer subdivided into 8 thinner ones contains a single thin stiff layer which requires the discretization of $1080 \times 14$ to maintain a reasonable subvolume aspect ratio. A full unit cell containing 8 thin stiff layers would be discretized into $1080 \times 112$ subvolumes. The corresponding discretization for the smallest strut chordae unit cell containing one thin stiff layer is $720 \times 14$ in contrast with $720 \times 112$ subvolumes for the full unit cell with 8 thin stiff layers.

\subsubsection{Phase Constitutive Response}

Both the stiff and soft phases of the wavy multilayer model, that represent collagen fibril bundles and ground substance material with complicated microstructure comprised of randomly oriented network of collagen filaments (elastin) embedded in a hydrated proteoglycan gel producing soft and isotropic matrix, are represented by the generalized Mooney-Rivlin material. The strain energy density function for this material model is given in terms of the three invariants of the right-Cauchy deformation tensor $\mathbf{C}, I_{1}=\operatorname{tr} \mathbf{C}, I_{2}=\frac{1}{2}\left(\operatorname{tr}^{2} \mathbf{C}-\operatorname{tr} \mathbf{C}^{2}\right), I_{3}=\operatorname{det} \mathbf{C}$,

$$
W=c_{1}\left(\frac{I_{1}}{I_{3}^{1 / 3}}-3\right)+c_{2}\left(\frac{I_{2}}{I_{3}^{2 / 3}}-3\right)+\frac{\kappa}{2}(J-1)^{2}
$$

where $=\operatorname{det} \mathrm{F}=\sqrt{I_{3}}, c_{1}$ and $c_{2}$ are material parameters with $c_{1}+c_{2}=\mu / 2$, and $\mu$ and $\kappa$ are shear and bulk moduli, respectively. The compressibility or Poisson's ratio may be changed by assigning different values for $\kappa=2 \mu(1+v) / 3(1-2 v)$. This hyperelastic constitutive model is often used for soft materials because of the control of the amount of compressibility.

The Young's modulus ranges of the stiff layers, which represent the crimped bundles of collagen fibrils in the considered mitral valve marginal, basal and strut chordae tendineae, are 
determined from the limiting linear response of the experimentally determined chordae stressstrain curves, Table 5.2. The individual fibrils within the fiber bundles are assumed to have straightened out when the chordae stiffening response becomes linear. Using the chordae Young's modulus ranges listed in Table 5.2, the volume fraction of collagen fibrils of 0.48 , and the rule-of-mixtures formula based on the assumption of negligible contribution from the ground substance, the Young's modulus ranges of the stiff layers in the simplified wavy model are thus obtained and listed in Table 5.3. The knowledge of the Young's moduli, together with an assumed Poisson's ratio of 0.30 allows one to calculate the initial shear modulus ranges from the isotropic relation $\mu=E / 2(1+v)$ and hence the bulk modulus ranges. Taking $\mathrm{c}_{2}=0$ as in the previous study (Khatam and Pindera, 2012), which in fact reduces the generalized MooneyRivlin material to a compressible neo-Hookean one, all the required material parameters needed in Eq. (5.5) are obtained. The stiff layer parameter ranges thus extracted from the marginal, basal and strut chordae response are included in Table 5.3.

The following assumption and approximation intrinsic to the above calculations need to be highlighted. The stiff layers in the wavy multilayer model are taken as isotropic, while the microstructure of collagen fibrils suggests, at least, transversely isotropic properties. However, the soft layers representing the ground substance are not expected to significantly affect the transverse deformation of the stiff layers under longitudinal loading because the major stress component controlling the unfolding and stiffening of the wavy microstructure is along the applied load. Hence the transverse properties of the stiff layers will have a minimal influence on the axial deformation of the chordae which is the focus in the present study. Clearly, this model will not be accurate under multiaxial loading of the considered chordae tendineae which would require accurate transverse fibril properties to be realistic. Hence the extraction of the constituent phase properties in the manner described above limits the model to longitudinal loading, the primary loading for the considered tissue. This circumvents the issue of incompressibility assumed for many biological tissues under multiaxial loading, and supports the choice for Poisson's ratio values of the stiff and soft layers representative of many polymers. 
Table 5.3 Elastic parameters of the generalized Mooney-Rivlin model extracted from the experimental data on the porcine mitral valve marginal, basal and strut chordae tendineae (Liao and Vesely, 2003a) employed in the simplified unit cell models for the response of chordae tendineae constituents. The marginal, basal and strut layers are the stiff layers in the simplified model and the matrix is the soft layer.

\begin{tabular}{|l|c|c|c|c|c|}
\hline Material & $\mathrm{E}(\mathrm{MPa})$ & $v$ & $\kappa(\mathrm{MPa})$ & $\mathrm{c}_{1}(\mathrm{MPa})$ & $\mathrm{c}_{2}(\mathrm{MPa})$ \\
\hline Marginal layer & $175.83 \pm 44.16$ & 0.30 & $146.53 \pm 36.81$ & $33.81 \pm 8.49$ & 0.0 \\
\hline Basal layer & $179.37 \pm 43.54$ & 0.30 & $149.48 \pm 36.28$ & $34.49 \pm 8.37$ & 0.0 \\
\hline Strut layer & $133.75 \pm 28.12$ & 0.30 & $111.46 \pm 23.44$ & $25.72 \pm 5.41$ & 0.0 \\
\hline Matrix & 0.1 & 0.30 & 0.083 & 0.019 & 0.0 \\
\hline
\end{tabular}

\subsubsection{Targeting the Response Using Particle Swarm Optimization}

In this section, we present detailed results of optimization studies using fixed amplitude and wavelength values identified in the initial sensitivity and optimization studies that minimize the error in the predicted homogenized response for the three chordae types. The amplitudes are $1.09,1.48$ and $1.675 \mu \mathrm{m}$ for the marginal, basal and strut chordae models, respectively, with the corresponding wavelengths set at 17.8, 17.8, and $12.35 \mu \mathrm{m}$. Thus the design variables for the three chordae models are the number of stiff layers in the range $[1,8]$ at fixed volume fraction, the matrix modulus $E_{m}$ which lies in the range $[0.05,1.25] \mathrm{MPa}$, and the stiff layer modulus $E_{f}$ with ranges dependent on the chordae. For the marginal chordae the range of $E_{f}$ is $[130,220]$ $\mathrm{MPa}$, while for the basal and strut chordae the respective ranges are [136, 223] MPa and [106, 162] MPa. The objective function in all cases is the previously defined error that minimizes deviation of the predicted response from the experimental data (Liao and Vesely, 2003b). The number of particles used in the optimization runs was 12 and the number of allowed iterations was 14, which was sufficient to obtain converged results. Figure 5.4 illustrates the error convergence as a function of iteration number for the three chordae models. Detailed results follow.

Figure 5.5 illustrates the results of the optimization process for the marginal chordae model, which include the initial and final particle distributions in the three-dimensional design variable space, and the initial and converged homogenized responses after 10 iterations. The error decreases from an initial value of 0.080 to the final value of 0.036 with the concomitant design variable changes from $E_{m}=0.959, E_{f}=197.0$ and 6 stiff layers to the final values of $E_{m}=0.912, E_{f}=219.2$ and 8 stiff layers. We note that each set of results represents the design variables that produce the best solution of the entire twelve-particle swarm for the given 
iteration. The above results compare well with the results of the sensitivity studies followed by a more intuitive procedure that produced the best homogenized response for the set of design variables given by $E_{m}=0.75$ and $E_{f}=219.58 \mathrm{MPa}$ and 4 stiff layers.

Figures 5.6 and 5.7 present the corresponding results for the basal and strut chordae models, respectively. For the basal chordae model, only 5 iterations are required to obtain converged homogenized response that minimizes the error relative to the experimental data, with the initial error of 0.11 after the first iteration decreasing to 0.0223 . The swarm of particles with an initially random distribution in the three-dimensional design variable space converges to a very small cluster upon final iteration, Figure 5.6a. The best of the initial set of design variables after the first iteration, comprised of $E_{m}=0.088, E_{f}=159.95$ and 6 stiff layers, produces a slightly stiffer initial and a more compliant limiting response relative to the experimental data. Reduction and increase in the matrix and stiff layer moduli, respectively, yields an optimal set of design variables with the best solution comprised of $E_{m}=0.05, E_{f}=185.14$ and 7 stiff layers which yield an excellent agreement with the experimental data, Figure 5.6b. These values compare well with those obtained from the parametric sensitivity studies based on the optimal amplitude and wavelength identified for the basal chordae model which yielded $E_{m}=0.05$, $E_{f}=179.37 \mathrm{MPa}$ with the number of stiff layers varying between 3 to 8 .

The initial and final design variable distributions and the corresponding homogenized responses for the strut chordae model are shown in Figure 5.7. The best of the initial set of design variables whose distribution is shown in Figure 5.7a produces an error of 0.145 based on the values of $E_{m}=0.115, E_{f}=135.48$ and 2 stiff layers. This initial set does not capture well enough the initially compliant response, Figure $5.7 \mathrm{~b}$, owing to the large bending stiffness and hence resistance of the relatively thick stiff layers during the unfolding process. The final particle swarm converges to a small cluster in the design variable space with the best set of values given by $E_{m}=0.05, E_{f}=147.51 \mathrm{MPa}$ and 8 stiff layers. These values produce and error of 0.0377 between the predicted homogenized response and experimental data which exhibit excellent correlation. They also compare well with those obtained from the parametric sensitivity studies which yielded $E_{m}=0.05, E_{f}=133.75-161.00 \mathrm{MPa}$ with the number of stiff layers varying between 4 to 8 . 
From the above study we can conclude that the homogenization-based Particle Swarm Optimization algorithm is an excellent tool in identifying optimal unit cell architectures in the design space that exhibits very steep gradients. The demonstrated feasibility of the FVDAMdriven PSO approach will be employed in the following section to optimize the CZM parameters in order to simulate the response of $\mathrm{SiC} / \mathrm{Ti}$ unidirectional composites with evolving degradation of the fiber/matrix interface.

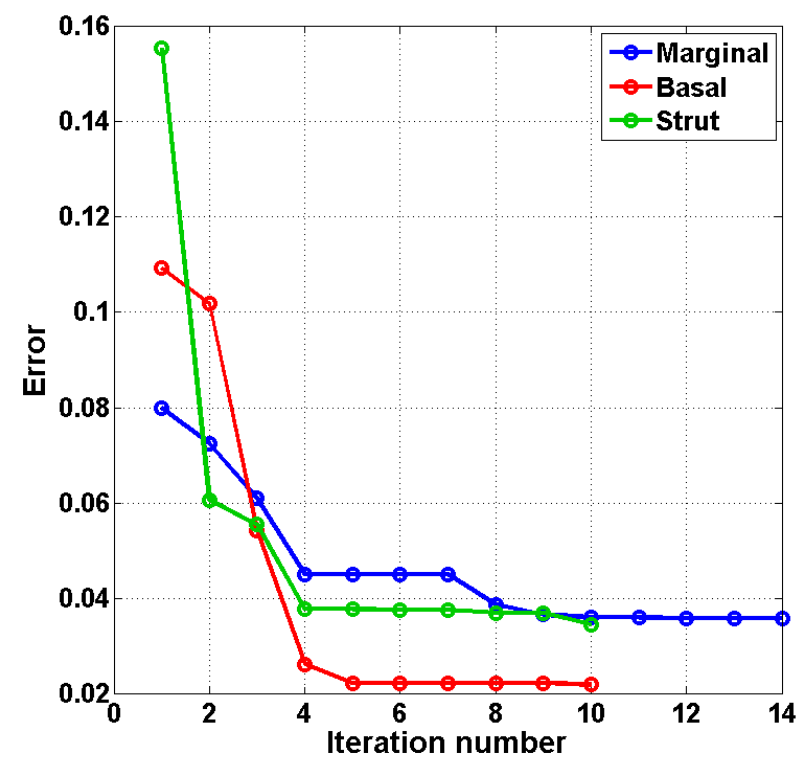

Figure 5.4 The error convergence with iteration number of the PSO algorithm in simulating the homogenized response of porcine mitral valve marginal, basal and strut chordae.

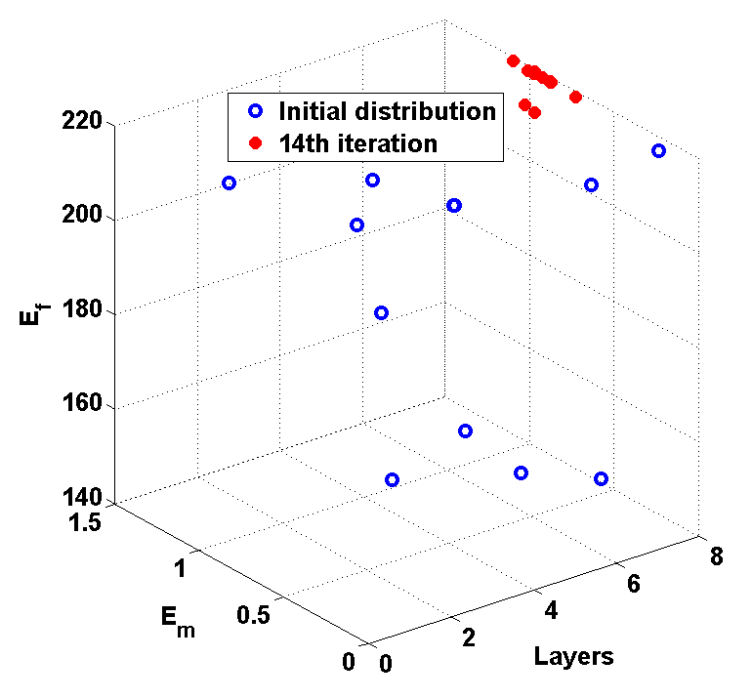

(a)

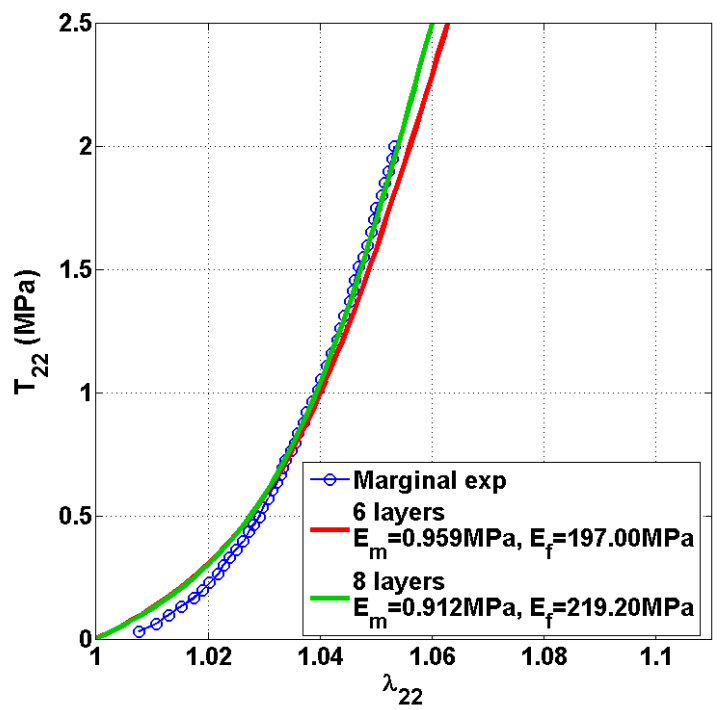

(b)

Figure 5.5 (a) Initial and final particle distributions. (b) Best initial and final simulated homogenized responses of the marginal chordae tendineae. 


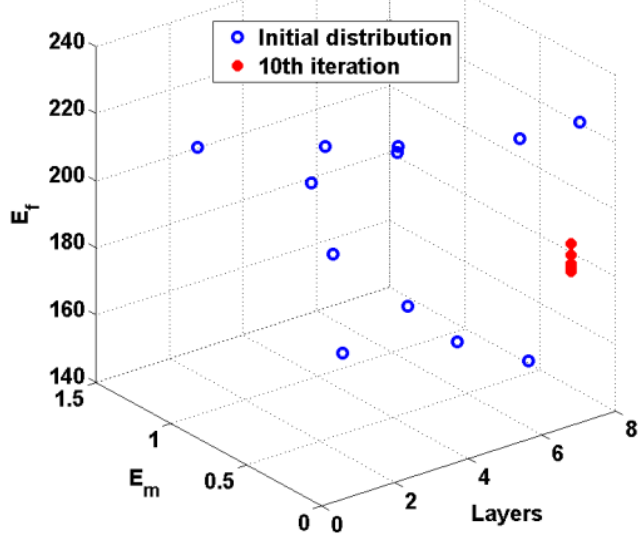

(a)

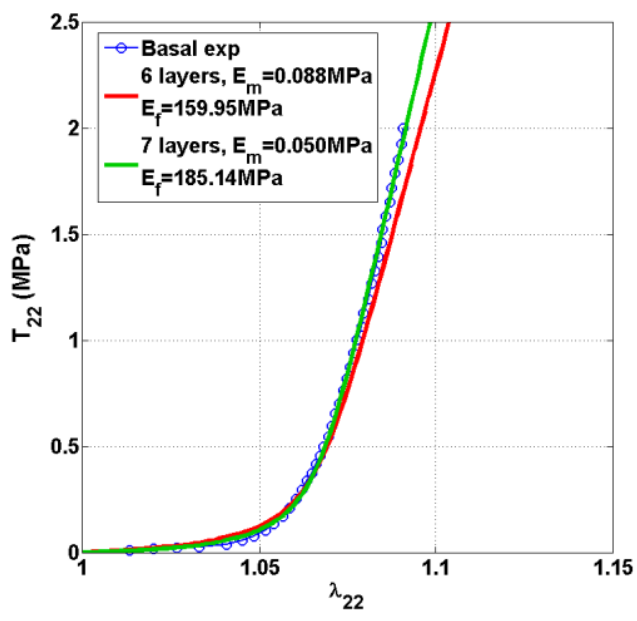

(b)

Figure 5.6 (a) Initial and final particle distributions. (b) Best initial and final simulated homogenized responses of the basal chordae tendineae.

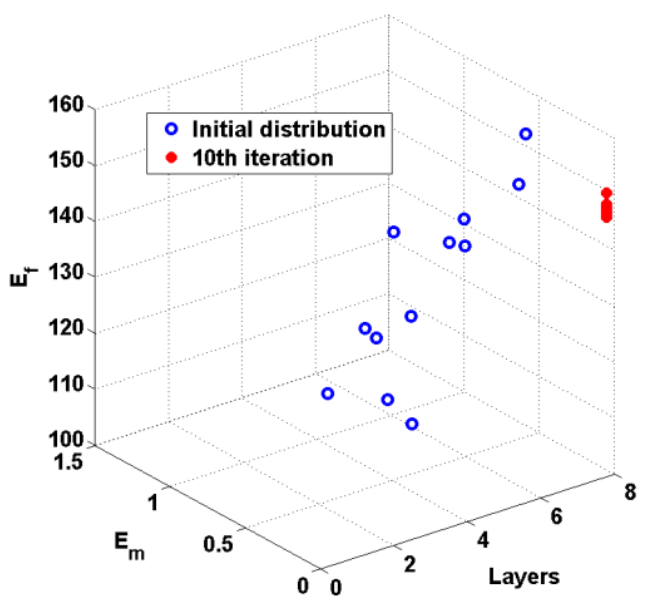

(a)

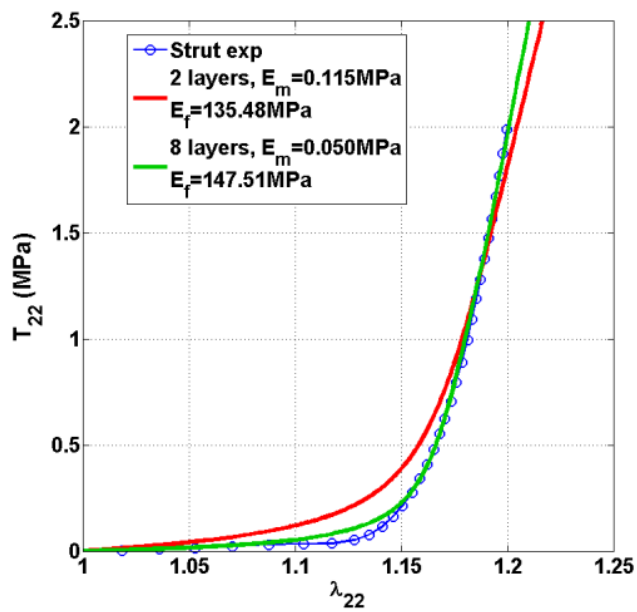

(b)

Figure 5.7 (a) Initial and final particle distributions. (b) Best initial and final simulated homogenized responses of the strut chordae tendineae.

\subsection{Optimizing CZM Parameters for Interfaces Undergoing Damage}

\subsubsection{Interfacial Parameter Optimization based on Square Unit Cell}

In Chapter 4, the best correlation with experimental data of Johnson et al. (1990) for a SiC/Ti composite under transverse loading immediately after the fabrication $555^{\circ} \mathrm{C}$ cool-down was obtained using a trial-and-error procedure by first varying the initial interfacial stiffness and then 
interfacial strength with the initial stiffness fixed. Using this parametric approach with the uncoupled bilinear cohesive law, the best fit to experimental data was obtained for the initial interfacial stiffness of $3 \times 10^{3} \mu \mathrm{m} / \mathrm{MPa}$ and interfacial strength of $50 \mathrm{MPa}$ ( $\mathrm{Tu}$ and Pindera, 2014).

Here, FVDAM-driven optimization is employed to obtain the best interfacial stiffness and interfacial strength. We first verify the optimization algorithm against the results of the above parametric study by fixing the initial interfacial stiffness at $3 \times 10^{3} \mu \mathrm{m} / \mathrm{MPa}$ and using the interfacial strength as a design variable in the range of [20 140] MPa with 10 particles and 20 iterations. The optimal interfacial strength was identified to be $48.9 \mathrm{MPa}$, which agrees well with the value obtained from the parametric study, namely $50 \mathrm{MPa}$, with the objective function converging to 0.0412 . Figure 5.8a shows the convergence behavior of the objective function, illustrating that after 12 iterations optimum interfacial strength that minimizes the objective function is obtained. Figure 5.8b shows the comparison between experimental data and optimum response from the parametric study of Tu and Pindera (2014) and current optimization, both of which agree well with each other and with experiment.

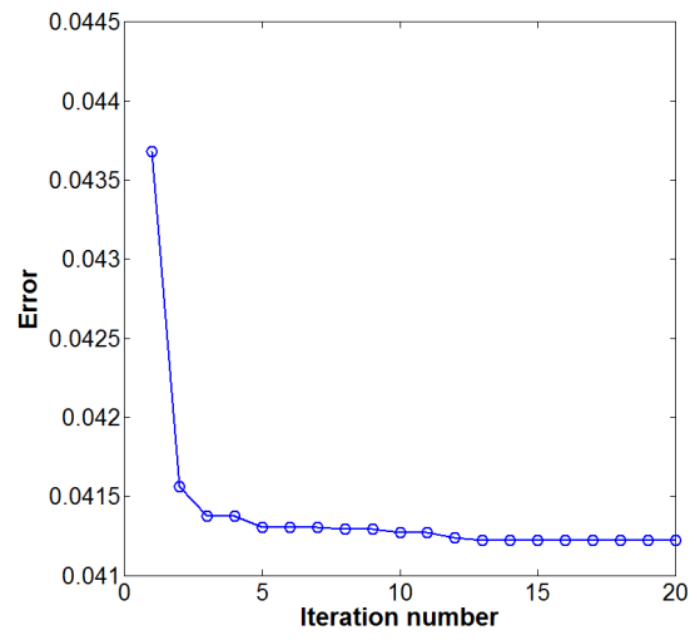

(a)

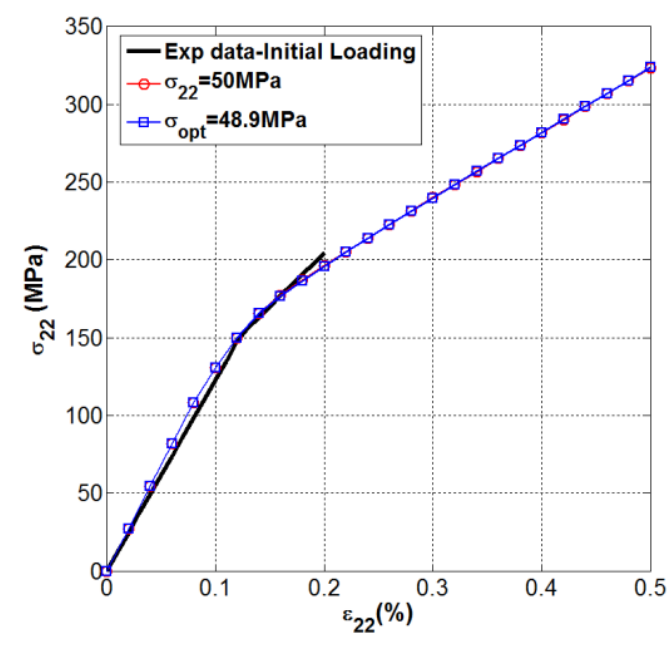

(b)

Figure 5.8 (a) Optimization convergence curve; (b) Comparison between experimental data and optimized homogenized response.

Increasing the design variable space to include both the initial interfacial stiffness and strength leads to the identification of optimum design variables that further improve correlation with experiment. The two design variables are chosen in the ranges $\left[0.5 \times 10^{3} 10 \times\right.$ $\left.10^{3}\right] \mu \mathrm{m} / \mathrm{MPa}$ and [20 140] MPa for the initial interfacial stiffness and strength, respectively. 
Figure 5.9a illustrates the convergence of the error with iteration number, showing that after 8 iterations the objective or error function reaches a small value and the minimum value is obtained after the 10th iteration before the maximum step in the analysis is reached. Figure 5.9b illustrates particle distributions at the first and final steps and we observe that at the final step the particles converge to a single point in the two-dimensional design space. Figure 5.10 illustrates the comparison between experimental data, optimized homogenized response and the best fit obtained from the parametric study. The optimized response actually shows better agreement with the experimental data than the best case from the parametric study. Moreover the optimization results are obtained with much less effort than that expanded in conducting the parametric studies.
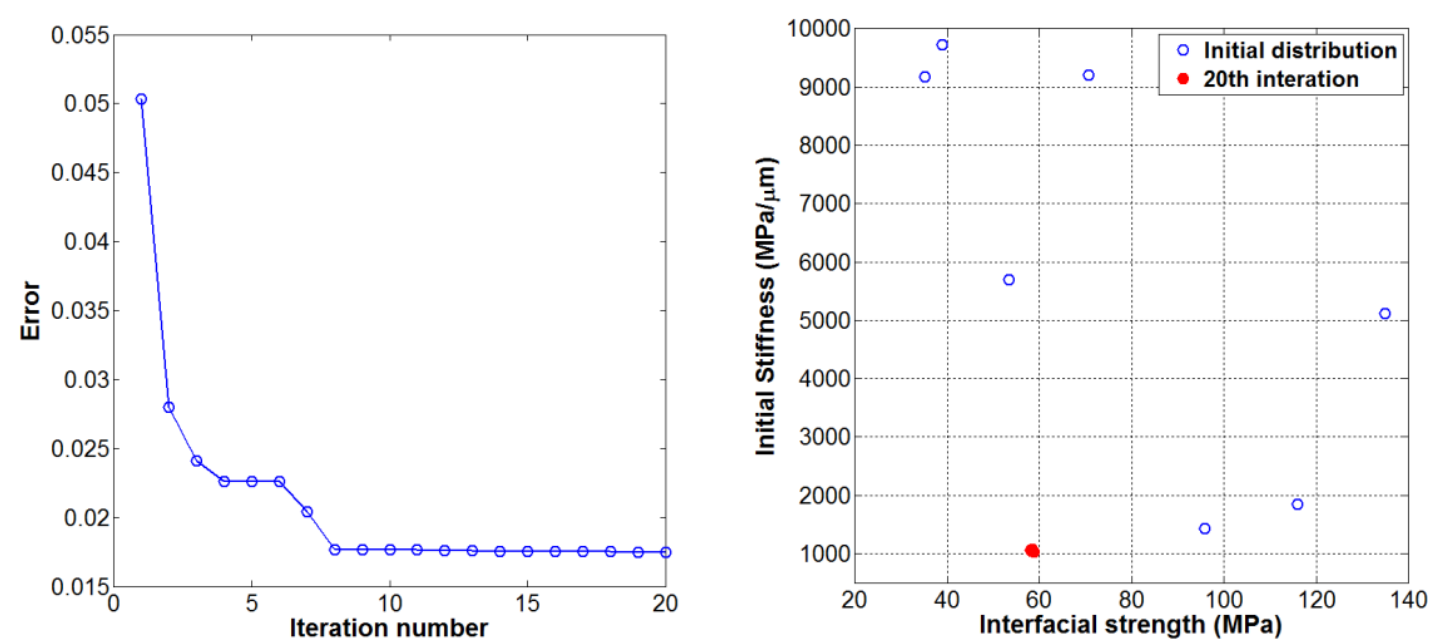

Figure 5.9(a) Optimization convergence curve; (b) Initial particle distribution and final particle distribution.

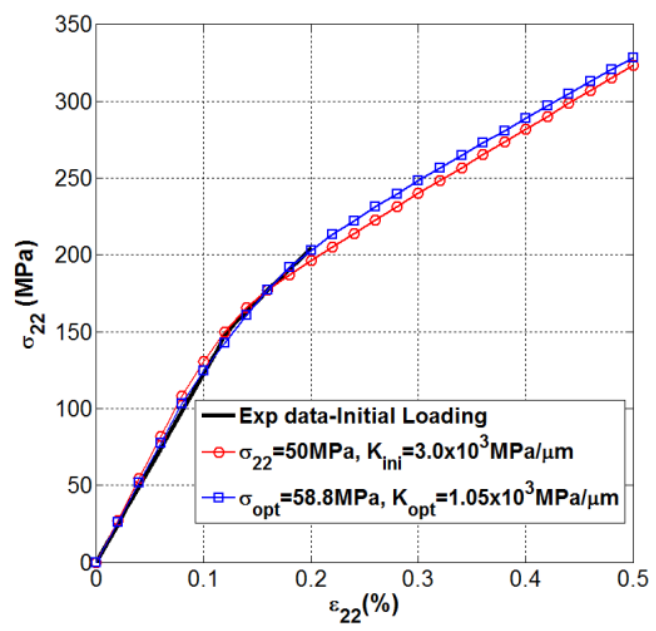

Figure 5.10 Comparison between experimental data and optimized homogenized response. 


\subsubsection{Effect of Randomness of Active Interfaces on Optimization}

It is known that the high consolidation temperature used to fabricate the $\mathrm{SiC} / \mathrm{Ti}$ material system produces a chemical reaction at the fiber/matrix interface which results in a substantially degraded chemical bond, leading to premature fiber/matrix interfacial debonding at low transverse normal stresses. However, since the consolidation condition for each fiber and adjacent matrix may not be exactly the same, some fiber/matrix interfaces may be consolidated better with less damage than others, leading to debonding at a later stage or not at all because of the shielding effect. Hence in this section, the effect randomness of active fiber/matrix interfaces on the identification of optimum CZM parameters is investigated. Figure 5.11 shows three types of fiber/matrix interface distributions with different percentage of active interfaces, which can experience damage. In the first scenario, all fiber/matrix interfaces are active and therefore may debond, Figure 5.11a. In the second scenario, the percentage of active interfaces is $75 \%$ obtained by de-activating the central fiber/matrix interfaces in the hexagonal array of fibers, Figure 5.11b. In the third scenario, the percentage of active interface is $50 \%$ with two fiber/matrix interfaces de-activated, Figure 5.11c.

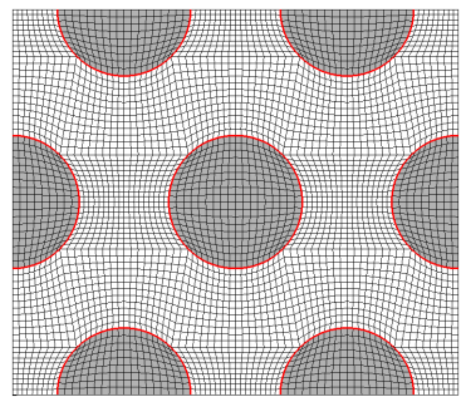

(a)

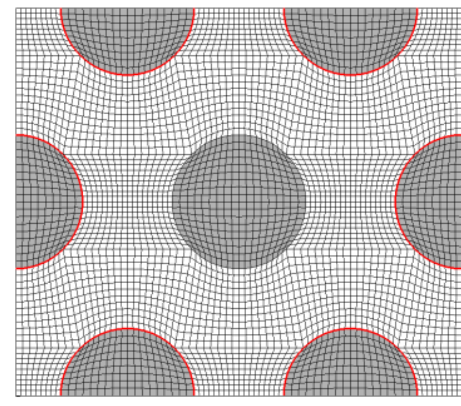

(b)

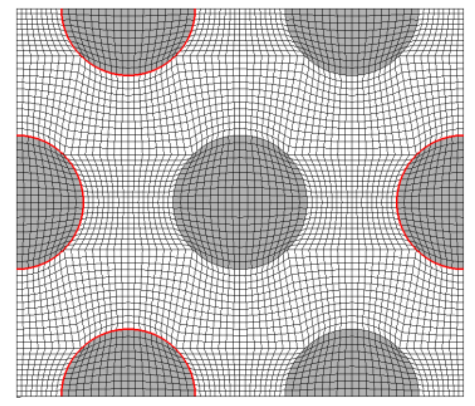

(c)

Figure 5.11 Active fiber/matrix interface distribution in a hexagonal unit cell: (a) 100\% active interface; (b) 75\% active interface; (c) 50\% active interface.

In the optimization procedure, the design variables are the interfacial strength and interfacial stiffness and they are allowed to vary in the range of [20 140] MPa and $\left[0.5 \times 10^{3}\right.$ $10 \times 10^{3} \mathrm{JPa} / \mu \mathrm{m}$. The objective is to minimize the difference between the homogenized response and the experimental data for $\mathrm{SiC} / \mathrm{Ti}$ under transverse loading immediately after the fabrication $555^{\circ} \mathrm{C}$ cool-down. Figure 5.12 shows the convergences behavior with iteration number for the three types of interface distributions shown in Figure 5.11. We observed that for two cases convergence occurs after 12 iterations, with one case converging after only 3 
iterations. For the cases (a) and (b), the smallest difference between experimental response and simulation data is 0.015 and for the case (c), the smallest difference between experimental response and simulation data is 0.03. Table 5.4 lists the corresponding optimized design variables for those three cases. We note that the case (c) has the smallest interfacial strength, which also has the least active fiber/matrix interface, because more damage needs to be introduced to the active fiber/matrix interface to match the experimental response. Figure 5.13 shows the particle distributions after 20th iterations for the three cases. We see that almost all particles converge to the optimal values, which are $\left(59.1 \mathrm{MPa}, 1.33 \times 10^{3} \mathrm{MPa} / \mu \mathrm{m}\right)$, $\left(48.7 \mathrm{MPa}, 0.86 \times 10^{3} \mathrm{MPa} / \mu \mathrm{m}\right)$ and $\left(33.4 \mathrm{MPa}, 9.95 \times 10^{3} \mathrm{MPa} / \mu \mathrm{m}\right)$ for the three cases (a), (b) and (c), respectively.

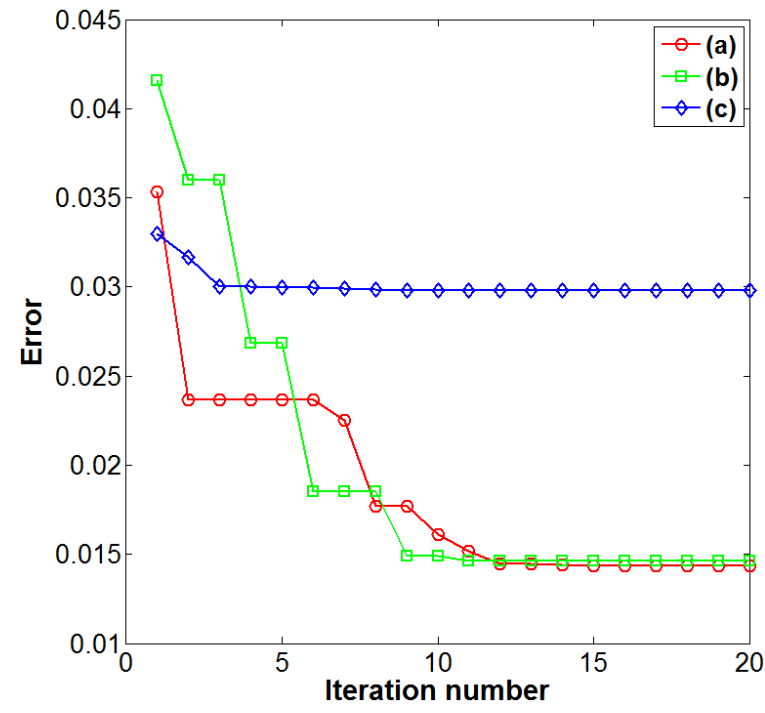

Figure 5.12 Convergence responses for three types of interface distributions as shown in Figure 5.11 .

Table 5.4 Optimized design variables for three types of interface distributions.

\begin{tabular}{|c|c|c|c|}
\hline & Percentage of active interfaces & $\sigma_{\max }(\mathrm{MPa})$ & $K(\mathrm{MPa} / \mu \mathrm{m})$ \\
\hline $\mathrm{a}$ & $100 \%$ & 59.1 & $1.33 \times 10^{3}$ \\
\hline $\mathrm{b}$ & $75 \%$ & 48.7 & $0.86 \times 10^{3}$ \\
\hline $\mathrm{c}$ & $50 \%$ & 33.4 & $9.95 \times 10^{3}$ \\
\hline
\end{tabular}




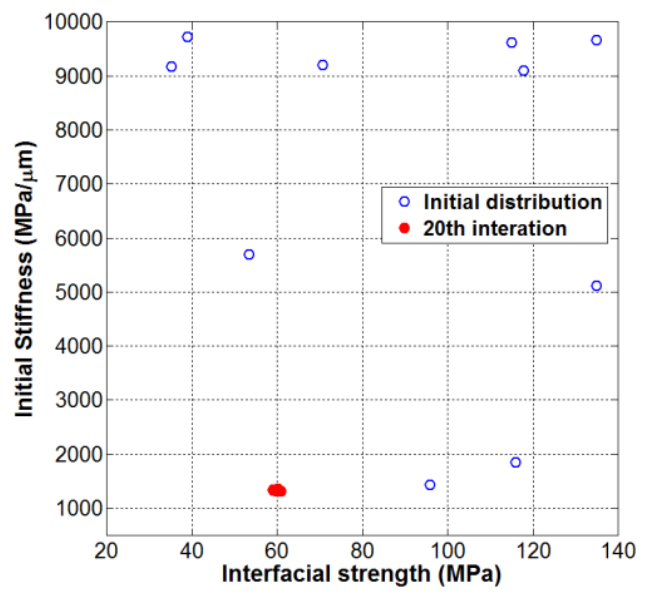

(a)

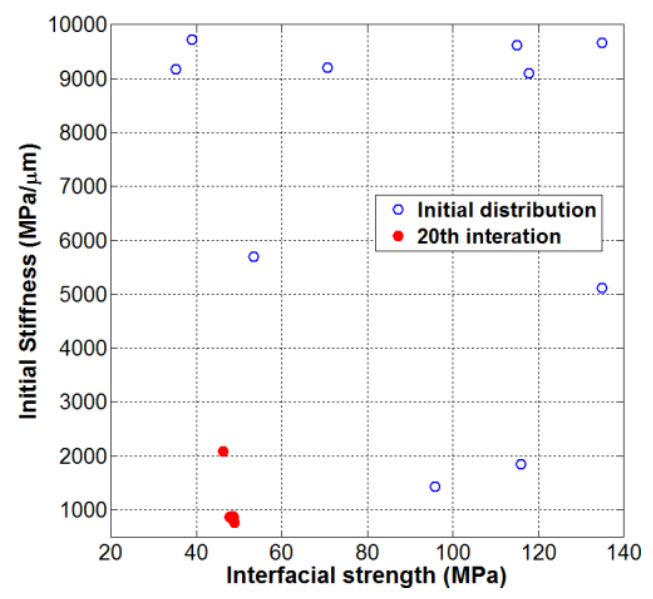

(b)

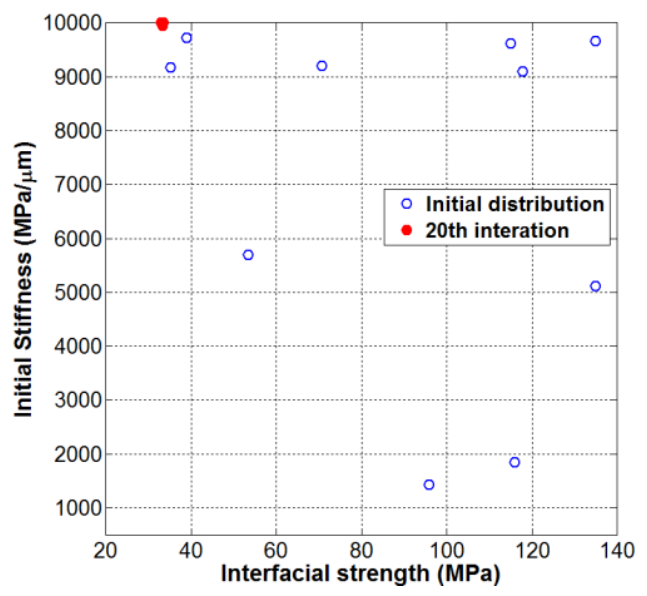

(c)

Figure 5.13 Particles' distributions for case (a), (b), (c).

Figure 5.14 compares the homogenized responses for the three types of interface distributions with the optimized design variables listed in Table 5.4. The homogenized responses agree very well with the experimental data, which terminates at the strain level of $0.2 \%$. However, when the applied strain is higher than $0.2 \%$ the three responses diverge, with the homogenized response of case (a) being the most compliant one and the homogenized response of case (c) the stiffest one. When the applied strain level is low, the case with smaller percentage of active interfaces has lower interfacial strength in order to target the experimental response because more damage needs to introduced into each active fiber/matrix interface compared with the case with higher percentage of active interfaces. With the increase in loading, interfacial strength become less important than the number of active fiber/matrix interface. Hence case (a) 
is the most compliant one and case (c) is the stiffest one. The homogenized responses of those three cases are illustrated via the full-field stress distributions in Figures 5.15-5.16.

Figure 5.15 shows the stress distributions for the three cases at step 10. In case (a), all interfaces are at a similar interfacial damage stage, at which the fiber/matrix interface perpendicular to the loading direction degrades and stress concentration arises in the matrix phase above and below the fiber. In cases (b) and (c), the stress inside the fiber surrounded by perfectly bonded interface is higher than the stress in the fiber surrounded by an active fiber/matrix interface and the corresponding stress concentration in the matrix phase above and below the fiber is much less severe. Although in case (a) all fiber/matrix interfaces experience degradation in contrast with cases (b) and (c) in which some interfaces remain intact, the homogenized responses are the same at the applied strain of $0.2 \%$ as shown in Figure 5.14. This is because in the early degradation stage the interfacial strengths of cases (b) and (c) are smaller than in case (a) and hence experience greater interfacial damage, which is reflected in more severe contrast in the normal stress $\sigma_{22}$.

Figure 5.16 shows the stress distributions for the three types of interface distributions at step 20 which corresponds to the homogenized stress $\sigma_{22}$ of $280 \mathrm{MPa}$ for case (a) and $305 \mathrm{MPa}$ and 333MPa for cases (b) and (c), respectively. The lower homogenized stress in case (a) is due to all interfaces experiencing degradation which causes more total loss of load transfer capability between matrix and fiber. In contrast, in cases (b) and (c) some fiber/matrix interfaces are intact, and thus the loss of load transfer capability is less dramatic. This is observed in the $\sigma_{22}$ stress distributions which exhibit relatively high stress magnitudes in both fiber and matrix phases. Also it should be noticed that the interface distributions alter the stress symmetry. In cases (a) and (b) the symmetrical $\sigma_{22}$ and $\sigma_{33}$ normal stress distributions and the anti-symmetrical $\sigma_{23}$ shear stress distributions are maintained because of the symmetrical interface distribution. In case (c), however, $\sigma_{22}$ and $\sigma_{33}$ distributions no longer remain symmetric, and $\sigma_{23}$ stress distribution does not exhibit anti-symmetry with respect to the vertical plane across the center of the middle fiber. 


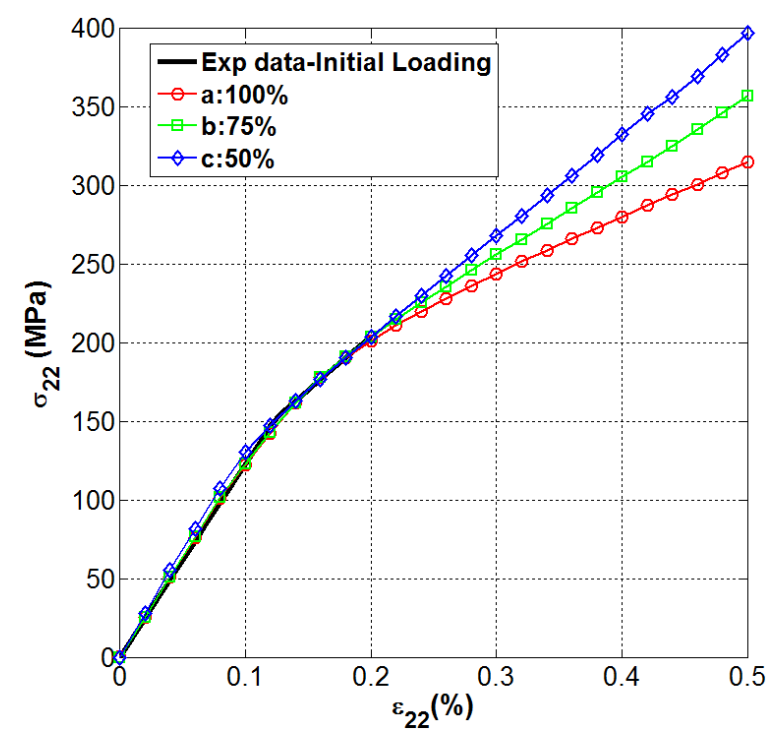

Figure 5.14 Optimized homogenized responses for three types of interface distributions.

\begin{tabular}{|l|c|c|c|c|c|}
\hline & Case a: $100 \%$ & Case b: $75 \%$ & \multicolumn{2}{c|}{ Case c: $50 \%$} \\
\hline$\sigma_{22}$ & \multicolumn{3}{|c|}{ Step 10:0.2\% } & \\
\hline \\
\hline$\sigma_{33}$
\end{tabular}

Figure 5.15 Full-field stress distributions at step 10 for case (a), (b) and (c). 


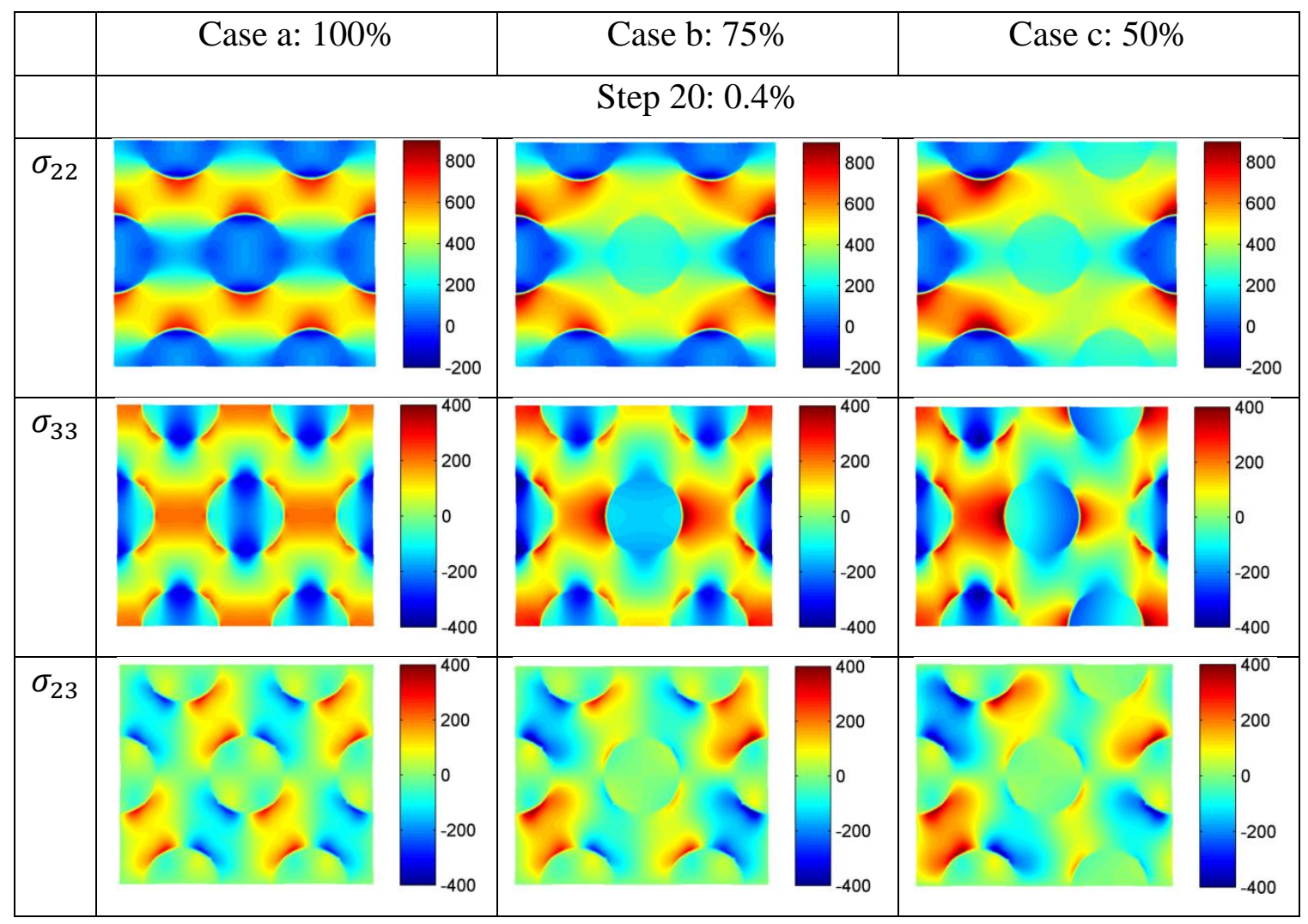

Figure 5.16 Full-field stress distributions at step 20 for cases (a), (b) and (c).

\subsection{Summary}

The feasibility of using the CZM-FVDAM theory as a driver for the PSO algorithm in search of optimum interfacial stiffness and strength parameters was first confirmed by incorporating the finite-deformation version of FVDAM (Hamed and Pindera, 2012) without damage evolution capability into the PSO algorithm in order to identify the optimal unit cell architectures and fiber and matrix material properties that targeted the response of three types of heart-valve chordae tendineae. This feasibility study illustrated that PSO is a well-suited technique for optimization problems involving objective functions that vary non-smoothly with design variable changes, and that the combination of homogenized-based model and PSO approach is a very powerful tool to quickly identify optimal design variables for the unit cell. Subsequently, the CZM-based FVDAM was incorporated into the PSO algorithm to identify interfacial parameters for the $\mathrm{SiC} / \mathrm{Ti}$ material system. Through optimization, interfacial parameters were efficiently identified without extensive parametric studies for different types of unit cell, such as square and hexagonal unit cells. Moreover, the effect of the number of active interfaces in a multi-inclusion 
unit cell on the homogenized response was identified, and explained by examining the underpinning stress transfer mechanisms affected by the absence of interfaces undergoing damage.

In the early interfacial damage stage for which experimental data was available, different combinations of interface distributions and interfacial parameters were shown to produce similar responses which correlated very well with experimental data. As expected, unit cells with fewer active fiber/matrix interfaces possess stronger loading capability with evolving damage. Hence the range over which the objective function is minimized for a given set of design variables plays an important role, and may produce different and non-optimal results outside this range.

Finally, interface distributions affect stress symmetry. For instance if the interface distribution is not symmetric, the stress distributions would also not possess symmetric characteristics, leading to potential loss of orthotropy under multiaxial loading for small unit cell sizes. Such important and fundamental issues as the effect of evolving damage on the preservation or loss of material orthotropy, and the related issue of the appropriate unit cell size used in the simulations, may efficiently be addressed using the developed CZM-based FVDAM theory in future studies. 


\section{Chapter 6}

\section{Damage Evolution in Cross-Ply Laminates}

\section{Revisited}

\subsection{Introduction}

In this chapter, we employ the FVDAM theory with the incorporated cohesive zone model to revisit the classical problem of damage evolution in symmetric cross-ply laminates under unidirectional loading due to progressive cracking of the transverse plies which may lead to delamination between the outer and inner plies when the transverse cracks reach saturation density, Schoeppner and Pagano (1999), Dharani et al. (2003). This hypothesis has been accepted by a number of researchers, but universal acceptance remains lacking, McCartney (2013). The above well-investigated problem serves as a model problem against which new damage evolution approaches may be gauged. It also provides a foundation for understanding damage evolution in symmetric laminates other than composites, such as those found in the microelectronics industry.

Hence the objectives of this investigation are several-fold. One objective is to demonstrate the finite-volume theory's ability to track the evolution of dispersed damage caused by the combined effects of transverse cracking and interfacial delamination, while accounting for the important effects of transverse ply strength variability and fabrication cooldown. Successful demonstration of this capability is intimately related to establishing the relationship between the homogenized response and the underpinning damage mechanisms, an ultimate goal of this investigation. In contrast with still-life approaches widely employed to assess the impact of damage on homogenized moduli and damage mode transition, the damage evolution in our approach is monitored on the fly within a unified homogenization framework that admits multidirectional loading. While it is known that certain homogenized moduli are substantially more sensitive to damage than others based on snapshot analyses of cracked laminates at fixed crack densities, herein we demonstrate on the fly during continuing loading that this sensitivity 
has dramatic impact on the homogenized Poisson's response features. This observation suggests a diagnostic tool for damage initiation detection and correlation with underpinning local failure mechanisms. We start with a brief historical survey of the literature to place our contribution and simulation approach in perspective.

\subsection{A Brief Historical Perspective}

The occurrence of successive cracks in the $90^{\circ}$ plies of symmetric cross-ply or $\left[0_{m} / 90_{n}\right]_{s}$ laminates loaded axially has been documented as early as the mid 1960's, see Vasilev et al. (1970) and the references therein. An approximate analytical model constructed by these authors based on the variational approach revealed that a system of cracks a distance $\pi / k_{2}$ apart develops in the $90^{\circ}$ layers when the ply strength is initially reached, where $k_{2}$ depends on the thickness and material properties of the $0^{\circ}$ and $90^{\circ}$ plies (see the Appendix I). Subsequent loading produces additional cracks in the $90^{\circ}$ plies halfway between the first set when the axial stress in these locations again reaches the ply strength. The resulting homogenized axial stress-strain curve is characterized by jogs that correspond to the successive occurrence of transverse cracks in the $90^{\circ}$ plies until sufficient stress is reached in the $0^{\circ}$ plies to cause catastrophic failure. The severity of these jogs that correspond to the energy released by the catastrophic fracture of $90^{\circ}$ plies depends on the relative proportion of the two sets of plies, and hence the energy associated with the stresses carried by the $90^{\circ}$ plies. This form of damage also degrades the homogenized moduli with the ensuing implications relative to structural analysis and integrity, and has been an intense area of research since then.

The above phenomenon has been re-discovered by Garrett and Bailey (1977), Parvizi and Bailey (1978), Bailey et al. (1979), Highsmith and Reifsnider (1982), and others. The period that followed was characterized by the development of numerous analytical and numerical models aimed at predicting moduli degradation of cross-ply and related symmetric laminates as a function of crack density, (Wang 1984, Wang et al. 1985, Talreja 1985, Groves et al. 1987, Gudmundson and Ostlund 1992, Lee and Hong 1993, Berthelot et al. 1996, Joffe and Varna 1999). The various models were based on shear lag assumptions of varying Joffe complexity, approximate elasticity-based analytical and variational approaches, and finite-element solutions. The variational approach employed by Vasilev et al. (1970) was reproduced by Hashin (1985, 1986), and applied to calculate homogenized moduli of symmetric cross-ply laminates with 
uniform crack spacing. It has been used extensively as a benchmark for comparison with other solutions as well as experimental data. More recently, boundary-element and finite-element solutions of unit cells representative of symmetric cross-ply laminates with evenly spaced transverse cracks have been reported, Paris et al. (2010a, b), Akula and Garnich (2012), as well as exact elasticity solutions based on the singular integral approach, Wang and Han (2010), and non-singular series expansion, Huang et al. (2011).

While the calculation of stiffness degradation due to transverse cracking in this class of laminates is well-established, the evolution of damage continues to receive considerable attention, Barbero and Cosso (2014). The problem is complicated by the occurrence of interfacial delaminations between the outer $0^{\circ}$ and inner $90^{\circ}$ plies which may arise when the transverse crack density reaches a saturation point, attributed to the occurrence of compressive normal stress between two adjacent transverse cracks that develops with sufficient crack density, Schoeppner and Pagano (1999). This compressive stress may be used as a criterion to determine at what point along the loading history the damage mode switches from transverse cracking to interfacial delaminations in order to calculate the effect of the combined damage on stiffness degradation, Dharani et al. (2003). While the majority of simplified analytical and finite-element unit cell models are based on uniform crack spacing, the in-situ $90^{\circ}$ ply strength has a statistical distribution which may affect crack density evolution in the early stages, Berthelot and Le Corre $(1999,2000)$. The role of the fabrication-induced residual stress in the crack density evolution is also important both at the ply and fiber/matrix level, with the latter not thoroughly explored. A thorough review of the literature on this topic up to early 2000's has been provided by Berthelot (2003). The current focus is the understanding of factors that affect the transition from vertical cracking of the $90^{\circ}$ plies to delamination of the $0^{\circ} / 90^{\circ}$ interface, Hoiseth and Qu (2003), Paris et al. (2010a, b), as will be discussed in the sequel.

The mentioned approaches for the above class of laminates have been mostly employed in a stand-alone fashion to estimate the effect of crack density on stiffness degradation and to understand the effect of damage modes on the related stress fields which drive subsequent damage evolution. While it is possible to use these approaches to reconstruct the overall or homogenized stress-strain response of symmetric cross-ply laminates under simple uniaxial loading, relatively little work has been done in constructing a homogenization theory leading to the homogenized Hooke's law valid under combined three-dimensional loading which takes into 
account the various damage modes, their evolution and transition. Such capability is important in structural analyses of laminated composites where damage evolution contributes substantially to the overall nonlinear response. The FVDAM theory with cohesive zone model capability briefly described below provides these means. An alternative approach to damage evolution based on the original FVDAM theory has been proposed by Tang et al. (2015). The use of cohesive zone models to naturally track the evolution of damage in cross-ply laminates is also being pursued within the finite-element framework, Shi et al. (2014), albeit without (thus far) accounting for the possibility of interfacial delamination.

\subsection{Material Systems Used in Numerical-Experimental Correlation}

We employ two sets of experimental data on the tensile response of symmetric graphite/epoxy cross-ply laminates to gauge the FVDAM theory's capability in capturing the homogenized response (both axial and transverse), crack density evolution, damage mode bifurcation and the underpinning mechanisms. The first data set had been generated using $\left[0 \frac{0}{2} / 90_{2}^{0}\right]_{s}$ laminates fabricated with unidirectionally-reinforced AS4/3501 graphite/epoxy prepreg containing 0.63 fiber volume fraction, Pindera (1986). The average ply thickness of the fabricated specimens comprised of eight layers was $0.1315 \mathrm{~mm}$. For this set, laminate axial tensile and transverse Poisson's responses are available, but no information on the crack density as a function of applied axial load. The $\left[0 \frac{0}{2} / 90_{2}^{0}\right]_{s}$ graphite/epoxy laminate specimens were tested to failure under monotonic loading normal to the inner $90^{\circ}$ plies. Axial and transverse strains were recorded using 0.125 in $(3.175 \mathrm{~mm})$ strain gages bonded to the outer $0^{\circ}$ plies which span almost two thirds of the unit cell length used in the simulations.

Unidirectional on-axis and off-axis specimens fabricated using the same prepreg and consolidation process were also tested under tensile loading to determine in-plane elastic moduli needed to simulate homogenized laminate response and ply-level stress fields. The tension tests yielded four of the five elastic moduli necessary for simulating the laminate response. The fifth elastic modulus and thermal expansion coefficients needed to simulate post-consolidation cooldown and mechanical loading were obtained from FVDAM analysis based on hexagonal unit cell representation of the tested unidirectional graphite/epoxy composite shown in Figure 6.1a. The fiber and matrix elastic moduli employed in the micromechanical analysis are given in Table 6.1, and the complete set of thermo-elastic ply-level moduli is reported in Table 6.2. Lastly, 
the average axial and transverse strengths obtained from the tested $0^{\circ}$ and $90^{\circ}$ specimens were 1,550 and 51.7 MPa, respectively.

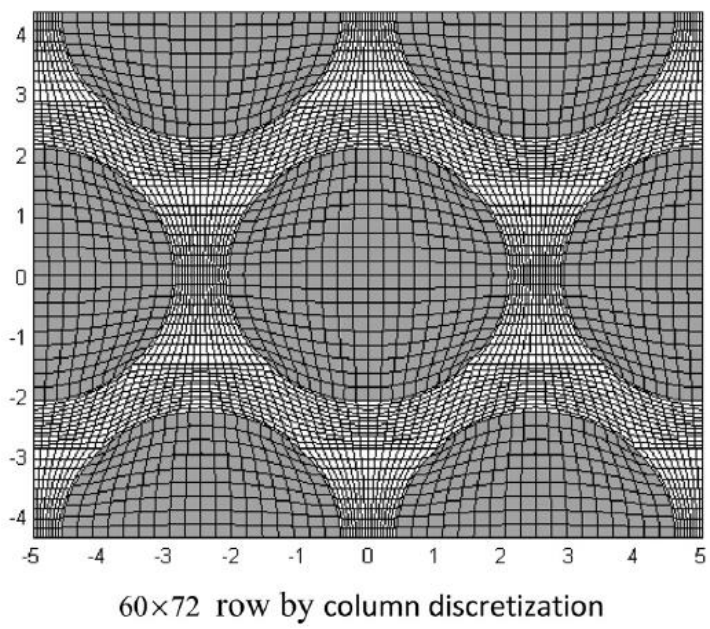

(a)

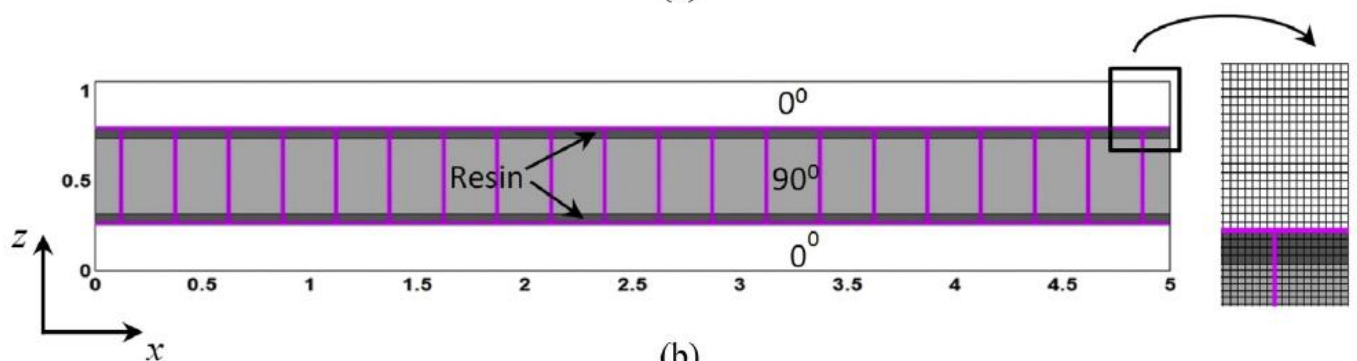

(b)

Figure 6.1 (a) Hexagonal unit cell of unidirectional AS4/3501 graphite/epoxy with fiber volume fraction of 0.63 used to generate the missing thermo-elastic moduli for laminate analysis; (b) unit cell of a $\left[02 / 90_{2}^{0}\right]_{s}$ laminate.

The second data set on similar symmetric cross-ply graphite/epoxy laminates with individual ply thickness of $0.132 \mathrm{~mm}$ and thermo-elastic ply-level moduli given in Table 6.3 had been generated by Wang et al. (1985), and was chosen to complement the first set. For this set, crack density evolution as a function of applied load is available for laminates with different number of the $90^{\circ}$ plies. We employ the reported crack density evolution data for $\left[0 \frac{0}{2} / 90^{\circ}\right]_{S}$ and $\left[0_{2}^{0} / 90_{2}^{0}\right]_{s}$ laminates to further validate the predictive capability of the FVDAM theory.

Table 6.1 Thermoelastic properties of the AS4 graphite fiber and 3501 epoxy matrix. Note that the AS4 fiber is assumed to be transversely isotropic with $E_{22}=E_{33}, v_{12}=v_{13}$ and $G_{23}=$ $E_{22} / 2\left(1+v_{23}\right)$ and the epoxy matrix is isotropic (Soden et al. 1998).

\begin{tabular}{|c|c|c|c|c|c|c|c|}
\hline Material & $E_{11}(\mathrm{GPa})$ & $E_{22}(\mathrm{GPa})$ & $G_{12}(\mathrm{GPa})$ & $G_{23}(\mathrm{GPa})$ & $v_{12}$ & $\alpha_{11}\left(\mu /{ }^{\circ} \mathrm{C}\right)$ & $\alpha_{22}\left(\mu /{ }^{\circ} \mathrm{C}\right)$ \\
\hline AS4 fiber & 225 & 15 & 15 & 7 & 0.2 & -0.5 & 15 \\
\hline 3501 epoxy & 4.2 & 4.2 & 1.57 & 1.57 & 0.34 & 45 & 45 \\
\hline
\end{tabular}


Table 6.2 Thermoelastic properties of the unidirectional AS4/3501 graphite/epoxy laminate with the fiber volume fraction of 0.63 , measured experimentally in the case of the moduli $E_{11}, E_{22}$ and $G_{12}$ and $v_{12}$ and in the case of $G_{23}, \alpha_{11}$ and $\alpha_{22}$ calculated using the homogenization FVDAM theory.

\begin{tabular}{|c|c|c|l|c|c|c|}
\hline$E_{11}(\mathrm{GPa})$ & $E_{22}(\mathrm{GPa})$ & $G_{12}(\mathrm{GPa})$ & $G_{23}(\mathrm{GPa})$ & $v_{12}$ & $\alpha_{11}\left(\mu /{ }^{\circ} \mathrm{C}\right)$ & $\alpha_{22}\left(\mu /{ }^{\circ} \mathrm{C}\right)$ \\
\hline 139.3 & 9.65 & 4.48 & 3.56 & 0.28 & 0.047 & 30.6 \\
\hline
\end{tabular}

Table 6.3 Thermoelastic properties of the unidirectional graphite/epoxy laminate tested by Wang et al. (1985).

\begin{tabular}{|c|c|c|c|c|c|c|}
\hline$E_{11}(\mathrm{GPa})$ & $E_{22}(\mathrm{GPa})$ & $G_{12}(\mathrm{GPa})$ & $G_{23}(\mathrm{GPa})$ & $v_{12}$ & $\alpha_{11}\left(\mu /{ }^{\circ} \mathrm{C}\right)$ & $\alpha_{22}\left(\mu /{ }^{\circ} \mathrm{C}\right)$ \\
\hline 144.8 & 11.7 & 6.5 & 3.8 & 0.3 & 0.36 & 28.8 \\
\hline
\end{tabular}

\subsection{Damage Modes and Enabling Stress Fields}

In order to understand the evolution of damage modes in symmetric cross-ply laminates we first analyze stress fields as a function of transverse crack density, both without and with resin-rich region at the $0^{\circ} / 90^{\circ}$ ply interface and horizontal delamination of different lengths, using the first set of data. The approach is to take snapshots of the laminate at different crack densities to understand how damage may subsequently evolve. In particular, we address the proposed hypothesis of damage mode bifurcation from transverse cracking to interfacial delamination upon transverse crack density saturation. In this initial analysis, cracks are treated as traction-free surfaces with the interfacial traction-displacement separation law on the left side of Eq. (2.21) replaced by zero. The unit cells of the $\left[0_{2}^{0} / 90_{2}^{0}\right]_{s}$ laminate employed in the still-life analysis contained one vertical crack in the center with the crack density controlled by adjusting the unit cell's length.

At first, transverse cracks in the $90^{\circ}$ plies occur far apart with negligible interaction. Figure 6.2 illustrates stress distributions within $90^{\circ}$ and $0^{\circ}$ plies in the vicinity of an isolated transverse crack as a function of normalized distance $\mathrm{x} / \mathrm{t}$ from the crack front at different elevations $z / t$ from the midplane, where $2 t$ is the total thickness of the inner $90^{\circ}$ plies, including the interfacial resin-rich layer. The stress distributions have been normalized by the axial stress $\sigma_{x x}^{o}$ that would develop in undamaged $90^{\circ}$ plies. The crack density corresponding to this damage state is 0.19 cracks $/ \mathrm{mm}$, yielding crack spacing ten times the inner ply thickness, or 20t. The axial stress $\sigma_{x x}$ in the $90^{\circ}$ plies varies pronouncedly in the thickness direction, in contrast with 
the variational solution of Vasilev et al. (1970) which neglects this variation, and asymptotically attains its uncracked value for all elevations approximately two and a half crack lengths from the crack front. Hence another set of cracks will occur at that distance in the absence of transverse strength variation. The local maxima very close to the vertical crack face in the $90^{\circ}$ plies along the $0^{\circ} / 90^{\circ}$ ply interface suggests a potential crack initiation site. Complete through-thickness propagation requires sufficient crack-driving force which, however, may not be available due to the crack-shielding effect.

The vertical crack produces singular-like behavior in the transverse shear and normal stress components $\sigma_{x z}$ and $\sigma_{z z}$, respectively, along the $0^{\circ} / 90^{\circ}$ ply interface. While the normal stress $\sigma_{z z}$ is compressive in the middle of the $90^{\circ}$ ply immediately adjacent to the crack face, it becomes tensile at the $0^{\circ} / 90^{\circ}$ ply interface, serving as a potential delamination driver. The variational solution fails to predict this stress reversal, making it unsuitable for delamination analysis, but provides a reasonable estimate in the midplane. Interfacial shear stress at the crack tip of comparable magnitude provides additional delamination driving energy. The vertical crack also alters the stress field in the $0^{\circ}$ plies, producing substantial amplification in the axial stress $\sigma_{x x}$ directly ahead of the crack front that mimics the singular elasticity solution for a crack perpendicular to a bi-material interface. This stress amplification may produce local fiber breakage in the final stage of the damage evolution process, leading to the $0^{\circ}$ ply fracture and hence laminate failure.

Increasing crack density alters the stress fields in the $90^{\circ}$ and $0^{\circ}$ plies as shown in Figure 6.3 for the crack density of 0.95 cracks $/ \mathrm{mm}$ or crack spacing of $4 \mathrm{t}$. In contrast with the isolated crack case, the axial stress $\sigma_{x x}$ attains different asymptotic values at different elevations halfway between adjacent cracks, indicating that the propensity for the formation of new cracks decreases. A local maximum in the axial stress is also observed along the $0^{\circ} / 90^{\circ}$ ply interface close to the crack face, suggesting a potential crack initiation site (but not an instantaneous vertical crack formation). The transverse normal and shear stresses $\sigma_{z z}$ and $\sigma_{x z}$ at the $0 \% / 90^{\circ}$ interface remain little affected by the crack spacing decrease from $20 \mathrm{t}$ to $4 \mathrm{t}$, suggesting little change in delamination initiation propensity. The axial stress concentration in the $0^{\circ}$ ply directly above the vertical crack also remains unchanged, promoting local fiber breakage with increasing load.

Figure 6.4 summarizes the results in the above two figures for the crack densities 0.19 , $0.95,1.95$ and $3.80 \mathrm{cracks} / \mathrm{mm}$ or crack spacings of $20 \mathrm{t}, 4 \mathrm{t}, 2 \mathrm{t}$ and $\mathrm{t}$, respectively. The through- 
thickness variation of the normal stress $\sigma_{x x}$ halfway between two adjacent vertical cracks is presented as a function of the normalized coordinate $z / t$ whereas the transverse shear and normal stresses $\sigma_{x z}$ and $\sigma_{z z}$ are given along the $0^{\circ} / 90^{\circ}$ interface as a function of the normalized axial coordinate $x / t$. These distributions demonstrate the interplay between decreasing axial stress in the $90^{\circ}$ plies with increasing crack density, and interfacial tractions along the $0^{\circ} / 90^{\circ}$ interface potentially leading to delamination. As observed and discussed by others, Schoeppner and Pagano (1999), Dharani et al. (2003), the axial stress halfway between two adjacent vertical cracks decreases to a negative value over a substantial distance separating the $0^{\circ}$ plies with large enough crack density. This partially supports the hypothesis regarding vertical crack density saturation and subsequent delamination initiation, or damage mode bifurcation. On the other hand, crack-tip concentrations in the interfacial normal and transverse shear stresses are attenuated with increasing crack density, suggesting decreasing propensity for delamination as the vertical crack spacing reaches saturation. To address this, the effect of local delamination emanating from the vertical crack tip is considered below. The effects of resin-rich layer at the $0^{\circ} / 90^{\circ}$ interface on stress fields between interacting vertical cracks within $90^{\circ}$ plies as a function of crack density are included in Figure 6.4. The presence of a softer thin layer of matrix separating the $0^{\circ}$ and $90^{\circ}$ plies produces further reductions in the through-thickness and interfacial stress distributions, accelerating crack density saturation.

The effect of interfacial debonding between the resin-rich layer and the $0^{\circ}$ ply on stress fields between interacting vertical cracks within $90^{\circ}$ plies is shown in Figure 6.4 for the four crack densities and two initial delamination lengths of $0.2 \mathrm{t}$ and $0.4 \mathrm{t}$. While the through-thickness axial stress halfway between two adjacent vertical cracks is little affected by the delaminations, the crack-tip magnitudes of the interfacial normal and transverse shear stress experience substantial changes. For a fixed vertical crack density, the maximum normal stress at the delamination tip decreases with increasing delamination length relative to the intact interface. In contrast, the maximum transverse shear stress increases with increasing delamination, potentially providing sufficient driving force. 

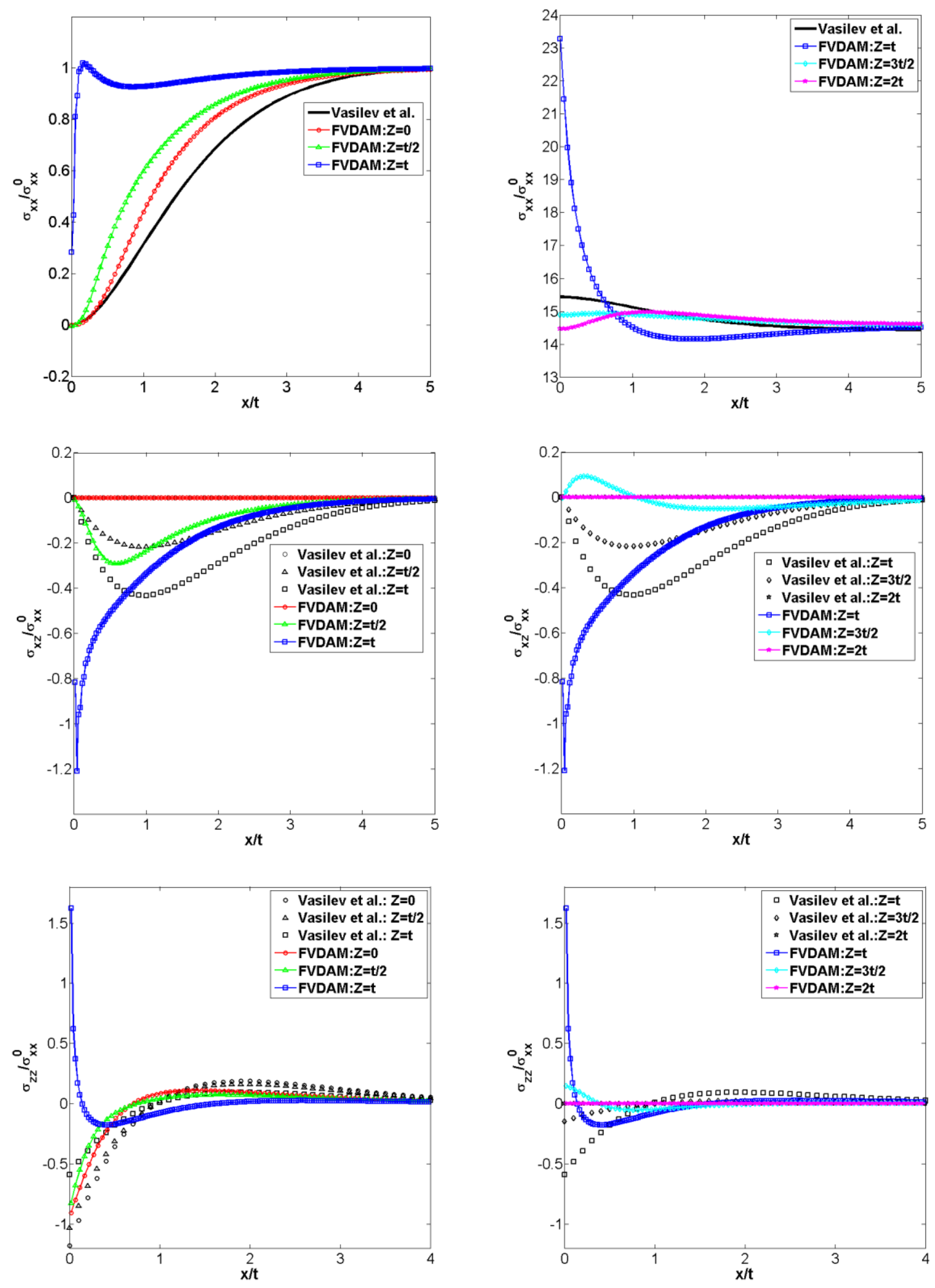

(a) $90^{\circ}$ plies

(b) $0^{\circ}$ plies

Figure 6.2 Stress distributions in $90^{\circ}$ and $0^{\circ}$ plies of a graphite/epoxy $\left[0 \frac{0}{2} / 90_{2}^{0}\right]_{\mathrm{s}}$ laminate in the vicinity of an isolated vertical crack (crack density of 0.19 cracks $/ \mathrm{mm}$ ) at different elevations from the midplane. Comparison with the variational solution of Vasilev et al. (1970). 

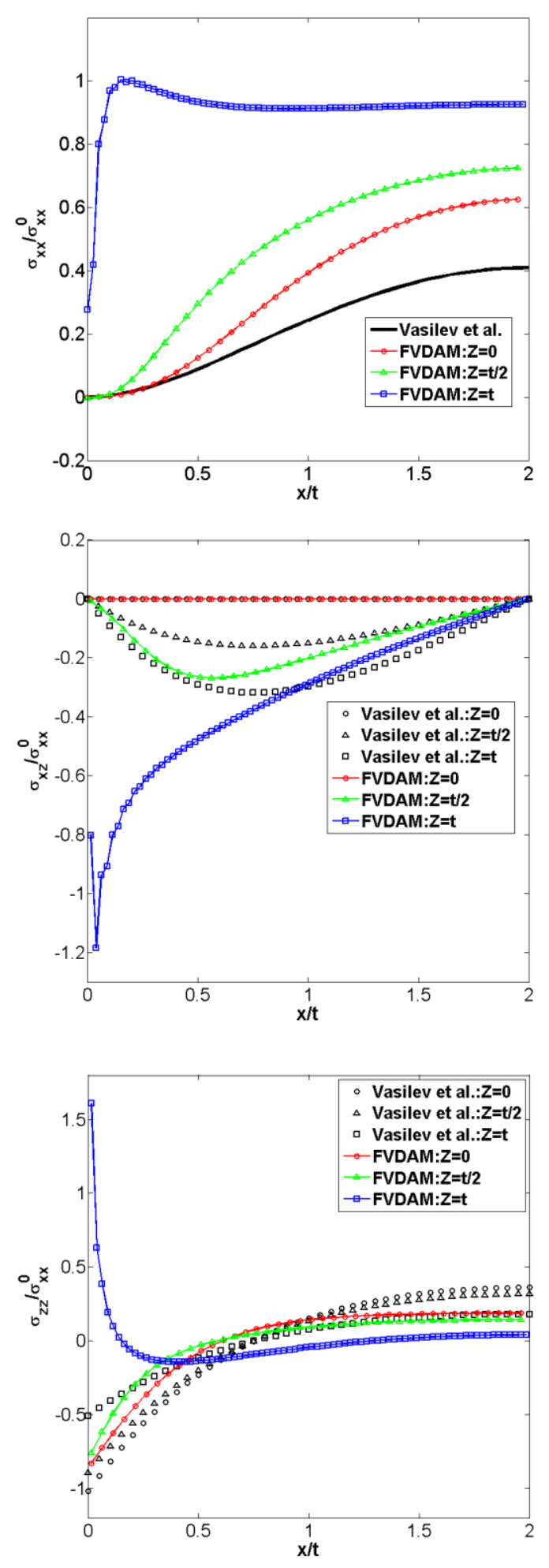

(a) $90^{\circ}$ plies
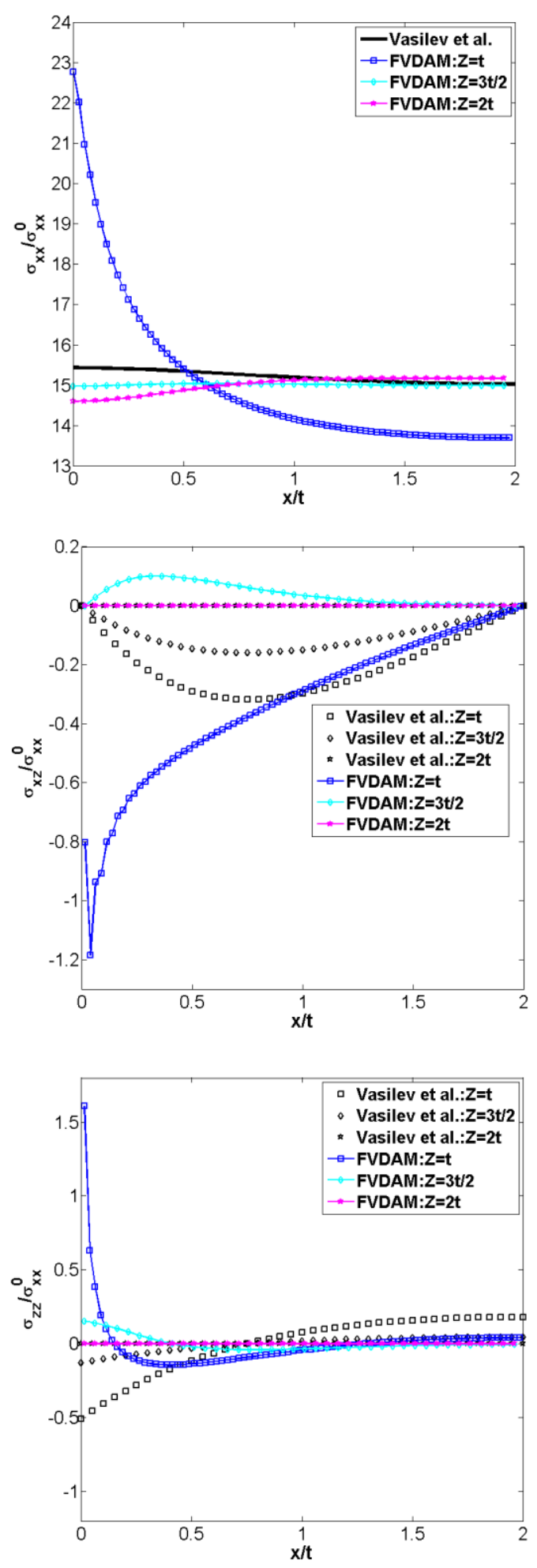

(b) $0^{\circ}$ plies

Figure 6.3 Stress distributions in $90^{\circ}$ and $0^{\circ}$ plies of a graphite/epoxy $\left[0 \frac{0}{2} / 90_{2}^{0}\right]_{\mathrm{s}}$ laminate between interacting vertical cracks (crack density of 0.95 cracks $/ \mathrm{mm}$ ) at different elevations from the midplane. Comparison with the variational solution of Vasilev et al. (1970). 


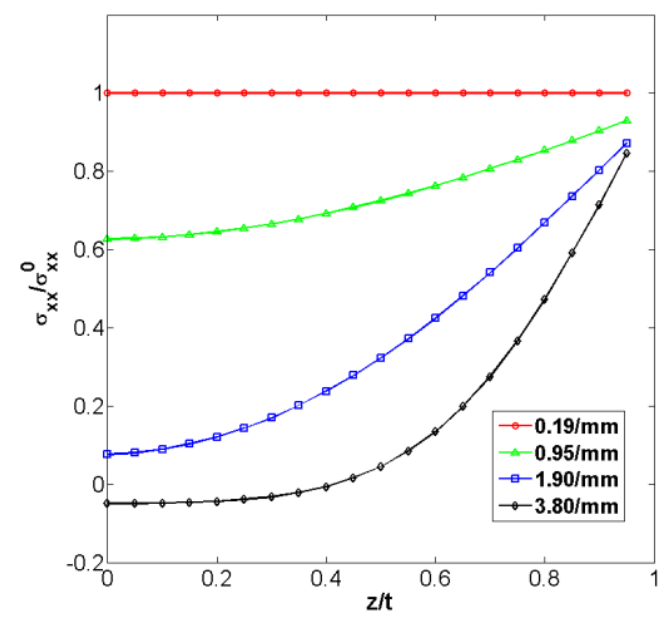

(a)
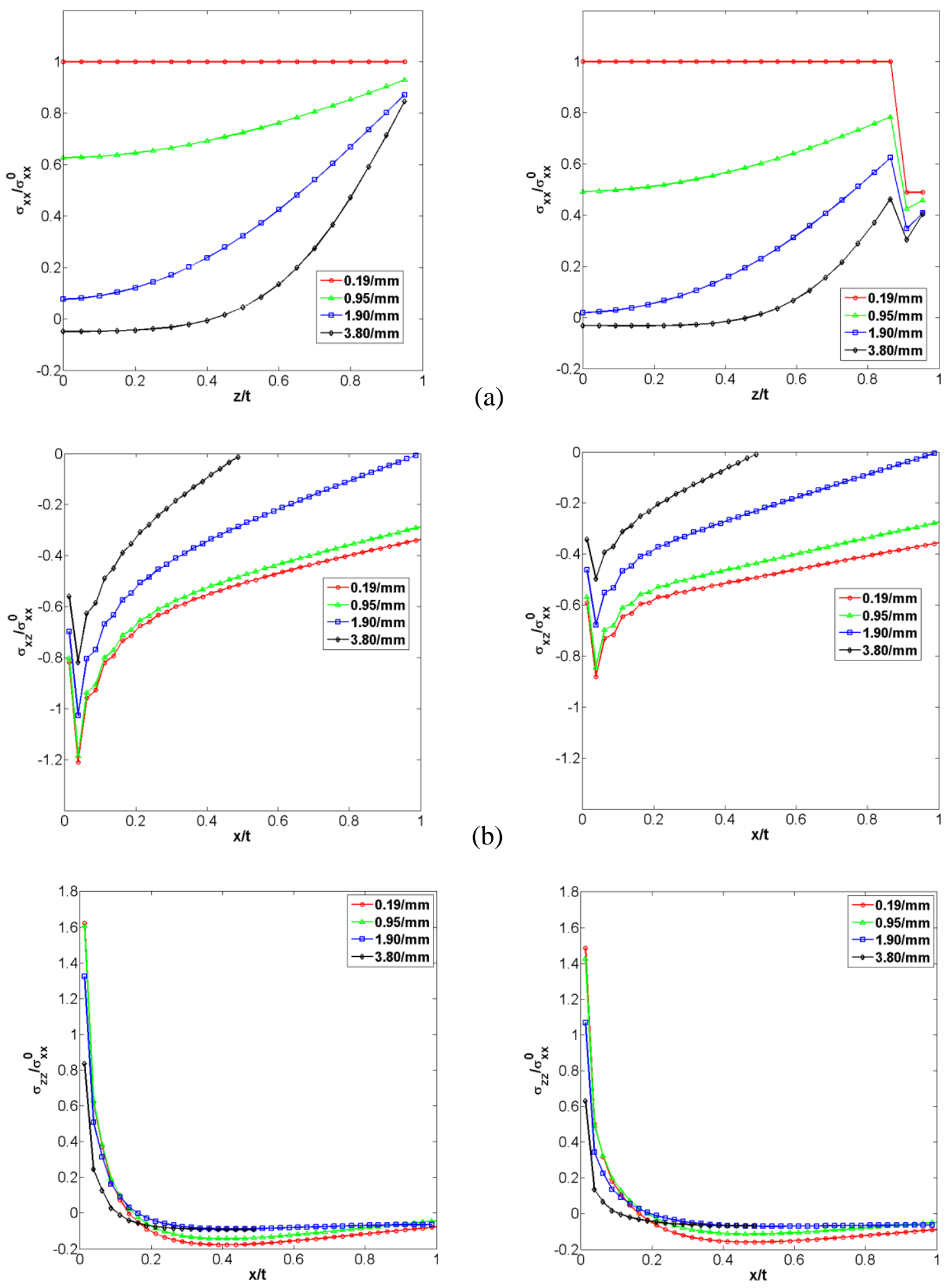

without resin-rich layer

(c)

with resin-rich layer

Figure 6.4 Effect of resin-rich layer and crack density on stress distributions between adjacent vertical cracks in $90^{\circ}$ plies of a $\left[00_{2}^{0} / 90_{2}^{0}\right]_{s}$ laminate: (a) through-thickness $\sigma_{x x}$ stress distributions halfway between vertical cracks; (b-c) $\sigma_{x z}$ and $\sigma_{z z}$ stress distributions along the $0^{\circ} / 90^{\circ}$ interface. 


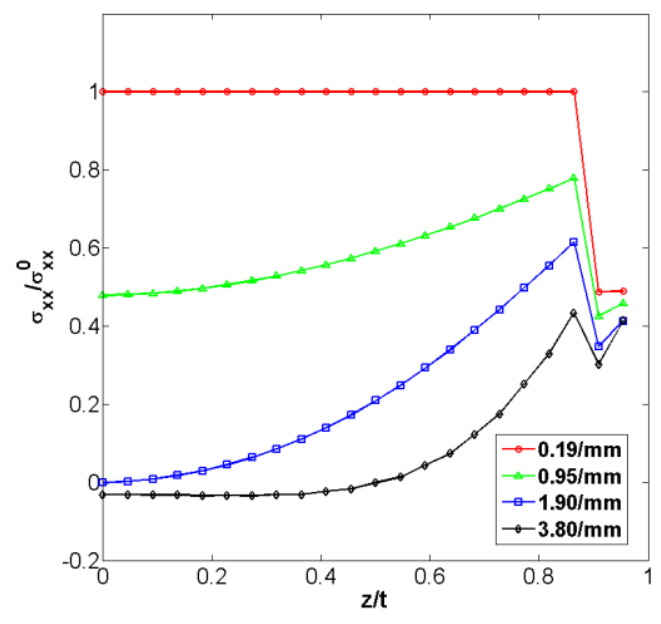

(a)
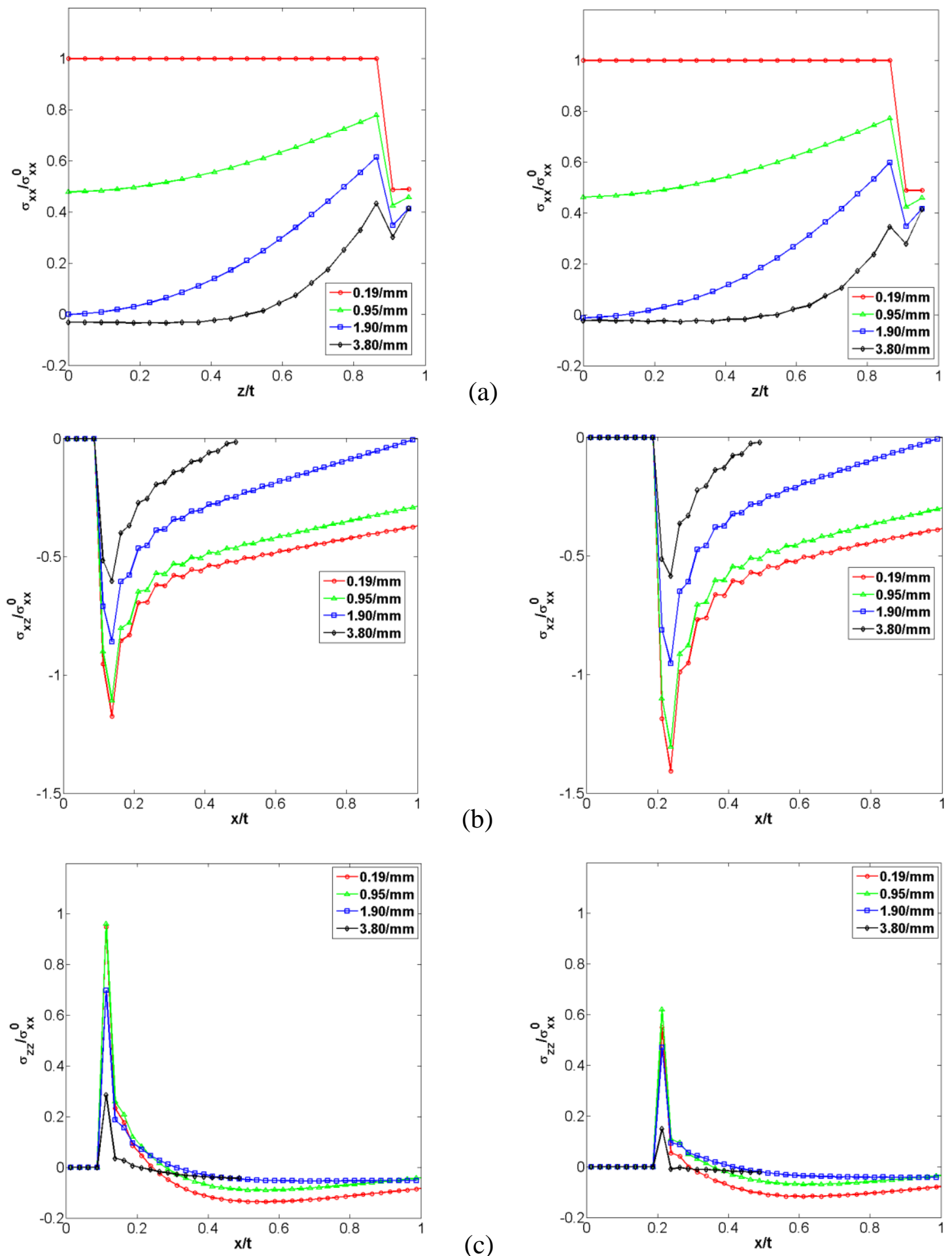

(b)

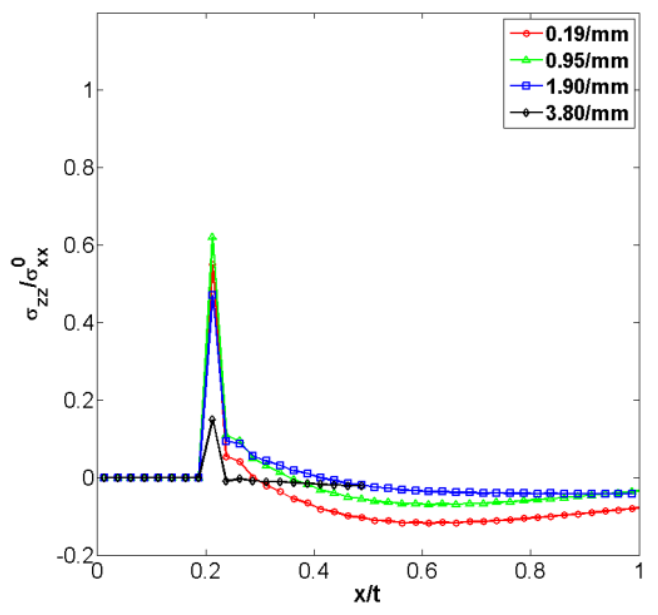

(a) $0.2 \mathrm{t}$ delamination length

(b) $0.4 \mathrm{t}$ delamination length

Figure 6.5 Effect of interfacial debonding between the resin-rich layer at the $0^{\circ} / 90^{\circ}$ interface and the $0^{\circ}$ ply on stress distributions between adjacent vertical cracks in $90^{\circ}$ plies of a $\left[0_{2}^{\circ} / 90_{2}^{\circ}\right]_{s}$ laminate: (a) through-thickness $\sigma_{x x}$ stress distributions halfway between vertical cracks; (b-c) $\sigma_{x z}$ and $\sigma_{z z}$ stress distributions along the $0^{\circ} / 90^{\circ}$ interface. 


\subsection{Homogenized Axial and Transverse Response: Theory vs Experiment}

Given the preceding still-life analysis of the damage modes and their interaction, we conduct damage evolution simulations where vertical cracks and $0^{\circ} / 90^{\circ}$ delaminations are allowed to occur on the fly, consistent with the cohesive-zone model constraint, as the cross-ply laminate is loaded. In simulating the laminate response in the presence of evolving damage, we account for the fabrication-induced residual stresses by first subjecting the laminate to a temperature cooldown of $\Delta \mathrm{T}=-100^{\circ} \mathrm{C}$ followed by mechanical loading due to uniaxial stress $\bar{\sigma}_{x x}$.

The unit cell of the $\left[00_{2}^{0} / 90_{2}^{0}\right]_{s}$ laminate with homogenized ply moduli and dimensions used in the damage evolution simulations is shown in Figure 6.1b. The axial dimension of the unit cell, $5 \mathrm{~mm}$, is sufficiently long to accommodate large enough number of vertical interfaces to enable modeling of the laminate response with nonuniform strength of the $90^{\circ}$ plies. Towards this end, twenty equally-spaced vertical interfaces that mimic the fracture process using the cohesive-zone model were inserted along the length of the unit cell. Hence the maximum crack density that can be obtained with this unit cell is 4 cracks $/ \mathrm{mm}$ if all the interfaces separate to produce vertical cracks, which is substantially greater than experimentally observed for this cross-ply laminate. Each vertical interface was assigned a different strength using random distribution in the range [77 87] MPa. Included in the unit cell model is a thin resin-rich layer between the $90^{\circ}$ and $0^{\circ}$ plies that was observed after fabrication. Horizontal interfaces were also inserted along the $0^{\circ} / 90^{\circ}$ ply interface in order to simulate potential interfacial degradation and delamination. The cohesive zone model parameters for the vertical and horizontal interfaces are given in Table 6.4. As the vertical crack growth tends to proceed catastrophically at first, (Wang 1984), characterized by distinct jogs in the homogenized response and hence a sudden release of energy, no degradation of the vertical interfaces was assumed past the maximum stress in the cohesive zone model. The discretization of the above unit cell into $88 \times 400$ rows and columns produced the same discretization per vertical interface/crack as the unit cells constructed for the still-life analyses.

The lower limit of the vertical interface strength distribution corresponds to the ply-level axial stress in the $90^{\circ}$ plies at the first-ply failure event observed in the laminate stress-strain response, which was calculated from the FVDAM simulation of temperature cool-down and 
initial mechanical loading. This range is higher than the average transverse strength of $51.7 \mathrm{MPa}$ of the tested $90^{\circ}$ specimens, and is consistent with the experimental observations of Bader et al. (1979) and the argument based on the availability of energy that drives the vertical crack, as also discussed by Wang (1984). The difference, which depends on the number of $90^{\circ}$ plies, is rooted in the constraining effect of the external $0^{\circ}$ plies and the available energy trapped within the $90^{\circ}$ plies. In addition to the impact at the ply-level, this constraint also alters the constituent-level stress field and hence the microstructure-controlled vertical crack propagation characteristics. The impact of this constraining effect on constituent-level stress fields is discussed below. The effect of $90^{\circ}$ ply microstructure on vertical crack growth characteristics will be addressed elsewhere.

\subsubsection{The Effect of Residual Stresses}

The fabrication process induces residual stresses at the fiber/matrix level within the ply and at the ply level within the laminate. Hence the in-situ strength of the $90^{\circ}$ plies in a cross-ply laminate may be different than that of the $90^{\circ}$ plies tested separately due to the constraint of the $0^{\circ}$ plies which alters the residual stress state. If the transverse failure is due to a combination of progressive debonding of the fiber/matrix interface, crack propagation through the fibers, and fracture of the surrounding matrix, then the fabrication-induced residual stresses will play a role. While this issue has been recognized in the literature, little work has been done to separate the influence of the constituent-level and ply-level residual stresses. Hence in this section we perform an analysis of the fabrication-induced residual stresses in $90^{\circ}$ plies separately as well as in-situ in the presence of the constraint of the outer $0^{\circ}$ plies, and compare the resulting stress fields at failure using the stand-alone $90^{\circ}$ specimen strength. The question that we address is whether the $90^{\circ}$ ply strength obtained from unidirectional specimens tested alone may be used directly in the cohesive zone model as a maximum interface strength parameter in simulating damage evolution with residual stresses accounted for at the ply-level.

To generate constituent-level stress fields in the unidirectional $90^{\circ}$ specimens at failure, temperature cooldown of $\Delta \mathrm{T}=-100^{\circ} \mathrm{C}$ was first applied followed by unidirectional loading by $\bar{\sigma}_{x x}$ up to the measured failure stress of $51.7 \mathrm{MPa}$. The stress fields were generated using the repeating unit cell with hexagonal array of graphite fibers in the epoxy matrix shown in Fig.6.3, representative of the unidirectional composite, with the thermoelastic moduli reported in Table 
6.1. The ply-level stress field history was then generated by subjecting the $\left[0 \frac{0}{2} / 90_{2}^{0}\right]_{s}$ laminate, with the homogenized elastic moduli obtained from the hexagonal unit cell analysis, to the same temperature change followed by uniaxial loading applied to the laminate up to the point at which the transverse stress in the $90^{\circ}$ plies reached the ply strength. This thermo-mechanical loading history was then employed in the calculation of constituent-level stress fields in the $90^{\circ}$ plies of the $\left[0_{2}^{0} / 90_{2}^{0}\right]_{s}$ laminate for comparison with the corresponding stress fields in unidirectional $90^{\circ}$ plies analyzed separately.

The constituent-level stress fields that develop in unidirectional $90^{\circ}$ specimens after fabrication cooldown and subsequent loading to failure are compared with the corresponding fields in the $90^{\circ}$ plies of the $\left[0_{2}^{0} / 90_{2}^{0}\right]_{S}$ laminate in Figure 6.6. The constraining effect of the $0^{\circ}$ plies produces strictly tensile $\sigma_{x x}$ stress field in the fiber and matrix phases of the in-situ $90^{\circ}$ plies immediately after the fabrication cooldown with maximum values occurring between adjacent fibers. This is in contrast with the unconstrained plies wherein compressive $\sigma_{x x}$ stress occurs in the same location. Subsequent mechanical loading further increases the tensile stress $\sigma_{x x}$ in the constrained $90^{\circ}$ plies between adjacent fibers to the value of $76.6 \mathrm{MPa}$ at the laminate stress that produces ply-level axial stress of 51.7 MPa corresponding to transverse strength of the tested unidirectional specimens. The above constituent-level stress is somewhat higher than the corresponding stress in the unconstrained plies, $68.5 \mathrm{MPa}$, due to the initially compressive cooldown stress. Nonetheless, the normal stress field in the constrained $90^{\circ}$ plies is not as conducive to rapid matrix or fiber/matrix fracture due to the observed horizontal bands of low stress regions through which a crack would need to propagate in order to cause complete failure. This is in contrast with the unconstrained $90^{\circ}$ plies where the normal stress between horizontal rows of fibers is substantially larger, promoting vertical crack growth through the matrix phase and around the fiber/matrix interface. The above observations suggest that the in-situ fracture strength of the $90^{\circ}$ plies is larger than the strength determined from uniaxial tensile tests on $90^{\circ}$ coupons. This is consistent with energy-release rate arguments employed to explain the typically larger in-situ $90^{\circ}$ ply strength and the experimental observation that this in-situ strength decreases with increasing $90^{\circ}$ ply thickness, (Crossman and Wang 1982, Wang 1984, and Wang et al. 1985). 

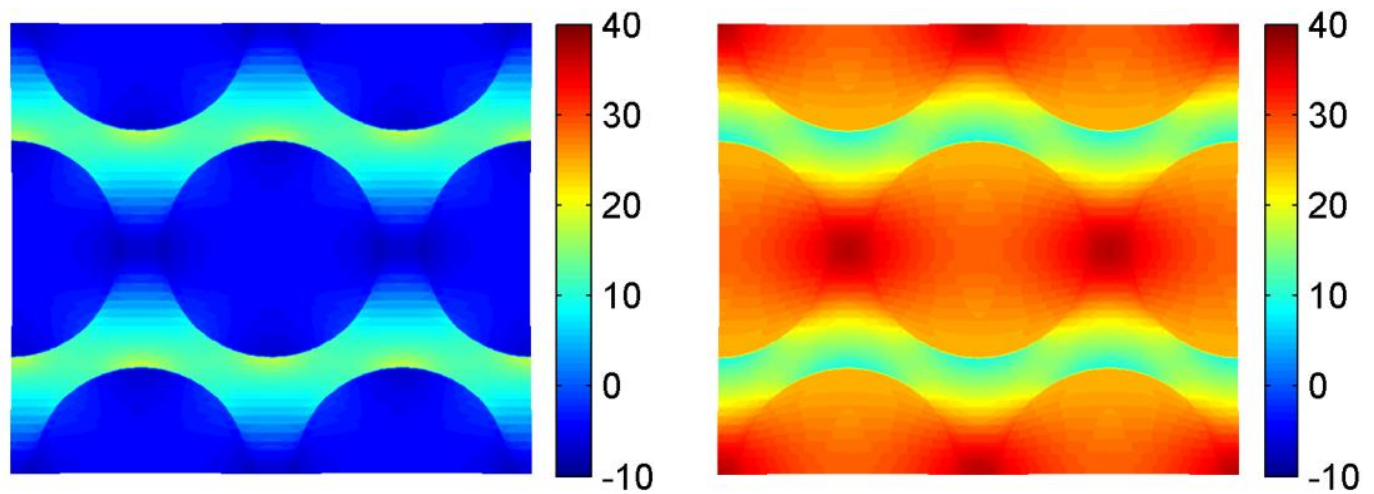

(a) After cooldown
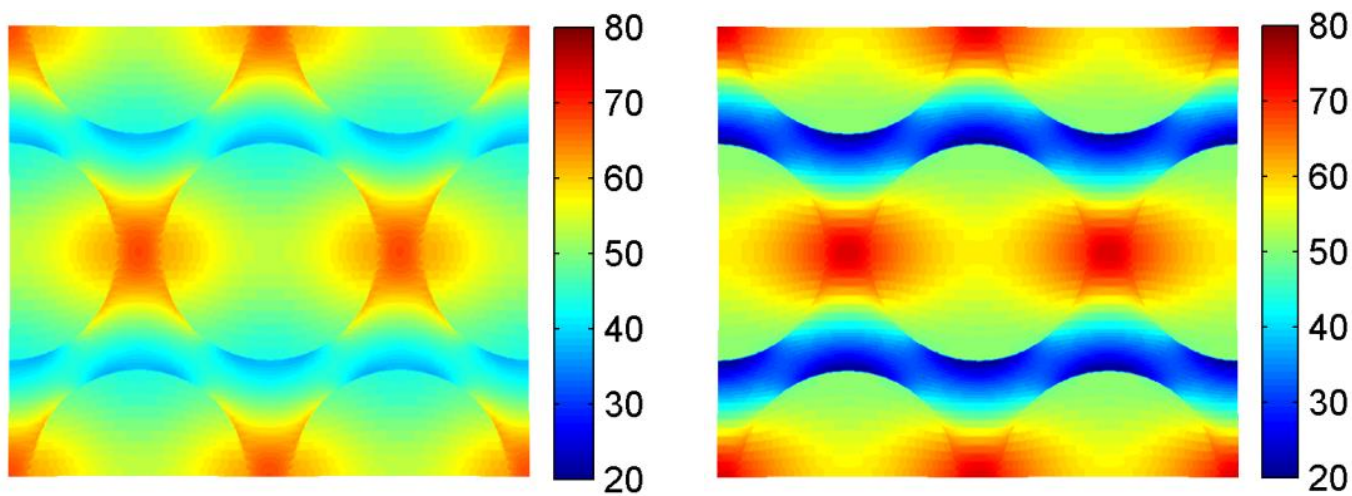

(b) After mechanical loading corresponding to initial failure of $51.7 \mathrm{MPa}$ in the $90^{\circ}$ plies.

Figure 6.6. Effect of fabrication cooldown on the microlevel $\sigma_{x x}$ stress fields in stand-alone (left) and in-situ (right) $90^{\circ}$ plies at failure.

\subsubsection{Features of the Homogenized Response and Underpinning Mechanisms}

The simulated homogenized axial stress-strain and transverse Poisson's responses are compared with the experimental data obtained from the tested graphite/epoxy $\left[0 \frac{0}{2} / 90_{2}^{0}\right]_{S}$ laminate in Figure 6.7a. Three laminate cases have been considered that illustrate the effects of resin-rich layer at the $0^{\circ} / 90^{\circ}$ interface, and uniform versus random vertical strength distributions for fixed cohesive zone parameters that characterize the horizontal interface strength, Table 6.4. These are laminates with variable and uniform transverse strength of the $90^{\circ}$ plies, respectively, each with resin-rich layer, and a $\left[0 \frac{0}{2} / 90_{2}^{0}\right]_{s}$ laminate with variable strength of the $90^{\circ}$ plies without resinrich layer. In the case of laminates with variable $90^{\circ}$ ply strength, representative responses of five realizations are shown in the figure. While the first-ply failure event is clearly discernible in the homogenized axial responses of all three laminates, subsequent fracture events are masked to a large extent. Moreover, little effect of the resin-rich layer region and vertical strength 
variability is observed. For the considered cross-ply laminates with equal proportion of $0^{\circ}$ and $90^{\circ}$ plies, the contribution of the $90^{\circ}$ plies towards axial load-bearing capacity is low due to the low Young's modulus in the direction of loading. Hence this is reflected in the insensitivity of the homogenized axial response to the varied parameters. This is in stark contrast with the transverse Poisson's response where evidence of multiple vertical fracture events is clearly observed throughout the entire response. Inclusion of resin-rich layer yields simulated homogenized Poisson's response closest to the experimental data in the linear region before the onset of progressive vertical fractures. The absence of the resin-rich layer lowers the transverse Poisson's response noticeably. The vertical fracture events manifest themselves as distinct jogs in the homogenized Poisson's response, closely mimicked by the FVDAM simulations. The jogs are smaller and more in line with the experimental results for simulations with the resin-rich layer and variable $90^{\circ}$ ply strength than the corresponding results based on uniform strength. The large jogs observed in the transverse Poisson's response based on uniform $90^{\circ}$ ply strength and resinrich layer presence indicate rapid formation of multiple vertical fractures with small load increases, which reduce the constraint on the transverse deformation of $0^{\circ}$ plies .

The FVDAM simulations for the considered three laminate cases are characterized by apparently straight and jog-free segments of the transverse Poisson's response in the later stages of loading. Closer examination reveals a slight dip in the response of the cross-ply laminate with variable strength of the $90^{\circ}$ plies and resin-rich layer. Internal stress fields reveal no further vertical fracture accumulation along the final loading segment for this laminate, but the dip is directly linked to the initiation and stable growth of delamination at the $0^{\circ} / 90^{\circ}$ interface between some vertical cracks that had already formed, indicative of damage mode bifurcation hypothesized in the literature, and in fact observed by Paris et al. (2010b). Examination of internal stress fields in the remaining two cases reveals no evidence of delamination initiation. However, gradual vertical crack growth is observed in the laminate without resin-rich layer, resulting in additional vertical crack formation without sudden release of accumulated strain energy that would produce visible jogs in the homogenized response. In this case, vertical crack saturation is not attained in the considered loading range even though axial stress between two adjacent vertical cracks becomes negative. The delamination and gradual vertical crack growth phenomena are illustrated in greater detail later. 
The simulated through-thickness Poisson's response and crack density evolution with the applied laminate stress $\bar{\sigma}_{x x}$ for the three laminate types are shown in Figure 6.7b. The features of the homogenized through-thickness Poisson's response involving jogs that correspond to sudden vertical fracture events and dip that corresponds to the delamination initiation are similar to those observed in the transverse Poisson's response. The main difference is the smaller impact of the resin-rich layer and the $90^{\circ}$ ply strength variability on the homogenized response which manifests itself in smaller differences amongst the three simulated through-thickness Poisson's responses. The crack density evolution curves provide support for the foregoing discussion that relates vertical crack evolution to the features of the homogenized transverse Poisson's response. Specifically, continuous vertical crack accumulation is observed in the cross-ply laminate without resin-rich layer despite no apparent evidence of sudden fracture events seen in the homogenized Poisson's response in the later stage of loading characterized by a linear segment. In contrast, crack density saturation is observed in the remaining two cross-ply laminates. The simulations predict lower saturation crack density for the laminate with variable interface strength, 1.2 cracks $/ \mathrm{mm}$ versus $1.6 \mathrm{cracks} / \mathrm{mm}$ for the laminate with uniform interface strength, which is consistent with the literature. Because the experimental data for the considered crossply laminate does not include crack density evolution, the FVDAM theory's ability to capture this feature will be validated in the sequel using experimental data widely employed in the literature for this purpose. Nonetheless, comparison with the saturation crack density of 1.7 cracks/mm for a similar graphite/epoxy cross-ply laminate reported by Wang et al. (1984) provides confidence in the generated results.

Figure 6.8 illustrates the gradual formation of vertical cracks along the last segment of the transverse Poisson's response shown in Figure 6.7a characterized by straight line between steps 52 and 70 in the $\left[0 \frac{0}{2} / 90_{2}^{0}\right]_{s}$ laminate without resin-rich layer but with variable $90^{\circ}$ ply transverse strength. Axial through-thickness stress distributions are shown along those interfaces that have not failed up to step 52. The absence of resin-rich layer with lower elastic modulus produces high axial stress at the $0^{\circ} / 90^{\circ}$ interface where gradual crack growth initiates along a vertical interface with a lower strength relative to other intact interfaces. The crack progresses towards the laminate's midplane until complete fracture occurs. Partial vertical cracks along adjacent interfaces begin to form with increasing load until they too produce complete throughthickness fractures of the $90^{\circ}$ plies. 

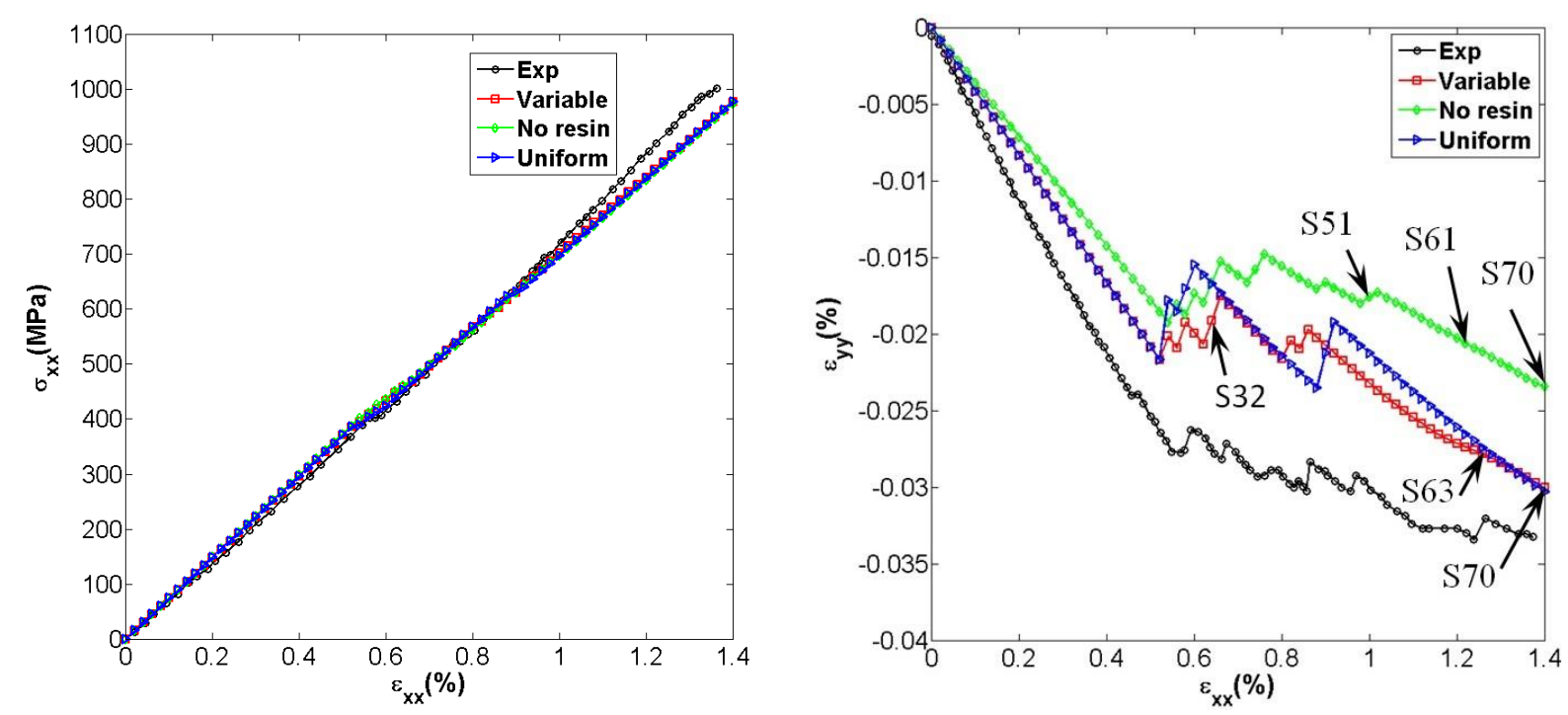

(a) Experimental vs simulated response
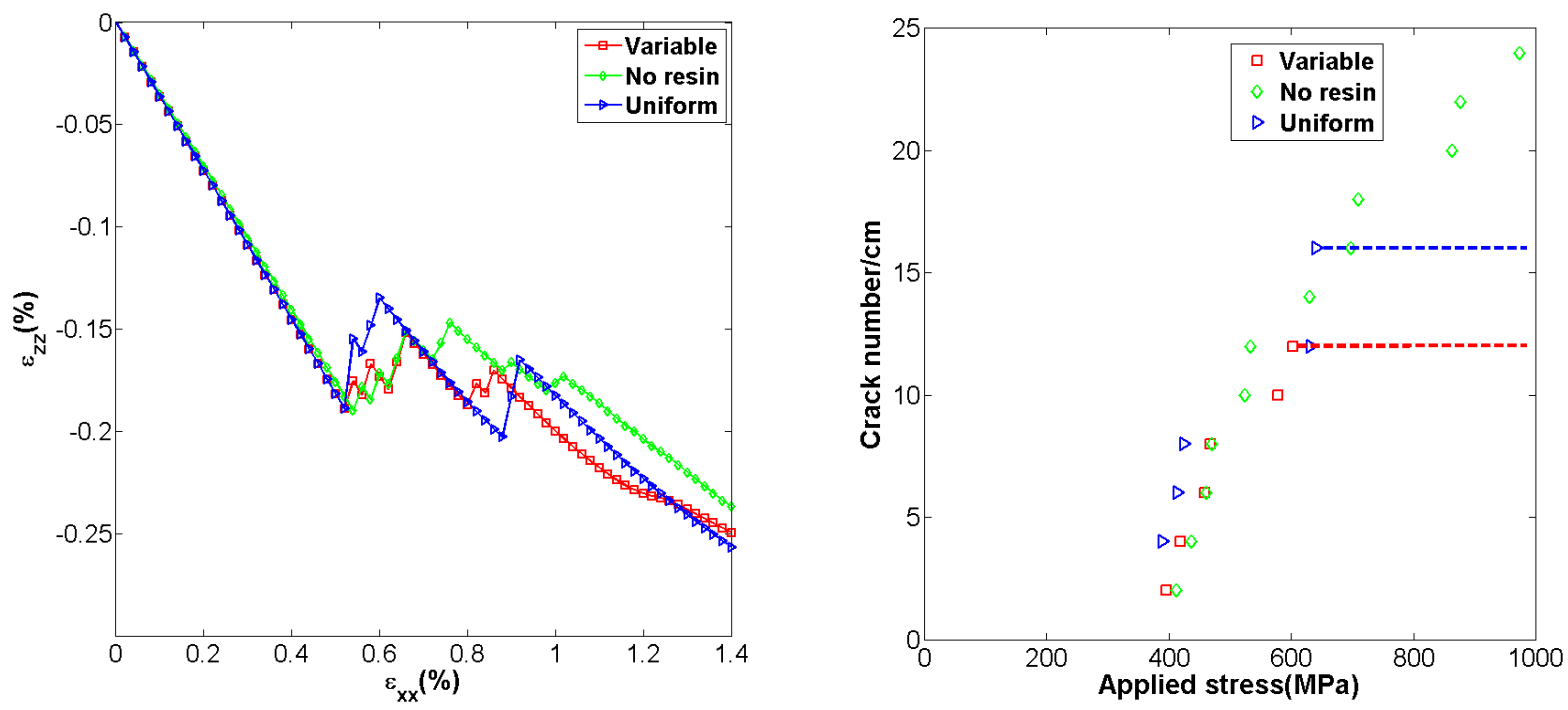

(b) Simulated response

Figure 6.7 Homogenized axial, transverse and through-thickness Poisson's responses of a $\left[0_{2}^{\circ} / 90_{2}^{\circ}\right]_{s}$ laminate under uniaxial loading, and crack evolution with applied stress, demonstrating the effects of resin-rich interfacial layer and transverse interface strength variability of the $90^{\circ}$ plies.

Table 6.4 Cohesive zone model parameters for the graphite-epoxy $\left[0_{2}^{0} / 90_{2}^{0}\right]_{s}$ laminate tested by (Pindera, 1986). Note: $k_{n}^{0}=\sigma_{\max } / \Delta_{n}^{0}$. The parameters for the $0^{0} / 90^{0}$ interface are based on the strain energy release rates of $G_{I C}=298 \mathrm{~J} / \mathrm{m}^{2}$ and $G_{I I C}=142 \mathrm{~J} / \mathrm{m}^{2}$, (Wilkins et al. 1982).

\begin{tabular}{|c|c|c|c|c|c|}
\hline Interface type & $\mathrm{k}_{\mathrm{n}}^{\mathrm{o}}=\mathrm{k}_{\mathrm{t}}^{\mathrm{o}}(\mathrm{GPa} / \mathrm{mm})$ & $\sigma_{\max }=\tau_{\max }(\mathrm{MPa})$ & $\Delta_{\mathrm{n}}^{\mathrm{o}}=\Delta_{\mathrm{t}}^{\mathrm{o}}(\mathrm{mm})$ & $\Delta_{\mathrm{n}}^{\mathrm{c}}(\mathrm{mm})$ & $\Delta_{\mathrm{t}}^{\mathrm{c}}(\mathrm{mm})$ \\
\hline $90^{\circ} / 90^{\circ}$ lower - upper & $9.65 \times 10^{2}$ & {$[7787]$} & {$[7.989 .02] \times 10^{-5}$} & {$[7.989 .02] \times 10^{-5}$} & {$[7.989 .02] \times 10^{-5}$} \\
\hline Epoxy resin & $4.20 \times 10^{2}$ & 69 & $1.64 \times 10^{-4}$ & $1.64 \times 10^{-4}$ & $1.64 \times 10^{-4}$ \\
\hline $0^{\circ} / 90^{\circ}$ & $4.20 \times 10^{2}$ & 50 & $1.19 \times 10^{-4}$ & $1.19 \times 10^{-2}$ & $5.68 \times 10^{-3}$ \\
\hline
\end{tabular}




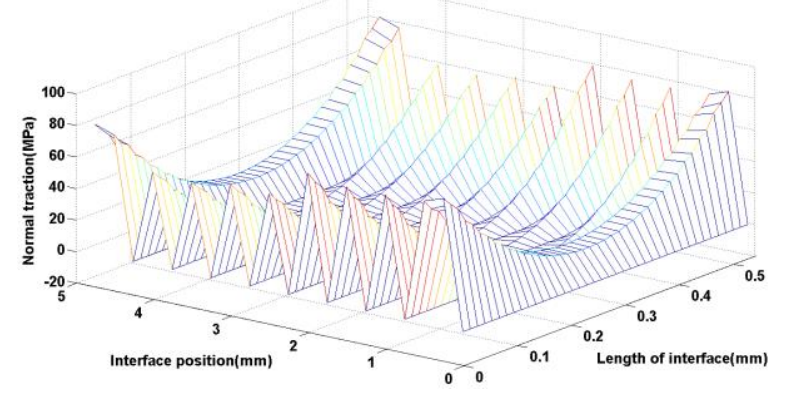

Step 51. Interfaces $1,5,7,9,11,13,15,17,19$ have failed catastrophically during loading to this step (see abrupt discontinuities in the tranverse Poisson's response in Figure 6.7.

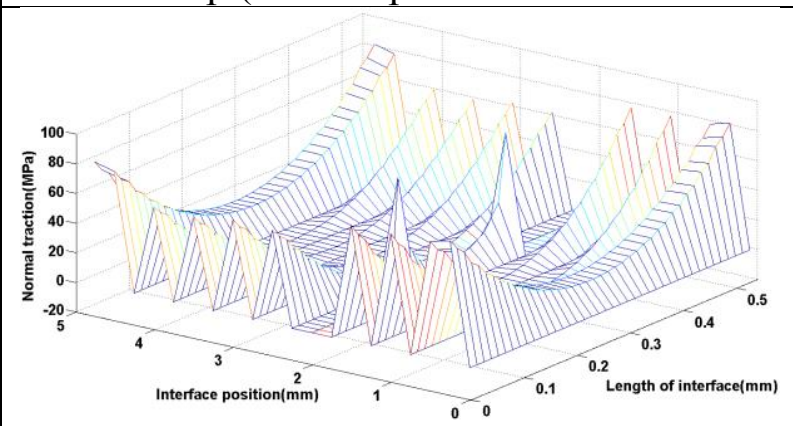

Step 53: Initiation of partial transverse crack

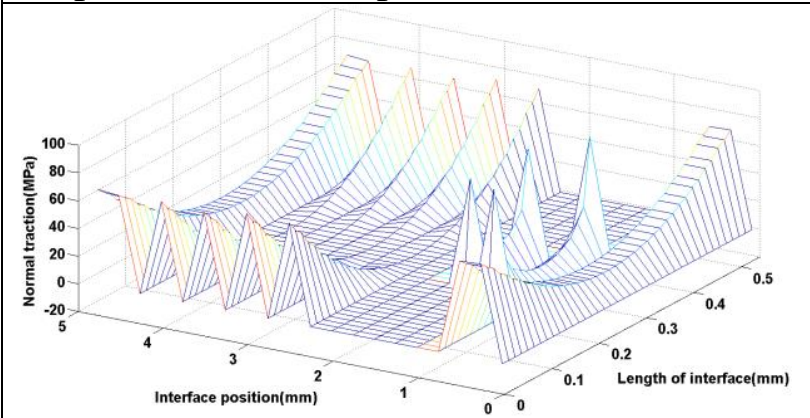

Step 62: Formation of complete transverse crack

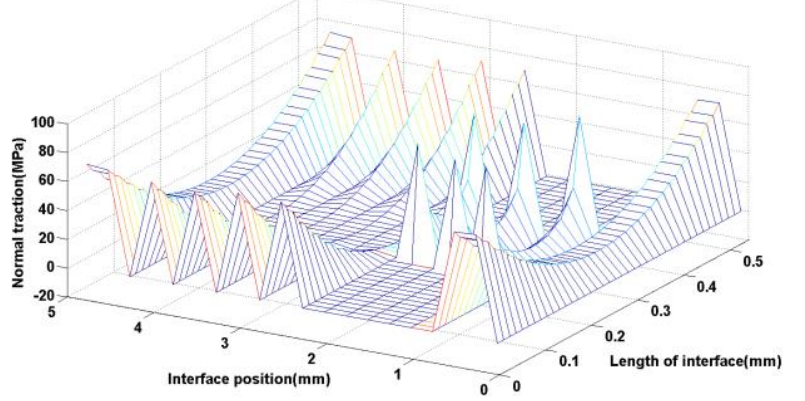

Step 61: Just before formation of vertical crack

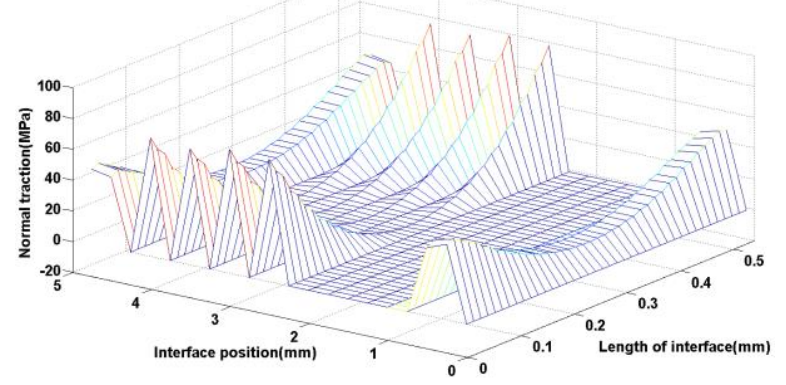

Step 70: Formation of additional complete transverse cracks

Figure 6.8 Through-thickness axial stress distributions along vertical interfaces that have not failed up to Step 51, illustrating gradual transverse crack progression with increasing load that does not produce a discontinuity in the transverse Poisson's response (see Figure 6.7a).

\subsubsection{Damage Mode Bifurcation}

Further to the foregoing discussion, Figure 6.9 illustrates the initiation and growth of delamination at the fourth vertical interface of the $\left[0 \frac{0}{2} / 90_{2}^{0}\right]_{s}$ laminate with variable transverse strength of the $90^{\circ}$ plies and resin-rich layer. The delamination initiation occurs at step 63 which corresponds to the start of gradual dip seen in the transverse Poisson's response in Figure 6.9a. Growth of the delamination is accompanied by increasing length of traction-free segment of the $0^{\circ} / 90^{\circ}$ interface, shifting of the interfacial tangential traction and the concomitant growth of the 
tangential displacement discontinuity. Included in the figure are the full-field stress distributions $\sigma_{x x}, \sigma_{x z}$ and $\sigma_{z z}$ in the region between two adjacent vertical cracks where delamination initiates and grows, beginning at step 32 at which vertical crack has formed in the considered location, i.e., at the fourth vertical interface. The delamination growth reduces axial stress transfer into the $90^{\circ}$ plies through interfacial shearing action as observed in the $\sigma_{x x}$ and $\sigma_{x z}$ distributions at steps 63 and 75.

The results in Figures 6.7-6.9 are based on the horizontal interface strength of $50 \mathrm{MPa}$ obtained from the strain energy release rate data reported by Wilkins et al. (1982), Table 6.4. Additional simulations were performed using horizontal interface strengths of 40 and $60 \mathrm{MPa}$ to determine the effect of this parameter on the damage mode transition from vertical cracking of the inner $90^{\circ}$ plies to horizontal delamination of the $0^{\circ} / 90^{\circ}$ interface. As the horizontal interface strength was decreased and increased from the reference strength of $50 \mathrm{MPa}$, the remaining cohesive zone model parameters were adjusted accordingly in order to keep the critical energy release rate $G_{I I C}$ unchanged. The ensuing homogenized transverse Poisson's responses are compared in Figure 6.10 together with the vertical crack evolution. The simulation based on the lowest horizontal interface strength indicates that substantial interface degradation occurs at a low applied stress, as suggested by the gradual change in the transverse Poisson's response, leading to subsequent delamination initiation and stable growth at lower applied stress relative to the response based on the interface strength of $50 \mathrm{MPa}$. Hence vertical crack saturation also occurs at a lower applied stress. Increasing the horizontal interface strength to $60 \mathrm{MPa}$ suppresses horizontal interface degradation and promotes unstable delamination growth when the interfacial strength is achieved. This is observed in the homogenized transverse Poisson's response which remains piece-wise linear until delamination instability occurs at approximately the same applied stress that initiates stable delamination growth for the $50 \mathrm{MPa}$ horizontal interface. The simulation was terminated at this point.

Finally, the results in Figures 6.7-6.9 were generated using a particular random strength distribution for the vertical interfaces in the [77 87] MPa interval. Additional simulations with different vertical strength distributions and horizontal interface strength of $50 \mathrm{MPa}$ indicate that crack density saturation occurs at the same level around 1.2 cracks/mm and roughly the same applied axial stress, and delamination initiation and stable growth occur in the same loading history interval, despite differences in fracture locations in the early stages of loading. 

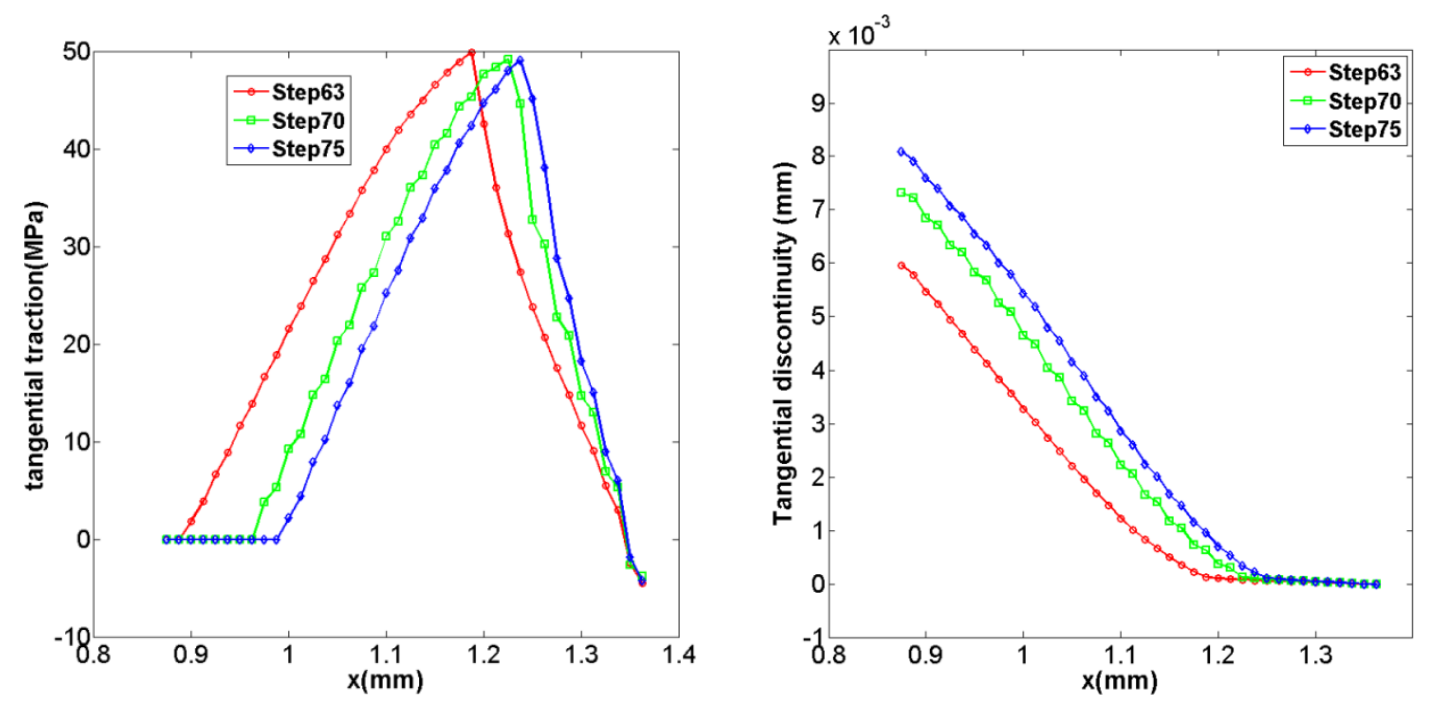

(a)

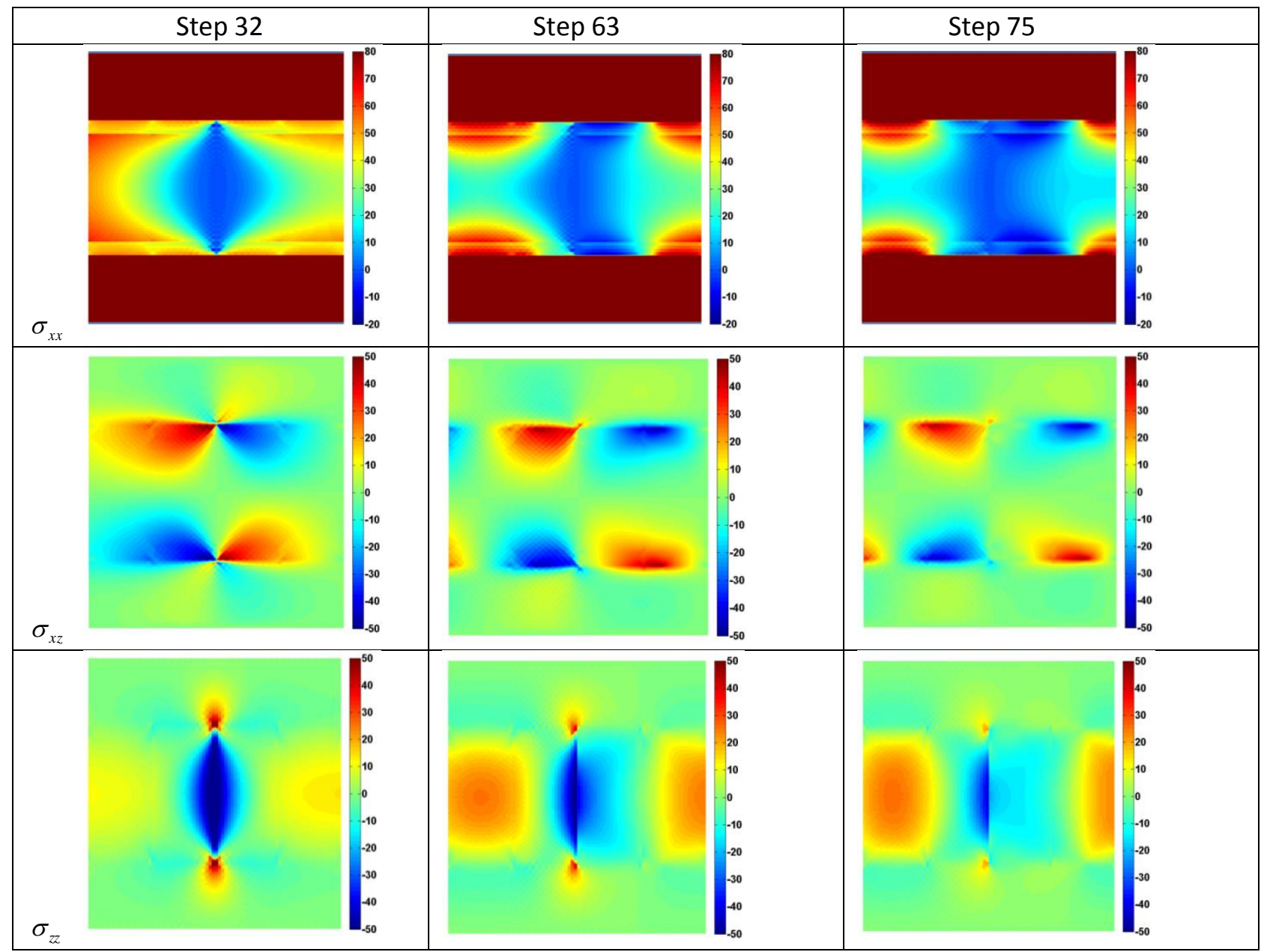

(b)

Figure 6.9 (a) Initiation and progression of delamination along the $0^{\circ} / 90^{\circ}$ interface of a $\left[0_{2}^{\circ} / 90_{2}^{\circ}\right]_{s}$ laminate in the vicinity of a vertical crack in the $90^{\circ}$ plies; (b) corresponding stress fields. 

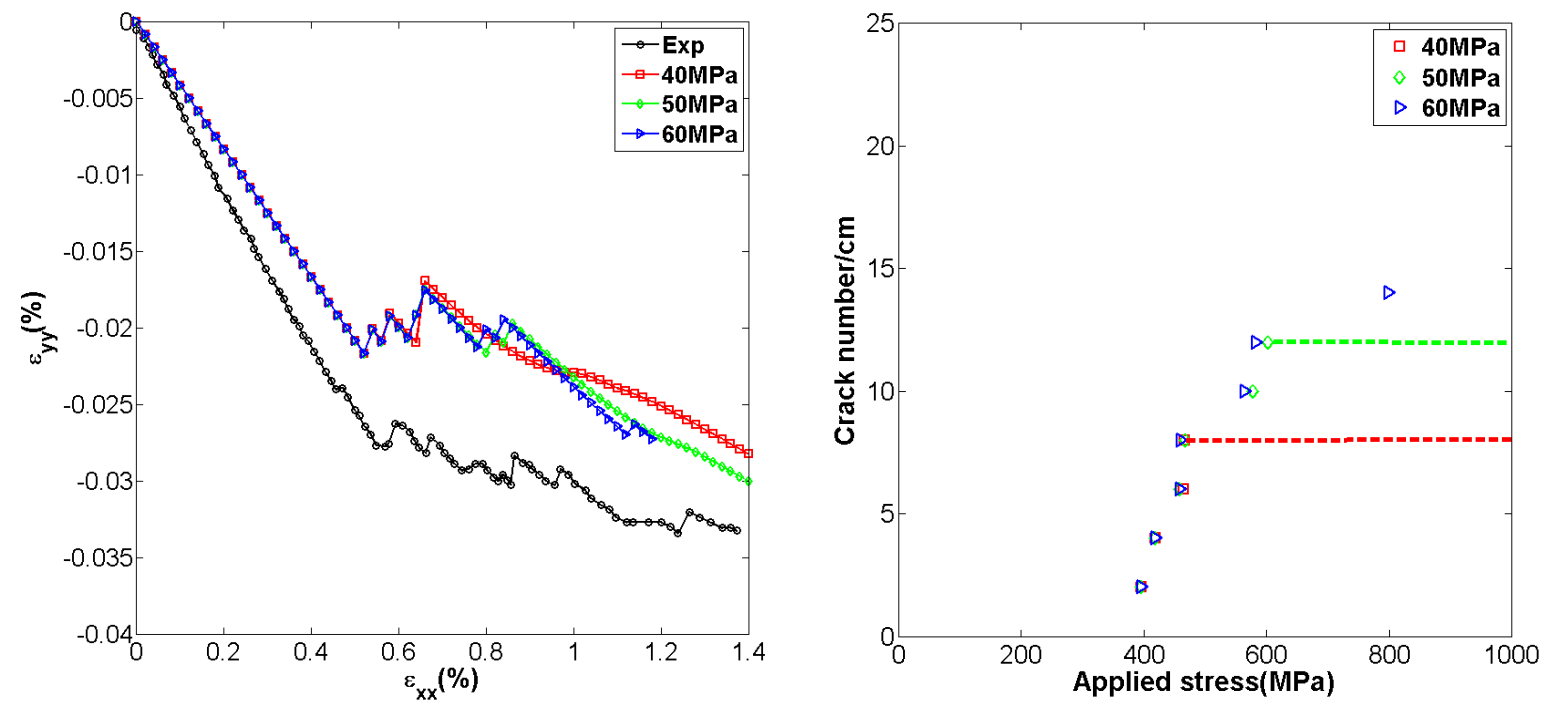

Figure 6.10 Homogenized transverse Poisson's response of a $\left[0_{2}^{\circ} / 90_{2}^{\circ}\right]_{s}$ laminate under uniaxial loading, and crack evolution with applied stress, demonstrating the effects of horizontal interface strength variability of the $90^{\circ}$ plies.

\subsection{Crack Density Evolution - Theory vs Experiment}

The simulated crack density evolution for the cross-ply laminate of the preceding section cannot be verified directly due to the lack of experimental data. The best that can be concluded is that the events observed in the simulated homogenized transverse Poisson's response are directly related to fracture events in the $90^{\circ}$ plies which correlate well with the recorded experimental data. Hence in this section we directly compare the crack density evolution experimentally recorded by Wang et al. (1985) with FVDAM simulations for two laminates, namely $\left[0_{2}^{0} / 90^{0}\right]_{S}$ and $\left[0_{2}^{0} / 90_{2}^{0}\right]_{S}$ with the latter the same as the lay-up of the preceding section. Comparison of the ply-level thermo-elastic moduli of the two material systems given in Tables 6.2 and 6.3 reveals their similarity. To simulate the fabrication-induced residual stresses, temperature cooldown of $125^{\circ} \mathrm{C}$ was first applied, following Wang et al. (1985), subsequent to mechanical loading. The cohesive zone model parameters for Wang's data are given in Table 6.5. The lower bounds on the in-situ $90^{\circ}$ ply strengths were calculated using the experimentally observed occurrence of firstply failure for both laminates, and subsequent FVDAM simulations were performed using random vertical interface strength distributions with the range of $10 \mathrm{MPa}$ following the range used in the preceding section. 
Figure 6.11 presents comparison of the simulated crack density evolution with experimental data for the two cross-ply laminates. For each laminate type, the simulations were performed using two strengths for the $0^{\circ} / 90^{\circ}$ horizontal interface of 65 and $200 \mathrm{MPa}$, and the same random strength distributions for the vertical interfaces. Hence the differences in crack density evolution for each laminate type are due to the differences in horizontal interfacial degradation, with the highest strength of $200 \mathrm{MPa}$ producing interfacial separations within the elastic range. In the case of the $\left[02 / 90^{\circ}\right]_{S}$ laminate, little difference is observed between simulations based on the two horizontal interfacial strengths throughout almost the entire loading range, suggesting that no damage mode bifurcation occurs in the considered loading range. Examination of the interfacial tractions indicates some degradation of the weaker horizontal interface, albeit without complete separation or delamination, while the stronger interface continues to load in the elastic range. For the weaker interface, the rate of crack density evolution decreases dramatically beyond the applied stress of around $800 \mathrm{MPa}$ which corresponds to 1.6 cracks $/ \mathrm{mm}$, increasing slowly to $1.8 \mathrm{cracks} / \mathrm{mm}$ just before laminate failure at the axial strain of $1.23 \%$. The laminate with the stronger interface experiences similar crack evolution until the last stage of loading that initiates at $1.8 \mathrm{cracks} / \mathrm{mm}$. Beyond this point, a rapid and continuous vertical crack increase is observed until failure. Simulations based on both horizontal interface strengths produce crack density evolution which closely follows the experimental results of Wang et al. (1985).

Greater differences in the crack density evolutions due to the different horizontal interface strengths are observed in the simulations of the $\left[02_{2}^{0} / 90_{2}^{0}\right]_{s}$ laminate response. While crack density saturation at around $1.8 \mathrm{cracks} / \mathrm{mm}$ is observed for the lower interfacial strength at the stress level substantially below the laminate failure stress, continued vertical crack growth with applied stress occurs for the larger strength which does not saturate up to the maximum applied stress. As in the preceding case, degradation of the horizontal interface without delamination does occur for the weaker interface while the stronger interface loads elastically. The simulated crack density evolutions follow the experimental data quite well.

To gain additional insight into the vertical crack evolution in the above two configurations following the preceding section's results, the corresponding transverse Poisson's responses were also simulated, Figure 6.12. The simulations show that increasing the content of $90^{\circ}$ plies substantially decreases the transverse Poisson's response while increasing the severity 
of vertical fracture events due to the greater constraining effect of the transverse plies. The simulated transverse Poisson's response of Wang's $\left[0_{2}^{0} / 90_{2}^{0}\right]_{s}$ laminate is very similar to the corresponding laminate investigated in the preceding section, but does not exhibit gradual dips associated with interfacial delaminations.

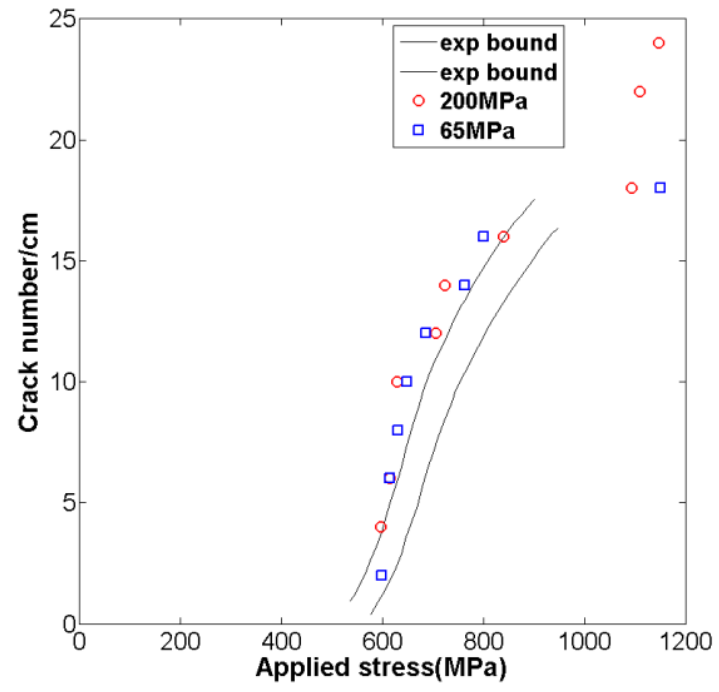

$\left[0_{2}^{\circ} / 90^{\circ}\right]_{s}$

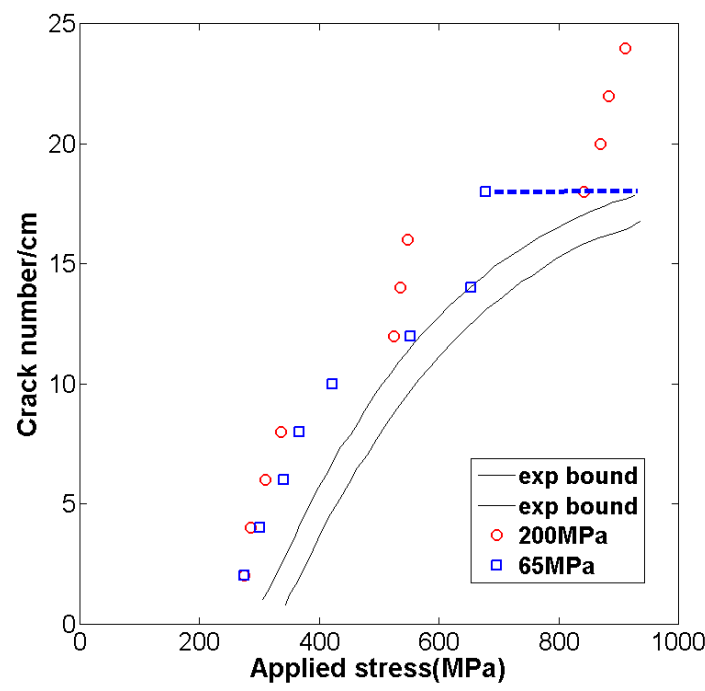

$\left[0_{2}^{\circ} / 90_{2}^{\circ}\right]_{s}$

Figure 6.11 Crack density evolution as a function of applied axial stress in cross-ply laninates with different thickness of the inner $90^{\circ}$ plies tested by Wang et al. (1985) under uniaxial loading.

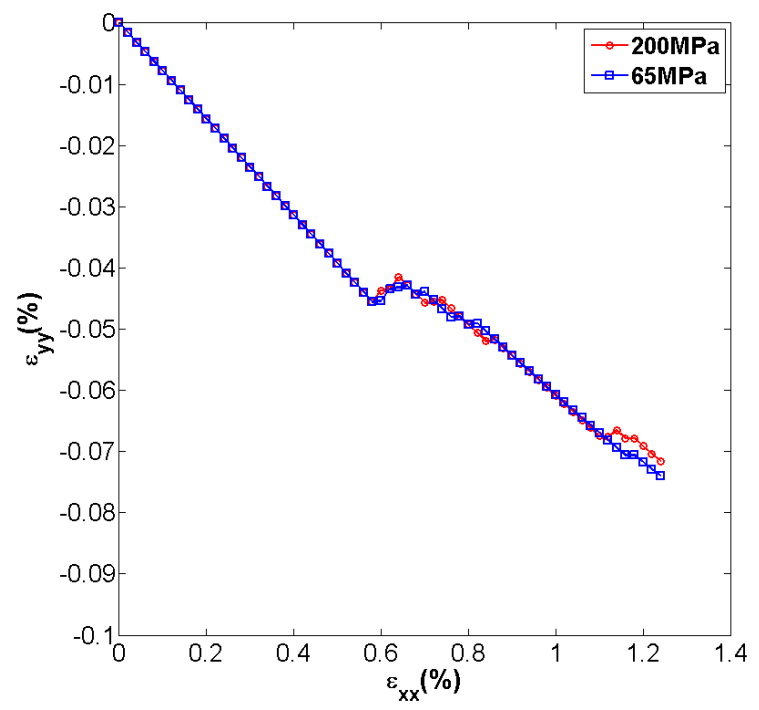

$\left[0_{2}^{\circ} / 90^{\circ}\right]_{s}$

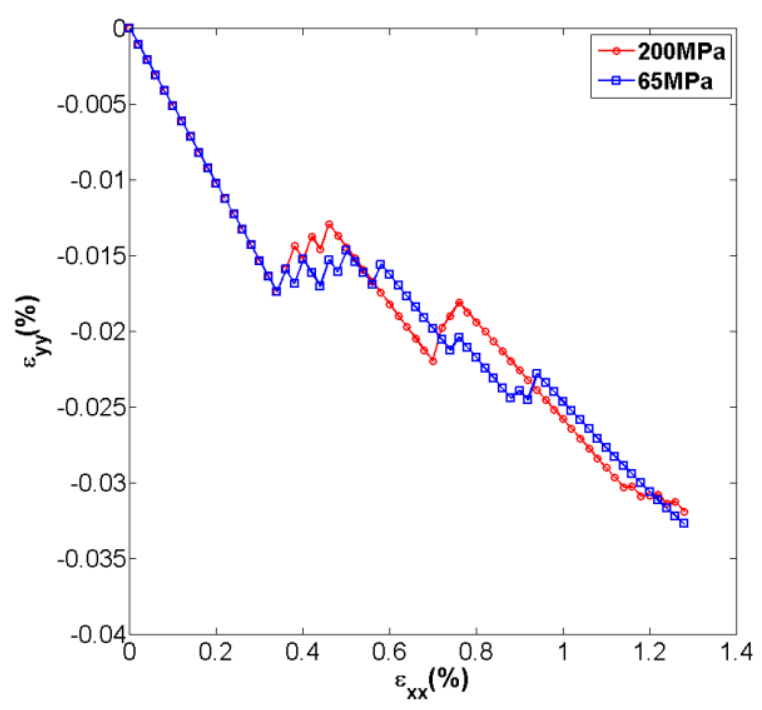

$\left[0_{2}^{\circ} / 90_{2}^{\circ}\right]_{s}$

Figure 6.12 Predicted homogenized transverse Poisson's responses of cross-ply laminates with different thickness of the inner $90^{\circ}$ plies tested by Wang et al. (1985) uniaxial loading. 


\subsection{Discussion}

The simulation of damage evolution on the fly in cross-ply laminates is a relatively recent endeavor enabled by the cohesive zone model. While transverse cracking in this class of laminates has been investigated recently by Shi et al. (2014) using the cohesive zone model approach within the finite-element framework, the authors are not aware of an investigation addressing dynamic transition from vertical crack evolution in $90^{\circ}$ plies to interfacial $0^{\circ} / 90^{\circ}$ delamination. This is in contrast with the still-life analyses of damage mode evolution tendency that involve calculation of energy release rates associated with vertical crack growth and subsequent delamination emanating from the vicinity of the vertical crack tip, (Wang et al. 1985, Dharani et al. 2003). The study of Paris et al. (2010a,b) on the different scenarios associated with the mechanics of vertical crack growth and subsequent delamination at, or parallel to, the $0^{\circ} / 90^{\circ}$ interface is the most recent example of this approach which provided insight into the importance of microstructural details based on experimental evidence. Nonetheless, the question of damage mode bifurcation in cross-ply laminates remains open in the absence of an accepted criterion for crack deflection along a bi-material interface. While the calculation of strain energy release rates can be carried out for a deflected crack along the bi-material interface, an initial crack size is required for the calculation to proceed. Because the singular stress field at the bi-material interface changes upon crack deflection, it is not easy to calculate strain energy release rate associated with damage mode bifurcation which would provide a basis for formulating an energy-based criterion. The alternative is to use the cohesive zone model as a vehicle to determine under what conditions such bifurcation is possible in the absence of an assumed flaw/crack size. This model's parameters are also based on critical energy release rates but these rates are associated with the different crack propagation modes. Hence it may be argued that the preference of one over the other is based on the overall equilibrium considerations satisfied in the course of the boundary-value problem solution.

The dynamic damage evolution simulations proved useful in revealing the dramatic effect of vertical cracking on the laminate transverse Poisson's response that depends on the $90^{\circ}$ ply content. While the effect of crack density on the transverse Poisson's ratio is known to be

substantially more dramatic than on the axial Young's modulus, Smith and Wood (1990), there is 
virtually no experimental data on the transverse strain vs axial strain response of cross-ply laminates which is an excellent indicator of evolving damage.

\subsection{Conclusions}

In this contribution we have examined the evolution of two types of damage modes in polymeric matrix cross-ply laminates and their effect on the homogenized response using a recently developed cohesive zone model-based homogenization theory for periodic materials. The theory naturally enables simulation of damage mode evolution in cross-ply laminates on the fly without the use of a damage bifurcation criterion typically employed in the literature. The quantity that controls damage evolution is the critical strain energy release rate associated with the different damage modes which, in turn, is characterized by three cohesive zone model parameters. These parameters naturally control damage mode transition from transverse cracking of the $90^{\circ}$ plies to delamination of the $0^{\circ} / 90^{\circ}$ horizontal interface. The outcomes of finite-volume simulations are homogenized responses in the presence of evolving damage that are generated on the fly as the load increases, internal stress fields and details of damage evolution. Comparison of the simulations with experimental response of graphite/epoxy cross-ply laminates leads to the following conclusions:

- Damage evolution events are much more pronounced (and observable) in the homogenized transverse Poisson's response than the axial response throughout the entire loading range: large jogs in the transverse Poisson's response are directly related to the occurrence of abrupt vertical cracks in the $90^{\circ}$ plies, which are readily observed in the axial response at first-ply failure but not subsequent failure events

- Damage events may be related to the type of damage: while vertical cracks in the $90^{\circ}$ plies are characterized by abrupt jogs in the transverse Poisson's response during initial loading stages, progressive or stable delamination of the $0^{\circ} / 90^{\circ}$ horizontal interface is characterized by a gradual slope change

- Gradual vertical crack growth has been observed in later stages of loading characterized by linear transverse Poisson's response in the absence of resin-rich region at the $0^{\circ} / 90^{\circ}$ horizontal interface and horizontal interface delamination 
- Vertical crack density saturation is influenced by the $90^{\circ}$ ply strength variability and the presence of resin-rich layer at the $0^{\circ} / 90^{\circ}$ horizontal interface

- Damage mode bifurcation has been observed to occur from vertical crack evolution in the $90^{\circ}$ plies to progressive delamination of the $0^{\circ} / 90^{\circ}$ horizontal interface in the case of the $\left[0_{2}^{0} / 90_{2}^{0}\right]_{s}$ laminate with variable vertical interface strength, proper choice of horizontal interface strength and presence of resin-rich layer

Comparison of the finite-volume simulations of crack density evolution with experimental data on similar graphite/epoxy cross-ply laminates with different $90^{\circ}$ ply proportions supports the above observations and provides additional insight into the effect of $90^{\circ}$ ply thickness, which substantially enhances the effect of vertical cracks on the transverse Poisson's response. 


\section{Chapter 7}

\section{Off-Axis Loading}

\subsection{Introduction}

Polymeric matrix composites are known to exhibit substantial inelastic behavior under off-axis loading which is often employed to characterize the response of unidirectional laminae. Four of the five elastic moduli that define the elastic response of transversely isotropic materials are obtained from the so-called off-axis test of unidirectional plies, including the axial shear modulus $G_{12}$ obtained from the $10^{0}$ off-axis specimen. The inelastic behavior is typically more pronounced in the presence of shear stress in the principal material coordinate system, which is the dominant stress component that governs matrix-dominated inelastic response in low off-axis specimen configurations. The nature of the inelastic response at the homogenized level in polymeric matrix composites is not always clear, unlike metal matrix composites, and accordingly has been simulated by viscoplastic, elastic-plastic and viscoelastic constitutive models. Approaches based on the assumption that inelastic response is due to damage evolution are rare.

In this chapter we consider a polymeric matrix unidirectional composite, graphite/polyimide, that was developed by NASA in the 1980's for the now-decommissioned space shuttle cargo bay door. The polyimide resin was developed for elevated temperature exposures up to $600^{\circ} \mathrm{F}$, and exhibits an essentially linearly elastic response at room temperature in tension, compression and shear, characterized by brittle fracture. When tested along different directions relative to the fiber orientation, the unidirectional composite containing approximately 60\% Celion 6000 graphite fibers stiffens under tensile loading along the fiber direction and remains nearly elastic under transverse tension perpendicular to the fiber direction, failing catastrophically in a brittle fashion. In contrast, under off-axis loading for low off-axis configurations, substantial nonlinearity and dissipation are observed that are traced to the inelastic response in shear in the principal material coordinate system. Figure 7.1 summarizes these different response characteristics. 
In this chapter, the off-axis inelastic response of the graphite/polyimide specimens is simulated based on the assumption that the observed nonlinearity is largely due to the fiber/matrix interfacial sliding arising from the significant inplane shear stress component in the principal material coordinate system. The interfacial sliding is simulated by the cohesive zone model implemented for off-axis loading. The advantage of the developed CZM-based FVDAM theory over a comparable finite-element simulation using a commercial code such as Abaqus is the FVDAM's ability to model the combined out-of-plane response of unidirectional composites without resorting to a full three-dimensional model. In this chapter, the full capability of the developed FVDAM theory is displayed as the simulation of the inelastic response caused by evolving fiberlmatrix interfacial damage requires knowledge of the unknown elastic moduli of the fiber and matrix phases, CZM parameters, and the effect of residual stresses. Towards this end, the FVDAM-based PSO algorithm is employed as the first step in simulating the interfacial damage-induced inelastic response.

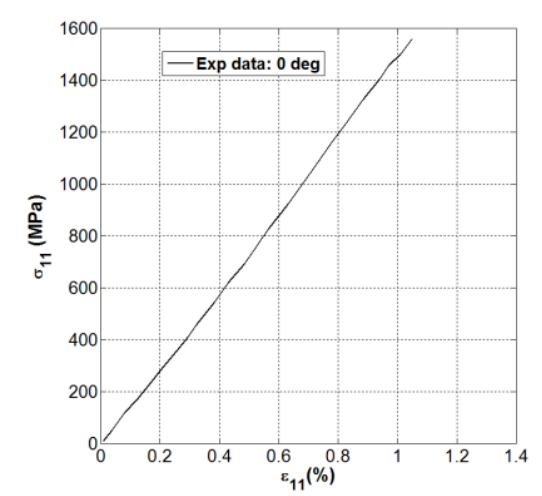

(a)

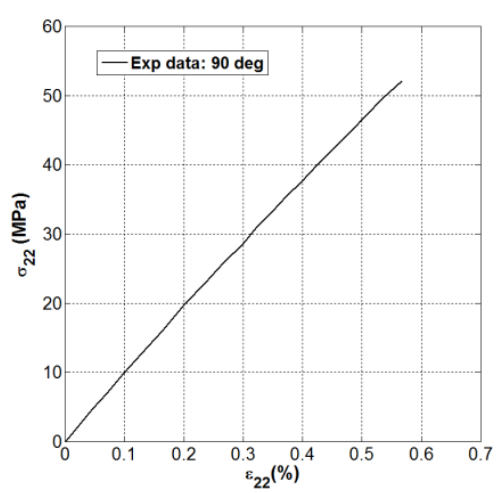

(b)

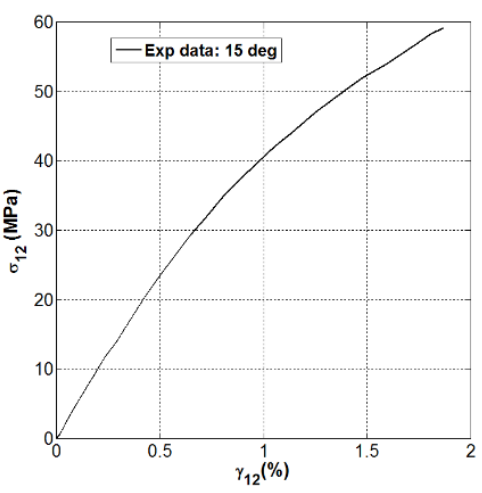

(c)

Figure 7.1 Pure tensile response along the fiber direction (a) and perpendicular to the fiber direction (b), and shear response in the principal material coordinate system of a $15^{0}$ off-axis specimen subjected to uniaxial loading in the laminate coordinate system (c).

\subsection{Implementation of Off-Axis Loading}

Figure 7.2 illustrates a specimen with the fiber direction rotated by an angle $\theta$ about the $x_{3}$ axis, uniaxially loaded by the normal inplane stress $\sigma_{x x}$, or $\boldsymbol{\sigma}_{x}=\left[\begin{array}{lll}\sigma_{x x} & 0 & 0\end{array}\right]$ with reference to the global coordinate system $(\mathrm{x}, \mathrm{y}, \mathrm{z})$. In the principle material coordinate $\operatorname{system}\left(x_{1}, x_{2}, x_{3}\right)$, the material experiences a combined state of plane stress characterized by $\sigma_{11}, \sigma_{12}$ and $\sigma_{22}$ or $\boldsymbol{\sigma}=$ [ $\left.\sigma_{11} \sigma_{12} \sigma_{22}\right]$. The $x_{1}$ axis is along the fiber direction, $x_{2}$ is perpendicular to the fiber direction in the specimen's plane, and $x_{3}$ is the out-of-plane axis. The in-plane stress and engineering strain 
in two coordinates systems are related by the familiar transformation relations $\sigma=\mathbf{T}_{1} \boldsymbol{\sigma}_{x}$ and $\boldsymbol{\varepsilon}=\mathrm{T}_{2} \boldsymbol{\varepsilon}_{x}$, where $\mathbf{T}_{1}$ and $\mathbf{T}_{2}$ are the transformation matrices for coordinate rotation by $\theta$ about the $x_{3}$ axis (Herakovich, 1998).

The ability to simulate crack initiation and propagation in a unidirectional composite subjected to off-axis loading sets FVDAM apart from other micromechanics models, and particularly from the readily available commercial finite-element codes that require a threedimensional unit cell analysis to accommodate the out-of-plane shear stress that arises under such loading. In contrast, the two-dimensional micromechanics framework described in Chapter 2 is valid for any combination of macroscopic stress and strain components, including the out-ofplane contributions. Eq. (2.33) defines the macroscopic strain path for the given macroscopic stresses in principal material coordinates. The adaptation of Eq. (2.33) to off-axis loading is done in the following manner.

First, the effective stiffness matrix $\mathbf{C}^{*}$ is calculated in the principal material coordinate system for a given unit cell irrespective of the applied loading. Then the effective stiffness matrix $\overline{\mathbf{C}}^{*}$ in the global coordinate system, in which loading is applied, is calculated via the transformation law $\overline{\mathbf{C}}^{*}=\mathbf{T}_{1} \mathbf{C}^{*} \mathbf{T}_{2}^{-1}$. With the aid of the effective stiffness matrix $\overline{\mathrm{C}}^{*}$ in the global coordinate system, the total macroscopic $\overline{\boldsymbol{\varepsilon}}_{x}$ strain components can be determined via the transformed macroscopic constitutive equation

$$
\overline{\boldsymbol{\sigma}}_{x}=\overline{\mathbf{C}}^{*} \overline{\boldsymbol{\varepsilon}}_{x}-\left(\overline{\boldsymbol{\sigma}}^{T}+\overline{\boldsymbol{\sigma}}^{d}\right)_{x}
$$

in which a combination of any six components of the vector $\left\{\overline{\boldsymbol{\sigma}}^{T}+\overline{\boldsymbol{\sigma}}^{d}\right\}_{x}$ is obtained from $\left(\overline{\boldsymbol{\sigma}}^{T}+\overline{\boldsymbol{\sigma}}^{d}\right)_{x}=T_{1}^{-1}\left(\overline{\boldsymbol{\sigma}}^{T}+\overline{\boldsymbol{\sigma}}^{d}\right)$, where $\overline{\boldsymbol{\sigma}}^{T}+\overline{\boldsymbol{\sigma}}^{d}$ are the macroscopic thermo-inelastic contributions in the principal material coordinate system. Once all six components of the total macroscopic strain in the global coordinate system are obtained, the components of the total macroscopic strain in the principal material coordinate system are calculated using the transformation equations $\overline{\boldsymbol{\varepsilon}}=\mathrm{T}_{2} \overline{\boldsymbol{\varepsilon}}_{x}$, which are used directly in the global primary and auxiliary systems of equations. 


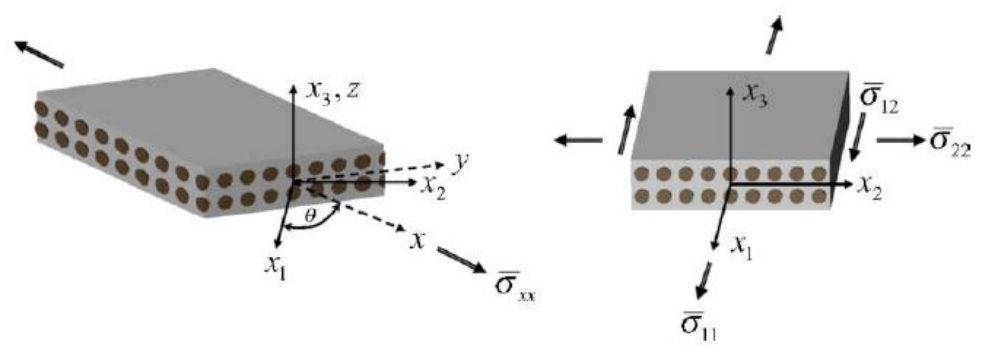

Figure 7.2 An off-axis specimen uniaxially loaded in the global coordinate and the corresponding transformed combined in-plane stresses in the principal material coordinate system.

\subsection{Determination of Fiber and Matrix Properties}

The material system mentioned in the Introduction under investigation in this chapter is composed of Celion 6000 graphite fibers developed by Celanese Corporation and a hightemperature polyimide system PMR-15 developed at NASA-Lewis. The nominal fiber volume fraction is $60 \%$ and the recorded volume fractions range from $59.77 \%$ to $62.5 \%$ (Pindera 1981). The averaged value of the recorded volume fraction is $61 \%$, which is used in the present analysis. The glass transition temperature for the material system is $327^{\circ} \mathrm{C}$ (Pindera 1981). Figure 7.3 illustrates the geometry and discretization of the hexagonal unit cell employed in the simulations which produces transversely isotropic behavior in the elastic range.

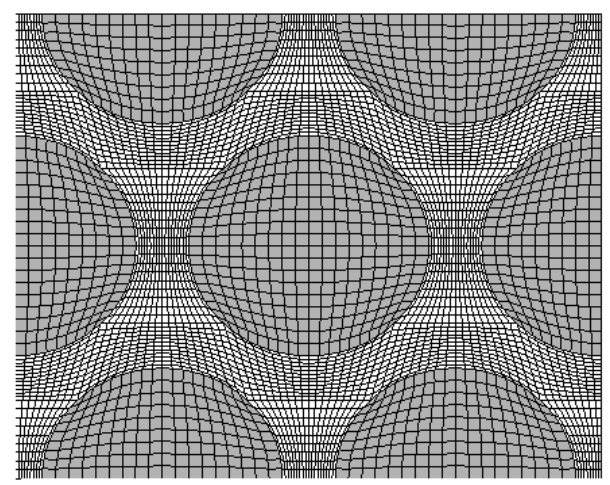

Figure 7.3 Hexagonal unit cell with volume fraction of $61 \%$, which is the average value of the recorded volume fraction.

The ply properties are transversely isotropic, which had been characterized by Pindera (1981) except for the transverse shear modulus $G_{23}$ and the Poisson's ratio $v_{23}$, Table 7.1. The Celion 6000 fiber properties are assumed transversely isotropic, but had not been measured. PMR-15 resin is considered to be isotropic and different matrix properties of PMR-15 have been reported. The material properties in Table 7.2 have been reported by Pindera (1981), while Jones 
reports a different Young's modulus, 4.26GPa. From the above discussion, it is clear that there are a lot of uncertainties about the fiber and matrix properties. Hence the purpose of this section is to determine the fiber properties of $E_{22}, v_{23}, G_{12}, v_{12}$ and matrix Young's modulus, $E_{m}$ by minimizing the difference between the homogenized properties generated from FVDAM simulations and the tested ply properties, Eq. (7.2). After determining the unknown fiber and matrix properties, the missing ply property $G_{23}$ or $v_{23}$ then can be calculated using FVDAM.

Table 7.1 Ply properties of Celion 6000/PMR-15 (Pindera, 1981).

\begin{tabular}{|c|c|c|c|c|}
\hline$E_{11}(\mathrm{GPa})$ & $E_{22}(\mathrm{GPa})$ & $G_{12}(\mathrm{GPa})$ & $G_{23}(\mathrm{GPa})$ & $v_{12}$ \\
\hline 136.6 & 9.8 & 5.0 & ---- & 0.35 \\
\hline
\end{tabular}

Table 7.2 PMR-15 matrix properties (Pindera, 1981) and the thermal expansion coefficients (Papadopoulos and Bowles, 1990).

$$
\begin{aligned}
& \begin{array}{|c|c|c|c|}
\hline \mathrm{E}(\mathrm{GPa}) & v & \mathrm{G}(\mathrm{GPa}) & \alpha\left(\mu /{ }^{\circ} \mathrm{C}\right) \\
\hline 3.13 & 0.391 & 1.13 & 36 \\
\hline
\end{array} \\
& E R R=\frac{1}{4}\left(\frac{\left|E_{11}^{*}-E_{11}^{\text {exp }}\right|}{E_{11}^{\text {exp }}}+\frac{\left|E_{22}^{*}-E_{22}^{\text {exp }}\right|}{E_{22}^{\text {exp }}}+\frac{\left|G_{12}^{*}-G_{12}^{\text {exp }}\right|}{G_{12}^{\text {exp }}}+\frac{\left|v_{12}^{*}-v_{12}^{\text {exp }}\right|}{v_{12}^{\text {exp }}}\right)
\end{aligned}
$$

Before characterizing the fiber and matrix properties based on the implemented PSO algorithm, reasonable ranges must first be assigned to the design variables. Because there is little data on the elastic moduli of Celion 6000 fibers, the elastic properties of the much better characterized T300 graphite fibers are often employed instead, (Papadopoulos and Bowles's 1990, Ran et al. 2014, Lu and Hutchinson 1995). These properties are listed in Table 7.3. In the current work, the fiber properties of T300 are treated as the reference points for the design variables. The lower bounds on the design variables are $20 \%$ lower that the reference points and the upper bounds on the design variables are $20 \%$ higher than the reference points, Table 7.4, but the Poisson's ratio cannot exceed 0.5. If it is exceeded during the optimization process, then the upper limit is reset to 0.49 .

Table 7.3 T300 fiber properties (Pindera 1981) and thermal expansion coefficients (Papadopoulos and Bowles, 1990).

\begin{tabular}{|l|c|c|l|c|l|l|}
\hline$E_{11}(\mathrm{GPa})$ & $E_{22}(\mathrm{GPa})$ & $v_{23}$ & $G_{12}(\mathrm{GPa})$ & $v_{12}$ & $\alpha_{A}\left(\mu /{ }^{\circ} \mathrm{C}\right)$ & $\alpha_{T}\left(\mu /{ }^{\circ} \mathrm{C}\right)$ \\
\hline 227.5 & 22.4 & 0.45 & 22.1 & 0.41 & -0.54 & 10.08 \\
\hline
\end{tabular}


Table 7.4 Design variables range for Celion 6000 fiber properties (20\%).

\begin{tabular}{|c|c|c|c|c|c|c|}
\hline & $E_{11}(\mathrm{GPa})$ & $E_{22}(\mathrm{GPa})$ & $v_{23}$ & $G_{12}(\mathrm{GPa})$ & $v_{12}$ & $E_{m}$ \\
\hline Lower bound & 182 & 17.9 & 0.36 & 17.7 & 0.33 & 3.13 \\
\hline Upper bound & 273 & 26.9 & 0.49 & 26.5 & 0.49 & 4.26 \\
\hline
\end{tabular}

During the optimization procedure, 20 particles are used and the maximum iteration number is 15. As shown in Figure 7.4, the optimization process converges at step 12 and the difference between homogenized properties and test data is only $0.2 \%$ according to the definition of Eq. (7.2). Table 7.6 presents comparison between test data and homogenized properties based on the optimal design variables in Table 7.5, showing that the homogenized properties are identical with the available test data. These fiber and matrix properties backed-out via optimization are used in all the following simulations.

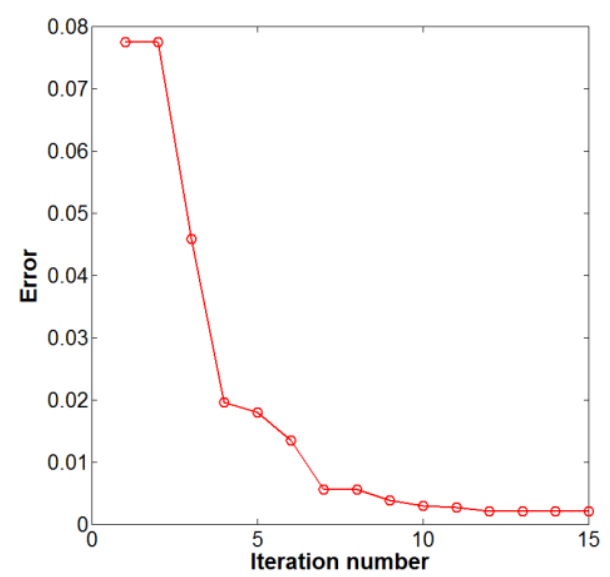

Figure 7.4 Convergence curve.

Table 7.5 Optimized design variables.

\begin{tabular}{|c|c|c|c|c|c|}
\hline$E_{11}(\mathrm{GPa})$ & $E_{22}(\mathrm{GPa})$ & $v_{23}$ & $G_{12}(\mathrm{GPa})$ & $v_{12}$ & $E_{m}(\mathrm{GPa})$ \\
\hline 221.6 & 17.9 & 0.49 & 26.1 & 0.33 & 4.1 \\
\hline
\end{tabular}

Table 7.6 Test data and homogenized properties based on optimized material properties.

\begin{tabular}{|c|c|c|c|c|c|}
\hline & $E_{11}(\mathrm{GPa})$ & $E_{22}(\mathrm{GPa})$ & $G_{12}(\mathrm{GPa})$ & $G_{23}(\mathrm{GPa})$ & $v_{12}$ \\
\hline Testing & 136.6 & 9.8 & 5.0 & ----- & 0.35 \\
\hline Opt & 136.6 & 9.8 & 5.0 & 3.2 & 0.35 \\
\hline
\end{tabular}

\subsection{Interfacial Properties Calibration}

To model the homogenized response under off-axis loading, the in-plane and out-of-plane cohesive parameters need to be calibrated first. In Section 7.4.1 the in-plane cohesive parameters 
are calibrated by targeting the experimental transverse response for which specimen failure is due to in-plane fiber/matrix interfacial debonding and matrix cracking. In Section 7.4.3 the outof-plane cohesive parameters are calibrated by targeting the $15^{0}$ off-axis specimen response for which non-linear homogenized out-of-plane shear response is assumed to be due to the interfacial damage along the fiber direction. It is known that the Celion 6000/PMR-15 composite is consolidated at a very high temperature since the glass transition temperature for the resin is $327^{\circ} \mathrm{C}$. As the room temperature is assumed to be $25^{\circ} \mathrm{C}$, cool-down temperature of $302^{\circ} \mathrm{C}$ is used in the analysis before the mechanical loading is applied. And the temperature cool-down effect is investigated in section 7.4.2.

\subsubsection{In-Plane Cohesive Properties}

The uncoupled bilinear cohesive law is employed for in-plane loading and the cohesive parameters are assumed to be the same for normal and tangential directions. Three parameters need to be determined, namely the elastic interfacial stiffness, $k^{0}$, the interfacial strength and the final separation distance $\Delta^{c}$. Figure 7.5 shows one type of CZM-based distribution of interfaces that may experience damage leading to specimen failure under transverse loading. For this distribution, the specimen failure under transverse loading is characterized by fiber/matrix debonding and matrix cracking. To account for these damage and failure modes, CZM-based interfaces are inserted along the fiber/matrix interfaces and inside the PMR-15 matrix.

The principle used to determine the elastic interfacial stiffness, $k^{0}$ is that the value of $k^{0}$ is high enough so that the unit cell with or without interface produces similar response in the elastic stage of the cohesive law. The value of $20 \times 10^{3} \mathrm{MPa} / \mu m$ satisfies this requirement. Figure 7.6 illustrates the homogenized out-of-plane shear response for three different final separation distances and fixed interfacial strength of $61 \mathrm{MPa}$, denoted as Cases 1, 2 and 3 in Table 7.7. In the actual experiment, the specimen experiences ultimate failure when the tensile stress reaches $52 \mathrm{MPa}$, and all three cases show very good agreement with the experimental data before the ultimate failure. Regarding the ultimate failure, the final separation distance $\Delta^{c}$ controls how dramatic the unit cell will fail. As observed in Figure 7.6 when the final separation is smaller, the specimen fails more dramatically. The final separation distance $0.17 \mu \mathrm{m}$ provides a significant loading drop although not complete failure. To model the complete failure, the interface 
distribution in the unit cell needs to be further redesigned to enable complete and continuous separation of the material inside the unit cell.

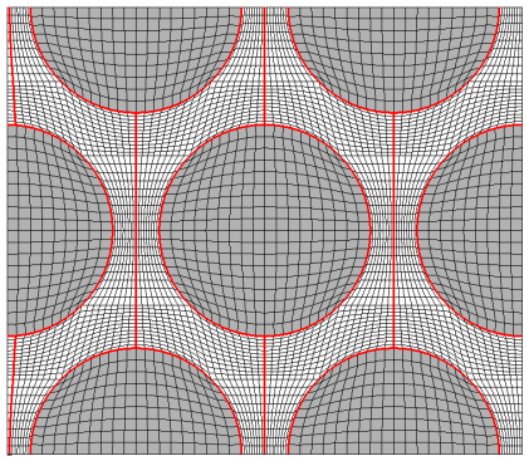

Figure 7.5 Interface distributions inside a hexagonal unit cell.

Table 7.7 Interfacial parameters used for transverse loading.

\begin{tabular}{|c|c|l|c|c|c|}
\hline Case type & $\begin{array}{c}K_{n}^{0}=K_{t}^{0}=K_{o p}^{0} \\
(\mathrm{MPa} / \mu m)\end{array}$ & \multicolumn{1}{|c|}{$\left(\mathrm{J} / \mathrm{m}^{2}\right)$} & $\begin{array}{c}\text { Interfacial strength } \\
(\mathrm{MPa})\end{array}$ & $\begin{array}{c}\Delta_{n}^{0}=\Delta_{t}^{0}=\Delta_{o p}^{0} \\
(\mu m)\end{array}$ & $\begin{array}{c}\Delta_{n}^{c}=\Delta_{t}^{c}=\Delta_{o p}^{c} \\
(\mu m)\end{array}$ \\
\hline Case 1 & $20 \times 10^{3}$ & 15.25 & 61 & $3.05 \times 10^{-3}$ & 0.5 \\
\hline Case 2 & $20 \times 10^{3}$ & 7.6 & 61 & $3.05 \times 10^{-3}$ & 0.25 \\
\hline Case 3 & $20 \times 10^{3}$ & 5.2 & 61 & $3.05 \times 10^{-3}$ & 0.17 \\
\hline
\end{tabular}

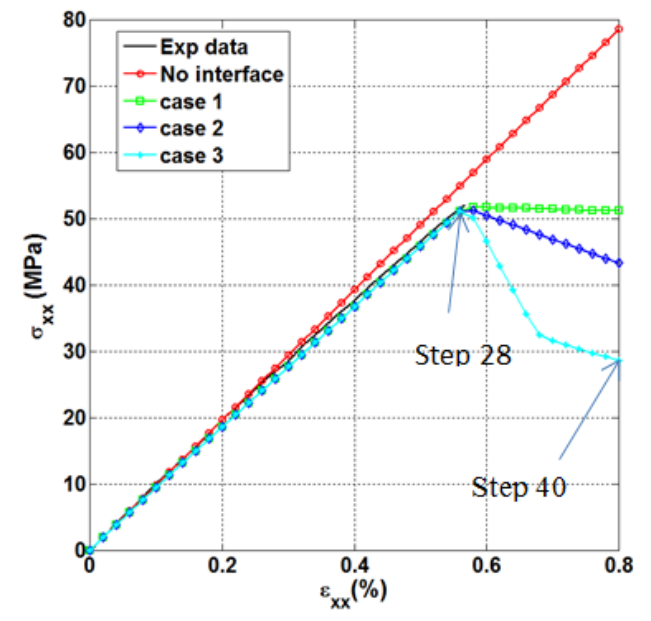

Figure 7.6 Homogenized responses with interfacial strength $61 \mathrm{MPa}$ and different final separation distance.

Figure 7.7 illustrates the stress distributions at the step when damage initiates and at the final loading step. It is no surprise that the stress distributions in all three cases are initially the same before and at damage initiation step, step 28, since they are the same problem when the interfaces are in the elastic stage. Figure 7.6 shows that the drop in load capacity increases with decreasing final separation distance. This is directly reflected in the $\sigma_{22}$ stress distributions of 
Figure 7.7 at step 40, the final loading step, where in general the stress distribution in both matrix and fiber phases for Case 3 is substantially lower than the other two cases.

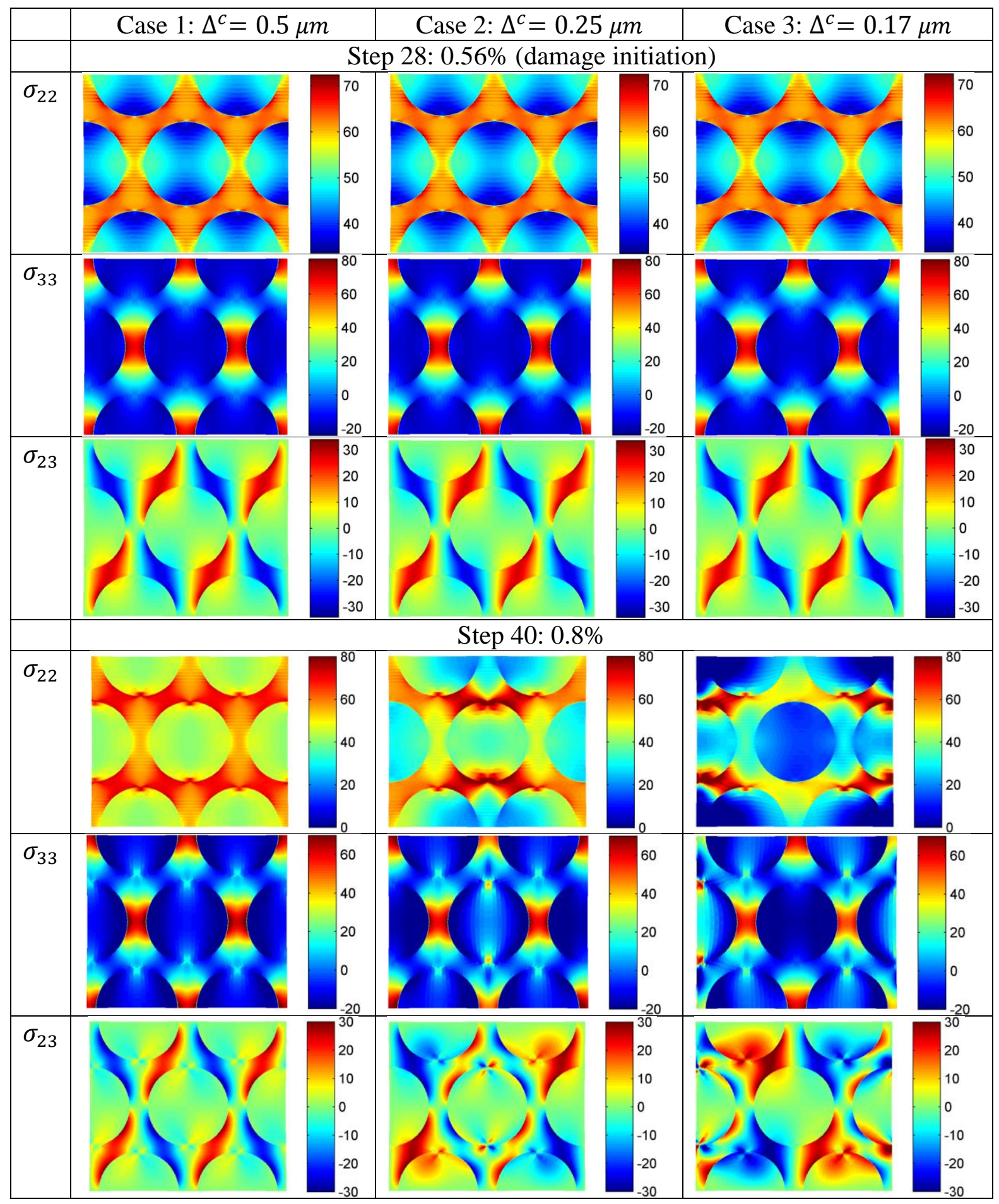

Figure 7.7 Stress distributions at damage initiation step and final loading step. 


\subsubsection{Effect of Temperature Cool-Down}

In the unit cell analysis of the preceding section, cool-down temperature of $302^{\circ} \mathrm{C}$ was used to estimate the residual stresses before characterizing the interface parameters. Because of the mismatch between fiber and matrix thermo-elastic moduli, substantial residual stresses are introduced into the material system after the fabrication temperature cool-down. The residual stresses may potentially affect the response of the material. In this section, the impact of residual stresses on the interfacial property determination is demonstrated.

Figure 7.8 shows the homogenized response of the unit cell under transverse loading with and without consideration of temperature cool-down when the cohesive parameters of Case 3 listed in Table 7.7 are used. As observed in the figure, if temperature cool-down is not considered, the material will start failing at $44 \mathrm{MPa}$, which is lower than the test data and the prediction with temperature cool-down, 52MPa. Also, the failure process for the case without temperature cool-down is less catastrophic than the case with temperature cool-down. In contrast, experiment indicates the material system fails catastrophically during the test. Therefore, to correctly capture the experimental response, the temperature cool-down effect needs to be taken into account in characterizing the interface parameters.

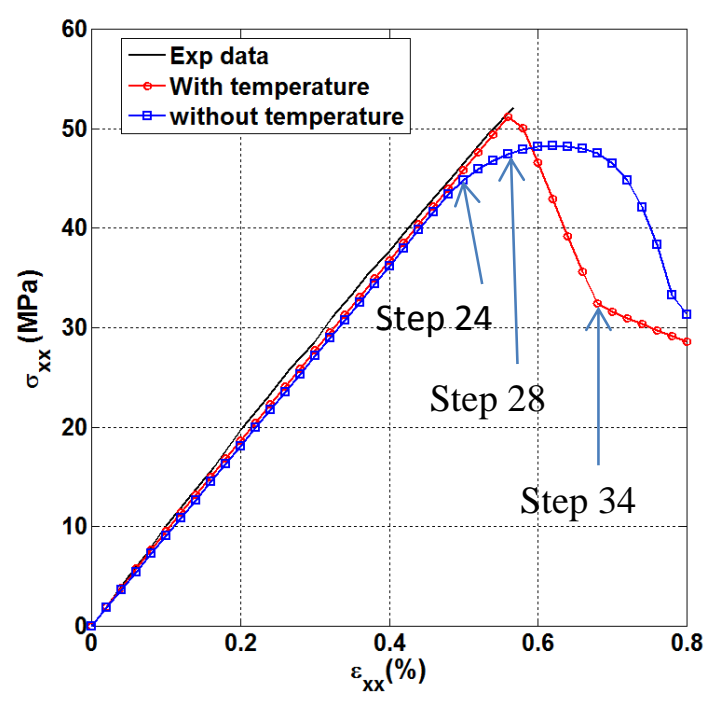

Figure 7.8 Homogenized response under transverse loading with and without consideration of temperature cool-down. 
Figure 7.9 shows the stresses developed inside the unit cell due to temperature cooldown, which are substantial relative to the interfacial strength of $61 \mathrm{MPa}$ calibrated by targeting the experimental response under transverse loading. Figures 7.10-7.11 illustrate the stress distributions at steps 24 and 34 of the unit cell with and without consideration of temperature cool-down. At step 24, the interface is about to reach damage initiation when temperature cooldown is not considered whereas this occurs at step 28 when temperature cool-down is considered. The $\sigma_{22}$ stress distribution in the unit cell with consideration of temperature cooldown is much more uniform than the distribution in the unit cell without consideration of temperature cool-down. Because of this, when $\sigma_{22}$ attains interfacial strength in the case with temperature cool-down, interface damage will develop much quicker than the case without temperature cool-down. As illustrated in Figure 7.11, $\sigma_{22}$ in the unit cell with temperature cooldown is substantially lower than the stress in the unit cell without temperature cool-down.

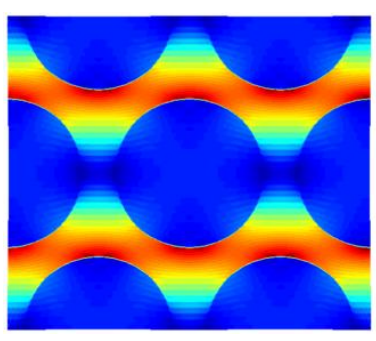

(a) $\sigma_{22}$

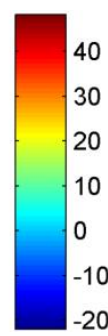

$-20$

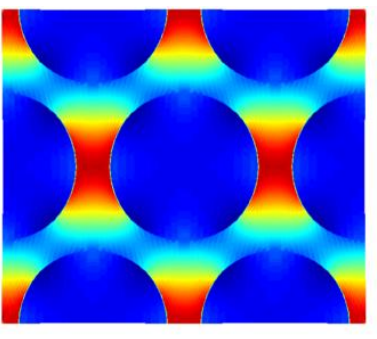

(b) $\sigma_{22}$
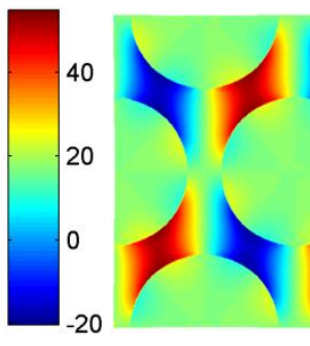

(c) $\sigma_{23}$

Figure 7.9 Full-field stress distributions after temperature cool-down of $302^{\circ} \mathrm{C}$. 


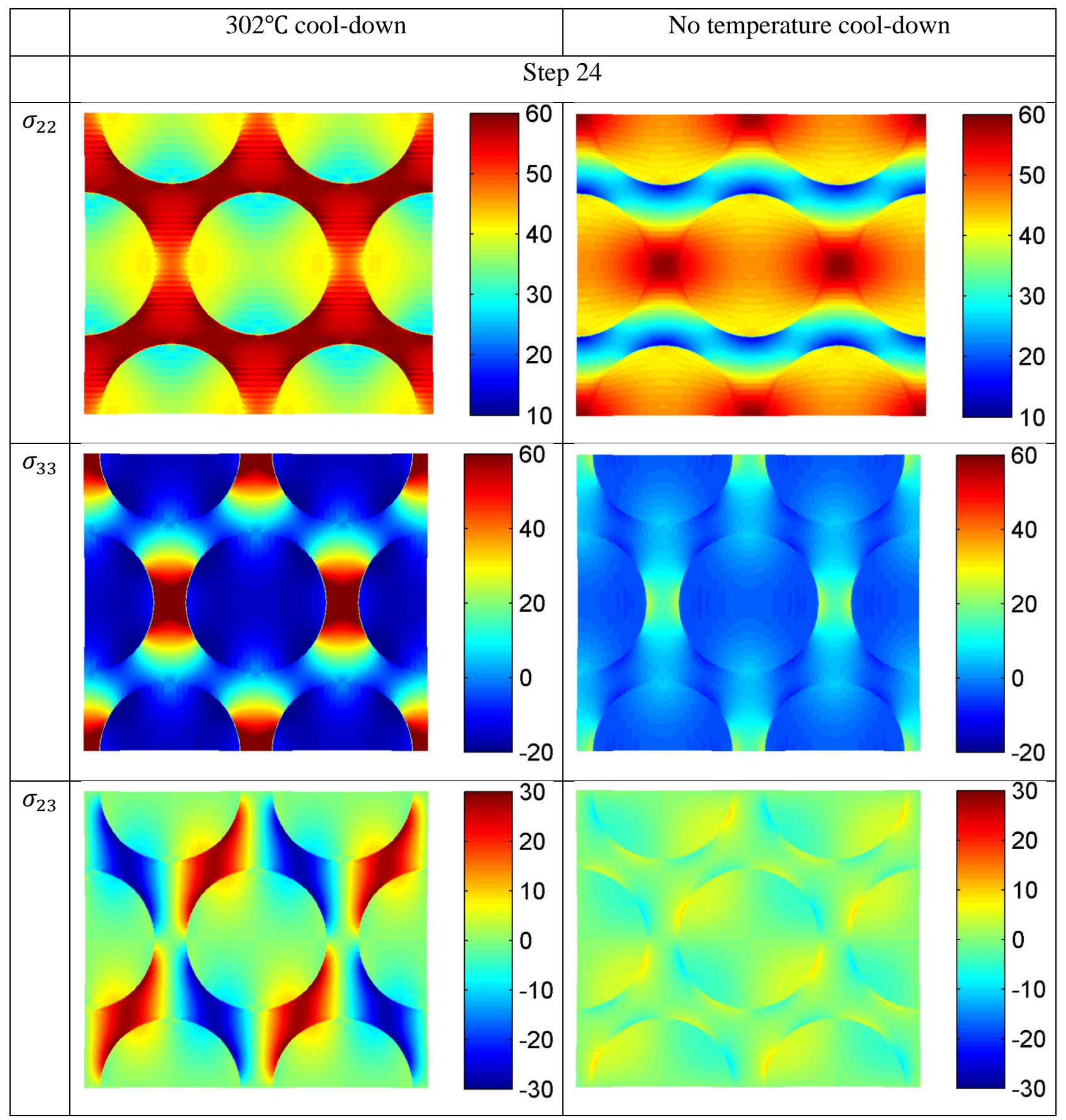

Figure 7.10 Full-field stress distributions at step 24 with and without temperature cool-down. 


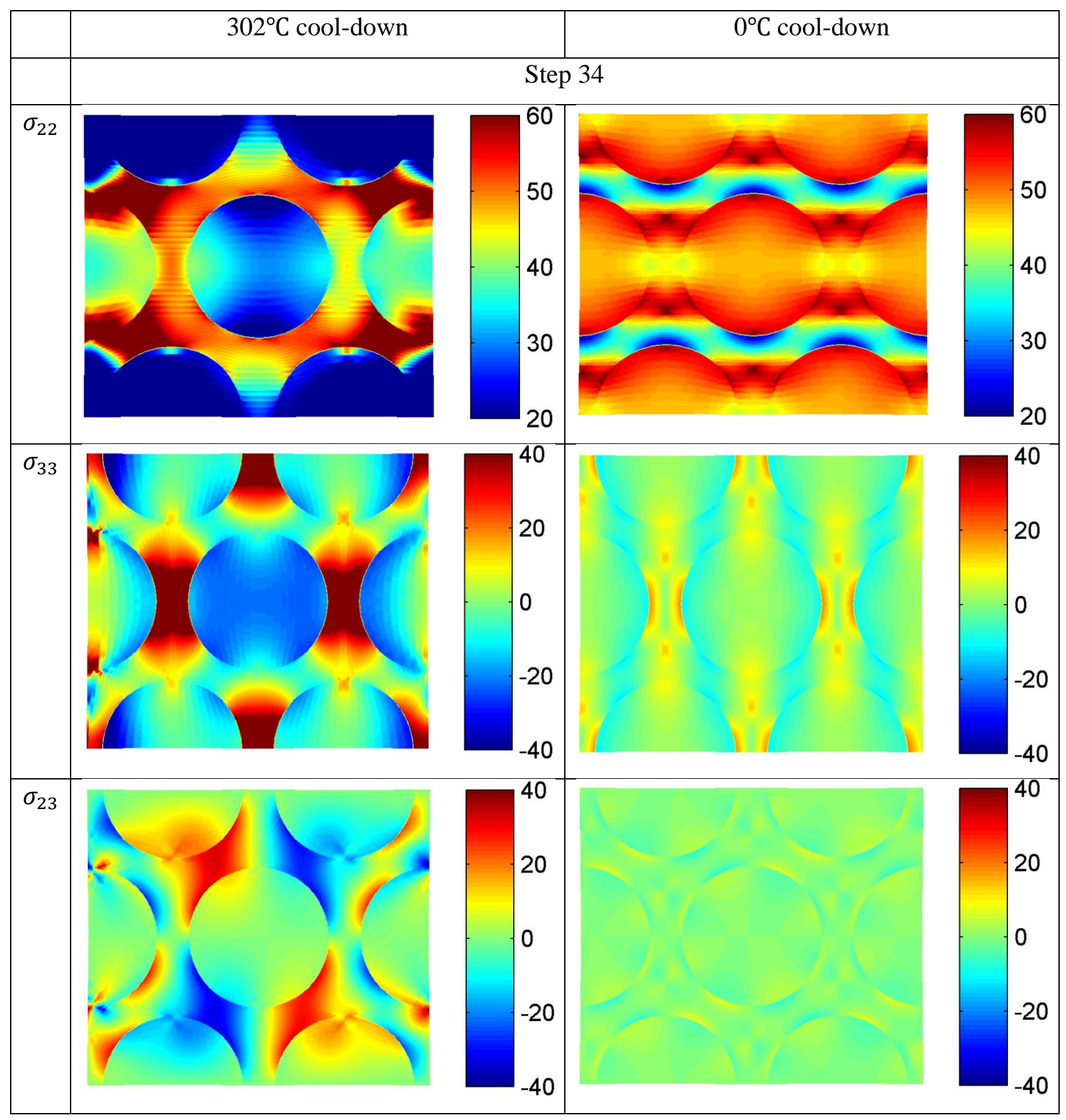

Figure 7.11 Full-field stress distributions at step 34 with and without temperature cool-down. 


\subsubsection{Out-of-Plane Cohesive Properties}

Similarly, there are three parameters that need to be determined for the out-of-plane cohesive law, namely the elastic interfacial stiffness, $k_{o p}^{0}$, the interfacial strength and the final separation distance $\Delta^{c}$. The interfacial stiffness can be relatively easily determined by choosing a high value which produces similar response for the unit cell with or without interfaces experiencing damage. The value of $20 \times 10^{3} \mathrm{MPa} / \mu \mathrm{m}$ satisfies this requirement. The other two out-of-plane cohesive parameters, interfacial strength and final separation are calibrated by targeting the $15^{\circ} \mathrm{C}$ off-axis specimen loading case for which the non-linear homogenized out-of-plane shear response is due to the interfacial damage along the fiber direction.

Figure 7.12 shows two types of damage-evolving interface distributions in a hexagonal unit cell. In Case 1 cohesive interfaces are inserted around all fiber/matrix interfaces, while in Case 2 no interfaces are inserted around the middle row of fibers. Figure 7.13 illustrates the homogenized out-of-plane shear response for the two types of cohesive interface distributions shown in Figure 7.12 with the parameters given in Table 7.8. As observed in Figure 7.13, the simulated homogenized response exhibits excellent agreement with the experimental data with the cohesive interface distribution of Case 2 when the interfacial strength is $30 \mathrm{MPa}$ and the final separation distance is $0.5 \mathrm{MPa} / \mu \mathrm{m}$. The reason why the response of Case 2 is stiffer than that Case 1 in the non-linear stage is because the central part of the unit cell can continuously sustain loading. This point will be illustrated in the sequel by examining the stress distributions.

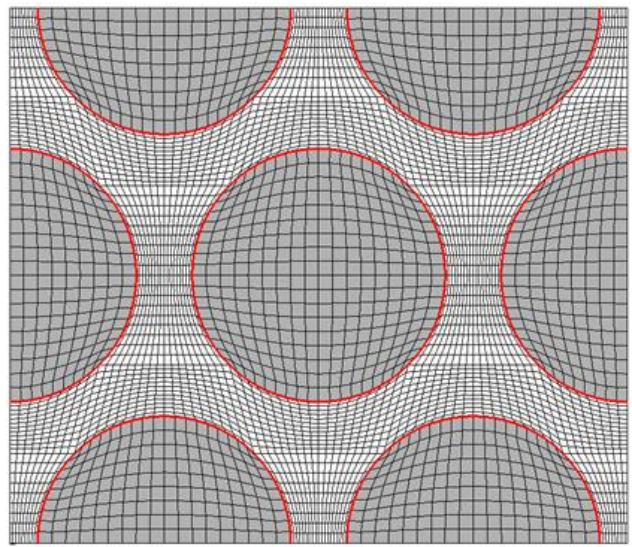

Case 1

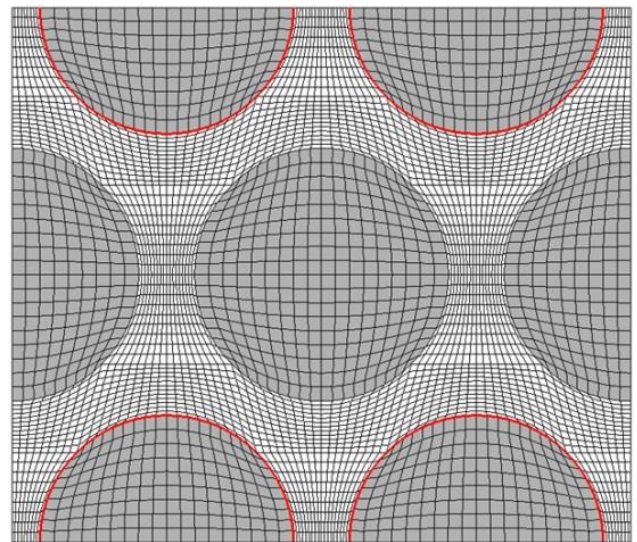

Case 2

Figure 7.12 Interface distributions inside a hexagonal unit cell. 
Table 7.8 Interfacial parameters for out-of-plane shear response.

\begin{tabular}{|c|c|c|c|c|c|}
\hline Case type & $K_{o p}^{0}(\mathrm{MPa} / \mu m)$ & $\mathrm{G}\left(\mathrm{J} / \mathrm{m}^{2}\right)$ & $\begin{array}{c}\text { Interfacial strength } \\
(\mathrm{MPa})\end{array}$ & $\Delta_{o p}^{0}(\mu m)$ & $\Delta_{o p}^{c}(\mu m)$ \\
\hline Case 1 & $20 \times 10^{3}$ & 7.5 & 30 & $1.5 \times 10^{-3}$ & 0.50 \\
\hline
\end{tabular}

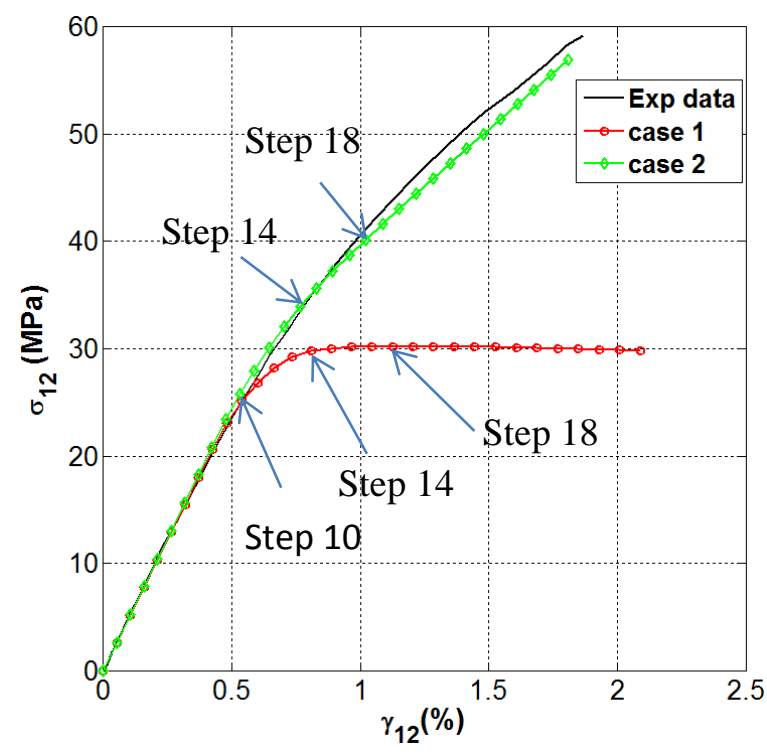

Figure 7.13 Homogenized shear response of $15^{\circ} \mathrm{C}$ off-axis specimen for different interface distributions.

Figures 7.14-7.16 illustrate the stress distributions for the above two cases at steps 10, 14 and 18. These distributions include the transverse normal and shear stresses $\sigma_{22}$ and $\sigma_{23}$ due to the macroscopic stress $\bar{\sigma}_{22}$ in the principal material coordinate system, and the axial shear stresses $\bar{\sigma}_{12}$ and $\bar{\sigma}_{13}$ due to the macroscopic stress $\bar{\sigma}_{12}$. The stress distributions $\sigma_{22}$ and $\sigma_{23}$ in both cases at the selected steps are similar because they are not sufficiently high to reach the interfacial normal and tangential strengths of 61MPa calibrated in Section 7.4.1 under transverse loading. It is the axial shear stress $\sigma_{12}$ which is dominant for this off-axis configuration and which initiates interfacial degradation leading to the homogenized response dramatically sensitive to the placement of interfaces that experience damage shown in Figure 7.13. Specifically, after damage initiation at step 10, the axial shear stress in Case 1 is substantially lower than its counterpart in Case 2 because in the former case all fiber/matrix interfaces experience degradation, while in Case 2 the central region of the unit cell continuously supports loading. This type of interface configuration targets the experimental data very well, illustrating that it is sufficient for only some of the interfaces to contribute to the nonlinear response. To 
model complete failure, more interfaces with different interfacial strengths need to be inserted into the unit cell, so that the material can completely fail when the ultimate strength is reached. The study of complete failure is out of the scope of current thesis.

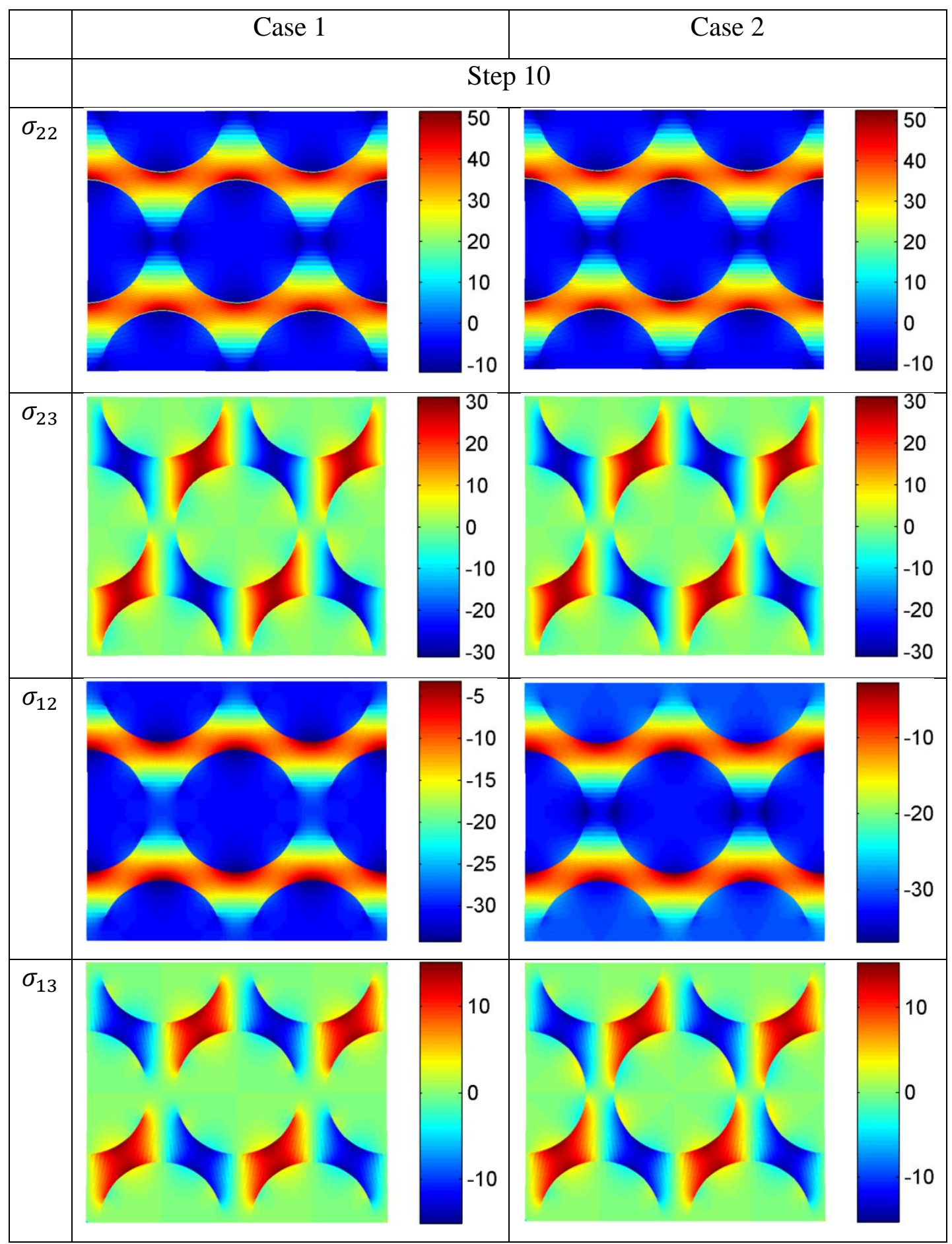

Figure 7.14 Stress distributions for two types of interface configuration at step 10. 


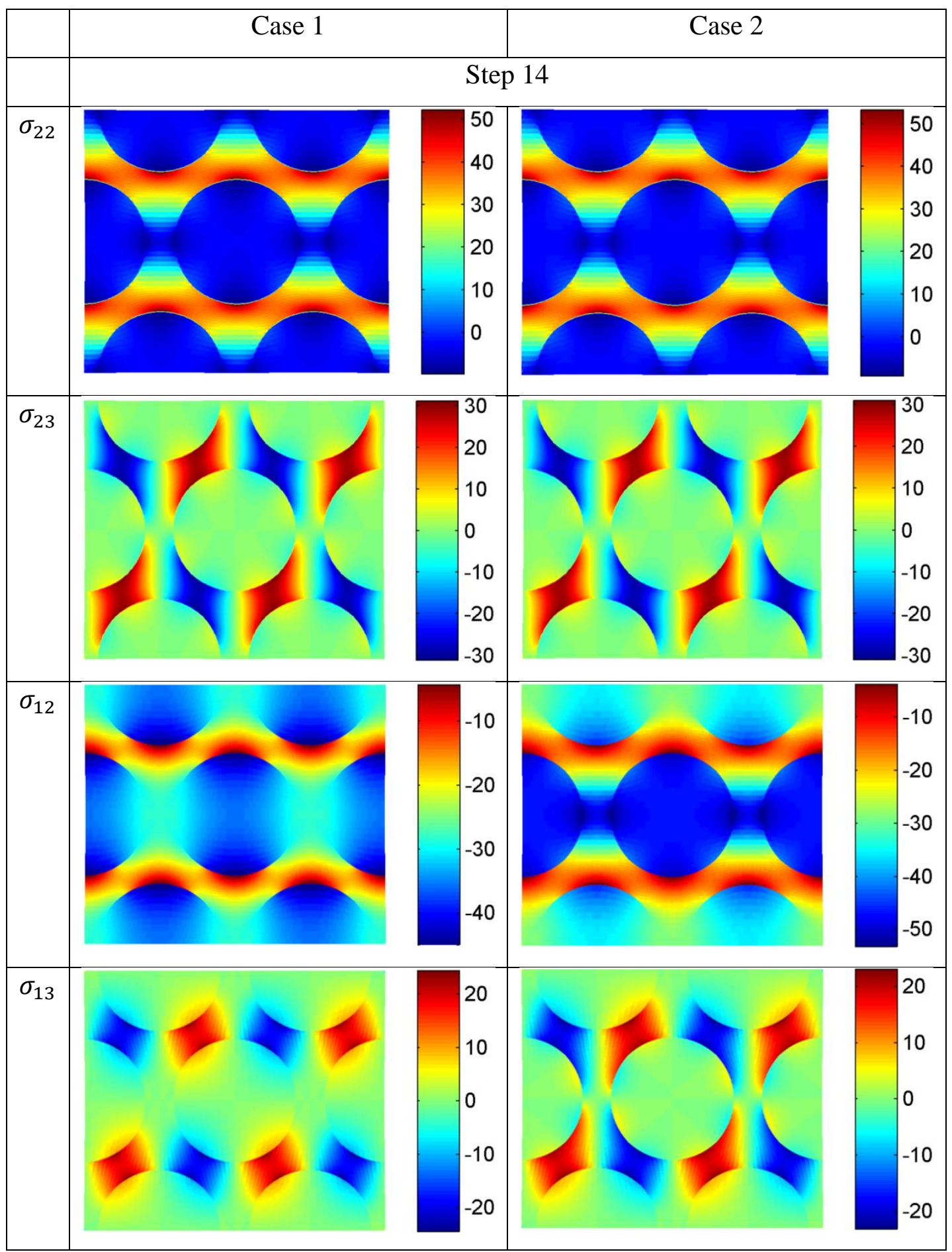

Figure 7.15 Stress distributions for two types of interface configuration at step 14. 


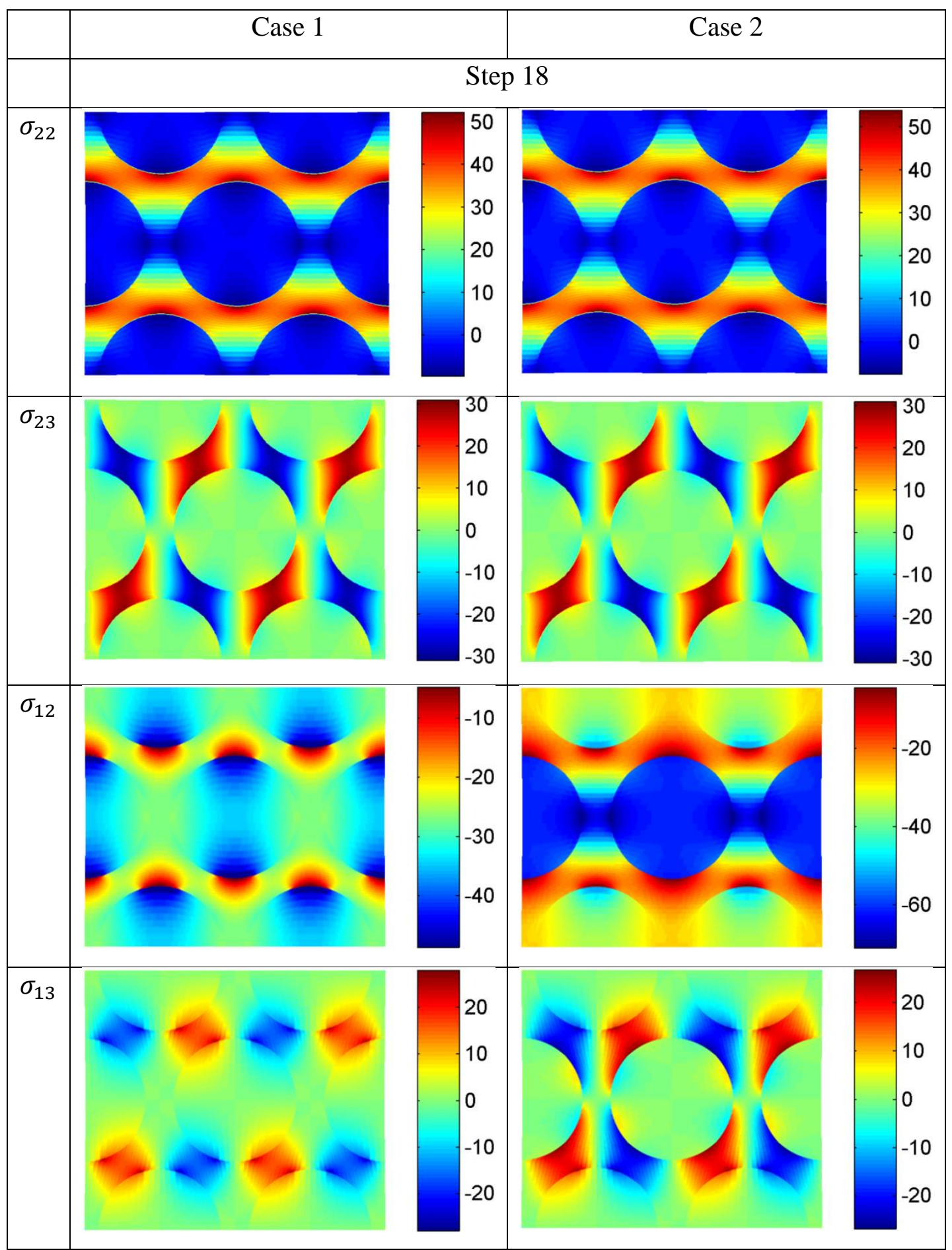

Figure 7.16 Stress distributions for two types of interface configuration at step 18. 


\subsection{Numerical Results}

\subsubsection{Monotonic Response in Principal Material Coordinates}

It is known that for the considered unidirectional composite substantial nonlinearity is observed in the shear response of off-axis specimens in the principal material coordinate system. In contrast, the nonlinear transverse response is minimal and the axial response is elastic. Hence the nonlinear shear response controls the observed nonlinear response in the laminate coordinates system of the tested graphite/polyimide off-axis specimens. In Section 7.4.1, the in-plane cohesive properties have been calibrated using the transverse tension results of $90^{\circ}$ specimens, and in Section 7.4.3 the out-of-plane cohesive properties and the corresponding interface configuration have been identified by targeting the test data obtained from $15^{0}$ off-axis specimens. In this section the shear responses of specimens with different off-axis angles are predicted based on the previously calibrated in-plane and out-of-plane cohesive parameters and the identified interface distribution.

Figure 7.17 illustrates the comparison between FVDAM's predictions and experimental data for $10^{\circ} \mathrm{C}$ and $45^{\circ} \mathrm{C}$ off-axis specimens where very good agreement is observed between theoretical results and experiment, especially for the $45^{\circ}$ off-axis loading case. Figure 7.18 summarizes the experimental and theoretical comparison for different off-axis loading angles. As expected, the elastic response of the tested specimens does not change with off-axis orientation as there is no coupling between normal and shear stress and strain components for transversely isotropic materials. The theoretical predictions confirm this, exhibiting excellent correlation with the experiment.

In the nonlinear region, however, small but consistent differences are observed in the experimental results among the different off-axis orientations. These differences indicate the presence of interaction between the three stress components $\sigma_{11}, \sigma_{22}$ and $\sigma_{12}$ in the principal material coordinate system of the tested specimens. In the current version of FVDAM this interaction is absent with the result that the same nonlinear shear response is predicted in the principal material coordinate system regardless of the off-axis loading angle. This is a direct result of the absence of coupling between the out-of-plane and in-plane cohesive laws. Nonetheless, the differences amongst the shear responses for different off-axis loading angles are 
limited, with the FVDAM predictions bounded by the experimental data, thereby capturing the average nonlinear shear response of the tested specimens.
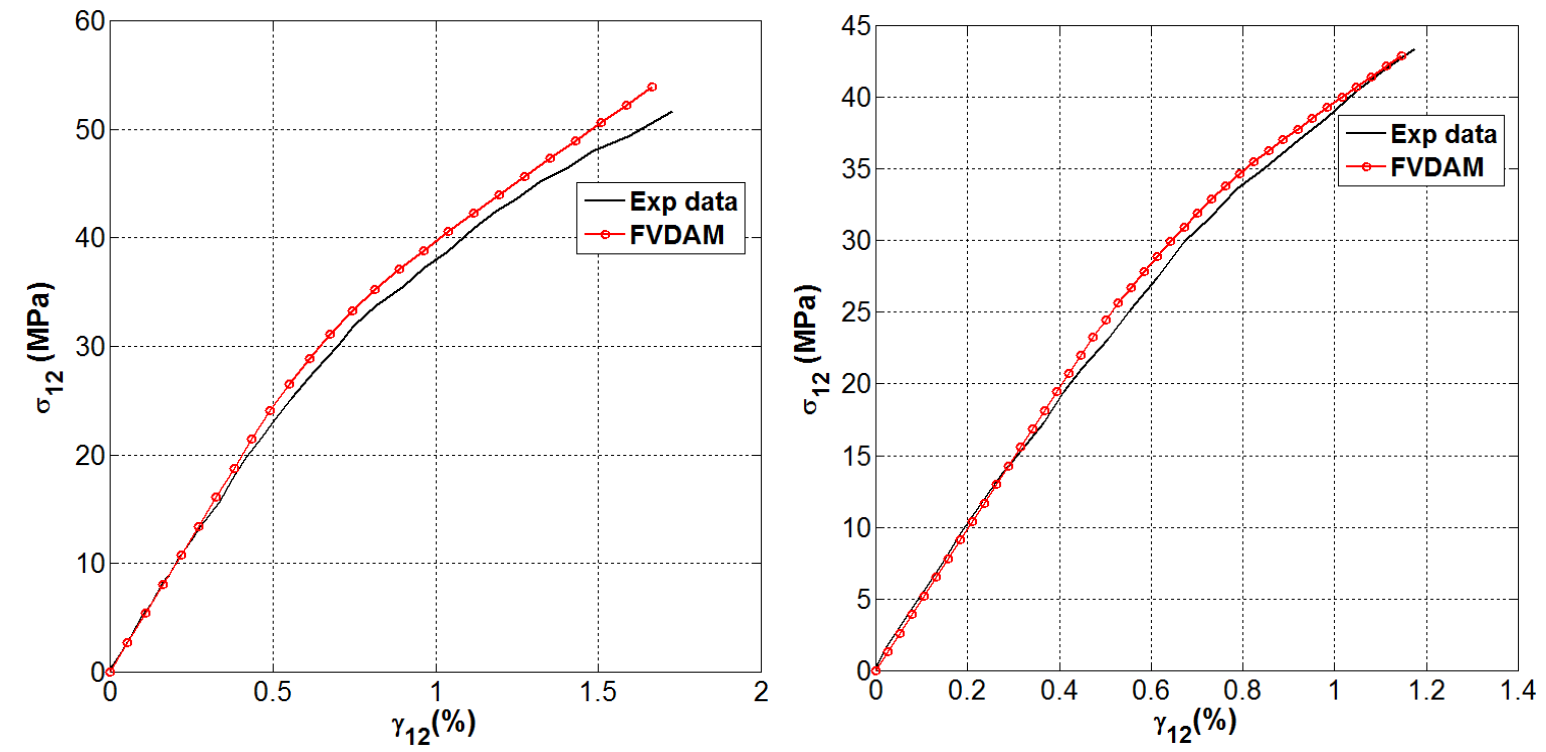

Figure 7.17 Homogenized shear responses under $10^{\circ}$ (Left) and $45^{0}$ (Right) off-axis loading.

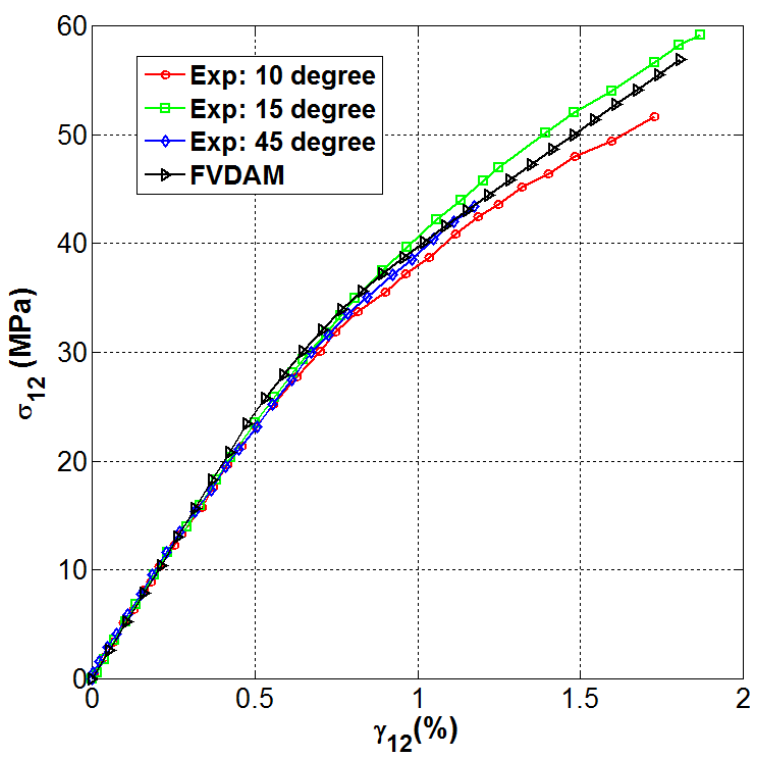

Figure 7.18 Shear responses under different off-axis loading angles and FVDAM's prediction.

\subsubsection{Monotonic Response in the Laminate Coordinate System}

In this section, responses of the tested off-axis graphite/polyimide specimens are compared with the FVDAM predictions in the laminate or global coordinate system that is the coordinate system 
aligned with the specimens' axes. This is a critical test of the FVDAM predictive capability as the initial elastic response rapidly varies with the off-axis angle, decreasing from a large value for the $0^{0}$ specimens to a small value for the $90^{\circ}$ specimens.

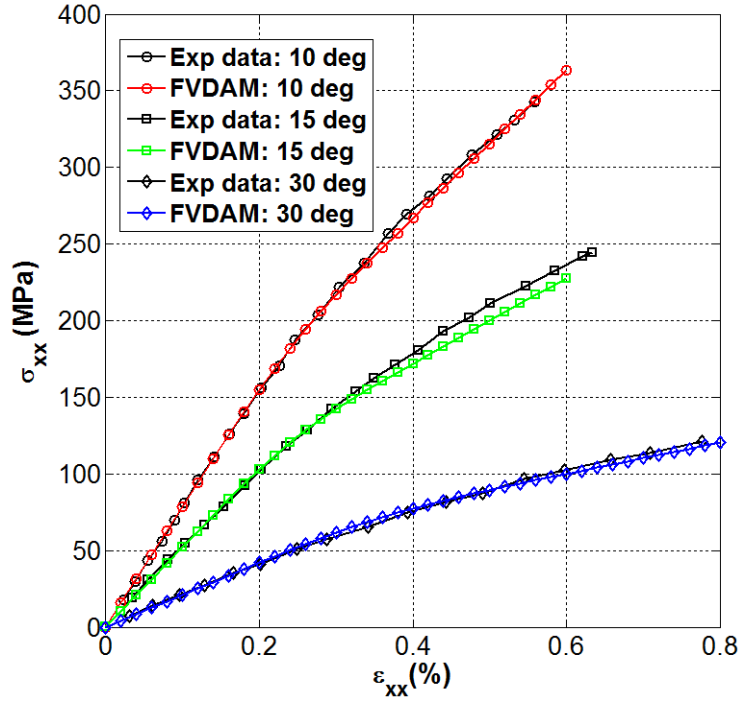

(a)

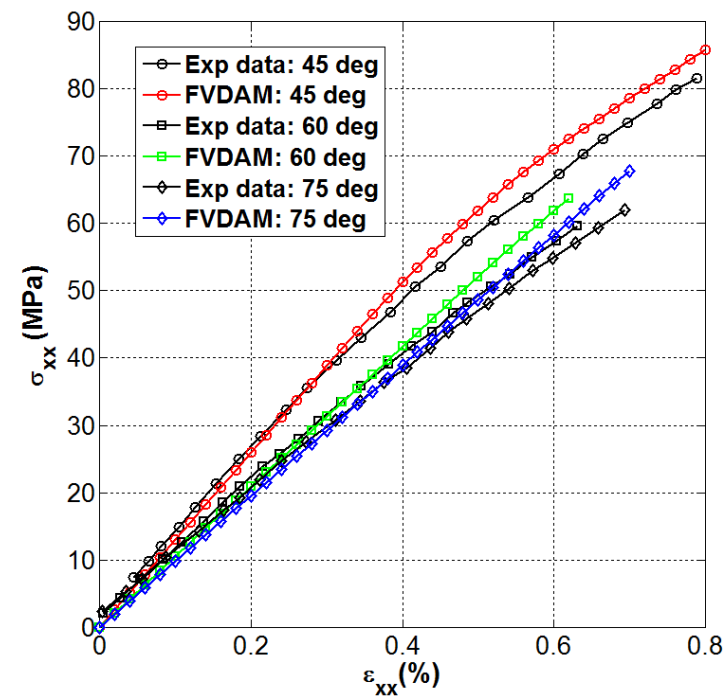

(b)

Figure 7.19. Comparison between experimental data and FVDAM's predictions for laminate response under different off-axis loading axis.

The comparison between experimental data and FVDAM predictions is illustrated in Figure 7.19 for six off-axis configurations ranging from $10^{0}$ to $75^{\circ}$. Figure 7.19 (a) shows the comparison in the small off-axis range where the change in the elastic response is most rapid. The nonlinear response is also more pronounced in this off-axis range because of the high out-ofplane shear stress component $\sigma_{12}$ which induces interfacial damage. Excellent agreement of the FVDAM predictions with experimental data is observed in this range, suggesting that the effect of stress interaction observed in the shear response in the principal material coordinate system is relatively small in this off-axis range. Figure 7.19(b) shows the corresponding comparison for off-axis loading with bigger angles where substantially smaller nonlinearity is observed as the transverse stress component $\sigma_{22}$ becomes dominant for angles greater than $45^{\circ}$. In this case good agreement is also observed, with FVDAM predictions correctly predicting smaller nonlinearity with increasing off-axis angles observed in the experimental data. 


\subsection{Summary}

In this chapter, the off-axis response of graphite/polyimide specimens was simulated based on the assumption that the observed nonlinearity is largely due to the fiber/matrix interfacial damage arising from the significant out-of-plane shear stress components $\sigma_{12}$ in the principal material coordinate system. The simulated responses were shown to exhibit good agreement with the experimentally measured shear responses of the tested off-axis specimens in the principal material coordinate system, as well as with the normal stress responses in the laminate coordinate system.

The experimental-theoretical correlation procedure demonstrated the full range of the developed CZM-based FVDAM damage-simulation capability. Specifically, the simulation analysis required accurate determination of certain fiber and matrix thermo-elastic moduli as well as the in-plane and out-of-plane cohesive parameters. The fiber and matrix phase properties were systematically determined by targeting the homogenized moduli of the tested specimens via the Particle Swarm Optimization approach. The in-plane and out-of-plane cohesive parameters were obtained by targeting the experimental responses of tested $90^{\circ}$ and $15^{\circ}$ off-axis specimens, accounting for the temperature cool-down effects which produce higher damage initiation loads and more catastrophic failure modes. The adopted procedure produced theoretical predictions with very good correlation with experiment, supporting the original hypothesis that the observed nonlinearity in this brittle-brittle composite system may be explained by shear-dominated interfacial damage evolution. 


\section{Chapter 8}

\section{Accomplishments, Summary and}

\section{Conclusions}

This work has produced a new approach for simulating damage evolution in heterogeneous materials characterized by complex microstructures which possess periodicity in one plane and which do not vary along the orthogonal direction. Unidirectional composites characterized by periodically distributed reinforcement in the orthogonal plane belong in this category. These unidirectional composites are the building blocks for composites laminates used across disciplinary boundaries from aerospace and civil engineering structural components to electronic devices such as circuit boards. Hence the characterization of the inelastic response of these building blocks due to damage is the first step in simulating the structural response of laminated constructs. The developed theory may also be used to simulate damage at the meso-level where damage evolution does not vary along one direction. An example of this includes damage evolution in symmetric bidirectional laminated plates where damage occurs in the plies with fibers oriented orthogonally to the applied load.

The developed approach combines the finite-volume direct averaging micromechanics (FVDAM) theory with cohesive zone model capability, incorporated with the aid of displacement discontinuity functions. This framework essentially separates the system of governing equations that control the response of a periodic composite undergoing damage evolution into primary and auxiliary systems, with the primary system solved just once. The formulation eliminates the necessity to re-assemble the global system of equations during damage evolution, determined by iteratively solving the auxiliary system of equations which governs damage progression. The added benefit is that the propagation of stationary cracks, and calculation of concomitant energy release rates, is also treated within the same framework upon modifying the auxiliary system of equations in a straightforward manner. Another important feature of the framework is the ease with which compressive stress normal to the interface undergoing damage is treated. In contrast with typical finite-element approaches which rely on 
artificially large interfacial stiffness when the normal stress becomes compressive, in the developed approach the governing equations for a damaged interface in the auxiliary system of equations are simply eliminated. This circumvents the problem of large compressive normal stresses arising at the interface undergoing damage when stress reversal occurs. Overall, the developed homogenized theory provides an efficient and convenient tool that may be used to characterize the effect of damage on the elastic and inelastic response of periodic materials with oriented reinforcement and damage evolution at the micro-scale (constituent phase) or the mesoscale (lamina) levels.

The novelty arises from the finite-volume framework that is just beginning to be employed to investigate damage evolution in periodic materials within the homogenization theory, and the cohesive zone model capability incorporated via the displacement discontinuity functions. This general formulation facilitates the incorporation of any interfacial tractiondisplacement discontinuity constitutive law, including the CZM capability illustrated in this work. The semi-analytical framework with derived closed-form expressions for the local stiffness matrix elements that govern the individual subvolume response facilitated incorporation of the developed theory into the Particle Swarm Optimization algorithm, producing a powerful design tool for identifying optimal material architectures as well as parameters that are not easily measured experimentally, such as the CZM parameters or the elastic moduli of graphite fibers.

The CZM-based FVDAM theory has been verified upon comparison with exact elasticity solutions in the elastic stage of damage evolution, and experimental data and finite-element simulations at both the microlevel and macrolevel in the nonlinear stage of damage evolution. The computational tool has also been employed to investigate the response of composite materials undergoing damage and the underpinning mechanisms. The major accomplishments are described below.

- Validation with the experimental data on SiC/Ti unidirectional composites under transverse loading correctly captured the evolution of fiber/matrix interfacial debonding and subsequent arrest of the debond progression due to the development of compressive stress normal to the interface. The importance of residual stresses has been revealed, demonstrating that correct simulation of fiber/matrix debonding was not possible without these stresses. 
- Comparison with finite-element simulation of evolving fiber/matrix interfacial damage in $\mathrm{SiC} / \mathrm{Ti}$ unidirectional composites under transverse loading did not reveal any differences in the entire loading range, including elastic response, damage initiation, progression and separation. While appropriate adjustment of the interfacial stiffness under compressive normal stresses was required to obtain accurate and converged results with the employed commercial finite-element code, this problem is not an issue with the developed CZM-based FVDAM theory.

- Simulation of evolving damage on the fly in polymeric matrix cross-ply laminates, caused by progressive cracking of the inner $90^{\circ}$ plies and interfacial debonding between adjacent plies, produced good correlation with experimental data, revealing previously undocumented and dramatic effect of damage on the transverse Poisson's response. This effect was an excellent indicator of both damage initiation and evolution in contrast with the axial response. Moreover, several features observed in the homogenized transverse response could be correlated with the underpinning mechanisms such as instantaneous and progressive cracking and delamination. Another significant result was the demonstration of a damage mode switch from transverse cracking of the $90^{\circ}$ plies to delamination between the $0^{\circ}$ and $90^{\circ}$ plies. While postulated by a number of researchers, this phenomenon was demonstrated on the fly for the first time in the present work.

- The simulation of damage in graphite/polyimide unidirectional off-axis specimens based on the hypothesis of shear-dominated fiber/matrix interfacial degradation mimicked well the nonlinear homogenized response in both the principal material and laminate coordinate systems. This study revealed for the first time that the off-axis dependent nonlinearity in this material system comprised of linearly elastic fiber and matrix phases could be captured using a damage evolution model rather than a plasticity, viscoelasticity or viscoplasticity approach for the matrix phase.

This investigation has demonstrated the feasibility of employing a finite-volume based homogenization theory coupled with an efficient implementation of a cohesive zone model to simulate on the fly damage evolution in periodic materials characterized by oriented microstructures and damage modes. While more work remains to be done to enhance the theory's predictive capability for simulating damage in the considered class of microstructures, the generated results suggest that the theory's extension to enable damage simulation in three- 
dimensional microstructures will be successful. The present version will benefit from the incorporation of inelastic effects at the constituent phase level, such as plasticity, viscoplasticity and viscoelasticity theories for the matrix phase, as well as the implementation of an algorithm to track damage evolution in arbitrary directions. 


\section{Reference}

Aboudi J, Pindera M-J and Arnold SM, 1999. Higher-order theory for functionally graded materials, Composites Part B: Engineering, 30 (8): 777-832.

Aboudi J, Pindera M-J and Arnold SM, 2001. Linear thermoelastic higher-order theory for periodic multiphase materials, Journal of Applied Mechanics, 68 (5): 697-707.

Aboudi J, Pindera M-J and Arnold SM, 2002. High-fidelity generalized method of cells for inelastic periodic multiphase materials. NASA Technical Memorandum 2002-211469, NASAGlenn Research Center, Cleveland, OH. March 2002.

Achenbach JD, 1975. A theory of elasticity with microstructure for directionally reinforced composites. Springer-Verlag, New York.

Akula VMK and Garnich MR, 2012. Effective ply and constituent elastic properties for cracked laminates, Composites Part B: Engineering, 43: 2143-2151.

Bader MG, Bailey JE, Curtis PT and Parvizi A, 1979. The mechanisms of initiation of development of damage in multi-axial fiber-reinforced plastics laminates, International Conference on Mechanical behaviour of materials, Carrbridge, 227.

Bailey C and Cross M, 1995. A finite volume procedure to solve elastic solid mechanics problems in three dimensions on an unstructured mesh, International Journal for Numerical Methods in Engineering, 38: 1757-1776.

Bailey JE, Curtis PT and Parvizi A, 1979. On the transverse cracking and longitudinal splitting behavior of glass and carbon fibre reinforced epoxy cross-ply laminates and the effect of Poisson and thermally generated strain, Proceedings of the Royal Society A, 366: 599-623.

Banea MD and da Silva LFM, 2009. Adhesively bonded joints in composite materials: An overview, Proceedings of the Institution of Mechanical Engineers, Part L: Journal of Materials: Design and Applications, 223:1-18.

Banks-Sills L and Einav O,1987. On singular, nine-noded, distorted, isoparametric elements in linear elastic fracture mechanics, Computers \& Structures, 25(3): 445-449.

Banks-Sills L and Sherman D, 1989. On quarter-point three-dimensional finite elements in linear elastic fracture mechanics, International Journal of Fracture, 41(3): 177-196.

Banks-Sills L, 1991. Application of the finite element method to linear elastic fracture mechanics, Applied Mechanics Reviews, 44(10): 447-461. 
Banks-Sills L, 2010. Update: application of the finite element method to linear elastic fracture mechanics, Applied Mechanics Reviews, 63(2): 020803(1-17).

Banks-Sills L, Hershkovitz I, Wawrzynek PA, Eliasi R, and Ingraffea AR, 2005. Methods for calculating stress intensity factors in anisotropic materials: Part $\mathrm{I}-\mathrm{z}=0$ is a symmetric plane, Engineering Fracture Mechanics, 72: 2328-2358.

Bansal Y and Pindera M-J, 2003. Efficient reformulation of the thermoelastic higher-order theory for fgms, Journal of Thermal Stresses, 26(11-12): 1055-1092.

Bansal Y and Pindera M-J, 2005. A second look at the higher-order theory for periodic multiphase materials, Journal of Applied Mechanics, 72(2): 177-195. See also: NASA CR2004213043.

Bansal Y and Pindera M-J, 2006. Finite-volume direct averaging micromechanics of heterogeneous materials with elastic-plastic phases, International Journal of Plasticity, 22 (5): 775-825.

Barbero EJ and Cosso FA, 2014. Determination of material parameters for discrete damage mechanics analysis of carbon-epoxy laminates, Composite Part B: Engineering, 56: 638-646.

Barenblatt GI, 1959. The formation of equilibrium cracks during brittle fracture. General ideas and hypothesis. Axially-symmetric cracks, Prikl. Mat. Mekh., 23(3): 434-444.

Barenblatt GI, 1962. Mathematical theory of equilibrium cracks in brittle fracture (Advances in Applied Mechanics, Vol. VII), H. L. Dryden, and T. von Karman, eds., Academic, New York, pp. $55-125$.

Barsoum RS, 1974. Application of quadratic isoparametric finite elements in linear fracture mechanics, International Journal of Fracture, 10(4): 603-605.

Belytschko T and Black T, 1999. Elastic crack growth in finite elements with minimal remeshing, International Journal for Numerical Methods in Engineering, 45: 601-620.

Belytschko T, Gracie R, Ventura G, 2009. A review of extended/generalized finite element methods for material modeling, Modelling and Simulation in Materials Science and Engineering, 17: 043001(1-24).

Belytschko T, Liu WK, Moran B, 2000. Nonlinear finite elements for continua and structures, Wiley: Chichester.

Bensoussan A, Lions J-L, and Papanicolaou G, 1978. Asymptotic analysis for periodic structures, North Holland, Amsterdam, Netherlands. 
Berezovski A, Engelbrecht J and Maugin GA, 2008. Numerical simulation of waves and fronts in inhomogeneous solids, Series A, Vol. 62, World Scientific, Hackensack, NJ.

Berthelot J-M and Le Corre J-F, 2000. Statistical analysis of the progression of transverse cracking and delamination in cross-ply laminates, Composites Science and Technology, 60: 2659-2669.

Berthelot J-M, 2003. Transverse cracking and delamination in cross-ply glass-fiber and carbonfiber reinforced plastic laminates: Static and fatigue loading, Applied Mechanics Reviews, 56(1): 111-147.

Berthelot J-M, Le Corre J-F, 1999. Modelling the transverse cracking in cross-ply laminates: application to fatigue, Composite Part B: Engineering, 30: 569-577.

Berthelot J-M, Leblond P, El Mahi A and Le Core J-F, 1996. Transverse cracking of cross-ply laminates: Part 1. analysis, Composites Part A: Applied Science and Manufacturing, 27(10): 989-1001.

Birman V and Byrd LW, 2007. Modeling and analysis of functionally graded materials and structures. Applied Mechanics Reviews, 60: 195-215.

Bodner SR, Partom Y 1975. Constitutive equations for elastic-viscoplastic strainhardening materials, Journal of Appllied Mechanics, 42(2): 385-389.

Bolotin VV, 1996. Delaminations in composite structures: its origin, buckling, growth and stability, Composites Part B: Engineering, 27(2):129-145.

Bouhala L, Makradi A, Belouettar S, Younes A, Natarajan S, 2015. An XFEM/CZM based inverse method for identification of composite failure parameters, Computers and Structures, 153: 91-97.

Broek D, 1991, Elementary engineering fracture mechanics, 4th revised ed: Kluwer Academic Publishers, 1991.

Buchholz FG, Grebner H, Dreyer KH and Krome H, 1988. 2D- and 3D- applications of the improved and generalized modified crack closure integral method, in Computational Mechanics '88, S. N. Atluri and G. Yagawa, Eds., 1988.

Bufler H, 1971. Theory of elasticity of a multilayered medium, Journal of Elasticity, 1(2): 125143.

Buryachenko VA, 2007. Micromechanics of Heterogeneous Materials. Springer, New York.

Byskov E, 1970. The calculation of stress intensity factors using the finite element method with cracked elements, International Journal of Fracture Mechanics, 6(2): 159-167. 
Camacho GT and Ortiz M, 1996. Computational modeling of impact damage in brittle materials, Journal of the Mechanics and Physics of Solids, 33(20-22): 2899-2938.

Carolan D, Tukovic Z, Murphy N and Ivankovic A, 2013. Arbitrary crack propagation in multiphase materials using the finite volume method, Computational Materials Science, 69: 153-159.

Cavalcante MAA and Pindera M-J, 2012a. Generalized finite-volume theory for elastic stress analysis in solid mechanics. Part I: framework, Journal of Applied Mechanics, 79: 051006(1-11).

Cavalcante MAA and Pindera M-J, 2012b. Generalized finite-volume theory for elastic stress analysis in solid mechanics. Part II: results. Journal of Applied Mechanics, 79: 051007(1-13).

Cavalcante MAA and Pindera MJ, 2014a. Generalized FVDAM theory for periodic materials undergoing finite deformations-Part I: framework, Journal of Applied Mechanics, 81(2): 021005(1-10).

Cavalcante MAA and Pindera MJ, 2014b. Generalized FVDAM theory for periodic materials undergoing finite deformations - part II: results, Journal of Applied Mechanics 81(2): 021006(112).

Cavalcante MAA and Pindera M-J, 2016. Generalized FVDAM theory for elastic-plastic periodic materials, International Journal of Plasticity, 77: 90-117.

Cavalcante MAA, 2006. Modelling of the transient thermo-mechanical behavior of composite material structures by the finite-volume theory, MS Thesis, Federal University of Alagoas, Maceio, Alagoas, Brazil.

Cavalcante MAA, Khatam H and Pindera M-J, 2011. Homogenization of elastic-plastic periodic materials by FVDAM and FE approaches - An assessement, Composites Part B: Engineering, 42: 1713-1730.

Cavalcante MAA, Marques SPC and Pindera M-J, 2007a. Parametric formulation of the finitevolume theory for functionally graded materials. Part I: analysis, Journal of Applied Mechanics, 74 (5): 935-945.

Cavalcante MAA, Marques SPC and Pindera M-J, 2007b. Parametric formulation of the finitevolume theory for functionally graded materials. Part II: Numerical Results, Journal of Applied Mechanics, 74(5): 946-957.

Cavalcante MAA, Marques SPC and Pindera M-J, 2008. Computational aspects of the parametric finite-volume theory for functionally graded materials, Journal of Computational Materials Science, 44 (2): 422-438.

Cavalcante MAA, Pindera M-J and Khatam H, 2012. Finite-volume micromechanics of periodic materials: Past, Present and Future, Composites Part B: Engineering, 43(6): 2521-2543. 
Cavalcantea MAA, Marques SPC, 2014. Homogenization of periodic materials with viscoelastic phases using the generalized FVDAM, Computational Materials Science, 87:43-53.

Center for Nondestructive Evaluation, Iowa State University, https://www.cnde.iastate.edu/research/ultrasonic/ultrasonic-and-composites/modeling-cracksand-delaminations-in-carbon-fiber-composites/

Chan SK, Tuba IS and Wilson WK, 1970. On the finite element method in linear fracture mechanics, Engineering Fracture Mechanics, 2(1): 1-17.

Chandra N, Li H, Shet C and Ghonem H, 2002. Some issues in the application of cohesive zone models for metal-ceramic interfaces, International Journal of Solids and Structures, 39(10): 2827-2855.

Charalambakis N and Murat F, 2006. Homogenization of stratified thermoviscoplastic materials, Quarterly of Applied Mathematics, 64(2): 359-399.

Charalambakis N, 2010. Homogenization techniques and micromechanics. A survey and perspectives, Applied Mechanics Reviews, 63(3): 030803(1-10).

Chatterjee SN, 1979. On interlaminar defects in laminated composites, modern developments in composite materials and structures (Vinson, J. R. Ed.), ASME, New York, 1-15.

Chatterjee SN, 1987. Three- and two-dimensional stress fields near delaminations in laminated composite plates, International Journal of Solids and Structures, 23(11): 1535-1549.

Chatterjee SN, Pindera M-J, Pipes RB. and Dick B, 1982. Composite defect significance, MSC TFR 1312/1108 (NADC Report No.81034-60), Spring house, PA.

Chatzigeorgiou G, Charalambakis N, and Murat F, 2008. Homogenization problems of a hollow cylinder made of elastic materials with discontinuous properties, International Journal of Solids and Structures, 45 (18-19): 5165-5180.

Chen FHK and Shield RT, 1977. Conservation laws in elasticity of the J-Integral type, Zeitschrift für angewandte Mathematik und Physik ZAMP, 28(1): 1-22.

Chen J, Peng W, Ge R, Wei J, 2009. Optimal design of composite laminates for minimizing delamination stresses by particle swarm optimization combined with FEM, Structural Engineering and Mechanics, 31(4): 407-421.

Chen L and Pindera M-J, 2007a. Plane analysis of finite multilayered media with multiple aligned cracks-Part I: theory, Journal of Applied Mechanics, 74(1): 128-143.

Chen L and Pindera M-J, 2007b. Plane analysis of finite multilayered media with multiple aligned cracks-Part II: numerical results, Journal of Applied Mechanics, 74(1): 144-160. 
Chen Q, Chen X, Zhai Z, Yang Z, 2016, A new and general formulation of three-dimensional finite-volume micromechanics for particulate reinforced composites with viscoplastic phases, Composites Part B: Engineering, 85:216-232.

Choi HJ and Thangjitham S, 1994. The interlaminar crack-tip response in a fiber-reinforced composite laminate, International Journal of Fracture, 66(2): 121-138.

Crossman FW and Wang ASD, 1982. The dependence of transverse cracks and delamination on ply thickness in graphite-epoxy laminates, In Damage in Composite Materials. STP 775, American Society for Testing and Materials, Philadelphia, pp. 118-139.

Dattaguru B, Venkatesha KS, Ramamurthy TS, Buchholz FG, 1994. Finite element estimates of strain energy release rate components at the tip of an interface crack under mode I loading, Engineering Fracture Mechanics, 49: 451-463.

Delameter WR, Herrmann G and Barnett DM, 1975. Weakening of an elastic solid by a rectangular array of cracks, Journal of Applied Mechanics, 42(1), 74-80; Errata: 1977, Journal of Applied Mechanics, 44(1): 190.

Demirdzic I and Martinovic D, 1993. Finite volume method for thermo-elastic-plastic stress analysis, Computer Methods in Applied Mechanics and Engineering, 109: 331-349.

Demirdzic I and Muzaferija S, 1994. Finite volume method for stress analysis in complex domains, International Journal for Numerical Methods in Engineering, 37: 3751-3766.

Demirdzic I, Martinovic D and Ivankovic A, 1988. Numerical simulation of thermomechanical deformation processes in a welded work-piece. Zavarivanje, 31: 209-219 (in Serbo-Croat).

Dharani LR, Wei J, Ji FS and Zhao JH, 2003. Saturation of transverse cracking with delamination in polymer cross-ply composite laminates, International Journal of Damage Mechanics, 12: 89-114.

Dieter GE, 1976, Mechanical metallurgy, 2nd ed., McGraw-Hill, New York, pp. 451-489.

Drago AS and Pindera M-J, 2008. A locally exact homogenization theory for periodic microstructures with isotropic phases, Journal of Applied Mechanics, 75(5): 051010(1-14).

Duarte CAM and Oden JT, 1996. H-p clouds - an h-p meshless method. Numerical Methods for Partial Differential Equations, 12: 673-705.

Dugdale DS, 1960. Yielding of steel sheets containing slits, International Journal of Solids and Structures, 8(2):100-104. 
Elguedj T, Gravouil A, Combescure A, 2006. Appropriate extended functions for X-FEM simulation of plastic fracture mechanics, Computer Methods in Applied Mechanics and Engineering, 195:501-515.

Elices M, Guinea GV, Gomerz J and Planas J, 2002, The cohesive zone model: advantages, limitations and challenges, Engineering Fracture Mechanics, 69(2):137-163.

Erdogan F and Gupta GD, 1971. The stress analysis of multi-layered composites with a flaw, International Journal of Solids and Structures, 7(1): 39-61.

Erdogan F, 1971. Layered composites with an interface Flaw, International Journal of Solids and Structures, 7(8): 1089-1107.

Eshelby JD, 1957. The determination of the elastic field of an ellipsoidal inclusion and related problems, Proceedings of the Royal Society A: 241(1226): 376-396.

Fallah N, 2006. On the use of shape functions in the cell centered finite volume formulation for plate bending analysis based on Mindlin-Reissner plate theory, Computers and Structures, 84: 1664-1672.

Fallah N, 2008. A method for the calculation of face gradients in the two-dimensional, cell centred, finite volume formulation for stress analysis in solid problems, Scientia Iranica (International Journal of Science and Technology), 15(3): 286-294.

Fallah N, Bailey C, Cross M and Taylor GA, 2000. Comparison of finite element and finite volume methods application in geometrically nonlinear stress analysis, Applied Mathematical Modelling, 24: 439-455.

Freed Y and Banks-Sills L, 2005. A through interface crack between a $\pm 45^{\circ}$ transversely isotropic pair of materials, International Journal of Fracture, 133: 1-41.

Fryer YD, Bailey C, Cross M and Lai C-H, 1991. A control volume procedure for solving the elastic stress-strain equations on an unstructured mesh, Applied Mathematical Modelling, 15: 639-645.

Garg AC, 1988. Delamination - a damage mode in composite structures, Engineering Fracture Mechanics, 29(5): 557-584.

Garrett KW and Bailey JE, 1977. Multiple transverse fracture in $90^{\circ}$ cross-ply laminates of a glass fibre-reinforced polyester, Journal of Materials Science, 12: 157-168.

Gattu M, Khatam H, Drago AS and Pindera M-J, 2008. Parametric finite-volume micromechanics of uniaxial, continuously-reinforced periodic materials with elastic phases, Journal of Engineering Materials and Technology, 130 (3): 031015(1-15). 
Geubelle PH and Baylor JS, 1998. Impact-induced delamination of composites: a 2D simulation, Composites Part B: Engineering, 29(5): 589-602.

Griffith AA, 1921. The phenomena of rupture and flow in solids, Philosophical Transactions of the Royal Society of London, Series A 221: 163-198.

Griffith AA, 1924. The theory of rupture, In Proceedings of the First International Congress of Applied Mechanics (Biezeno and Burgers, Eds.), Waltman, 55-63.

Groves SE, Harris CE, Highsmith AL, Allen DH and Norvell RG, 1987. An experimental and analytical treatment of matrix cracking in cross-ply laminates, Experimental Mechanics, 22: 7379.

Gudmundson P and Ostlund S, 1992. Prediction of thermoelastic properties of composite laminates with matrix cracks, Composites Science and Technology, 44: 95-105.

Hashin Z, 1985. Analysis of cracked laminates: a variational approach, Mechanics of Materials, 4: 121-136.

Hashin Z, 1986. Analysis of stiffness reduction of cracked cross-ply laminates, Engineering Fracture Mechanics, 25: 771-778.

Hassan NM and Batra RC, 2008. Modeling damage in polymeric composites, composites Part B: Engineering, 39(1), 66-82.

Henshell RD and Shaw KG, 1975. Crack tip finite elements are unnecessary, International Journal for Numerical Methods in Engineering, 9(3): 495-507.

Herakovich CT, 1998, Mechanics of fibrous composites, John Wiley \& Sons, Inc., New York.

Highsmith AL and Reifsnider KL, 1982. Stiffness-reduction mechanisms in composite laminates, ASTM STP 775, K.L. Reifsnider, Ed., Americal Society for Testing and Materials. pp. 103-117.

Hill R, 1963. Elastic properties of reinforced solids: some theoretical principles, Journal of the Mechanics and Physics of Solids, 11(5): 357-372.

Hoiseth K, Qu J, 2003. Cracking paths at the ply interface in a cross-ply laminate, Composite Part B: Engineering, 34: 437-445.

Huang ZQ, Nie GH, Chan CK, 2011. An exact solution for stresses in cracked composite laminates and evaluation of the characteristic damage state, Composites Part B: Engineering, 42(5): 1008-1014.

Hwu C and Hu JS, 1992. Stress intensity factors and energy release rates of delaminations in composite laminates, Engineering Fracture Mechanics, 42: 97-988. 
Irwin GR, 1958. Fracture, Handbud der Physik, 6, 551.

Isida M, 1971. Effect of width and length on stress intensity factors of internally cracked plates under various boundary conditions, International Journal of Fracture Mechanics, 7(3): 301-316.

Joffe R and Varna J, 1999. Analytical modeling of stiffness reduction in symmetric and balanced laminates due to cracks in 90 layers, Composites Science and Technology, 59: 1641-1652.

Johnson WS, Lubowinski SJ and Highsmith AL, 1990. Mechanical characterization of unnotched SCS6/Ti-15-3 metal matrix composites at room temperature, Thermal and Mechanical Behavior of Metal Matrix and Ceramic Matrix Composites, J. M. Kennedy, H. H. Moeller, W. S. Johnson, eds., American Society for Testing and Materials, Philadelphia, PA, pp. 193-218, Paper No. ASTM STP 1080.

Kennedy J and Eberhart R, 1995. Particle swarm optimization. In: Proceedings of IEEE International Conference on Neural Networks, 1942-1948.

Khatam H and Pindera M-J, 2009a. Thermo-elastic moduli of lamellar composites with wavy architectures, Composites Part B: Engineering, 40 (1): 50-64.

Khatam H and Pindera M-J, 2009b. Parametric finite-volume micromechanics of periodic materials with elastoplastic phases, International Journal of Plasticity, 25(7): 1386-1411.

Khatam H and Pindera M-J, 2012. Microstructural scale effects in the nonlinear elastic response of bio-inspired wavy multilayers undergoing finite deformation, Composites Part B: Engineering, 43(3): 869-884.

Koiter WT, 1959. An infinite row of collinear cracks in an infinite elastic sheet, IngenieurArchiv, 28: 168-173.

Krueger R, 1994. Three dimensional finite element analysis of multidirectional composite DCB, SLB and ENF specimens, Institute for Statics and Dynamics of Aerospace Structures, University of Stuttgart ISDReport No. 94/2.

Krueger R, 2002. The virtual crack closure technique: history, approach and applications, NASA/CR-2002-211628, ICASE Report No. 2002-10.

Kulkarni M, 2012. Finite element analysis of 2-D representative volume element, Master Thesis, Michigan Technology University.

Lee JH and Hong CS, 1993. Refined two-dimensional analysis of cross-ply laminates with transverse cracks based on the assumed crack opening deformation. Composites Science and Technology, 46: 157-166. 
Leveque RJ, 2002. Finite volume methods for hyperbolic problems. Cambridge University Press, Cambridge, UK.

Li FZ,Shih CF and Needleman A, 1985. A comparison of methods for calculating energy release rates, Engineering Fracture Mechanics, 21(2): 405-421.

Liao J, Vesely I, 2003a. A structural basis for the size-related mechanical properties of mitral valve chordae tendineae, Journal of Biomechanics, 36(8): 1125-1133.

Liao J, Vesely I, 2003b. Mechanical and structural properties of mitral valve chordae tendineae. Heart Valve Laboratory, BME, Cleveland Clinic Foundation. PPT Presentation Communicated to Engineered Materials Concepts, LLC in fulfillment of the Subcontract Requirements through the DHRP Grant No. DAMD17-01-1-0673.

Lowengrub M, 1966. A two-dimensional crack problem, International Journal of Engineering Science, 4(3): 289-299.

Lu and Hutchinson, 1995. Effect of matrix cracking and interface sliding on the thermal expansion of fibre-reinforced composites, Composites, 26(6): 403-414.

Martin RH,1998. Incorporating interlaminar fracture mechanics into design, in International Conference on Designing Cost-Effective Composites: IMechE Conference Transactions, London, U.K., pp. 83-92.

Matous K and Geubelle P H, 2006. Multiscale modelling of particle debonding in reinforced elastomers subjected to finite deformations, International Journal for Numerical Methods in Engineering, 65(2): 190-223.

McCartney LN, 2013. Energy methods for modelling damage in laminates, Journal of Composite Materials, 47(20-21): 2613-2640.

Melenk JM and Babuska I, 1996. The partition of unity finite element method: basic theory and applications, Computer Methods in Applied Mechanics and Engineering, 39:289-314.

Melenk JM, 1995. On generalized finite element methods, PhD Thesis, University of Maryland.

Moes N, Dolbow J and Belytschko T, 1999. A finite element method for crack growth without remeshing, International Journal for Numerical Methods in Engineering, 46:131-50.

Muskhelishvili NI, 1953a. Some basic problems of the mathematical theory of elasticity, P. Noordhoff, Groningen, The Netherlands.

Muskhelishvili, NI, 1953b. Singular integral equations, P. Noordhoff, Groningen, The Netherlands. 
Narayana KB and Dattaguru B, 1996. Certain aspects related to computation by modified crack closure integral (MCCI), Engineering Fracture Mechanics, 55: 335-339.

Narayana KB, Dattaguru B, Ramamurthy TS and Vijayakumar K, 1990. Modified crack closure integral using six-noded isoparametric quadrilateral singular elements, Engineering Fracture Mechanics, 36: 945-955.

Needleman A 1987. A continuum model for void nucleation by inclusion debonding, Journal of Applied Mechanics, 54(3):525-531.

Njuhovic E, Brau M, Wolff-Fabris F, Starzynski K and Altstadt V, 2015. Identification of failure mechanisms of metallised glass fibre reinforced composites under tensile loading using acoustic emission analysis, Composites Part B: Engineering, 81, 1-13.

O'Brien TK, 1982. Characterization of delamination onset and growth in a composite laminate, In: Damage in Composite Materials, ASTM STP 775, pp. 140-167.

O'Brien TK, 1998. Interlaminar fracture toughness: The long and winding road to standardization, Composites Part B: Engineering, 29: 57-62.

Ortiz M and Suresh S, 1993. Statistical properties of residual stresses and intergranular fracture in ceramic materials, Journal of Applied Mechanics, 60(1):77-84.

Pagano NJ and Schoeppner GA, 2000. Delamination of polymer matrix composites: problems and assessment. In: Comprehensive Composite Materials, Vol. 2, A. Kelly and C. Zweben, Eds.: Elsevier Science Ltd., pp. 433-528.

Paley M and Aboudi J, 1992. Micromechanical analysis of composites by the generalized method of cells, Mechanics of Materials, 14: 127-139.

Pan W, Wheel MA and Qin Y, 2010. Six-node triangle finite volume method for solids with a rotational degree of freedom for incompressible material, Computers \& Structures, 88: 15061511.

Papadopoulos DS and Bowles KJ, 1990. Use of unbalanced laminates as a screening method for microcracking, NASA Technical Memorandum 102517.

Paris F, Blazquez A, McCartney LN and Barroso A, 2010b. Characterization and evolution of matrix and interface related damage in [0/90]s laminates under tension. Part II: experimental evidence, Composite Science Technology, 70: 1176-1183.

París F, Blazquez A, McCartney LN and Mantic V, 2010a. Characterization and evolution of matrix and interface related damage in [0/90]s laminates under tension. Part I: numerical predictions, Composite Science Technology, 70: 168-1175. 
Park K, and Paulino GH, 2011. Cohesive zone models: A critical review of traction-separation relationships across fracture surfaces, Applied Mechanics Reviews, 64(6): 060802(1-20).

Parks DM, 1974. A stiffness derivative finite element technique for detennination of crack tip stress intensity factors, International Journal of Fracture, 10(4): 487-502.

Parvizi A and Bailey JE, 1978. On multiple transverse cracking in glass fiber epoxy cross-ply laminates, Journal of Materials Science, 13: 2131-2136.

Paulino GH, Pindera M-J, Dodds RH, Rochinha FE, Dave EV and Chen L (Ed.), 2008. Multiscale and Functionally Graded Materials. AIP Conference Proceedings 973. Melville, New York.

Peng W, Chen J, Wei J and Tu W, 2011. Optimal strength design for fiber-metal laminates and fiber-reinforced plastic laminates, Journal of Composite Materials, 45(2): 237-254.

Pindera M-J, 1981. An endochronic theory for transversely isotropic fibrous composites, Ph.D. thesis, Blacksburg, Virginia.

Pindera M-J, 1986. Mechanics of composite materials: ESM 4040 Lecture Notes. Blacksburg, VA: Virginia Tech.

Pindera M-J, 1991. Local/global stiffness matrix formulation for composite materials and structures, Composites Engineering, 1(2): 69-83.

Pindera M-J, 1992. Discussion of the paper: stress analysis of multilayered anisotropic elastic media by Choi and Thangjitham, Journal of Applied Mechanics, 59: 238-239.

Pindera M-J, Khatam H, Drago AS and Bansal Y, 2009. Micromechanics of spatially uniform heterogeneous media: a critical review and emerging approaches, Composites Part B: Engineering, 40 (5): 349-378.

Que NS and Tin-Loi F, 2002. An optimization approach for indirect identification of cohesive crack properties, Computers and Structures, 80: 1383-1392

Raghavan P and Ghosh S, 2005. A continuum damage mechanics model for unidirectional composites undergoing interfacial debonding, Mechanics of Materials, 37(9): 955-979.

Raju IS, 1987. Calculation Of strain-energy release rates with higher order and singular finite elements, Engineering Fracture Mechanics, 28: 251-274.

Raju IS, Hivakumar KN and Rews JH, 1988. Three-dimensional elastic analysis of a composite double cantilever beam specimen, AIAA Journal, 26(12): 1493-1498. 
Ramamurthy TS, Krishnamurthy T, Badari Narayana K, Vijayakumar K and Dattaguru B, 1986. Modified crack closure integral method with quarter point elements, Mechanics Research Communications, 13: 179-186.

Ran Z, Yan Y, Li J, Qi Z and Yang L, 2014. Determination of thermal expansion coefficients for unidirectional fiber-reinforced composites, Chinese Journal of Aeronautics, 27(5): 1180-1187.

Remmers JJC, de Borst R, and Needleman A, 2008. The simulation of dynamic crack propagation using the cohesive segments method, Journal of the Mechanics and Physics of Solids, 56: 70-92.

Rice JR and Sih GC, 1965. Plane problems of cracks in dissimilar media, Journal of Applied Mechanics, 32(2): 418-423.

Rice JR, 1968. A path independent integral and the approximate analysis of strain concentrations by notches and cracks, Journal of Applied Mechanics, 35(2): 379-386.

Rybicki EF and Kanninen MF, 1977. A finite element calculation of stress intensity factor by a modified crack closure integral, Engineering Fracture mechanics, 9: 931-938.

Schoeppner GA and Pagano NJ, 1999. 3-D thermoelastic moduli and saturation crack density for cross-ply laminates with transverse cracks. International Journal of Damage Mechanics, 8: 273309.

Sethuraman R and Maiti SK, 1988. Finite element based computation of strain energy release rate by modified crack closure integral, Engineering Fracture Mechanics, 30: 227-231.

Shi Y, Pinna C and Soutis C, 2014. Interface cohesive elements to model matrix crack evolution in composite laminates, Applied Composite Materials, 21: 57-70.

Smith PA and Wood JR, 1990. Poisson's ratio as a damage parameter in the static tensile loading of simple cross-ply laminates, Composite Science Technology, 38: 85-93.

Sneddon IN and Lowengrub M, 1969. Crack problems in the classical theory of elasticity, John Wiley, New York.

Sneddon IN and Srivastava KN, 1965. The stress in the vicinity of an infinite row of collinear cracks in an elastic body, Proceedings of the Royal Society of Edinburgh, A67, 39-49.

Sneddon IN, 1951. Fourier Transforms, McGraw-Hill, New York.

Sneddon IN, 1961. Fourier Series, Routledge and Paul, London.

Soden PD, Hinton MJ and Kaddour AS, 1998. Lamina properties, lay-up configurations and loading conditions for a range of fiber-reinforced composite laminates, Composites Science and Technology, 58(7): 1011-1022. 
Song J-H, Areias PMA and Belytschko T, 2006. A method for dynamic crack and shear band propagation with phantom nodes, International Journal for Numerical Methods in Engineering, 67: 868-893.

Song SH, Paulino GH and Buttlar WG, 2006. A bilinear cohesive zone model tailored for fracture of asphalt concrete considering viscoelastic bulk material, Engineering Fracture Mechanics, 73(18): 2829-2848.

Stylianou V and Ivankovic A, 2002. Finite volume analysis of dynamic fracture phenomena, part II: a cohesive zone type modelling, International Journal of Fracture, 113(2): 107-123.

Sukumar N and Prevost JH, 2003. Modeling quasi-static crack growth with the extended finite element method Part I: computer implementation, International Journal of Solids and Structures, 40: 7513-7537.

Sukumar N, Huang ZY, Prevost JH and Suo Z, 2004. Partition of unity enrichment for bimaterial interface cracks, International Journal for Numerical Methods in Engineering, 59: 1075-1102.

Sun CT and Qian W, 1997. The use of finite extension strain energy release rates in fracture of interfacial cracks, International Journal of Solids and Structures, 34: 2595-2609.

Sun CT and Jih CJ, 1987. On strain energy release rates for interfacial cracks in bi-material media, Engineering Fracture Mechanics, 28: 13-20.

Sun CT and Manoharan MG, 1989. Strain energy release rates of an interfacial crack between two orthotropic solids, Journal of Composite Materials, 23: 460-478.

Suquet PM, 1987. Elements of homogenization for inelastic solid mechanics. Lecture Notes in Physics, 272, 193-278. Springer-Verlag, Berlin (New York).

Svensson D, Alfredsson KS, Biel A, Stigh U, 2014. Measurement of cohesive laws for interlaminar failure of CFRP, Composites Science and Technology, 100: 53-62.

Talreja R, 1985. Transverse cracking and stiffness reduction in composite laminates, Journal of Composite Material, 19(4): 355-375.

Tang Z, Wang C and Yu Y, 2015. Failure response of fiber-epoxy unidirectional laminate under transverse tensile/compressive loading using finite-volume micromechanics, Composite Part B: Engineering, 79: 331-341.

Taylor GA, Bailey C and Cross M, 2003. A vertex-based finite volume method applied to nonlinear material problems in computational solid mechanics, International Journal for Numerical Methods in Engineering, 56: 507-529. 
Taylor GA, Bailey C and Cross M, 1995. Solutions of the elastic/visco-plastic constitutive equations: A finite volume approach, Applied Mathematics Modelling, 19: 746-760.

Tracey DM, 1971. Finite elements for determination of crack tip elastic stress intensity factors, Engineering Fracture Mechanics, 3(3), 255-265.

Tu W and Pindera M-J, 2013. Targeting the finite-deformation response of wavy biological tissues with bio-inspired material architectures, Journal of the Mechanical Behavior of Biomedical Materials, 28: 291-308.

Tu W and Pindera M-J, 2014, Cohesive zone-based damage evolution in periodic materials via finite-volume homogenization, Journal of Applied Mechanics, 81(10): 101005(1-16).

Tu W, Tang Z and Pindera MJ, 2012. Interfacial damage mechanics of composite materials via finite-volume micromechanics, 20th Annual International Conference on Composite Materials, July 22-28, Beijing, CHINA.

Turon A, Davila CG, Camanho PP and Costa J, 2007. An engineering solution for mesh size effects in the simulation of delamination using cohesive zone models, Engineering Fracture Mechanics, 74: 1665-1682.

Valoroso N, Sessa S, Lepore M, Cricri G, 2013. Identification of mode-I cohesive parameters for bonded interfaces based on DCB test, Engineering Fracture Mechanics, 104: 56-79.

Vasilev VV, Dudchenko AA and Elpatevskii AN, 1970. Analysis of the tensile deformation of glass-reinforced plastics. Mekhanika Polimerov, 1: 144-147.

Versteeg HK and Malalasekera W, 2007. An introduction to computational fluid dynamics: The finite volume method. Prentice-Hall, New York.

Walsh PF, 1971. The computation of stress intensity factors by a special finite element technique, International Journal of Solids and Structures, 7(10): 1333-1342.

Wang ASD, 1984. Fracture mechanics of sublaminate cracks in composite materials, Composites Technology Review, 6(2): 45-62.

Wang ASD, Kishore NN and Li CA, 1985. Crack development in graphite-epoxy cross-ply laminates under uniaxial tension, Composites Science and Technology, 24(1): 1-31.

Wang BL and Han JC, 2010. Transverse cracking of orthotropic composite laminates: a fracture mechanics approach, Archive of Applied Mechanics, 80: 1301-1316.

Wang G and Pindera M-J, 2016. Locally-exact homogenization of unidirectional composites with coated or hollow reinforcement, Materials \& Design, 93: 514-528. 
Wenke $\mathrm{P}$ and Wheel MA, 2003. A finite volume method for solid mechanics incorporating rotational degrees of freedom, Computers and Structures, 81: 321-329.

Westergaard HM, 1939. Bearing pressures and cracks, Journal of Applied Mechanics, Series A, 66, A49-A53.

Wheel MA, 1996. A finite-volume approach to the stress analysis of pressurized axisymmetric structures, International Journal of Pressure Vessels and Piping, 68: 311-317.

Wheel MA, 1999. A mixed finite volume formulation for determining the small strain deformation of incompressible materials, International Journal for Numerical Methods in Engineering, 44: 1843-1861.

Wheel MA, 2008. A control volume-based finite element method for plane micropolar elasticity, International Journal for Numerical Methods in Engineering, 75: 992-1006.

Wilkins DJ, Eisenmann JR, Camin RA, Margolis WS, Benson RA, 1982. Characterizing delamination growth in graphite-epoxy. In: Damage in composite materials, ASTM STP 775, K. L. Reifsnider, Ed., American Society for Testing and Materials, 168-183.

Xu XP and Needleman A, 1994. Numerical simulation of fast crack growth in brittle solids, Journal of the Mechanics and Physics of Solids, 42(9): 1397-1434.

Yau JF, Wang SS and Corten HT, 1980. A mixed-mode crack analysis of isotropic solids using conservation laws of elasticity, Journal of Applied Mechanics, 47(2): 335-341.

Zhong Y, Bansal Y and Pindera M-J, 2004. Efficient reformulation of the thermal higher-order theory for FGM's with variable thermal conductivity, International Journal of Computational Science and Engineering, 5 (4): 795-831. See also: NASA CR 2002-211910, November 2002.

Zienkiewicz OC, Taylor RL., 2000. The Finite element method, vol. 1-3. ButterworthHeinemann: Oxford. 


\section{Appendix I:}

Vasilev et al. (1970) considered the axial deformation of a symmetric cross-ply laminate with glass reinforcement loaded by an axial force $P$ in the $\mathrm{x}-\mathrm{z}$ plane, with the inner $90^{\circ}$ ply thickness $2 h_{2}$, outer $0^{0}$ ply thickness $h_{1}$, and unit width. Assuming that the axial stress $\sigma_{x x}$ in both plies does not vary with the $\mathrm{z}$ coordinate in the presence of a single vertical crack in the inner $90^{\circ}$ plies caused by the applied force $\mathrm{p}=\mathrm{p}_{1}$, the authors derived the following solution for the variation of the axial and shear stress in the 90 plies measured from the crack face,

$$
\begin{gathered}
\left.\sigma_{x x}(x)\right|_{90^{o}}=\sigma_{x x}^{o}\left[1-e^{-k_{1} x}\left(\frac{k_{1}}{k_{2}} \sin _{2} x+\cos k_{2} x\right)\right] \\
\left.\sigma_{x z}(x, z)\right|_{90^{o}}=\sigma_{x x}^{o} z\left(\frac{k_{1}^{2}+k_{2}^{2}}{k_{2}}\right) e^{-k_{1} x} \sin _{2} x
\end{gathered}
$$

where $k_{1,2}=\sqrt{1 / 2\left(b^{2} \pm a^{2}\right)}$ and

$$
\begin{gathered}
a^{2}=\frac{\frac{1}{3} \frac{h_{2}^{3}}{G_{2}}+\frac{1}{3 G_{1}} h_{1} h_{2}^{2}-\frac{v_{3}}{E_{2}}\left(\frac{2}{3} h_{2}^{3}+h_{1} h_{2}^{2}\right)+\frac{1}{6}\left(\frac{v_{1}}{E_{1}}+\frac{v_{2}}{E_{2}}\right) h_{1} h_{2}^{2}}{\frac{1}{2 E_{2}}\left[\frac{1}{5} h_{2}^{5}-\frac{2}{3} h_{2}^{4}\left(h_{1}+h_{2}\right)+h_{2}^{3}\left(h_{1}+h_{2}\right)^{2}+\frac{1}{5} h_{1}^{3} h_{2}^{2}\right]} \\
b^{4}=\frac{2 h_{2}^{2}\left(\frac{1}{h_{1} E_{1}}+\frac{1}{h_{2} E_{2}}\right)}{\frac{1}{2 E_{2}}\left[\frac{1}{5} h_{2}^{5}-\frac{2}{3} h_{2}^{4}\left(h_{1}+h_{2}\right)+h_{2}^{3}\left(h_{1}+h_{2}\right)^{2}+\frac{1}{5} h_{1}^{3} h_{2}^{2}\right]}
\end{gathered}
$$

The maximum value of $\left.\sigma_{x x}(x)\right|_{90^{\circ}}$ occurs at $x=\pi / k_{2}$ where $\left.\sigma_{x z}(x, z)\right|_{90^{o}}$ is 0 . At this point, $\left.\sigma_{x x}(x)\right|_{90^{\circ}}$ is greater than the transverse ply strength and hence it may be assumed that a system of vertical cracks spaced $\pi / k_{2}$ apart forms in the $90^{\circ}$ plies.

For $\mathrm{p}>\mathrm{p}_{1}$ the axial and shear stresses in the $90^{\circ}$ plies measured from the origin centered between two adjacent cracks are,

$$
\begin{gathered}
\left.\sigma_{x x}(x)\right|_{90^{o}}=\frac{\sigma_{x x}^{o}}{\operatorname{sh}\left(\frac{\pi k_{1}}{2 k_{2}}\right)}\left[\operatorname{sh}\left(\frac{\pi k_{1}}{2 k_{2}}\right)-\frac{k_{1}}{k_{2}} \operatorname{ch}\left(k_{1} x\right) \cos k_{2} x-\operatorname{sh}\left(k_{1} x\right) \sin k_{2} x\right] \\
\left.\sigma_{x z}(x, z)\right|_{0^{o}}=\sigma_{x x}^{o} z\left(\frac{k_{1}^{2}+k_{2}^{2}}{k_{2}}\right) \frac{\operatorname{sh}\left(k_{1} x\right) \cos k_{2} x}{\operatorname{sh}\left(\frac{\pi k_{1}}{2 k_{2}}\right)}
\end{gathered}
$$


with the maximum occurring at $\mathrm{x}=0$. A new set of cracks will form at $\mathrm{p}=\mathrm{p}_{2}$ for which $\left.\sigma_{x x}(x)\right|_{90^{\circ}}$ attains the transverse ply strength. This progressive fracture process of the $90^{\circ}$ plies will continue until the outer $0^{o}$ plies fail. 